
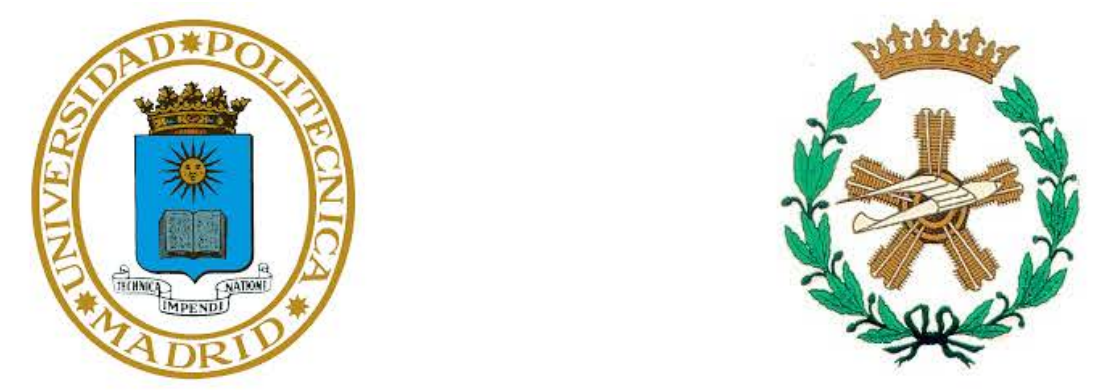

Universidad Politécnica de MAdRid Escuela TÉCnica Superior de IngEniERos AERONÁuticos

\title{
ANÁLISIS PARAMÉTRICO \\ DETERMINISTA Y ALEATORIO DE PROBLEMAS DINÁMICOS EN ESTRUCTURAS AEROESPACIALES
}

Tesis Doctoral:

\section{Iñaki Armendáriz Benítez}

Ingeniero Aeronáutico

Licenciado en Ciencias Matemáticas

MadRID Noviembre 2015 


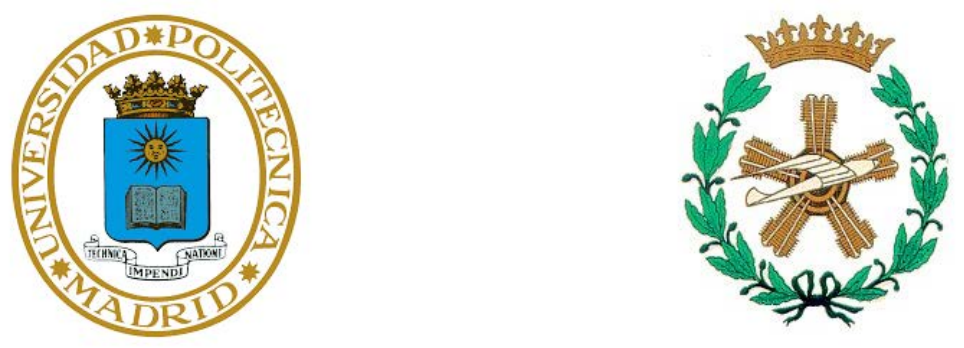

Departamento de Matemática Aplicada y Estadística

Escuela Técnica Superior de Ingenieros Aeronáuticos

Universidad Politécnica de Madrid

\section{Análisis paramétrico determinista y aleatorio de problemas dinámicos en estructuras aeroespaciales}

Iñaki Armendáriz Benítez

Ingeniero Aeronáutico

Licenciado en Ciencias Matemáticas

Director de la Tesis Doctoral:

José Olarrea Busto

Doctor en Ciencias Físicas

Madrid Noviembre 2015 



\title{
TRIBUNAL
}

\author{
Presidente: \\ Ignacio Esteban Parra Fabián \\ Doctor Ingeniero Aeronáutico \\ Catedrático de Universidad \\ Universidad Politécnica de Madrid
}

\begin{abstract}
Vocal:
Ricardo Atienza Pascual

Doctor Ingeniero Aeronáutico

Universidad Alfonso X el Sabio
\end{abstract}

Vocal:

Fernando Cabrerizo García

Doctor Ingeniero Aeronáutico

Universidad Alfonso X el Sabio

Suplente:

Pablo Fajardo

Doctor Ingeniero Aeronáutico

Universidad Carlos III de Madrid

\author{
Vocal: \\ Malte Frövel \\ Doctor en Ingeniería \\ Instituto Nacional de Técnica \\ Aeroespacial \\ Secretario: \\ Marcos Chimeno Manguán \\ Doctor Ingeniero Aeronáutico \\ Universidad Politécnica de Madrid
}

Suplente:

José María Pintado

Sanjuanbenito

Doctor Ingeniero Aeronáutico

Instituto Nacional de Técnica

Aeroespacial 



\section{Resumen}

Los fenómenos dinámicos pueden poner en peligro la integridad de estructuras aeroespaciales y los ingenieros han desarrollado diferentes estrategias para analizarlos. Uno de los grandes problemas que se plantean en la ingeniería es cómo atacar un problema dinámico estructural. En la presente tesis se plantean distintos fenómenos dinámicos y se proponen métodos para estimar o simular sus comportamientos mediante un análisis paramétrico determinista y aleatorio del problema. Se han propuesto desde problemas sencillos con pocos grados de libertad que sirven para analizar las diferentes estrategias y herramientas a utilizar, hasta fenómenos muy dinámicos que contienen comportamientos no lineales, daños y fallos.

Los primeros ejemplos de investigación planteados cubren una amplia gama de los fenómenos dinámicos, como el análisis de vibraciones de elementos másicos, incluyendo impactos y contactos, y el análisis de una viga con carga armónica aplicada a la que también se le añaden parámetros aleatorios que pueden responder a un desconocimiento o incertidumbre de los mismos. Durante el desarrollo de la tesis se introducen conceptos y se aplican distintos métodos, como el método de elementos finitos (FEM) en el que se analiza su resolución tanto por esquemas implícitos como explícitos, y métodos de análisis paramétricos y estadísticos mediante la técnica de Monte Carlo.

Más adelante, una vez ya planteadas las herramientas y estrategias de análisis, se estudian fenómenos más complejos, como el impacto a baja velocidad en materiales compuestos, en el que se busca evaluar la resistencia residual y, por lo tanto, la tolerancia al daño de la estructura. Se trata de un suceso que puede producirse por la caída de herramienta, granizo o restos en la pista de aterrizaje. Otro de los fenómenos analizados también se da en un aeropuerto y se trata de la colisión con un dispositivo frangible, el cual tiene que romperse bajo ciertas cargas y, sin embargo, soportar otras.

Finalmente, se aplica toda la metodología planteada en simular y analizar un posible incidente en vuelo, el fenómeno de la pérdida de pala de un turbohélice. Se trata de un suceso muy particular en el que la estructura tiene que soportar unas cargas complejas y excepcionales con las que la aeronave debe ser capaz de completar con éxito el vuelo. El análisis incluye comportamientos no lineales, daños, y varios tipos de fallos, y en el que se trata de identificar los parámetros clave en la secuencia del fallo. El suceso se analiza mediante análisis estructurales deterministas más habituales y también mediante otras técnicas como el método de Monte Carlo con el que se logran estudiar distintas incertidumbres en los parámetros con variables aleatorias. Se estudian, entre otros, el tamaño de pala perdida, la velocidad y el momento en el que se produce la rotura, y la rigidez y resistencia de los apoyos del motor. Se tiene en cuenta incluso el amortiguamiento estructural del sistema. Las distintas estrategias de análisis permiten obtener unos resultados valiosos e interesantes que han sido objeto de distintas publicaciones. 



\begin{abstract}
Dynamic phenomena can endanger the integrity of aerospace structures and, consequently, engineers have developed different strategies to analyze them. One of the major engineering problems is how to deal with the structural dynamics. In this thesis, different dynamic phenomena are introduced and several methods are proposed to estimate or simulate their behaviors. The analysis is considered through parametric, deterministic and statistical methods. The suggested issues are from simple problems with few degrees of freedom, in order to develop different strategies and tools to solve them, to very dynamic phenomena containing nonlinear behaviors failures, damages.

The first examples cover a wide variety of dynamic phenomena such as vibration analysis of mass elements, including impacts and contacts, and beam analysis with harmonic load applied, in which random parameters are included. These parameters can represent the unawareness or uncertainty of certain variables. During the development of the thesis several concepts are introduced and different methods are applied, such as the finite element method (FEM), which is solved through implicit and explicit schemes, and parametrical and statistical methods using the Monte Carlo analysis technique.

Next, once the tools and strategies of analysis are set out more complex phenomena are studied. This is the case of a low-speed impact in composite materials, the residual strength of the structure is evaluated, and therefore, its damage tolerance. This incident may occur from a tool dropped, hail or debris throw on the runway. At an airport may also occur, and it is also analyzed, a collision between an airplane and a frangible device. The devise must brake under these loads, however, it must withstand others.

Finally, all the considered methodology is applied to simulate and analyze a flight incident, the blade loss phenomenon of a turboprop. In this particular event the structure must support complex and exceptional loads and the aircraft must be able to successfully complete the flight. Nonlinear behavior, damage, and different types of failures are included in the analysis, in which the key parameters in the failure sequence are identified. The incident is analyzed by deterministic structural analysis and also by other techniques such as Monte Carlo method, in which it is possible to include different parametric uncertainties through random variables. Some of the evaluated parameters are, among others, the blade loss size, propeller rotational frequency, speed and angular position where the blade is lost, and the stiffness and strength of the engine mounts. The study does also research on the structural damping of the system. The different strategies of analysis obtain valuable and interesting results that have been already published.
\end{abstract}



Agradecimientos

La primera persona a la que quisiera agradecer su ayuda es a Jesús López Díez con el que inicié esta aventura y con el que tuve el honor de poder compartir las primeras alegrías del esfuerzo invertido. A Marcos Chimeno, Pablo Fajardo y Francisco Herrada que me brindaron su apoyo en los momentos más complicados. A Héctor Climent y Mercedes Oliver con los que tuve el honor de colaborar y contribuyeron al planteamiento de este trabajo. $Y$, por supuesto, a José Olarrea ya que gracias a él hemos podido sacar finalmente esta tesis adelante.

No quisiera tampoco olvidarme de mis compañeros del Área de Estructura y Mecanismos del INTA: Ramón Fernández, Javier San Millán, José Miguel Encinas, Roberto González, Ramón Santiago, Juan García y Rafael Vilanova, que me han facilitado siempre las cosas y con los que he disfrutado enormemente trabajando. Quisiera destacar a Juan García con el que he compartido innumerables proyectos y espero seguir haciéndolo.

Asimismo, agradezco a mi familia y, en especial, a Anabella, mi prometida, la comprensión y la paciencia que han demostrado durante el tiempo que he dedicado a la realización de esta tesis. 



\section{ÍNDICE GENERAL}

Índice general $\quad$ i

Índice de figuras $\quad$ v

Índice de tablas $\quad$ ix

1. Introducción 1

1.1. Presentación del problema . . . . . . . . . . . . . . . . . 1

1.2. Ámbito científico de las herramientas . . . . . . . . . . . . . . . . . . 4

1.2.1. Herramientas para el análisis de vibraciones . . . . . . . . . . . . . . . 7

1.2.2. Métodos de análisis paramétricos . . . . . . . . . . . . . . 8

1.3. Estado de la técnica . . . . . . . . . . . . . . . . . . . . . . . . 11

1.4. Objetivos y alcance de la tesis . . . . . . . . . . . . . . . . . . . 14

1.5. Descripción de la tesis . . . . . . . . . . . . . . . . . . 15

2. Análisis de sistemas dinámicos $\quad 17$

2.1. Tipos de señales . . . . . . . . . . . . . . . . . . . . . . . 17

2.1.1. Señales deterministas _. . . . . . . . . . . . . . . . . 18

2.1.2. Señales aleatorias . . . . . . . . . . . . . . . . . . 18

2.2. Teoría de vibraciones . . . . . . . . . . . . . . . . . . . 20

2.2.1. Tipos de sistemas . . . . . . . . . . . . . . . . . . . . 20

2.2.2. Tipo de vibraciones . . . . . . . . . . . . . . . . . 21

2.3. Métodos de integración . . . . . . . . . . . . . . . . . . . 24

2.3.1. Métodos implícitos y explícitos . . . . . . . . . . . . . . 25

2.3.2. Ejemplo: Sistema de 2 grados de libertad . . . . . . . . . . . . . . 28

2.4. Método de Craig-Bampton . . . . . . . . . . . . . . . . . . . 34

3. Fenómenos dinámicos $\quad 37$

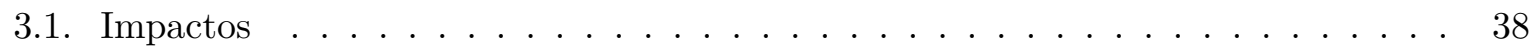

3.1.1. Ejemplo: Choque entre parejas de masas unidas por muelles . . . . . . . . 39 
3.1.2. Ejemplo: Impacto analizado con herramientas FEM avanzadas . . . . . . 40

3.2. Frangibilidad . . . . . . . . . . . . . . . . . . . . 44

3.2.1. Ejemplo: Estructura frangible . . . . . . . . . . . . . . . . . . 44

3.3. Incidentes de vuelo; pérdida de pala de un avión de transporte . . . . . . . . . 50

3.3.1. Análisis del fenómeno de la pérdida de pala . . . . . . . . . . . . . . . . 51

3.3.2. Ejemplo: Viga en voladizo con carga armónica seguidora . . . . . . . . . . 54

4. Análisis determinista de la pérdida de pala $\quad 57$

4.1. Introducción . . . . . . . . . . . . . . . . . . . . . 58

4.2. Pérdida de pala del turbohélice . . . . . . . . . . . . . . . . . . 59

4.3. Descripción del modelo . . . . . . . . . . . . . . . . . . . . . . . . 61

4.4. Modelo FEM . . . . . . . . . . . . . . . . . . . . . . . 62

4.4.1. Cargas . . . . . . . . . . . . . . . . . . . 64

4.4.2. Unión bancada-ala . . . . . . . . . . . . . . . . . . 65

4.4.3. Unión motor-bancada . . . . . . . . . . . . . . . . . . . 65

4.4.4. Consideraciones de los análisis explícitos . . . . . . . . . . . . . . 68

4.4.5. Casos de carga analizados . . . . . . . . . . . . . . . . 71

4.4.6. Resultados de los casos de carga . . . . . . . . . . . . . . . 77

$\begin{array}{ll}\text { 5. Análisis aleatorio de la pérdida de pala } & 91\end{array}$

5.1. Introducción . . . . . . . . . . . . . . . . . . . . . . . 91

5.1.1. Ejemplo: Problema con variables aleatorias . . . . . . . . . . . . . . 92

5.2. Descripción del modelo . . . . . . . . . . . . . . . . . . . . . . 95

5.2.1. Reducción del modelo . . . . . . . . . . . . . . . . . . . . 95

5.2.2. La bancada . . . . . . . . . . . . . . . . . . . . 98

5.2.3. Consideraciones del análisis FEM; método implícito y explícito; paso de tiempo . . . . . . . . . . . . . . . . . . . . . 99

5.2.4. Cargas . . . . . . . . . . . . . . . . . . 103

5.3. Casos de carga analizados . . . . . . . . . . . . . . . . . . . . 103

5.3.1. Técnica del método de Monte Carlo (MCT) . . . . . . . . . . . . . . 103

5.3.2. Configuración de los parámetros de entrada . . . . . . . . . . . . . . . 104

5.4. Resultados . . . . . . . . . . . . . . . . . . . . . . . 108

5.4.1. Resultados preliminares de variaciones individuales con el modelo sólo motor 108

5.4.2. Resultados de variaciones individuales de los parámetros de entrada . . . 112

5.4.3. Interacción de todos los parámetros . . . . . . . . . . . . . . . . . . . 121

5.4.4. Resultados de la unión bancada-ala . . . . . . . . . . . . . . . . . . . . 129

5.4.5. Resultados de la secuencia de fallo . . . . . . . . . . . . . . . . . . 130 
6. Conclusiones y consideraciones 133

6.1. Conclusiones sobre el fenómeno de la pérdida de pala . . . . . . . . . . . . . . 133

6.1.1. Conclusiones del análisis con variables deterministas . . . . . . . . . . . . 134

6.1.2. Consideraciones acerca de los resultados deterministas . . . . . . . . . 135

6.1.3. Conclusiones del análisis con variables aleatorias . . . . . . . . . . . . 137

6.1.4. Consideraciones acerca de los resultados aleatorios . . . . . . . . . . . . . 140

6.2. Conclusiones generales . . . . . . . . . . . . . . . . . . . . . . 142

6.3. Consideraciones generales . . . . . . . . . . . . . . . . . . . . . 143

6.4. Otras consideraciones. Propuestas de futuros trabajos . . . . . . . . . . . . 143

$\begin{array}{ll}\text { Referencias } & 145\end{array}$

A. Contribuciones académicas $\quad 155$

A.1. Publicaciones propias de la tesis . . . . . . . . . . . . . . . 155

A.2. Publicaciones relacionadas con la tesis . . . . . . . . . . . . . 155

$\begin{array}{ll}\text { B. Medios y programas de software } & 157\end{array}$

B.1. Medios para la realización de la tesis . . . . . . . . . . . . . . 157

B.2. Flujo de integración en el programa Matlab . . . . . . . . . . . . . . . . 157

B.2.1. Datos y parámetros iniciales o constantes . . . . . . . . . . . . 157

B.2.2. Bucle principal . . . . . . . . . . . . . . . . . . 158

B.2.3. Datos de salida . . . . . . . . . . . . . . . . . . . . 159 


\section{ÍNDICE DE FIGURAS}

1.1. Clasificación de los problemas dinámicos . . . . . . . . . . . . . . . . . 3

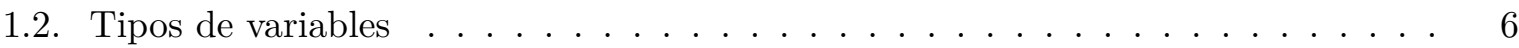

2.1. Problema de 2 grados de libertad . . . . . . . . . . . . . . . . . . . . . . 29

2.2. Tiempo de cálculo vs. incremento de tiempo en cada paso . . . . . . . . . . . 30

2.3. Resultados del nodo 1 (arriba) y nodo 2 (abajo) del método explícito . . . . . . . 31

2.4. Resultados del nodo 1 (arriba) y nodo 2 (abajo) del método implícito . . . . . . . 31

2.5. Desplazamientos en función de la frecuencia . . . . . . . . . . . . . . . 32

2.6. Desplazamientos en función del tiempo para el modo $1 \ldots \ldots$. . . . . . . . 33

2.7. Desplazamientos en función del tiempo para el modo 2 . . . . . . . . . . . . . . 33

3.1. Choque entre masas unidas por muelles . . . . . . . . . . . . . . . . 39

3.2. Posición en función del tiempo para $\Delta t 10^{-5}, 10^{-6}$ y $10^{-7} \mathrm{~s} \ldots \ldots \ldots$. . . . 40

3.3. Modelo de elementos finitos . . . . . . . . . . . . . . . . . . . . 42

3.4. Comparación entre el modelo FEM y los ensayos . . . . . . . . . . . . . . 42

3.5. La deflexión en función del tiempo para diferentes paneles y casos de carga . . . 43

3.6. Columna, impactador y base. Contacto impactador-columna y columna-base . . 47

3.7. Impacto a 50Km/h. Detalles de la colisión en base y columna . . . . . . . . . . . 49

3.8. Impacto a $140 \mathrm{Km} / \mathrm{h}$. Detalles de la colisión en base y columna . . . . . . . . . . 49

3.9. Energías de la columna e impactador . . . . . . . . . . . . . . . . 50

3.10. Fuerza del impacto . . . . . . . . . . . . . . . . . . . 50

3.11. Fuerzas aerodinámicas sobre las palas y simplificación del motor y sus apoyos . . 53

3.12. Viga en voladizo con carga armónica seguidora . . . . . . . . . . . . . . 54

3.13. Elementos de la viga y criterio de signos . . . . . . . . . . . . . 55

3.14. Desplazamiento nodos en función del tiempo . . . . . . . . . . . . . . 56

4.1. Fuerzas aerodinámicas sobre las palas de la hélice . . . . . . . . . . . . . . 60

4.2. Vibraciones en la aeronave (izquierda) y detalle del sistema de anclaje del motor

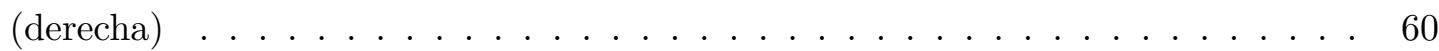


4.3. Sistema de anclaje del motor (EMS). Apoyos traseros o rear mounts"(izquierda) y superiores delanteros o "top mount"(abajo derecha) y laterales delanteros o "side mount" (arriba derecha) . . . . . . . . . . . . . . . . . 62

4.4. Propiedades elastoméricas . . . . . . . . . . . . . . . . . 63

4.5. Propiedades elásticas de los distintos materiales del modelo . . . . . . . . . . . 63

4.6. Modelo FEM y condiciones de contorno (unión bancada-ala puntos 1-4) . . . . . 64

4.7. Modelo de material perfectamente plástico . . . . . . . . . . . . . . 65

4.8. Evolución del modelo elastomérico y diagrama de muelles . . . . . . . . . . . . 66

4.9. Área de fallo . . . . . . . . . . . . . . . . . . . 68

4.10. Energías del modelo . . . . . . . . . . . . . . . . . . 71

4.11. Captura de la evolución habiendo excitado un modo propio . . . . . . . . . . . 71

4.12. Influencia de los distintos ángulos de la pala de la hélice perdida en las fuerzas . 73

4.13. Ejemplo de la estabilización antes de aplicar la carga . . . . . . . . . . . . . 74

4.14. Variación de la frecuencia rpm en las fuerzas en función del tiempo . . . . . . . . 75

4.15. Influencia de la forma y duración de la introducción de la carga en las fuerzas . . 75

4.16. Secuencia de fallos de los apoyos para un caso de carga (1 para el primer fallo,..., 6 para el último fallo) . . . . . . . . . . . . . . . . . . . 77

4.17. Fuerzas en las condiciones de contorno . . . . . . . . . . . . . 78

4.18. Elementos FEM (arriba) y elementos plastificados de un caso de carga (abajo) . 78

4.19. Tiempo de la simulación en los distintos casos de carga . . . . . . . . . . . . . . 79

4.20. Elementos plastificados en los distintos casos de carga . . . . . . . . . . . . 82

4.21. Ángulo y secuencia (rojo $1^{\circ}$, verde $2^{\circ}$, gris $3^{\circ}$, amarillo $4^{\circ}$, naranja $5^{\circ}$ y púrpura $6^{\circ}$ ) en los que se produce el fallo de los apoyos para cada caso de carga . . . . . . 83

4.22. Número de ciclos en los distintos casos de carga . . . . . . . . . . . . . . . . 85

4.23. Secuencia de fallo en los distintos casos de carga . . . . . . . . . . . . . 86

4.24. Energía del modelo en el momento del fallo de los distintos apoyos para todos los casos de carga . . . . . . . . . . . . . . . . . . . . . . 87

5.1. Correlaciones entre las variables $E, a d m_{2}, f_{1}$ y $f_{2} \ldots \ldots \ldots \ldots$

5.2. Histogramas y correlaciones entre variables de entrada y salida . . . . . . . . . 94

5.3. Esquema del modelo desde la hélice hasta el ala . . . . . . . . . . . . . . . . 96

5.4. Posiciones de los elastómeros en el modelo del capítulo 4 (final líneas rojas) y en este capítulo (flechas) . . . . . . . . . . . . . . . . . . . . 97

5.5. Posiciones de los apoyos en el modelo del capítulo (final líneas rojas) 4 y en este capítulo (flecha) . . . . . . . . . . . . . . . . . . . . 97

5.6. Rigidez (izquierda) y resistencia (derecha) de los apoyos . . . . . . . . . . . 98

5.7. Evoluciones de fuerzas X, Y y Z y los valores límites admisibles . . . . . . . . . . 99

5.8. Niveles de energía para el caso nominal . . . . . . . . . . . . . . . . . . . . . 101 
5.9. Energía total (izquierda) and detalle del error en la energía (derecha) para distintos esquemas y pasos de tiempo . . . . . . . . . . . . . . . . . . . 102

5.10. Resultados de la pérdida de pala para el modelo sólo motor (izquierda) e incluyendo la bancada (derecha) . . . . . . . . . . . . . . . . . . . 105

5.11. Resultados de tamaño de pala perdida y posición angular . . . . . . . . . . . 106

5.12. Resultados con variación de todos los parámetros de entrada . . . . . . . . . . 107

5.13. Matriz de correlación con varias variables de entrada y de salida de la simulación 110

5.14. Energía del modelo en el momento de la primera rotura vs. el ratio de pala no desprendida . . . . . . . . . . . . . . . . . . . 110

5.15. Distintas variaciones de parámetros vs. el ratio de pala no desprendida . . . . . . 111

5.16. Detalle de los resultados para variaciones individuales de los parámetros . . . . . 114

5.17. Ángulos probables de rotura para las variables SIZE, RPM y ALL en el modelo

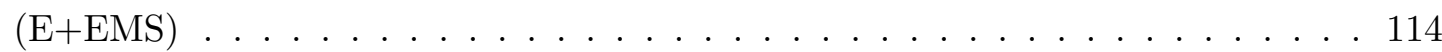

5.18. Variación de SIZE diferenciando con colores las distintas secuencias de fallo . . 115

5.19. Variación de RPM diferenciando con colores las distintas secuencias de fallo . . . 116

5.20. Variación de ANG diferenciando con colores las distintas secuencias de fallo . . . 117

5.21. Variación de STIFF diferenciando con colores las distintas secuencias de fallo . . 118

5.22. Variación de STR diferenciando con colores las distintas secuencias de fallo . . 119

5.23. Variación de DAMP diferenciando con colores las distintas secuencias de fallo . . 120

5.24. Resultados de FFE vs. SIZE variando sólo SIZE o SIZE más otro parámetro . . . 121

5.25. Detalle de filtrado de los resultados por SIZE (línea roja) o DAMP (línea naranja) 122

5.26. Variación de SIZE\&RPM diferenciando con colores las distintas secuencias de fallo123

5.27. Variación de SIZE\&ANG diferenciando con colores las distintas secuencias de fallo 124

5.28. Variación de SIZE\&STIFF diferenciando con colores las distintas secuencias de fallo . . . . . . . . . . . . . . . . . . . . . . . 125

5.29. Variación de SIZE\&STR diferenciando con colores las distintas secuencias de fallo 126

5.30. Variación de SIZE\&DAMP diferenciando con colores las distintas secuencias de fallo . . . . . . . . . . . . . . . . . . . . . . 127

5.31. Variación de TODO diferenciando con colores las distintas secuencias de fallo . . 128

5.32. Fuerzas máximas en el ala y los límites admisibles . . . . . . . . . . . . . . 130

6.1. Secuencias de la simulación . . . . . . . . . . . . . . . . . . . 136

6.2. Tiempo entre la primera y la última rotura vs. sólo la variación del ratio de pala perdida (izquierda) y con todas las variaciones de entrada (derecha) . . . . . . 138

6.3. Detalle de los resultados con variación de todos los parámetros de entrada . . . . 139

6.4. Resultados variando un parámetro de entrada en función de uno de salida identificando con colores las secuencias de fallo . . . . . . . . . . . . . . . . . . . . 141

6.5. Secuencias de fallos (colores) en función de la variación de dos parámetro de entrada141 
B.1. Flujo seguido por el programa . . . . . . . . . . . . . . . . . . 158 


\section{ÍNDICE DE TABLAS}

3.1. Propiedades de los materiales en mástil y base . . . . . . . . . . . . . . . 46

3.2. Los resultados más relevantes de la simulación . . . . . . . . . . . . . . . . . . . 48

4.1. Ciclos, tiempo analizado y paso de tiempo de mínimo en los casos de carga . . . 80

4.2. Intervalos de tiempos de fallos de los apoyos en los casos de carga . . . . . . . . . 81

4.3. Número de veces que rompe cada apoyo en un orden especificado . . . . . . . . . 82

4.4. Energía $[\mathrm{J}]$ del modelo cuando rompe el apoyo para todos los casos de carga . . . 84

5.1. Comparación de los modos propios entre modelos . . . . . . . . . . . . . 95

5.2. Esquemas de iteración . . . . . . . . . . . . . . . . . . . . . 100

5.3. Propiedades de las variables de entrada . . . . . . . . . . . . . . . . . . 104

5.4. Propiedades de los parámetros de salida . . . . . . . . . . . . . . . . 109

5.5. Probabilidad de fallo en los apoyos para cada grupo de casos de carga . . . . . . 130

5.6. Porcentaje de simulaciones que se han producido en una determinada secuencia de fallo por cada grupo de casos de carga . . . . . . . . . . . . . . . 131 


\section{INTRODUCCIÓN}

Índice

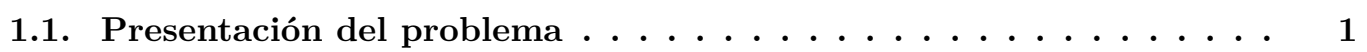

1.2. Ámbito científico de las herramientas ............. 4

1.2.1. Herramientas para el análisis de vibraciones . . . . . . . . . . . . . 7

1.2.1.1. Método de elementos finitos . . . . . . . . . . 7

1.2.2. Métodos de análisis paramétricos . . . . . . . . . . . . . . . 8

1.3. Estado de la técnica . . . . . . . . . . . . . . . . 11

1.4. Objetivos y alcance de la tesis . . . . . . . . . . . . 14

1.5. Descripción de la tesis $\ldots \ldots \ldots \ldots \ldots \ldots \ldots$

\subsection{Presentación del problema}

Un fallo estructural ocurre cuando la carga aplicada es superior a la capacidad resistente, bien de un componente, bien de la propia estructura. Estos problemas pueden derivar en un fallo catastrófico en el que se produce el fallo total del sistema y su recuperación es imposible. A lo largo de la historia han ocurrido gran variedad de estos tipos de fallo, el colapso del puente Tay Rail en 1879 cuyo problema fue debido a su diseño defectuoso como se indica en Eyre y Spottiswoode (1880), la rotura de la presa South Fork en 1889 por los mismos motivos, ver Unrau (1979), la caída del puente de Tacoma en 1940 por el desconocimiento del tipo de cargas a las que iba a estar sometido, ver Billah y Scanlan (1991), los desastres de los Comets en 1954 por la concentración de la carga, ver Withey (1997), los problemas en los lanzadores Shuttle,... Esto hace que se realicen multitud de análisis y ensayos antes de que una estructura se considere terminada. Durante un proyecto de avión, por ejemplo, existe una campaña de ensayos; primero a nivel de material si éste no está caracterizado al nivel necesario, a nivel probeta donde se analizan y ensayan las configuraciones que van a ser utilizadas, a nivel subcomponente donde se analizan y ensaya a rotura para ver su capacidad estructural, y a nivel de componente o estructura completa en donde se ensayan distintos casos de los requisitos de la estructura. Esta es una misión, por ejemplo, del departamento de materiales y estructuras del INTA que con más de 50 años de experiencia ofrece instalaciones para el análisis y realización de ensayos 
estáticos, dinámicos y de fatiga de estructuras completas, fabricadas con materiales compuestos y/o metálicos.

Por todos los sucesos anteriormente mencionados, aun realizando multitud de análisis y ensayos, son necesarios unos factores de seguridad que nos salvaguarden de las fuentes de incertidumbres que afectan a los problemas ingenieriles. Estas incertidumbres pueden ser de muy distintos tipos y pueden afectar a parámetros tan importantes como las propiedades del material, a las cargas de la estructura, a la propia modelización del problema, a las incertidumbres en las estadísticas, a las decisiones, a los errores humanos y a casi cualquier fenómeno, como se pone de manifiesto en Maymon (1993). La fiabilidad de las estructuras permite algunas de estas incertidumbres, si éstas están cuantificadas y señaladas explícitamente en los análisis. Debido a estas incertidumbres en los análisis se mide la seguridad estructural, la probabilidad de fallo. Las incertidumbres en las características estructurales y en las cargas se cuantifican utilizando teorías matemáticas de probabilidad, variables aleatorias, procesos aleatorios y estadísticos. Uno de los grandes objetivos perseguidos es conocer la relación entre la probabilidad de fallo estructural y la incertidumbre de esos parámetros. Los métodos de análisis ayudan a elegir la configuración adecuada y los factores de seguridad que se deben utilizar en un proyecto para un nivel de seguridad y para un coste determinado. Estos análisis son aún más importantes en estructuras atípicas o novedosas donde no se pueden aplicar los estándares de diseño o en las que se corren grandes riesgos como centrales nucleares, presas, estructuras bajo cargas sísmicas, ... Asimismo estos métodos son fundamentales en el estudio de cargas y fallos estructurales complejos en el ámbito aeroespacial, como el espectro de cargas en un tren de aterrizaje, la inestabilidad debida a la pérdida de pala de una hélice o, en el ámbito automovilístico, el choque de un coche.

Se han realizado estudios en este campo utilizando los métodos de probabilidad y estadística en los factores de seguridad. En este punto cabe destacar el trabajo de Rackwitz (2000) en donde se presenta una revisión de la fiabilidad de los métodos estructurales. En lo que respecta a los análisis, en el siglo pasado se fue progresivamente mejorando la teoría de los fenómenos aleatorios y se implementaron nuevos métodos de caracterización: análisis de Fourier, funciones de autocorrelación y funciones de densidades espectrales. Aparecieron métodos espectrales que se aplicaron a las ecuaciones del movimiento de sistemas complejos. A finales de los 50 surgió la necesidad de una teoría coherente acerca de las vibraciones aleatorias. Cabe destacar el primer libro de Crandall y Mark (1963) acerca de esta teoría. En los años 60 la teoría se extendió a gran variedad de sistemas dinámicos. Es importante señalar el desarrollo de la trasformada de Fourier discreta de Cooley y Tukey (1965) que permitió implementar la formulación en los ordenadores. También los artículos de Ibrahim y Manohar (1999) que dan una visión global de los problemas estructurales dinámicos con incertidumbre en los parámetros. En la figura 1.1 se muestra una clasificación de los problemas dinámicos según el tipo de análisis que se pueden aplicar.

Como se ha comentado, la respuesta de las estructuras se empieza a relacionar con el concepto de fiabilidad y con su cálculo. En Mahadevan y Chamis (1993) se aplica a un sistema de 


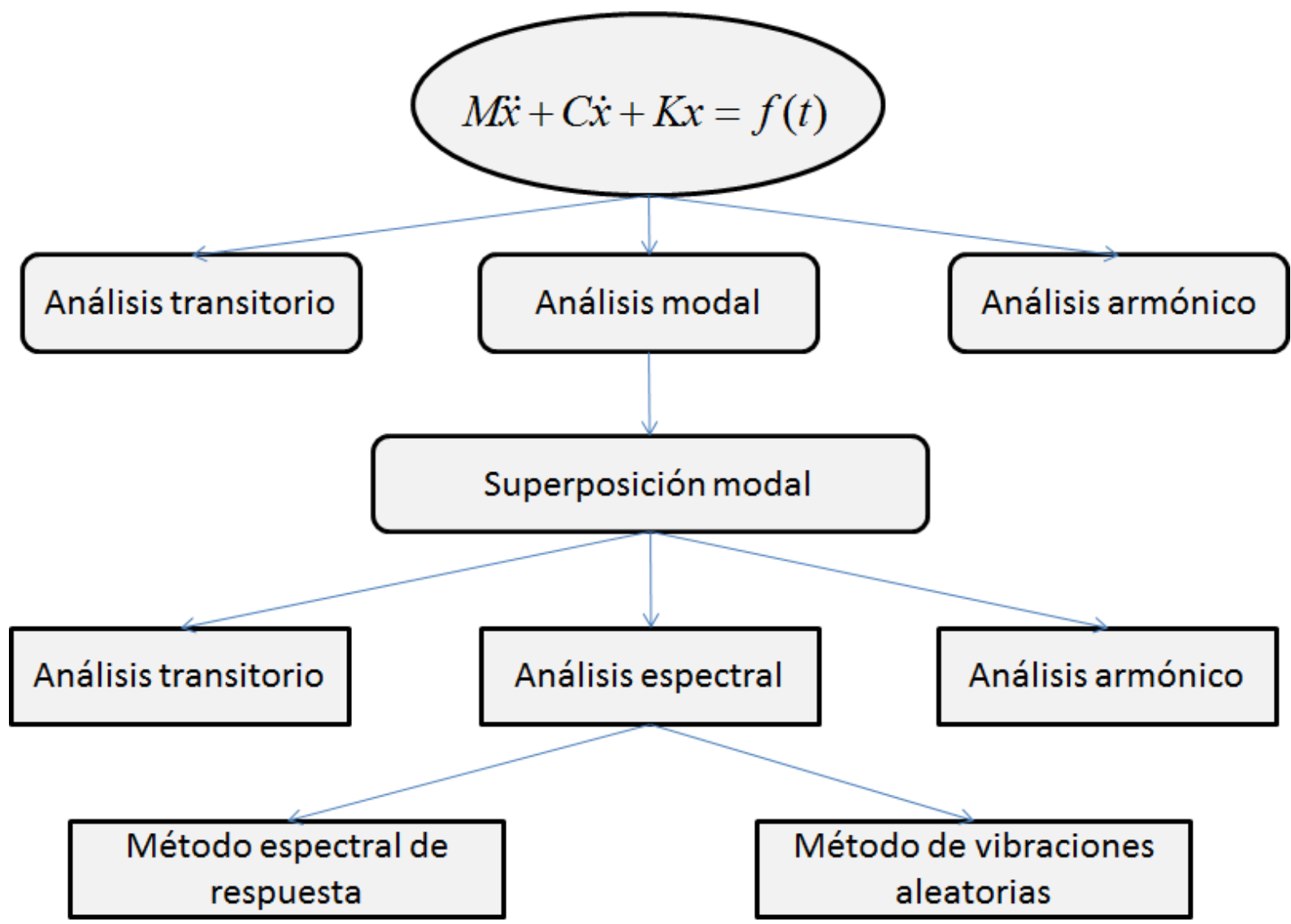

Figura 1.1: Clasificación de los problemas dinámicos

dos rotores, este sería un ejemplo de un sistema complejo en el que se quiere calcular la fiabilidad del sistema ante un fenómeno. Se empiezan a estudiar distintos métodos para el cálculo de la fiabilidad bajo distintas condiciones: para variables correladas como en la referencia Nikolaidis et al. (1995) donde se aplican distintos algoritmos (el de Rackwitz-Fiessler y el de KiureghianLieu's) que se pueden aplicar cuando la información existente se limita a la distribución de probabilidad marginal, en determinadas estructuras como es el caso de Maymon (1999) en donde se estudia la probabilidad de fallo de un motor de lanzamiento de satélites con determinadas imperfecciones geométricas, y analizando los riesgos como en Sørensen (2004) donde se realiza una revisión de los distintos métodos aplicándose tanto a sistemas en serie como en paralelo. Otras revisiones cronológicamente ordenadas del análisis de la fiabilidad se realizan en Rackwitz (2000), en Webster y Bannister (2001), Manohar y Gupta (2002) y Ditlevsen y Madsen (2007).

Los métodos evolucionan y no sólo se considera un único tipo de fallo sino que se empiezan a considerar múltiples como en la tesis de Adduri (2006) en donde se plantean numerosos ejemplos de aplicación. Merece la pena destacar aplicaciones enfocadas al estudio de la propagación de grietas incluyendo la estimación del fallo como en Maymon (2003). Este mismo autor llega a incluir también incertidumbres en el proceso, Maymon (2005), tanto en la estimación de 
propagación de grietas como en el cálculo de autovectores; también cabe destacar el estudio probabilístico de la fiabilidad de una estructura tolerante al daño desarrollado en Wu y Shin (2005). Para terminar en Lee y Hwang (2008) se revisan las técnicas de estimación de la probabilidad de fallo señalando los avances y retrocesos de las mismas.

Es importante señalar que las autoridades también investigan y publican estudios acerca de los fenómenos que aquí se tratan como, por ejemplo, sobre la contención de los álabes de turbina, Administration (1990) o la fatiga que se produce en las palas, Administration (2001). También se estudian los fenómenos desde el punto de vista de su diseño como las cargas aerodinámicas de una turbina (carga aleatoria) en Fitzwater y Winterstein (2001); Veers y Butterfield (2001); Fitzwater y Cornell (2003). En la tesis Parthasarathy (2003) se estudia el fenómeno de la pérdida de pala para la dinámica del rotor.

Para terminar, comentar que toda esta mejora en los métodos de análisis de estructuras está permitiendo analizar sistemas que hace unos años eran impensables. Todo el desarrollo y cálculo de los aviones se basaba en análisis estructurales sencillos, en la experiencia en aviones similares y en baterías complejas de ensayos. Un ejemplo de actualidad es el Airbus A400M, avión militar de transporte, y más en particular los análisis y ensayos de su motor Europrop TP400. Las metodologías anteriormente mencionadas están permitiendo abordar el estudio de fenómenos que pueden llevar al fallo catastrófico de la estructura como pueden ser el impacto de pájaro o la pérdida de pala de la hélice que se estudia en detalle más adelante. Este tipo de análisis se debe abordar mediante un método de vibraciones aleatorias que permite implementar el tipo de cargas y las incertidumbres que se presentan en estos problemas para poder así atacar todas las configuraciones estructurales. Actualmente distintos centros de investigación están estudiando y tratando de adquirir conocimiento en este tipo de fenómenos y en la forma de analizarlos, en este caso el INTA y la UPM han colaborado con EADS-Casa para el estudio de estos posibles fallos estructurales como se puede ver en las referencias de A.1.

A continuación se realiza un acercamiento más profundo del ámbito científico de la tesis propuesta. Se plantean las formulaciones para los análisis, las herramientas para la realización de los mismos, los métodos de análisis paramétricos y se introducen fenómenos en los que más adelante se profundizará. En general se repasa el estado del arte y la situación actual y una vez planteado el escenario se marcan unos objetivos.

\section{2. Ámbito científico de las herramientas}

Una vez introducidos los tipos de análisis para abordar los problemas dinámicos nos centramos en las formulaciones existentes cuando se estudian diferentes parámetros en problemas estructurales con cargas y/o propiedades, tanto deterministas como aleatorias. Conocer con el mayor detalle posible la respuesta de los parámetros de estudio capacita para aproximarse a problemas estructurales difícilmente abordables en la actualidad aplicando las teorías existen- 
tes, las dificultades pueden venir tanto de las cargas como de las características de la estructura a estudiar. Los distintos tipos de cargas no son de igual importancia y dependen del tipo de estructura mecánica a la que estén sometida, no es lo mismo una estructura primaria que no puede permitir de ninguna manera el fallo que secundaria que sólo debe cumplir que en caso de fallo, el fallo no debe tener consecuencias catastróficas para la estructura. Los requisitos están especificados para cubrir las cargas a las que va a estar sometida la estructura durante su vida útil:

- Frecuencias naturales. Requisito imprescindible del diseño de las estructuras primarias.

- Aceleraciones. Se analiza según sean los requisitos de la estructura.

- Cargas semi estáticas y excitaciones sinusoidales de baja frecuencia. Requisito imprescindible del diseño de las estructuras primarias.

- Excitaciones sinusoidales y aleatorias. Suele ser requisito de ciertas estructuras secundarias sobre todo en el campo espacial.

- Ruido. Requisito estructural para estructuras ligeras de gran superficie del campo espacial.

- Cargas transitorias. Se analiza según sean los requisitos de la estructura.

- Choques. Se analiza según sean los requisitos de la estructura.

- Temperaturas. Se analiza según sean los requisitos de la estructura.

Por lo tanto existen muchos tipos de posibles análisis a realizar en las estructuras:

- Vibraciones de sistemas dinámicos mecánicos lineales deterministas bajo cargas mecánicas aleatorias y desplazamientos forzados (aceleraciones). Se suelen analizar mediante métodos de desplazamientos modales, muy utilizados en el método de elementos finitos (FEM). Suelen ser vibraciones de baja frecuencia y la teoría utilizada la de un sistema de un grado de libertad.

- Vibraciones de sistemas dinámicos mecánicos lineales deterministas bajo cargas acústicas aleatorias. Se suelen utilizar métodos análogos a los anteriores, sin embargo, la carga suele estar aplicada en toda la superficie de la estructura mecánica.

- Vibraciones aleatorias de sistemas mecánicos lineales. Se suelen realizar métodos de análisis estadísticos de energía (SEA), que es un método estadístico para determinar el intercambio energético entre diversos sistemas, en donde las cargas son de mayor frecuencia y se suelen tratar de una manera estadística, dependiendo del problema el método de elementos finitos es otra vía de modelización posible. 
- Estructuras no-lineales bajo cargas mecánicas aleatorias y analizadas mediante ecuaciones diferenciales estocásticas. Se basa en métodos gaussianos, ruidos blancos. Medias, funciones de correlación, métodos estadísticos son utilizados en estas teorías. Se necesita mucho tiempo de cálculo para resolver las ecuaciones. Se suele restringir a sistemas dinámicos no-lineales de pocos grados de libertad.

Sin embargo todos estos análisis estructurales tienen una serie de parámetros fundamentales en el problema que condicionan la respuesta. Las incertidumbres de estos parámetros se pueden cuantificar utilizando modelos matemáticos y teorías de probabilidad tanto para procesos deterministas como aleatorios. El objetivo de estos análisis es conocer la relación entre la probabilidad de que uno de estos parámetros llegue a un cierto umbral y las características estructurales y de cargas. En la figura 1.2 se muestra una clasificación por tipo de variable. En el caso de conocer estas relaciones se podría diseñar la configuración estructural óptima para conseguir el comportamiento deseado en el parámetro estudiado.

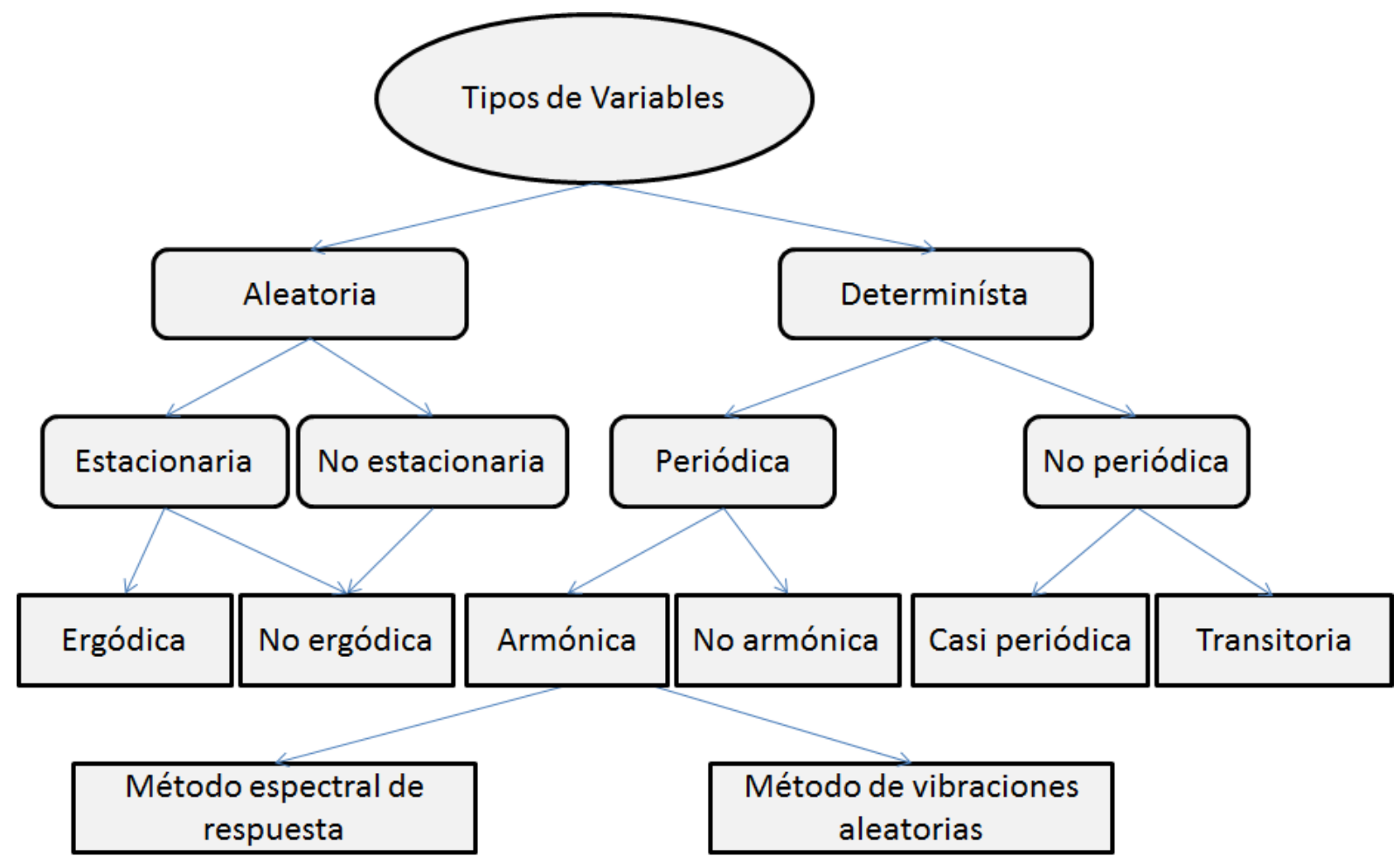

Figura 1.2: Tipos de variables

En los casos siguientes interesa, ya que condicionaran la repuesta del sistema, el comportamiento de los siguientes parámetros:

- Choque de masas. Relación entre la fuerza que se produce en el choque con la velocidad de las masas.

- Impacto. Relación entre la energía del impacto y la resistencia residual de la estructura. 
- Estructura frangible. Relación entre la velocidad a la que se produce la colisión y la energía que se pierde en la misma.

- Viga con carga armónica. Relación entre los parámetros de la carga y los desplazamientos y esfuerzos de salida.

- Pérdida de pala. Relación entre los parámetros de la carga y otras variables del vuelo en el momento del incidente y los desplazamientos, fuerzas y esfuerzos de salida en diferentes partes de la estructura.

\subsubsection{Herramientas para el análisis de vibraciones}

Una vez presentados los tipos de problemas dinámicos hay que estudiar los distintos métodos de análisis para el estudio de su respuesta. Las frecuencias de interés del análisis van a ser fundamentales para la elección del método. Cada método tiene un determinado rango de frecuencias donde las hipótesis de la correspondiente formulación se verifican en todo momento. En general, cuanto menor es la frecuencia menor es la densidad modal. Por ese motivo cuando la densidad modal es baja el uso del método de los elementos finitos (FEM) combinado con el de los elementos de contorno (BEM) es muy adecuado. Sin embargo a medida que la densidad modal se incrementa estos métodos necesitan un mayor número de elementos para conseguir la misma precisión, por lo que el esfuerzo computacional aumenta. Asimismo, la formulación deja de ser adecuada y pequeños detalles influyen en la respuesta del sistema. En cambio, cuando la densidad modal es alta la formulación de los métodos energéticos (SEA) se muestra más apropiada. La eficiencia de esta formulación baja cuando existe acoplamiento entre los subsistemas. El esfuerzo de cálculo de estos elementos para frecuencias altas es mucho menor que en los demás métodos. En la actualidad se están realizando muchos estudios sobre métodos híbridos en donde existen elementos modelizados por el método de elementos finitos y elementos modelizados por métodos estadísticos de energía, cada uno en la formulación más adecuada a sus características. Un ejemplo se puede ver en el artículo de Troclet et al. (2009). El objetivo común de todos estos métodos radica en tratar de obtener el mismo comportamiento que en la realidad de la magnitud física de interés.

\subsubsection{Método de elementos finitos}

Turner et al. (1956) trabajando en Boeing desarrolló la formulación precursora FEM para problemas lineales, el método avanzó en las universidades para resolver problemas estáticos no lineales con pequeños desplazamientos. Los primeros códigos que se desarrollan son implícitos que resuelven las ecuaciones en el propio y en el siguiente paso de integración. En la década de los 60 se introduce el aspecto no lineal en los materiales y a finales se avanza en la complejidad de los algoritmos para simular los comportamientos no lineales. En ese tiempo nace el código 
que más adelante se utilizará en algún ejemplo, MARC. En 1972 se funda otro de los códigos más conocidos ABAQUS y ese mismo año MSC comercializa el NAsaSTRucturesANalysis, NASTRAN. En lo que respecta a los códigos explícitos, que resuelven las ecuaciones para el siguiente paso de integración, nacen un poco después pero también en la década de los 60 , por ejemplo Constantino en el IIT Research Institute. Belytschko plantea su teoría del elemento-libre por el método de Galerkin a finales de la década y principios de la siguiente, en ese tiempo sólo se utiliza para aplicaciones nucleares, más adelante formularía el método de elementos finitos extendido. En los años 70 nace DYNA que incluye contactos, en 1980 PAMCRASH y es en 1990 cuando MSC comercializa DYTRAN inicialmente orientado a resolver problemas de interacción fluido estructura. Los métodos de elementos finitos evoluciona de maneras distintas, una de ellas es la de dejar de considerar el cálculo determinista e introducir el probabilístico como en Cesare y Sues (1999), o estocástico, Ghanem y Sarkar (2001). Otra aplicación típica de cálculo de la fiabilidad de una estructura con métodos de elementos finitos deterministas se encuentra en Beck y da Rosa (2006) donde se utiliza en ejemplos numéricos de esfuerzos en estructuras, en particular puentes. En este caso se ha incorporado los algoritmos de fiabilidad estructural en un código de software determinista de elementos finitos. Otro método que se debe mencionar es el método estadístico de energía cuya aplicación es adecuada para grandes frecuencias como ya se ha mencionado, véase un ejemplo en Troclet et al. (2009).

En este punto debemos mencionar el trabajo realizado por Craig y Bampton (1968) que permite simplificar y reducir estructuras grandes en subestructuras utilizando las matrices de masa y rigidez. Este trabajo permitió abordar problemas antes imposibles, así como trabajar en paralelo en diferentes subestructuras. Esta técnica siguió evolucionando, un ejemplo se puede ver en Rixen (2004) donde extiende la técnica mediante nuevas fuerzas en las interfaces que conlleva matrices reducidas más sencillas, y optimizándose llegando a incluirse en los softwares comerciales, ver MSC (2005).

Finalmente y enlazando con los métodos de análisis paramétricos y de cálculos de fallo se puede señalar la referencia Sudret (2002) donde se comparan distintos métodos de elementos finitos para estos cálculos de fiabilidad, o la referencia Sarkar y Ghanem (2001) que utiliza un modelo de elementos finitos estocástico para poder incluir las incertidumbres en los parámetros que en este caso se han considerado gaussianas, término que se explica más adelante. También se realiza este tipo de análisis estructurales probabilísticos en la referencia Huh y Haldar (2011). Por lo tanto, el método de elementos finitos se va a utilizar como una herramienta más a la que se van a aplicar metodologías adicionales.

\subsubsection{Métodos de análisis paramétricos}

Una forma de realizar los análisis paramétricos es de manera analítica resolviendo directamente las ecuaciones paramétricas. En lo que respecta a la bibliografía de este tema un libro 
muy interesante y con numerosas aplicaciones de vibraciones aleatorias es el Maymon (1998) y una revisión de las aplicaciones de las vibraciones aleatorias se lleva a cabo en Paez (1999). Otros libros recomendables acerca de las vibraciones aleatorias donde se pueden ver tanto desarrollos teóricos como aplicaciones prácticas son los Roberts y Spanos (1999); Wijker (2004); Wirsching et al. (2006); Wijker (2009), la colección de tomos Lalanne (2009) o el práctico Harris y Piersol (2002). Un libro que muestra las propiedades de las señales es el Gray (2010). El desarrollo teórico va evolucionando y se empiezan a considerar en el análisis los procesos gaussianos, que son los que cumplen con el teorema central del límite. Este teorema dice que si $S_{n}$ es la suma de $\mathrm{n}$ variables aleatorias independientes y de varianza no nula pero finita, entonces la función de distribución de $S_{n}$ 'se aproxima bien' a una distribución normal (también llamada distribución gaussiana, curva de Gauss o campana de Gauss). Éste es el caso de Sondhi (1983) o Man. También se desarrollan métodos para incorporar los fenómenos con comportamientos no gaussianos como en Ghanem et al. (2007) donde se trata de introducir la formulación necesaria para completar los análisis deterministas en los que no se conoce toda la información de algún parámetro. El conocer ya las herramientas que se han mencionado conllevó a que se introdujeran conceptos nuevos, aparecen métodos para determinar el primer paso por un valor determinado denominado umbral como en Zibdeh y Heller (1986) donde se utiliza en motores cohete con combustible sólido. Este concepto resulta muy útil para el análisis estructural ya que si ese valor resulta ser el admisible se convierte en el propio cálculo estructural en donde se determina si las cargas producen valores mayores a los admisibles.

Otro concepto interesante es el valor o valores máximos que alcanza un función, si esto se aplica al cálculo estructural tanto las cargas como los valores extremos se tratan como funciones, como se realiza en Laino y Pierce (2000), o en referencias más recientes que incluso clasifican los tipos de valores extremos, Fraga y Neves (2010), o la referencia Nadarajah (2003) donde se identifican el tipo de valores extremos de más de 30 distribuciones. Con este concepto se podrían identificar los valores máximos de una magnitud de interés en función de las variables de entrada. Otra aplicación que conjuga tanto el concepto de valor umbral como el de valores extremos es la de Rootzén y Tajvidi (2006).

Se empiezan a simular también fenómenos no normales como en Gurley et al. (1996) donde se aplica a un sistema de redes neuronales, o en Trandafir y Demetriu (2004) en donde se aplica para la simulación de vientos, olas y terremotos. En este punto hay que señalar la revisión llevada a cabo en Ibrahim y Manohar (1999) acerca de la introducción de incertidumbres en los parámetros en la dinámica estructural que resulta de gran interés ya que son aplicaciones que se van a utilizar más adelante.

En un ámbito más propio del cálculo estructural, otra evolución de la técnica es estudiar la masa y la rigidez para que se obtengan modos propios de una manera aleatoria lo que implica tratar variables estructurales con estas técnicas, como en Manohar y lyengar (1993) que se aplica a elementos muy sencillos de una dimensión, o en Ishida (2001) que lo aplica a vigas de sección 
circular, en Adhikar y Friswell (2004) se aplica a sistemas masa-muelles de varios grados de libertad y se compara con la técnica de Monte Carlo que más adelante se detalla y se utiliza. Este mismo autor realiza la misma comparación para el caso de distribuciones más genéricas, Adhikar (2005).

Finalmente y para unir con las teorías más utilizadas de los elementos finitos en el caso de incertidumbres en el modelo matemático esto puede afectar tanto a los modelos de las leyes de material (linealidad, isotropía, homogeneidad), relación esfuerzo-deformación (lineal/no-lineal) como a las teorías tan utilizadas como la de Euler-Bernoulli o Timoshenko de vigas, KirchoffLove o Mindlin de placas. Otros ejemplos son los modelos de amortiguación (viscoso/estructural), interacción entre los modelos de elementos mixtos (FEM/BEM), elección de la malla en los modelos FEM o los errores numéricos. En la referencia Mazur-Sniady et al. (2005) se aplican incertidumbres en los parámetros tanto variables dinámicas como estructurales en vigas bajo carga estocástica. Más referencias se han mencionado en el apartado 1.2.1.1. Todas estas aplicaciones mostradas en las referencias permiten que hayamos podido introducir incertidumbres en nuestro modelo matemático en particular, tanto en variables como la rigidez o la resistencia (ambas relacionadas con las leyes de material y la relación esfuerzo-deformación), el amortiguamiento estructural como las propias cargas aplicadas.

Por lo tanto se han planteado muchos ejemplos en donde se realizan los análisis paramétricos de una manera analítica. Sin embargo en la práctica no resulta abordable debido a las no-linealidades del problema que pueden ser tanto estructurales, geométricas, de material, como de las cargas aplicadas. En este caso las simulaciones mediante la técnica de Monte Carlo puede ser una forma de realizar estos análisis. Estas simulaciones utilizan el fenómeno, que se utiliza continuamente en estadística, de que un parámetro puede expresarse como la media de un gran número de resultados aleatorios de ese mismo parámetro. La idea fundamental consiste en resolver una batería de problemas mediante procedimientos deterministas para conocer los valores que toma el parámetro.

Al contrario de los métodos analíticos que sólo se puede utilizar en fenómenos muy determinados, los métodos computacionales no tienen esas limitaciones y cualquier tipo de estructura, geometría, material, y carga aplicada puede aproximarse. En los últimos años se han estudiado en profundidad los problemas estructurales con características de masa, geometría y/o excitaciones con comportamiento aleatorio, que como se verá más adelante es considerar como variables aleatorias las variables de la ecuación 2.3. Las matrices estructurales han pasado a ser función de la discretización de estas variables aleatorias. A partir de aquí surgen distintas formas de tratar los autovalores de la matriz aleatoria, la inversa de la matriz aleatoria y la solución de la matriz aleatoria de las ecuaciones diferenciales, que son las que determinan los autovalores y autovectores de los modos propios que a su vez son críticos para conocer el comportamiento estructural del sistema. 
Para terminar, se destacan una serie de referencias donde se utiliza el método de Monte Carlo. Por ejemplo en el problema de la introducción de las cargas aleatorias que se plantea en Shinozuka y Yang (1971) donde se empieza a tratar la respuesta dinámica como señales aleatorias no estacionarias, finalmente este mismo autor utiliza la técnica de Monte Carlo para su resolución en Shinozuka y Wen (1972). El estado de esta técnica de Monte Carlo va evolucionando y aplicándose a distintos ámbitos como por ejemplo en la vibración de una placa flexible en Vaicaitis et al. (1972). Se empieza a utilizar para el cálculo de la respuesta de estructuras frágiles como en Mahadevan y Dey $(1996,1997)$ donde sirve para identificar el mecanismo de fallo más probable, o para el análisis de autovalores como en Ghosh et al. (2005) donde se compara la aproximación con esta técnica con otra basada en las series de Taylor. Más adelante este mismo autor en Ghosh y Ghanem (2005) utiliza nuevos algoritmos con esta técnica para el cálculo de autovalores. Aplicaciones más recientes de esta técnica se pueden ver en la sección 1.3. Todas estas aplicaciones permiten que hoy en día se utilice la técnica de Monte Carlo para casi cualquier ámbito con resultados muy precisos y prometedores que hacen que sea cuestión de tiempo que se extienda aun más, como sucede en esta tesis como se ve más adelante.

\subsection{Estado de la técnica}

Existe una gran variedad de métodos analíticos y de simulación para el análisis estructural. Aparte del reducido interés de los métodos analíticos para ciertas aplicaciones los métodos de simulación están realizando grandes avances. Los desarrollos de metodología gaussiana/nogaussiana, escalar/vector, variables aleatorias/campos aleatorios y la mejora en los ordenadores actuales lo está permitiendo. Una de las desventajas de estos métodos está relacionada con el error numérico cuando se simulan fenómenos complejos y extremadamente no-lineales. En el pasado reciente los esfuerzos se centraron en el estudio de funciones concretas asociadas a componentes estructurales individuales como se puede ver en 1.2.2. Los desarrollos más recientes muestran que el análisis de un fallo estructural requiere el estudio de varias funciones de estado simultáneamente, como se ve en Gupta (2004), teniendo en cuenta las peculiaridades del problema como nuevos caminos de carga y/o estructuras redundantes. Con lo mencionado en los apartados anteriores en la actualidad se pueden decir que se han realizado los siguientes avances importantes en el estudio paramétrico dentro del análisis estructural:

- Disponibilidad de herramientas contrastadas en la simulación de problemas estructurales.

- Generalización de procedimientos deterministas robustos como el método de elementos finitos para la simulación y estudio de parámetros con incertidumbres.

- Formulación del problema estructural con variables no-gaussianas. Desarrollo de los métodos, describiendo, simulando y discretizando el campo no-gaussiano aleatorio. 
Además se han identificado los siguientes temas como líneas de investigación:

- El uso de la teoría de valores umbral en el análisis estructural, casos en los que existan varias variables con valores que necesitan estar dentro de un rango. En el caso de análisis aleatorios con un acercamiento estadístico aparecen funciones de probabilidad y correlaciones debido a la interacción entre las variables. Este tipo de análisis no se ha estudiado en profundidad.

- Análisis estructurales con incertidumbres en distintas variables que añaden complejidad y que habitualmente se suelen despreciar. Los procedimientos deben incorporar incertidumbres en los parámetros para ver su influencia en la respuesta.

- La discretización de campos aleatorios no-gaussianos para el que se definiría un vector de variables aleatorias que tuviera las características y propiedades del campo no-gaussiano.

Recientemente para el abordaje del estudio de estas líneas de investigación surgen distintos técnicas, cuya mayoría se han introducido en el apartado 1.2.2, como es la teoría de los valores máximos en donde se determina la probabilidad de la distribución de alcance de valores máximos. Esta teoría nos permite el tratamiento del problema incluyendo la variable temporal cuando interesa que no se alcance un valor en un determinado tiempo. La teoría de los valores máximos se trata en referencias desde un punto vista matemático, como por ejemplo en Gupta y van Gelder P.H.A.J.M. (2007) donde se estudia los valores extremos en procesos gaussianos con no linealidades, o desde un punto de vista más práctico como en Lu (2008) donde se estudian vigas y placas no lineales con cargas aleatorias. De una manera similar se puede atacar el problema con el concepto de valor umbral y las veces en las que una función sobrepasa cierto umbral, en este caso se puede mencionar la referencia Song y Der Kiureghian (2006) que lo hace desde un punto de vista genérico y compara los resultados con los obtenidos con la técnica de Monte Carlo. En el problema de un parámetro continuo y estacionario este fenómeno se corresponde con el momento en que el parámetro cruza cierto valor umbral, y bajo ciertas condiciones se puede modelizar como un proceso de aproximación de Poisson, tanto para procesos aleatorios gaussianos como los no-gaussianos como se trata en Leira (2003). Una tesis interesante sobre el análisis de valores extremos es la de Nolde (2010) que resume y desarrolla convenientemente los conceptos aquí presentados. El problema de determinar la distribución de alcance de valores máximos puede complicarse si se tiene en cuenta una serie de variables aleatorias dependientes entre ellas. Esto es debido al hecho de que se debe calcular la distribución conjunta de la serie de variables aleatorias. Cabe destacar que contrariamente al caso de una única variable independiente en donde existe una única distribución en este problema las distribuciones son dependientes para la serie de las distintas variables aleatorias, se trata de variables con correlación. En algunos casos y dependiendo de la estructura se ha determinado que bajo ciertas condiciones las distribuciones son idénticas. Sin embargo ciertos estudios han señalado que una pequeña dependencia aparen- 
temente despreciable puede derivar en grandes diferencias. En referencias recientes Roscoe y Diermanse (2015) se realiza un estudio de la fiabilidad de sistemas con componentes correlados, también en la referencia Lin y Zong (2015) se estudian variables correladas en un análisis de un puente por el modelo de elementos finitos. Otro de los problemas importantes al que nos enfrentamos en este tipo de análisis es la incertidumbre en las variables. Estas incertidumbres pueden tener distintas fuentes; pueden ser incertidumbres inherentes o físicas, incertidumbres del modelo, en la estimación del error o incluso debidas a factores humanos. Y esto puede ocurrir tanto en propiedades estructurales como en las características de la carga. Estas incertidumbres pueden ser debidas al desconocimiento del comportamiento estructural o a las simplificaciones introducidas por los modelos. Por estos motivos es importante reducirlas lo máximo posible conociendo y estudiando el fenómeno físico del problema. En el caso de incertidumbres en la estimación de los errores se debe reducir mediante la correcta aplicación del modelo estadístico. Esto afecta a la fiabilidad de un sistema como se estudia en la referencia Der Kiureghian (2008) donde se realiza un análisis estructural más bien teórico en el que se calcula el índice de fallo de una estructura con incertidumbre en los parámetros. En este punto hay que destacar la tesis Gupta (2004) sobre esta temática que revisa la literatura, aplica el concepto de valores extremos en distintos ejemplos y realiza análisis de fiabilidad y fallo en distintas situaciones y teniendo en cuenta incertidumbre en los parámetros.

El estudio estocástico de parámetros se continúa estudiando en la literatura como en Lei y Qiu (2000) en el que se incluyen comportamientos no-lineales, y evoluciona llegando incluso a aplicaciones como el cálculo residual de estructuras mediante lógica difusa como en la tesis Faust (2003). En la actualidad se siguen publicando artículos de procesos estocásticos con resoluciones analíticas como en Adhikar (2007b) y Adhikar (2008), además este mismo autor tiene en cuenta en sus estudios incertidumbres en los parámetros en Adhikar (2007a).

En la literatura se pueden ver estudios para obtener los procesos aleatorios no-gaussianos gracias a filtros en el ruido blanco aplicando la teoría de Markov como en Sondhi (1983), distintas variables aleatorias aplicadas a modelos estructurales como en Sobczyk et al. (1996). Los modelos no-gaussianos se pueden complicar para varias variables como se puede ver en Ditlevsen y Madsen (2007), y se pueden aplicar distintos modelos a fenómenos no normales como pueden ser modelos aerodinámicos que incluyen la pérdida como se ha llevado a cabo en Bertagnolio et al. (2008), o en sistemas que sufren degradación como en Sobczyk (2006) que incluye una degradación de la rigidez por fatiga.

Para terminar se vuelve a enlazar con la técnica de Monte Carlo que se sigue extendiendo y se utiliza para el cálculo de la respuesta de un sistema con incertidumbres en los parámetros como en Masri et al. (2006), y también para la estimación de la integridad estructural como en Hoitsma et al. (2010). Se utiliza también en conjunción con el método de elementos finitos en aplicaciones sencillas de placas Dhainaut et al. (2007) o de vigas Praks y Brozovsky (2010). En Adhikar y S. (2010) se realiza varias investigaciones experimentales y se comparan con los 
resultados numéricos obtenidos de la técnica de Monte Carlo en vigas y placas para el cálculo de modos propios.

\subsection{Objetivos y alcance de la tesis}

El primer objetivo es analizar la validez de las formulaciones existentes cuando se estudian diferentes parámetros en problemas estructurales con cargas y/o propiedades tanto deterministas como aleatorias altamente dinámicas y no-lineales. Éste es el caso de los elementos estructurales típicos (vigas y placas) empleados en la modelización de las estructuras aeroespaciales con las propiedades y cargas a las que están sometidas. El objetivo fundamental es el estudio y aplicación a un sistema mediante la combinación de estos elementos, mejorando el conocimiento de la respuesta de los parámetros de interés, así como desarrollando modelos optimizados sin penalización en los mismos, como por ejemplo sería un tiempo requerido de cálculo excesivo o falta de convergencia o poca fiabilidad de la solución. La finalidad es la aproximación a problemas estructurales difícilmente abordables en la actualidad aplicando las teorías estudiadas. Actualmente existen problemas que no son adecuadamente analizados por métodos deterministas, sin embargo, bien debido al tipo de cargas, bien debido a las características del problema se realiza este tipo de aproximación para obtener resultados aunque sean con una fiabilidad cuestionable. El problema principal elegido es la pérdida de pala de un avión turbohélice por su carácter altamente dinámico y no-lineal. La pérdida de pala de la hélice puede llevar a un fallo catastrófico de la estructura y su simulación requiere un gran esfuerzo de cálculo y de detalle.

A lo largo de la tesis se han revisado los métodos más extendidos en busca de sus limitaciones para proponer alternativas o complementos para el estudio de los problemas. Con este objetivo se van a evaluar los métodos deterministas que son los más extendidos en el campo del cálculo estructural, y en particular los utilizados con el método de los elementos finitos (FEM). Se estudian los métodos explícitos e implícitos de resolución de ecuaciones. Asimismo se reflexiona sobre la forma de analizar problemas complejos con no-linealidades. Para ello se proponen y analizan ejemplos para introducir las distintas herramientas que luego sirven para analizar problemas más complejos. En cada caso se buscan las mejores opciones de modelización para la resolución del problema. Se culmina este proceso de incremento de la dificultad con el estudio determinista de la pérdida de pala.

Finalmente se da un paso más y se ataca el problema con variables aleatorias debido a que existen incertidumbres o propiedades que no pueden reproducirse adecuadamente con variables deterministas. La forma de aproximación al problema es mediante la técnica de Monte Carlo, técnica cuyos costes de cálculo pueden llegar a ser inaceptables. Por este motivo se realizan una serie de optimizaciones. Estos modelos necesitan una formulación que contenga las características completas del sistema para poder realizar un estudio global del problema que incluya las distintas configuraciones del sistema. 
La metodología utilizada en la tesis se podría resumir en:

- Generalización de procedimientos deterministas robustos como el método de elementos finitos para la simulación de distintos fenómenos dinámicos, no lineales incluyendo daños estructurales.

- Desarrollo de códigos de cálculo estructural y disponibilidad de herramientas contrastadas en la simulación de problemas estructurales. Utilización, según problema a abordar, de esquemas tanto implícitos como explícitos para la resolución del problema estructural.

- Formulación del problema estructural con variables gaussianas y no-gaussianas. Desarrollo de los métodos, describiendo, simulando y discretizando el campo gaussiana o no-gaussiano aleatorio. Simulación y estudio de los fenómenos con parámetros con incertidumbres.

\subsection{Descripción de la tesis}

Además de la presente introducción, la tesis se divide en cinco capítulos principales en los que se desarrollan los contenidos introducidos en el apartado anterior, unas conclusiones y unos apéndices útiles para la comprensión completa del contenido.

En el capítulo 2 se introduce brevemente la teoría de los sistemas dinámicos y se plantean algunas de las técnicas y herramientas que luego se van a utilizar a lo largo de los siguientes capítulos.

En el capítulo 3 se describen características y particularidades de problemas dinámicos. Se incluyen ejemplos donde se resuelven las dificultades particulares introducidas por los fenómenos, además, se explica la solución elegida para sus resoluciones. Se hace un repaso de problemas dinámicos altamente no lineales como son los choques, impactos y cargas desequilibrantes que incluyen daños y fallos de las estructuras como es el caso de la frangibilidad. Finalmente se introduce el problema tratado más en profundidad en los siguientes capítulos, la pérdida de pala de un turbohélice.

Para este fenómeno se están desarrollando métodos para la predicción de la respuesta no lineal transitoria de un sistema motor de aeronave debido a la pérdida de una pala, éste es el caso de los próximos capítulos así como de los artículos publicados, ver A.1. La respuesta de la estructura después de una pérdida de pala de la hélice depende radicalmente de la condición de vuelo, la frecuencia de la hélice, el tamaño de la pala perdida, la posición angular donde la pala se pierde y cómo se pierde. Además, la flexibilidad del ala, la rigidez de esta estructura, y el amortiguamiento estructural son también factores determinantes del fenómeno. En el capítulo 4 se realiza un estudio exhaustivo a este respecto desde un punto determinista. Sin embargo, como se determina más adelante en el capítulo 5 la asunción de valores deterministas para determinados parámetros es difícil de mantener. Algunas partes del sistema de unión del motor (especialmente los dispositivos elastoméricos) durante la pérdida de pala, y las zonas y valores 
de fallo a los que se producen están sujetos a las fluctuaciones no deterministas. Resulta de especial interés atacar el problema desde un punto de vista estocástico que lleva a resultados más realistas. Sin embargo; el gran número de variables relevantes hace que sea casi imposible hacer un estudio detallado de todo el sistema. Con el fin de reducir el número de variables en este estudio, se ha realizado un modelo más simplificado que mantiene el comportamiento cualitativo global del sistema. También se ha llevado a cabo una serie de simulaciones Monte Carlo para realizar un análisis paramétrico exhaustivo con variables aleatorias y analizar en detalle la secuencia de rotura elastomérica.

Por lo tanto, en el capítulo 4 la discusión se centra en la resolución por métodos deterministas del problema principal que se ha planteado en esta tesis, la pérdida de pala. Durante su resolución se indican ventajas e inconvenientes de la resolución en particular de este problema. En el capítulo 5 se aproxima el mismo fenómeno pero esta vez mediante métodos aleatorios y, al igual que en el caso anterior, se trata de identificar las limitaciones y bondades del método utilizado.

Finalmente en el capítulo 6 se plantea una discusión final y unas conclusiones junto con unas líneas de investigación que se podían plantear a raíz de este trabajo. Completan la tesis unos apéndices A y B que tratan de ayudar a la comprensión del trabajo presentado. 


\section{ANÁLISIS DE SISTEMAS DINÁMICOS}

\section{Índice}

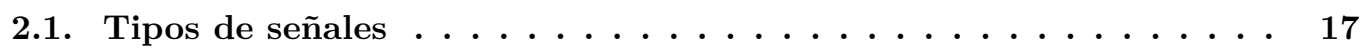

2.1.1. Señales deterministas $\ldots \ldots \ldots \ldots \ldots$

2.1.2. Señales aleatorias . . . . . . . . . . . . . . . . . . . 18

2.1.2.1. Modelos de distribuciones de probabilidad para señales continuas 19

2.2. Teoría de vibraciones . . . . . . . . . . . . . 20

2.2.1. Tipos de sistemas . . . . . . . . . . . . . . 20

2.2.2. Tipo de vibraciones . . . . . . . . . . . . . . . . 21

2.2.2.1. Vibraciones libres . . . . . . . . . . . . . . . 22

2.2.2.2. Vibraciones libres amortiguadas . . . . . . . . . . 22

2.2.2.3. Vibraciones forzadas . . . . . . . . . . . . . . . 23

2.2.2.4. Vibraciones forzadas amortiguadas . . . . . . . . . . 23

2.3. Métodos de integración . . . . . . . . . . . . . . . . 24

2.3.1. Métodos implícitos y explícitos . . . . . . . . . . . . . . 25

2.3.1.1. Métodos de integración . . . . . . . . . . . . . . 27

2.3.1.2. Condición de estabilidad . . . . . . . . . . . . 28

2.3.2. Ejemplo: Sistema de 2 grados de libertad . . . . . . . . . . . . . 28

2.3.2.1. Resolución mediante distintos métodos . . . . . . . . . . . 29

2.3.2.2. Cálculo de modos propios mediante barrido . . . . . . . . . 32

2.4. Método de Craig-Bampton . . . . . . . . . . . . . . . 34

\subsection{Tipos de señales}

Es muy importante entender los principios del análisis de las señales para entender el análisis dinámico de las estructuras. En las estructuras se pueden medir muchos tipos de señales: movimientos (desplazamientos, velocidades, aceleraciones,... ) , cargas aplicadas (fuerzas, presiones,...) u otro tipo de variables físicas como funciones del tiempo. En la realidad estas señales son continuas, sin embargo, las señales analógicas se suelen discretizar para utilizar un 
análisis mediante cálculos discretizados. Esto es lo que sucede mediante el análisis por elementos finitos. Hay que ser conscientes que al discretizar se introduce un error. Existen muchas maneras de definir una señal: se puede hacer en el dominio del tiempo $u=f(t)$ se puede hacer en el dominio de la frecuencia $u=U(\omega)$ o en el espacio $u=\phi(x, y, z)$. Las señales dinámicas pueden ser también deterministas o aleatorias. Las señales deterministas se aplicarían a la ecuación 2.3 directamente, obteniéndose una función matemática sencilla y, en consecuencia, resolviéndose el sistema. Las señales aleatorias no se pueden aplicar de esta manera y hay que recurrir a propiedades estadísticas.

\subsubsection{Señales deterministas}

Las señales deterministas se pueden clasificar como periódicas o no periódicas, ver figura 1.2. Las señales periódicas son aquellas que se repiten en el tiempo:

$$
y(t)=y(t+n T) \text { siendo } \mathrm{n}=1,2,3, \ldots \mathrm{y} \mathrm{T} \text { el período }
$$

Cualquier señal periódica se puede representar como suma infinita de senos y cosenos. Las señales no periódicas no se repiten en el tiempo. Pero esta nueva categoría la podemos subdividir en señales transitorias y señales infinitamente no periódicas. Las variables transitorias son las que no cambian en períodos de tiempo largos pero sí tienen pequeños períodos de gran actividad. Para que una señal sea teóricamente transitoria debería tener un valor constante antes y después del suceso. A efectos prácticos valdrá con que se recoja la señal completamente en el período de tiempo que se considere. Las señales infinitamente no periódicas son aquellas que son continuas pero que no se repiten a lo largo del tiempo. Desde un punto de vista teórico estas señales son señales periódicas con un período infinito.

\subsubsection{Señales aleatorias}

Las señales aleatorias no se pueden predecir y se deben tratar por medio de funciones de probabilidad y variables estadísticas. De forma análoga a las señales deterministas las señales aleatorias se dividen en estacionarias y no estacionarias, ver figura 1.2. Las variables no estacionarias se pueden dividir a su vez en subgrupos, son señales muy difíciles de predecir y de tratar. El índice de la bolsa es un ejemplo de proceso aleatorio de tipo no estacionario aunque en este punto y para este caso caben muchas disquisiciones que se quedarían fuera del ámbito de la tesis. En el caso de las variables estacionarias se pueden subdividir a su vez en ergódicas y no ergódicas. Las señales ergódicas son aquellas en las que las propiedades estadísticas sobre un intervalo temporal son las mismas que sobre toda la señal. En la sección 5.1.1 se introduce un ejemplo con este tipo de señales. 


\subsubsection{Modelos de distribuciones de probabilidad para señales continuas}

En este punto se va a hacer un pequeño inciso para determinar un modelo de distribución para una señal que se encuentra en los fenómenos a estudiar. Sin embargo, se debe tener en cuenta que en la vida real nadie conoce cuál es la auténtica distribución de probabilidad de una variable aleatoria, porque nadie sabe a priori cuál es la función de dicha variable. Todo lo más, se puede calcular la función de modo empírico a partir de los datos de una muestra. Aun así, llegará el momento de pasar al límite, es decir, de inducir una fórmula teórica que corresponda a la distribución de probabilidad que se propone y que se parezca a la distribución empírica de los datos de la muestra. Para ayudar a ese paso al límite, en estadística se estudian modelos teóricos de distribuciones de probabilidad. Se trata de fórmulas teóricas de funciones que pueden resultar adecuadas para determinadas variables aleatorias.

Se van a describir a continuación los modelos de distribuciones de probabilidad más usuales para variables continuas.

- Distribución uniforme: Se dice que una variable aleatoria continua X que sólo puede tomar valores en el intervalo $\left(x_{1}, x_{2}\right)$ sigue una distribución uniforme entre $x_{1}$ y $x_{2}$ si su función de densidad es $f(x)=\frac{1}{\left(x_{2}-x_{1}\right)}$ si $x_{1}<x<x_{2}$ y $f(x)=0$ para los demás valores. Esta función se utiliza cuando se puede escoger cualquier valor del rango con la misma probabilidad.

- Distribución exponencial: Esta distribución suele ser modelo de aquellos fenómenos aleatorios que miden el tiempo que transcurre entre que ocurren dos sucesos. Sea X una variable aleatoria continua que puede tomar valores $x \geq 0$. Se dice que $\mathrm{X}$ sigue una distribución exponencial de parámetro $\gamma$ si su función de densidad $f(x)=\gamma e^{-\gamma x}$ si $x \geq 0$ y $f(x)=0$ para los demás valores. Una variable aleatoria discreta que cuenta el número de éxitos en un determinado período de tiempo. En ese caso, el tiempo que pasa entre dos éxitos consecutivos, T, es una variable aleatoria que sigue una distribución exponencial.

- Distribución Gamma: Sea X una variable aleatoria continua que puede tomar valores $x \geq 0$. Se dice que X sigue una distribución Gamma de parámetros a y $\gamma$ si su función de densidad es $f(x)=\frac{\gamma(\gamma x)^{a-1} e^{-\gamma x}}{\Gamma(a)} u(x)$ donde $\Gamma(x)$ es la función gamma. Sean $X_{1}, \ldots, X_{n}$ variables aleatorias independientes con distribución exponencial. En ese caso, $X=\sum_{i=1}^{n} X_{i}$ sigue una $\operatorname{Gamma}(n, \gamma)$.

- Distribución normal: Sea X una variable aleatoria continua que puede tomar cualquier valor real. Se dice que X sigue una distribución normal o gaussiana, de parámetros $\mu$ y $\sigma$ si su función de densidad es $f(x)=\frac{1}{\sqrt{2 \pi \sigma^{2}}} e^{-\frac{(x-\mu)^{2}}{2 \sigma^{2}}}$ para todo x. El propio nombre de la distribución normal indica su frecuente uso en cualquier ámbito científico y tecnológico. Este uso tan extendido se justifica por la frecuencia o normalidad con la que ciertos fenómenos tienden a parecerse en su comportamiento a esta distribución. Esto, a su vez, es 
debido a que hay muchas variables asociadas a fenómenos naturales cuyas características son compatibles con el modelo aleatorio que supone el modelo de la normal:

- Caracteres morfológicos de individuos (personas, animales, plantas ... ) de una especie (altura, pesos ....).

- Caracteres sociológicos (consumo de cierto producto por un mismo grupo de individuos, puntuaciones de examen...).

- Caracteres psicológicos (cociente intelectual, grado de adaptación a un medio,... ).

- Errores cometidos al medir ciertas magnitudes.

- Valores estadísticos muestrales, como por ejemplo la media.

- Otras distribuciones como la binomial o la de Poisson son aproximadas por la normal, $\ldots$

En general cualquier característica que se obtenga como suma de muchos factores independientes encuentra en la distribución normal un modelo adecuado. El teorema central del límite dice sean $X_{1}, \ldots, X_{N}$ variables aleatorias independientes, todas ellas con la misma distribución de probabilidad, distribución de media $\mu_{x}$ y desviación típica $\sigma_{x}$. En ese caso, la suma de estas variables sigue aproximadamente una distribución normal cuando $\mathrm{N}$ es elevado, es decir, $\sum_{i=1}^{n} X_{i} \approx N\left(N \mu_{x}, \sqrt{N} \sigma_{x}\right)$. Despejando y cambiando de variable, el Teorema Central del Límite igualmente dice $\frac{\sum_{i=1}^{n} X_{i}-N \mu_{x}}{\sqrt{N} \sigma_{x}} \approx N(0,1)$. Por lo tanto, sumar un gran número de sucesos aleatorios elementales se puede modelar mediante la distribución gaussiana y esto es lo que sucede para un gran número de fenómenos reales. Enunciando el Teorema Central del Límite en términos de la media, $\bar{X}$, de las variables $X_{1}, \ldots, X_{N}$, podemos decir que si $\mathrm{N}$ es grande, $\bar{X} \approx N(\mu, \sigma / \sqrt{N})$.

\subsection{Teoría de vibraciones}

Hay que tener en cuenta que no siempre hay que considerar que un fenómeno es dinámico, esto sucede cuando las fuerzas de inercia no son despreciables. A continuación en las próximas secciones, se van a mostrar las ecuaciones fundamentales del movimiento y distintos tipos de movimientos dinámicos.

\subsubsection{Tipos de sistemas}

Los sistemas en la realidad son continuos y sus parámetros no son fijos. Sin embargo, si se dan ciertas condiciones es posible realizar simplificaciones en el sistema y representar el sistema con un número finito de grados de libertad y sus parámetros como parámetros fijos.

El número de desplazamientos desconocidos (o en su defecto velocidades o aceleraciones) se conoce como número de grados de libertad (DOF) del sistema. Un sistema continuo implica 
un número infinito de grados de libertad, en cambio, un sistema puede que se represente correctamente por un número finito de masas en determinados puntos de la estructura. La técnica del método de elementos finitos es la más extendida y utilizada y realiza esta discretización. Matemáticamente esta discretización también simplifica el problema y el comportamiento del sistema discreto con parámetros fijos se puede representar con ecuaciones diferenciales ordinarias cuando parámetros distribuidos necesitarían ecuaciones en derivadas parciales.

El sistema más simple es el sistema de un grado de libertad. A continuación se hace un breve repaso a la teoría de estos sistemas. Las fuerzas que actúan sobre este tipo de sistema son:

- Fuerzas elásticas: $F_{e}=-k x$ donde $k$ es la rigidez del muelle. Se pone un signo menos ya que la fuerza se opone al movimiento.

- Fuerzas amortiguadoras o disipadoras: $F_{d}=-c \dot{x}$, en este caso se muestra el modelo de fuerza viscosa, donde $c$ es el coeficiente de viscosidad. Se opone al movimiento.

- Fuerza excitadora: $F_{\text {ext }}$ es la fuerza exterior que excita el sistema.

Por lo tanto se tiene la siguiente ecuación:

$$
m \ddot{x}=-k x-c \dot{x}+F_{\text {ext }}
$$

donde $\mathrm{m}$ es la masa del sistema. Este sistema se refiere a un grado de libertad traslacional pero podría tratarse de un giro. Entonces:

- Momento elástico: $M_{e}=-k \theta$

- Momento amortiguador o disipador: $M_{d}=-c \dot{\theta}$

- Momento excitador: $M_{e x t}$

En este caso:

$$
J \ddot{\theta}=-k \theta-c \dot{\theta}+M_{e x t}
$$

donde $\mathrm{J}$ es el momento de inercia.

\subsubsection{Tipo de vibraciones}

Las vibraciones pueden ser libres o forzadas. Las vibraciones libres ocurren cuando no hay fuerzas externas y el sistema oscila debido a las propias fuerzas internas. Las frecuencias naturales del sistema están definidas por la masa y elasticidad del sistema, estas frecuencias son fundamentales para conocer el movimiento. Las vibraciones forzadas se dan cuando existe una fuerza externa o excitación que actúa sobre el sistema. Otro parámetro importante en estos sistemas es el amortiguamiento ya que supondrá que el movimiento del sistema pierda o no energía. 
En las siguientes secciones se van a mostrar las ecuaciones de distintos tipos de movimientos para uno o varios grados de libertad. Estos movimientos son los que tienen cualquier elemento dentro de un método de los elementos finitos para un incremento de tiempo.

\subsubsection{Vibraciones libres}

En este tipo de vibraciones no hay fuerza excitadora.

$$
m \ddot{x}+k x=0
$$

Esta ecuación se puede poner de la forma:

$$
\ddot{x}+\omega^{2} x=0
$$

donde $\omega=\sqrt{\frac{k}{m}}$ es la frecuencia angular propia del sistema. La respuesta en este tipo de problemas es de la forma.

$$
x=A \sin (\omega t)+B \cos (\omega t)
$$

donde A y B se determinan con las condiciones iniciales del problema. En el caso de ser más grados de libertad la ecuación se quedaría:

$$
x=A \sin \left(\omega_{n} t\right)+B \cos \left(\omega_{n} t\right)
$$

con $\omega_{n}=\sqrt{\frac{k_{n}}{m_{n}}}$ de cada grado de libertad. También se puede indicar de la forma:

$$
x=C \sin \left(\omega_{n} t+\phi\right)
$$

donde $\mathrm{C}$ y $\phi$ también se determinan de igual manera. Además $C=\sqrt{A^{2}+B^{2}}$ y $\phi=\tan ^{-1} \frac{B}{A}$.

\subsubsection{Vibraciones libres amortiguadas}

En este tipo de vibraciones tampoco hay fuerza excitadora pero este caso se tiene en cuenta el término amortiguador. Este término puede ser viscoso $-c \dot{x}$, constante cte (modelo de la ley de Coulomb mediante el coeficiente de fricción) o seguir una ley arbitraria $-c \dot{x}^{n}(n>1)$. Las soluciones del desplazamiento para el caso viscoso son las siguientes:

$$
\begin{aligned}
& x=e^{\lambda t} \\
& \lambda_{1}=-\frac{c}{2 m}+\sqrt{\left(\frac{c}{2 m}\right)^{2}-\frac{k}{m}} \\
& \lambda_{1}=-\frac{c}{2 m}-\sqrt{\left(\frac{c}{2 m}\right)^{2}-\frac{k}{m}} \\
& c_{c}=2 m \sqrt{\frac{k}{m}}=2 m \omega_{n}
\end{aligned}
$$

donde $c_{c}$ es el amortiguamiento crítico según el cual el movimiento oscila alrededor de la solución estática o se dirige directamente hacia ella. 


\subsubsection{Vibraciones forzadas}

En este tipo de vibraciones hay fuerza excitadora. Si suponemos que la fuerza es periódica $F=F_{0} \sin \left(\omega_{0} t\right)$ donde $F_{0}$ es la amplitud de la fuerza y $\omega_{0}$ su frecuencia. En este caso:

$$
m \ddot{x}=-k x+F_{0} \sin \left(\omega_{0} t\right)
$$

En este caso la solución se compone de una solución particular $x_{p}$ más la solución complementaria, la del problema libre, $x_{c}$.

$$
\begin{aligned}
& x=x_{c}+x_{p} \\
& x_{c}=C \sin \left(\omega_{n} t+\phi\right) \\
& x_{p}=\frac{\frac{F_{0}}{k}}{1-\left(\frac{\omega_{0}}{\omega_{n}}\right)^{2}} \sin \left(\omega_{0} t\right)
\end{aligned}
$$

\subsubsection{Vibraciones forzadas amortiguadas}

Finalmente si se tienen en cuenta todos los términos a la vez:

$$
m \ddot{x}=-k x-c \dot{x}+F_{0} \sin \left(\omega_{0} t\right)
$$

En este caso vuelve a haber la suma de dos soluciones $x=x_{c}+x_{p}$ la solución complementaria es la mostrada en 2.2.2.2, la solución particular es de la forma:

$$
\begin{aligned}
& x=x_{c}+x_{p} \\
& x_{c}=C \sin \left(\omega_{n} t+\phi\right) \\
& x_{p}=X^{\prime} \sin \left(\omega_{0} t-\phi^{\prime}\right) \\
& X^{\prime}=\frac{\frac{F_{0}}{k}}{\sqrt{\left.\left[1-\left(\frac{\omega_{0}}{\omega_{n}}\right)^{2}\right]^{2}+2\left(\frac{c}{c_{c}}\right)\left(\frac{\omega_{0}}{\omega_{n}}\right)\right]^{2}}} \\
& \phi^{\prime}=\tan ^{-1}\left[\frac{2\left(\frac{c}{c_{c}}\right)\left(\frac{\omega_{0}}{\omega_{n}}\right)}{1-\left(\frac{\omega_{0}}{\omega_{n}}\right)^{2}}\right]
\end{aligned}
$$

Dependiendo de la amplitudes y frecuencias de la fuerza excitadora, de las frecuencias naturales y amortiguaciones del sistema sucede que la respuesta se amortigua o crece en amplitud con el tiempo pudiendo llegar a un fallo crítico en el sistema.

En este punto aparece el término del factor de amplificación dinámica máximo:

$$
D=\frac{X^{\prime}}{\frac{F_{0}}{k}}
$$

Donde además $\xi=\frac{c}{c_{c}}$ y $\beta=\frac{\omega_{0}}{\omega_{n}}$. Se cumple que el factor de amplificación dinámica máximo para cada valor de $\xi$ viene dado por:

$$
D_{\max }=\frac{1}{1 \xi \sqrt{1-2 \xi^{2}}}
$$

$\mathrm{Y}$ se da para un valor de $\beta_{\max }=\sqrt{1-2 \xi^{2}}$. Se observa que para $\beta=0$, lo que supone una carga de frecuencia nula y, por lo tanto, estática, la respuesta dinámica coincide efectivamente 
con la estática $(\mathrm{D}=1)$. Por otra parte, cuando $\beta \approx 1$, lo que significa que la frecuencia de la carga coincide con la natural del sistema, se produce el fenómeno de la resonancia, consistente en que la respuesta dinámica llega a ser mucho mayor que la estática al producirse un proceso de realimentación que va haciendo crecer la respuesta. Si bien el máximo de la curvas no está exactamente en $\beta=1$, se acerca a este punto a medida que decrece la relación de amortiguamiento $\xi$. Por último, para valores altos de $\beta(\beta=3$ ó $\beta=4)$ la respuesta dinámica es menor que la estática, debido a que la carga oscila tan rápidamente que el sistema no es capaz de seguirla y, por lo tanto, apenas se mueve.

\subsection{Métodos de integración}

La ecuación del movimiento es:

$$
M y^{\prime \prime}+C y^{\prime}+K y=F
$$

Donde $\mathrm{M}=$ Matriz másica, $\mathrm{C}=$ Matriz de amortiguamiento, $\mathrm{K}=$ Matriz elástica y $\mathrm{F}=$ Fuerzas exteriores. En un problema no lineal la ecuación 2.3 tiene que resolverse por iteraciones. El término másico suele ser lineal. Sin embargo, los términos elástico y de amortiguamiento pueden ser no lineales. Cuando todos los términos son lineales se suele utilizar la superposición modal para su resolución. Muchas veces no es necesario la utilización de innumerables modos sino los necesarios para que contengan la mayoría del comportamiento estructural. Sin embargo, es necesario un conocimiento del modelo muy profundo para conocer los modos y sus frecuencias. De todas maneras, si se tienen en cuenta estos modos se conoce el comportamiento de la estructura, por lo tanto, en un análisis por elementos finitos es un paso ineludible y no se pasa a analizar dinámicamente sin llevar a cabo un análisis estático lineal y un chequeo de modos.

Sin embargo, si el problema es no lineal bien por las condiciones de contorno, bien por las ecuaciones constitutivas (no linealidad de material, contactos, etc.) o bien por que se produzcan grandes desplazamientos, entonces, el análisis es mucho más complejo. Cuando hay términos no lineales no se puede llevar a cabo esta superposición ya que los modos cambian durante el análisis y se suele utilizar una integración directa en el tiempo. Tampoco se suele utilizar la superposición modal cuando el espectro de las fuerzas exteriores es complejo ya que implicaría la utilización de muchos modos y penaliza la resolución del análisis por este método.

Cuando el término elástico y/o el de amortiguamiento son no lineales se resuelve la ecuación mediante iteraciones que linealizan cada paso. En este trabajo la forma más común en las que se van a encontrar los términos es la siguiente:

$$
M y^{\prime \prime}+C y^{\prime}+K y=F \rightarrow M y^{\prime \prime}(t)+C y^{\prime}(t)+K y(t)=F_{\text {nolineal }}(y, t)
$$

Por lo tanto cuando se obtiene la solución se utilizan muchos pasos de tiempo, a veces son necesarios tantos que hace inabordable la búsqueda de la solución por este método. Esto sucede 
cuando el modelo es grande, entonces, se debe tratar de buscar una simplificación o reducción del problema.

Finalmente, se podrían hacer las siguientes observaciones. El caso lineal converge, en cambio, en el caso no lineal la rigidez de la estructura suele decrecer para la mayoría de los problemas y lo mismo suele suceder con las frecuencias propias. Por consiguiente, los algoritmos estables (que son los algoritmos que en ausencia de carga exterior mantienen la solución entre unos límites) para las propiedades iniciales de material suelen mantenerse estables a lo largo de todo el análisis. Lo mismo sucede con la precisión de la solución. Sin embargo en el caso de que la rigidez aumente (es el caso de contactos o dispositivos elastoméricos con propiedades hiperelásticas). Entonces, los algoritmos se vuelven inestables. En estos casos los parámetros y el paso de tiempo debe seleccionarse teniendo en cuenta la rigidez más alta.

\subsubsection{Métodos implícitos y explícitos}

La resolución de las ecuaciones puede efectuarse con metodología implícita y explícita. La metodología implícita presenta las siguientes características:

- Incondicionalmente convergente. Independiente del paso de tiempo de integración.

- Su resolución implica resolver el sistema completo. Los algoritmos de computación son más lentos, requiriendo igualmente más memoria y es un algoritmo sensible a fenómenos de inestabilidad elástica por tener que invertir matrices por lo que se hace necesario reducir el paso de integración.

- La precisión en los resultados está basada en las tolerancias impuestas al equilibrio dinámico del sistema.

La metodología explícita presenta las siguientes características:

- Algoritmo robusto y simple de programar.

- Condicionalmente convergente, requiere pasos pequeños de tiempo para la integración.

- Su resolución implica resolver las ecuaciones a nivel de elemento.

- Los algoritmos de computación son rápidos y requieren igualmente poca memoria.

- En los problemas de choque donde se involucra un rango de frecuencias amplio es necesario extender el cálculo a tiempos que son varios órdenes de magnitud superiores a los de aplicación de las cargas.

Exactamente los métodos explícitos de los elementos finitos resuelven las ecuaciones de onda en la zona del frente de onda, en realidad esto no requiere de la resolución de un número grande de ecuaciones. Por el contrario, los códigos implícitos incondicionalmente estables resuelven el 
problema para todo tipo de movimientos y ecuaciones, aunque estén acopladas. El paso de tiempo requerido en estos códigos es por lo general dos o tres órdenes de magnitud mayores que en los códigos implícitos.

En el caso de simulaciones de impactos o choques que implican el uso de contactos y modelos de material no lineales los códigos explícitos se muestran más versátiles y eficientes. Los esquemas explícitos pueden ser más eficaces que los implícitos en aquellas aplicaciones caracterizadas por:

- Tiempos de estudio del fenómeno no muy elevados. Las técnicas implícitas pueden ser más eficaces si no se requiere conocer la evolución si no sólo el estado final.

- No linealidades presentes en el estudio ya que la complejidad de la estructura no afecta al tiempo de análisis usando esquemas explícitos. En los esquemas implícitos el número de matrices a generar en cada paso depende fuertemente del comportamiento no lineal del sistema.

- Discretización espacial requerida en los métodos explícitos el esfuerzo de cálculo varía linealmente con el tamaño del modelo. En las técnicas explícitas el esfuerzo crece exponencialmente con el número de elementos.

Cuando estas características se cumplen, las técnicas implícitas necesitan incrementar considerablemente el número de puntos intermedios de equilibrio, lo que implica disminuir el paso de integración, y reconstruir las matrices globales del sistema. Los casos en cuestión son los que tienen períodos de duración inferiores a 100 ms, cargas discontinuas o fuerzas de inercia significativas (por ejemplo impacto, detonaciones, choques,...). En estos casos, como no es necesario calcular la matriz de rigidez global ni establecer las condiciones de equilibrio, las variaciones en las condiciones de contorno o en las cargas originadas por los contactos se manejan de forma muy eficiente con métodos explícitos. Se utilizan cuando el sistema puede tener movimiento como sólido rígido o cuando los elementos prácticamente no se deforman cuando se les somete a cargas. Es importante resaltar que únicamente algunos códigos implícitos no lineales manejan adecuadamente estos casos. El caso de las bifurcaciones en las estructuras se pueden encontrar en el pandeo, más concretamente en la repuesta en post-pandeo, "snap through", en los cuales la estructura puede comportarse súbitamente como un mecanismo con cambios muy rápidos de rigidez, que en algunos casos se combinan con aparición de posibles contactos. Otros casos particulares son las no linealidades del material como plasticidad o rotura, en estos casos, el material cambia de rigidez en intervalos de tiempo muy pequeños. Por tanto, la necesidad de actualizar continuamente la matriz de rigidez penaliza de forma considerable los métodos explícitos. En estos casos en los que se producen rotura y fragmentaciones la matriz de masa cambia de forma significativa. Esto sucede en choques a alta velocidad y en elementos frangibles. 


\subsubsection{Métodos de integración}

En un método de integración explícito se utilizan técnicas numéricas para integrar un sistema de ecuaciones diferenciales ordinarias y discretizando el espacio continuo. Se denomina explícito debido a que los desplazamientos del paso siguiente vienen dados explícitamente de los anteriores. El equilibrio se aplica en un tiempo determinado donde se conocen todas las posiciones en el espacio. Las aceleraciones se determinan de ese equilibrio. Una técnica de diferencias centradas permite determinar los desplazamientos en el siguiente paso de tiempo y repetir el proceso cuantas veces sea necesario. Este proceso sólo requiere invertir la matriz de masas y si esta matriz fuera diagonal no sería ni necesario. Si se toman las precauciones adecuadas con la integración explícita se puede conseguir un segundo orden de precisión, todo depende de la naturaleza de los elementos ya que es una técnica que va elemento a elemento. El único inconveniente de los algoritmos explícitos son la condición de inestabilidad y la incapacidad que muestran para tratar problemas estáticos.

En el caso de los implícitos los desplazamientos en un paso no se conocen explícitamente del anterior y hay que resolver un sistema de ecuaciones. Esquemas implícitos e incondicionalmente estables (para problemas lineales) como el método de $\beta$-Newmark con $\alpha=0,5$ y $\beta=0,25$ son generalmente escogidos para resolver este tipo de problemas. En particular el método de $\beta$ Newmark utiliza la regla trapezoidal de integración, es de diferencias centradas y linealiza las aceleraciones. La idea fundamental del método para los valores de sus parámetros mencionados es suponer una aceleración constante entre pasos de tiempo. Estos parámetros también hacen al método incondicionalmente estable. Sin embargo, el método implícito que es incondicionalmente estable para problemas lineales puede comenzar a mostrar inestabilidad cuando se utiliza para problemas no lineales, especialmente para simulaciones de tiempo largos. Como se ha comentado el uso de grandes pasos de tiempo no permite la reproducción precisa de los modos más altos. Estos modos, a pesar de que pudieran no contener la parte interesante de la solución pueden contribuir a acumular errores durante mucho tiempo, y en última instancia, afectar a la estabilidad de la solución. Esta es una razón para analizar el problema mediante un método FEM explícito, que reproduce los modos más altos, sin embargo, requiere un gran número de incrementos de tiempo (aunque con un coste computacional relativamente bajo). El modelo FEM utiliza el esquema explícito de MSC.Dytran que es un código de análisis dinámico transitorio. El esquema que utiliza es de diferencias centradas tanto para las velocidades como los desplazamientos. El esquema sigue calcula estas variables una sola vez en cada paso de tiempo.

$$
\begin{gathered}
v_{n+\frac{1}{2}}=v_{n+\frac{1}{2}}+a_{n} \frac{\Delta t_{n+\frac{1}{2}}+\Delta t_{n-\frac{1}{2}}}{2} \\
x_{n+1}=x_{n}+v_{n+\frac{1}{2}} \Delta t_{n+\frac{1}{2}}
\end{gathered}
$$

El uso de métodos implícitos requiere resolver la matriz del sistema. La resolución de la matriz del sistema se realiza una sola vez si el sistema es lineal, sin embargo, esto no es así 
en los casos no lineales. Por lo tanto si el incremento de tiempo es muy pequeño, los cálculos se vuelven inabordables. Esta es una razón principal para analizar el problema a través de un modelo simplificado. Por ejemplo, para el método de Runge-Kutta de orden cuatro, el cálculo de un vector de fuerzas internas se realiza cuatro veces. En este caso puede ser que sea menos costoso que la resolución de la matriz del sistema. Sin embargo, este es el paso del esquema más costoso computacionalmente en métodos explícitos, y puede ser inasumible si el cálculo del vector de las fuerzas internas implica funciones no lineales.

\subsubsection{Condición de estabilidad}

En los métodos explícitos no hay que resolver ningún sistema de ecuaciones y cada paso no consume casi recursos computacionales. Sin embargo, los métodos explícitos no son quizás los más utilizados y esto es por la condición de estabilidad. El incremento de tiempo crítico para un sistema de múltiples grados de libertad está condicionado por el modo a reproducir más alto. Por lo tanto un incremento de tiempo pequeño conllevará soluciones más realistas y estables. Consecuentemente si la mayor parte de la solución está contenida en los modos más bajos es posible utilizar un incremento de tiempo considerablemente mayor que el crítico. El modelo simplificado reduce los modos y sólo tiene en cuenta los más importantes por lo tanto aumenta el incremento de tiempo crítico. La ecuación típica de estabilidad condicional es:

$$
\Delta t \leq \frac{l_{c}}{c}=\frac{2}{\omega_{\max }} \quad \text { y } \quad \omega_{\max } \rightarrow \omega_{0}=\sqrt{\frac{k}{m}}
$$

Los pasos de tiempo vienen determinados por dividir la longitud del elemento característico por la velocidad del sonido en el material del elemento. Por lo tanto, el paso del tiempo del análisis no debe superar el paso de tiempo que marca el elemento más pequeño. Normalmente el paso de tiempo que se escoge es lo más parecido al paso de tiempo crítico. Si la malla es muy fina el paso de tiempo crítico se hará más pequeño. Esto puede hacer que el número de pasos de tiempo pueda ser enorme y haga prohibitivo el análisis. Esta es una de las razones por las que estos métodos sean los preferidos para explosiones, impacto, choques y problemas similares donde el tiempo total a analizar es pequeño y aunque el espectro de frecuencias de las cargas contenga frecuencias altas en otros problemas como terremotos, vibraciones y problemas donde las frecuencias que se consideran son menores y el tiempo a analizar es alto estos métodos se pueden volver ineficientes por la condición de estabilidad.

\subsubsection{Ejemplo: Sistema de 2 grados de libertad}

A continuación se realiza una resolución analítica, y por métodos explícito e implícito de sistemas de 2 grados de libertad. Finalmente también se realiza un cálculo de modos propios mediante un barrido de frecuencias. 


\subsubsection{Resolución mediante distintos métodos}

El problema se muestra en la figura 2.1. Se usa un método explícito de diferencias centrales.

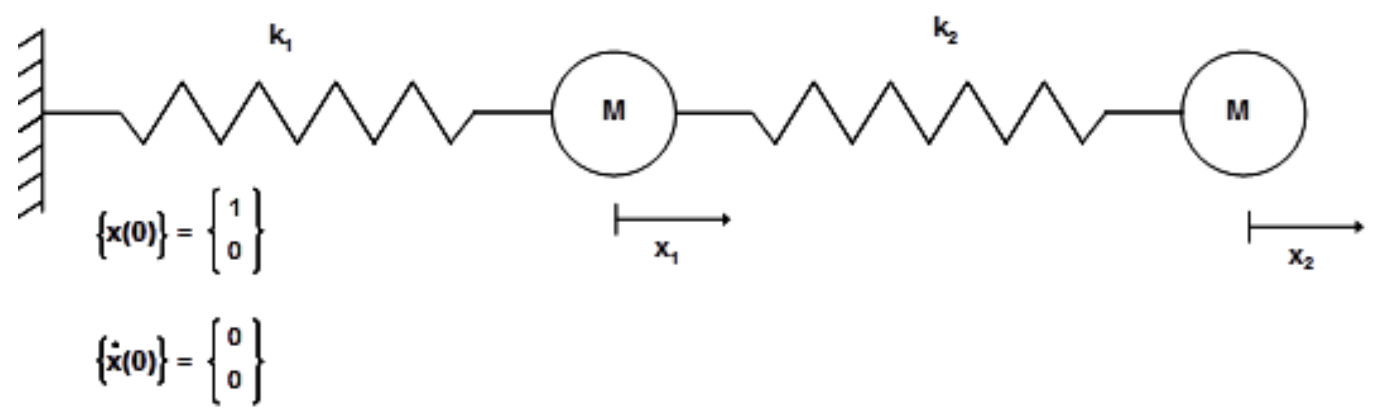

Figura 2.1: Problema de 2 grados de libertad

La expresión general del método es:

$$
[M]\left\{\frac{x_{n+1}-2 x_{n}+x_{n-1}}{\Delta t^{2}}\right\}+[B]\left\{\frac{x_{n+1}-x_{n-1}}{2 \Delta t}\right\}+[K]\left\{x_{n}\right\}=\left\{F_{n}\right\}
$$

$\mathrm{Y}$ con $[\mathrm{B}]$ y $[\mathrm{M}]$ diagonales.

$$
\left\{x_{n+1}\right\}=\left[[M]_{n}+\frac{\Delta t}{2}[B]_{n}\right]^{-1}\left[\Delta t^{2}\left(-[k]_{n}\left\{x_{n}\right\}+\left\{F_{n}\right\}\right)+2[M]_{n}\left\{x_{n}\right\}-\left([M]_{n}+\frac{\Delta t}{2}[B]_{n}\right)\left\{x_{n-1}\right\}\right]
$$

Siendo $x_{-1}=-2 \Delta t \dot{x}(0)+x(0)$. Y como en este caso $[B]=0$ y $\left\{F_{n}\right\}=0$.

$$
\left\{x_{n+1}\right\}=\left[[M]_{n}\right]^{-1}\left[\Delta t^{2}\left(-[k]_{n}\left\{x_{n}\right\}\right)+2[M]_{n}\left\{x_{n}\right\}-\left([M]_{n}\right)\left\{x_{n-1}\right\}\right]
$$

Con $x_{0}=x_{-1}=x(0)$.

$$
x_{n+1}=M^{-1}\left[-k \Delta t^{2} x_{n}+2 M x_{n}-M x_{n-1}\right]
$$

En cuanto al método implícito se sigue un esquema numérico de $\beta$-Newmark. Como $[B]=0$ y $\left\{F_{n}\right\}=0$ y $\beta=0,35$ y $\gamma=0,5$, quedaría:

$$
\begin{aligned}
& \alpha_{0}=\frac{1}{0,35 \Delta t^{2}}, \alpha_{1}=\frac{1}{0,7 \Delta t}, \alpha_{2}=\frac{1}{0,35 \Delta t}, \alpha_{3}=\alpha_{4}=\frac{3}{7}, \alpha_{5}=-\frac{2 \Delta t}{7}, \alpha_{6}=\alpha_{7}=\frac{\Delta t}{2} \\
& x_{n+1}=\left(\frac{M}{0,35 \Delta t^{2}}+k\right)^{-1}\left(\frac{M}{0,35 \Delta t^{2}} x_{n}+\frac{M}{0,35 \Delta t} \dot{x}_{n}+\frac{3}{7} M \ddot{x}_{n}\right) \\
& \dot{x}_{n+1}=\frac{1}{0,7 \Delta t}\left(x_{n+1}-x_{n}\right)-\frac{3}{7} \dot{x}_{n}+\frac{2 \Delta t}{7} \ddot{x}_{n} \\
& \ddot{x}_{n+1}=\frac{1}{0,35 \Delta t^{2}}\left(x_{n+1}-x_{n}\right)-\frac{1}{0,35 \Delta t} \dot{x}_{n}+\frac{3}{7} \ddot{x}_{n} \\
& x_{0}=x_{-1}=x(0) \\
& \dot{x}_{0}=\dot{x}_{-1}=\dot{x}(0)=0 \\
& \ddot{x}_{0}=\ddot{x}_{-1}=\ddot{x}(0)=0
\end{aligned}
$$

Resolviendo el problema analíticamente mediante las ecuaciones 2.2.2.1.

$$
\begin{aligned}
& x_{1}=\frac{1}{2}\left(\cos \left(\omega_{1} t\right)+\cos \left(\omega_{2} t\right)\right)+\frac{\sqrt{5}}{10}\left(\cos \left(\omega_{1} t\right)+\cos \left(\omega_{2} t\right)\right) \\
& x_{2}=-\frac{\sqrt{5}}{5}\left(\cos \left(\omega_{1} t\right)+\cos \left(\omega_{2} t\right)\right)
\end{aligned}
$$


Con $\omega_{1} \mathrm{y} \omega_{2}$.

$$
\begin{aligned}
\omega_{1} & =\sqrt{\frac{1}{2} \frac{k}{M}(3+\sqrt{5})} \\
\omega_{2} & =\sqrt{\frac{1}{2} \frac{k}{M}(3-\sqrt{5})}
\end{aligned}
$$

En todos los métodos de resolución $[M]=\left[\begin{array}{cc}M & 0 \\ 0 & M\end{array}\right]$ y $[K]=\left[\begin{array}{cc}2 k & -k \\ -k & k\end{array}\right]$. A continuación se presentan las gráficas obtenidas para cada uno de los métodos y los tiempos de proceso con cada uno de los métodos. La figura 2.2 muestra el tiempo de cálculo en función del paso de tiempo utilizado, para incrementos de tiempo bajos el solver explícito es más rápido que el implícito, lo contrario sucede para incrementos de tiempo altos.

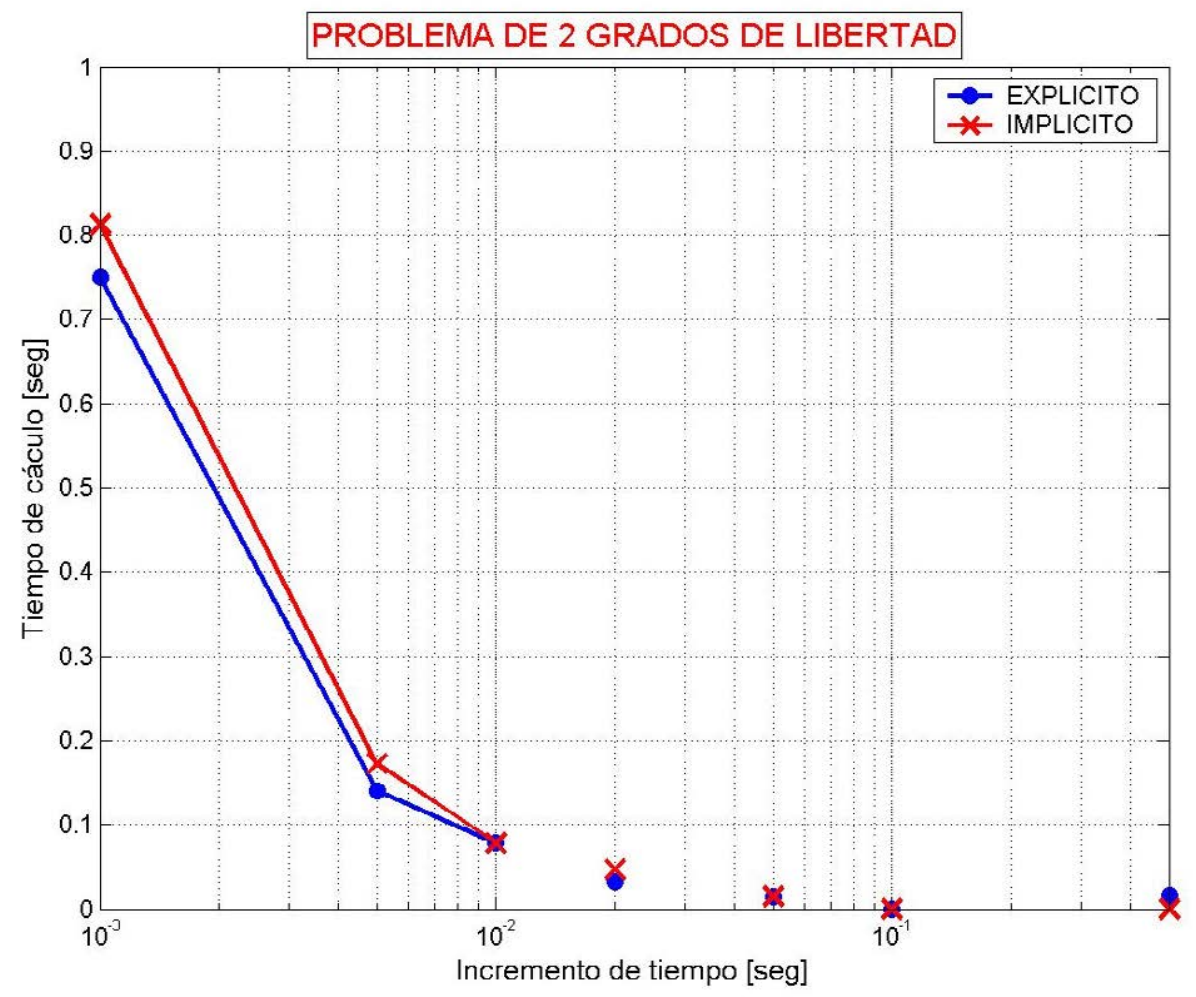

Figura 2.2: Tiempo de cálculo vs. incremento de tiempo en cada paso

La figura 2.3 muestra los resultados del método explícito en función del paso de tiempo utilizado, se muestra como para valores mayores a 0.05 el resultado de la simulación se aleja de la solución analítica. En este caso la simulación tiende a adelantarse, más cuanto mayor sea el paso de tiempo.

La figura 2.4 los resultados del implícito en función del paso de tiempo utilizado, se muestra como para valores mayores a 0.05 el resultado de la simulación se aleja de la solución analítica. En este caso la simulación tiende a atrasarse, más cuanto mayor sea el paso de tiempo. 


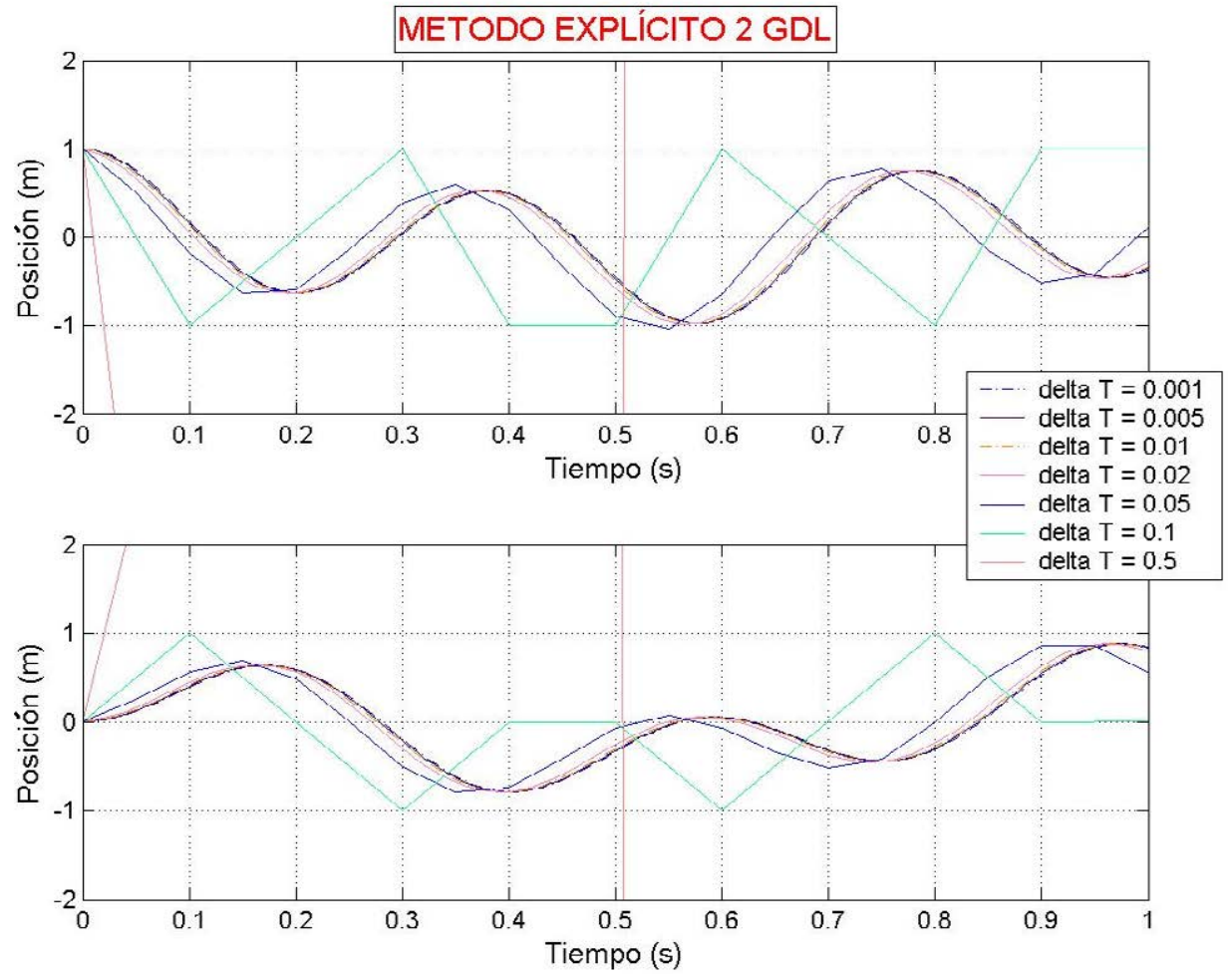

Figura 2.3: Resultados del nodo 1 (arriba) y nodo 2 (abajo) del método explícito

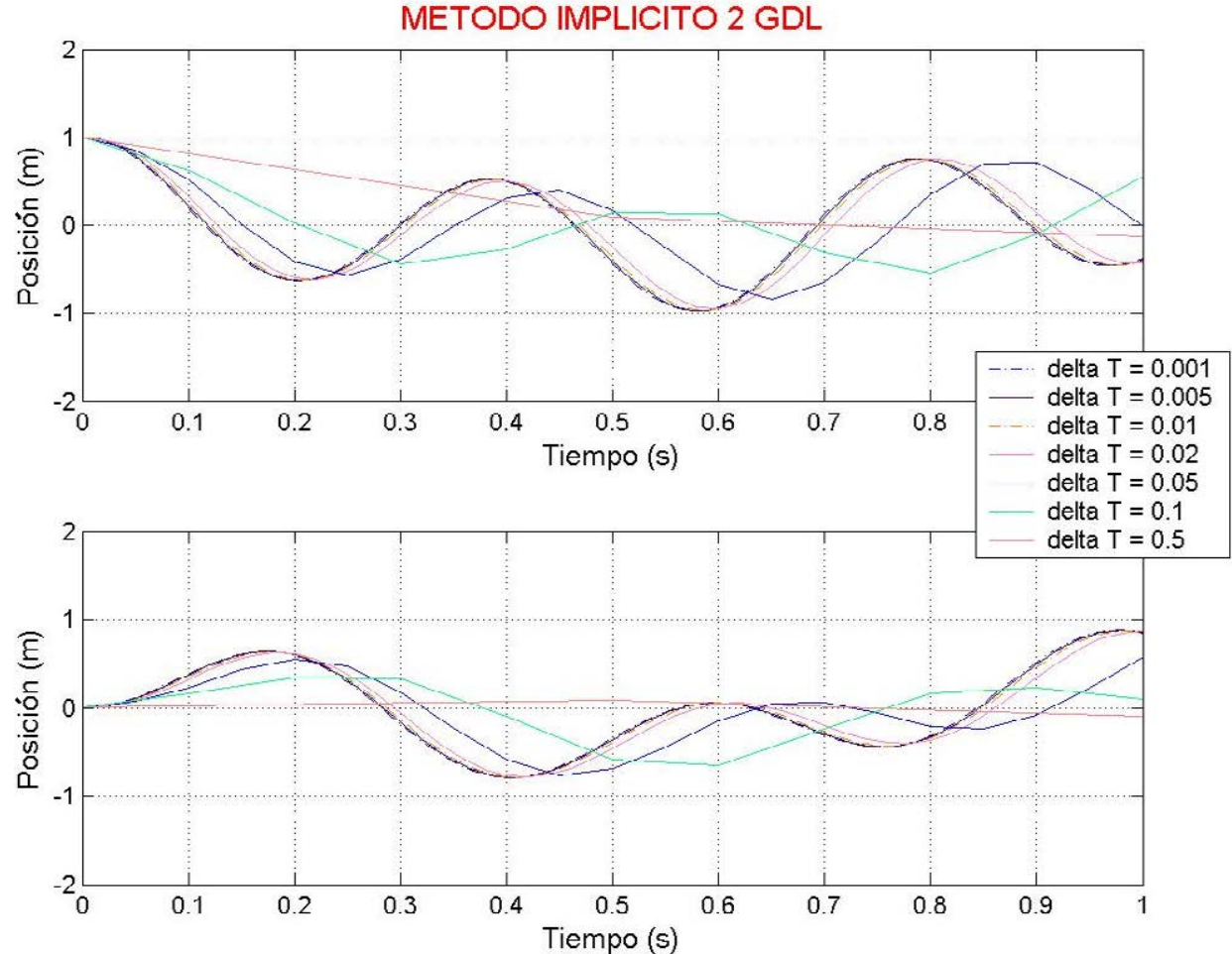

Figura 2.4: Resultados del nodo 1 (arriba) y nodo 2 (abajo) del método implícito 


\subsubsection{Cálculo de modos propios mediante barrido}

Para este caso se harán las 2 masas $\mathrm{M}=1 \mathrm{~kg}$ y los dos muelles $\mathrm{K}=100 \mathrm{~N} / \mathrm{m}$. Las condiciones iniciales son nulas, la fuerza excitadora tiene una amplitud de $0.1 \mathrm{~N}$, y el tamaño de paso en el método explícito es de $0.005 \mathrm{~s}$. El método para obtener las frecuencias propias es hacer un barrido de frecuencias. Se ha elegido desde 0 a $20 \mathrm{rad} / \mathrm{s}$. En la figura 2.5 se puede ver la respuesta del sistema a este barrido de frecuencia, donde se observan claramente dos máximos que corresponden a los modos propios (el valor observado es el máximo desplazamiento en valores absolutos de cada nodo obtenido en un tiempo de 10s). Estos máximos coinciden con las frecuencias analíticas calculadas de las ecuaciones 2.3.2.1, $\omega_{1}=6,18 \mathrm{rad} / \mathrm{s} \mathrm{y} \omega_{2}=16,18$. Una vez

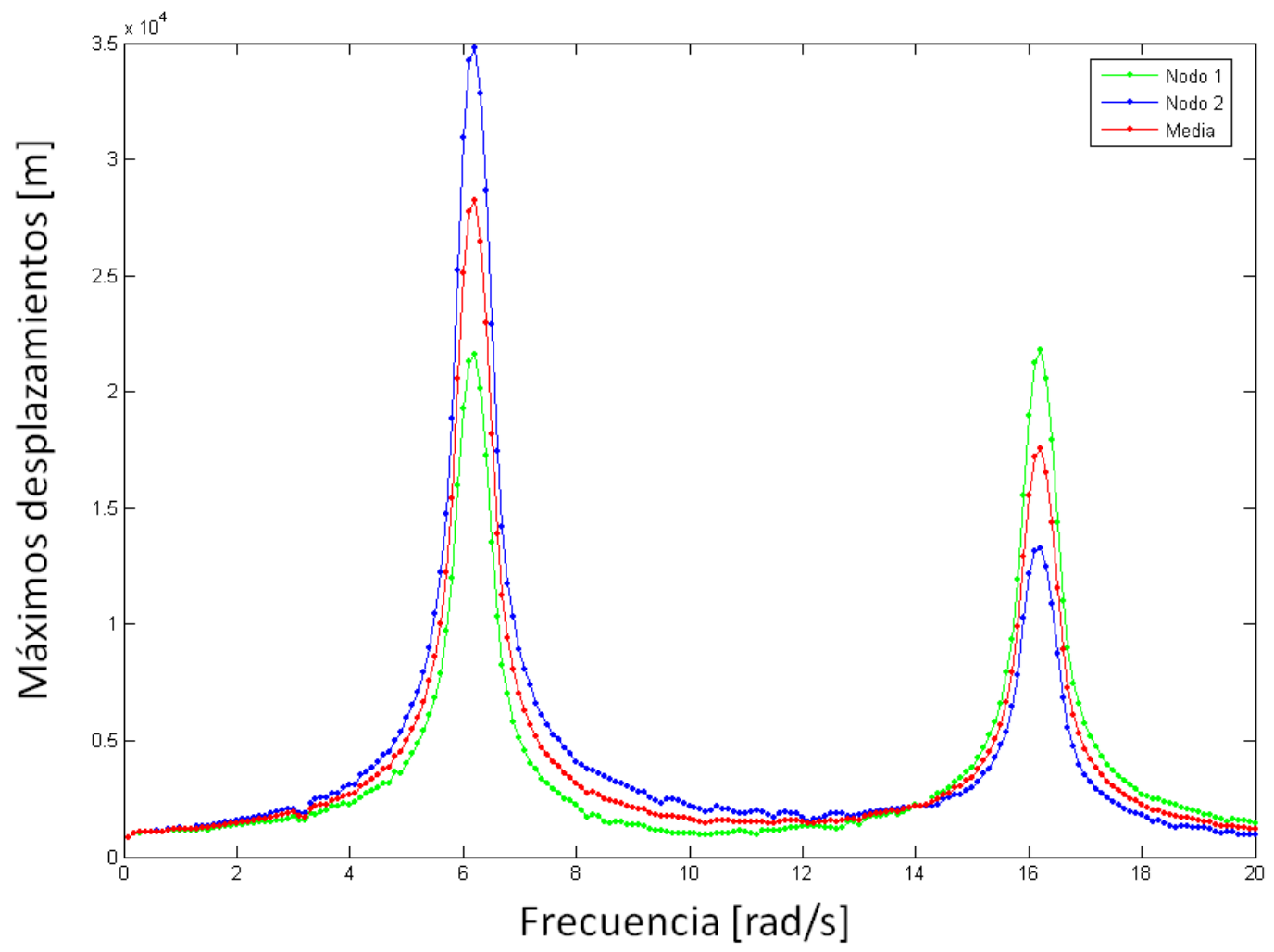

Figura 2.5: Desplazamientos en función de la frecuencia

estimadas las frecuencias vemos los modos. Para ello se observan los máximos desplazamientos en la respuesta del sistema para esas frecuencias. Como éste es un sistema sin amortiguamiento y a una frecuencia de resonancia la amplitud aumenta con el tiempo. En este caso se ha elegido un tiempo de respuesta de 10 segundos y los valores máximos se producirán cerca del final de este período como se puede ver en las figuras 2.6 y 2.7 . 


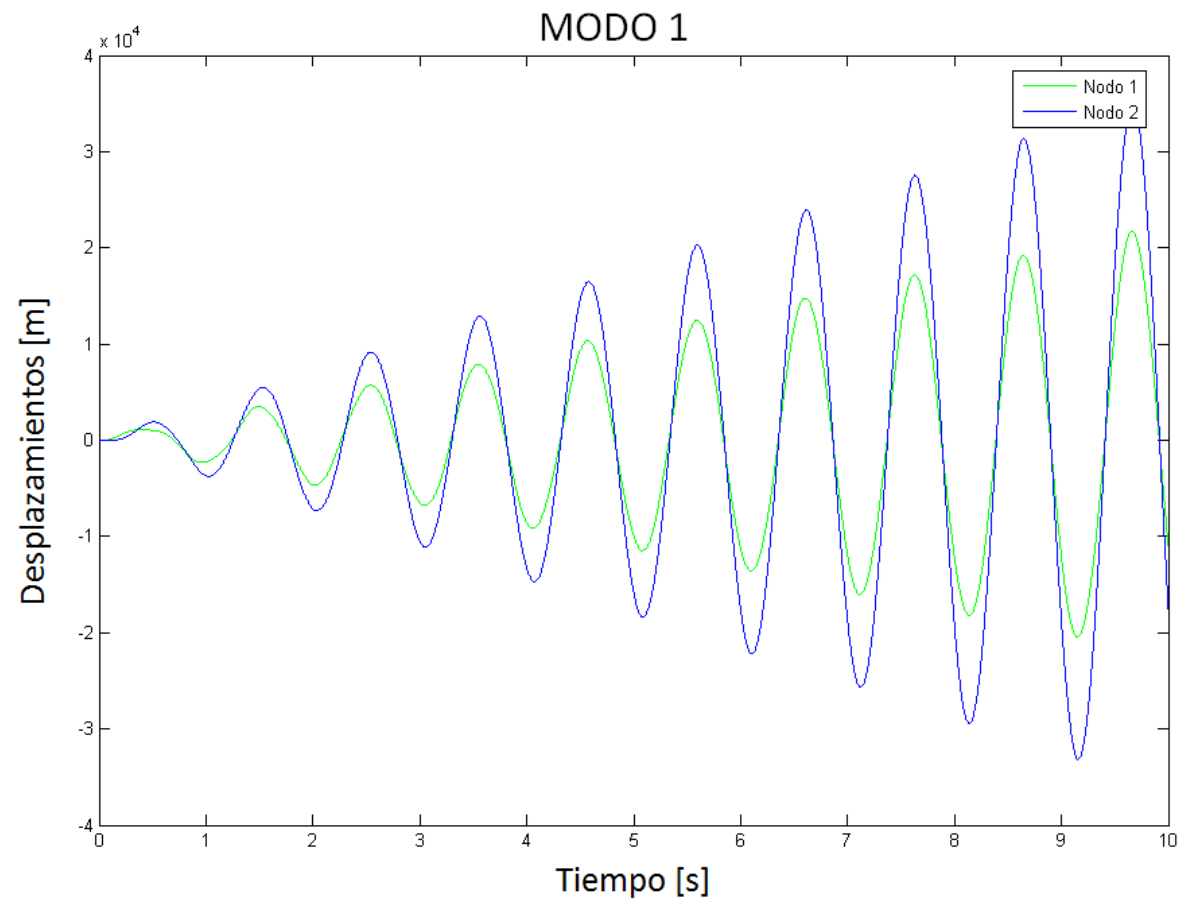

Figura 2.6: Desplazamientos en función del tiempo para el modo 1

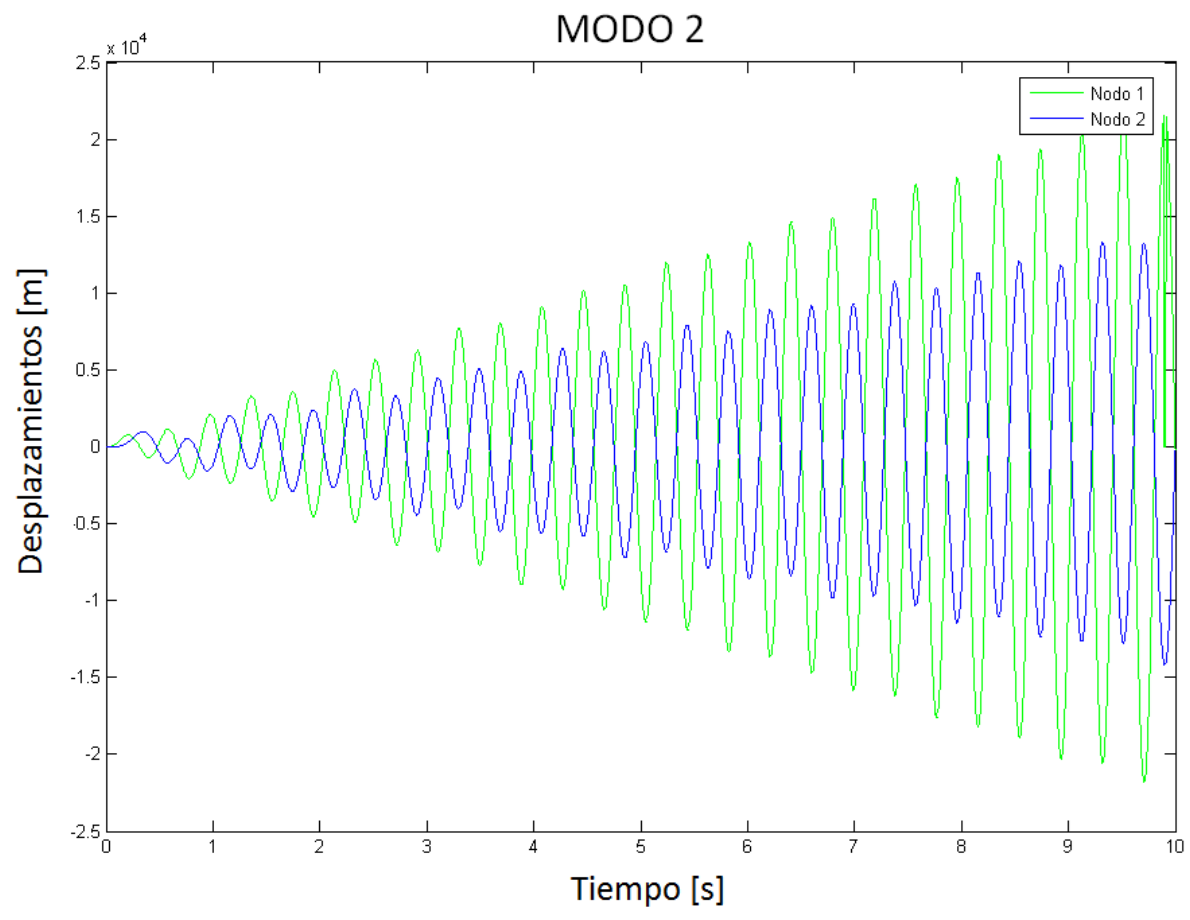

Figura 2.7: Desplazamientos en función del tiempo para el modo 2 


\subsection{Método de Craig-Bampton}

En la sección 1.2.1.1 se ha introducido la simplificación o reducción de este método en el modelo de elementos finitos. La demanda de recursos de cálculo normalmente está por encima de las capacidades existentes de los ordenadores actuales y esto reduce la capacidad para resolver grandes modelos que requieran largos tiempos de cálculo. Existen distintos métodos de simplificación como son el método de Guyan o la descomposición modal pero en este apartado se van a explicar los principios básicos del método de Craig-Bampton, ver Craig y Bampton (1968). El método se utiliza para reducir el tamaño de modelos de elementos finitos. Combina el movimiento de unos puntos en las condiciones de contorno con los modos propios de la estructura. Por lo tanto, la metodología para reducir una estructura que tiene una serie de puntos interfaz es la siguiente:

Se parte de la ecuación del movimiento, si ignoramos el amortiguamiento la ecuación es

$$
M y^{\prime \prime}+K y=F
$$

Si se pone en forma matricial

$$
[M]\left\{y^{\prime \prime}\right\}+[K]\{y\}=\{F\}
$$

La transformada de Craig-Bampton se define como:

$$
\left\{y_{A}\right\}=\left\{\frac{y_{b}}{y_{L}}\right\}=\left[\begin{array}{cc}
I & 0 \\
\phi_{R} & \phi_{L}
\end{array}\right]\left\{\begin{array}{c}
y_{b} \\
q
\end{array}\right\}
$$

donde $y_{b}$ son los grados de libertad de los puntos interfaz, $y_{L}$ son los grados de libertad de los puntos interiores, $\phi_{R}$ es el vector del sólido rígido, $\phi_{L}$ es la base de los modos y q son los grados de libertad de los modos a considerar. A partir de ahora se considera $\phi_{C B}=\left[\begin{array}{cc}I & 0 \\ \phi_{R} & \phi_{L}\end{array}\right]$. Si combinamos las ecuaciones anteriores y las premultiplicamos por $\left[\phi_{C B}\right]^{T}$ queda:

$$
\phi_{C B}^{T}\left[M_{A A}\right] \phi_{C B}\left\{\begin{array}{l}
y_{b}^{\prime \prime} \\
q^{\prime \prime}
\end{array}\right\}+\phi_{C B}^{T}\left[K_{A A}\right] \phi_{C B}\left\{\begin{array}{c}
y_{b} \\
q
\end{array}\right\}=\phi_{C B}^{T}\left\{\begin{array}{l}
F_{b} \\
F_{L}
\end{array}\right\}
$$

Si se definen las matrices $\left[M_{C B}\right]=\phi_{C B}^{T}\left[M_{A A}\right] \phi_{C B}=\left[\begin{array}{cc}M_{b b} & M_{b q} \\ M_{q b} & M_{q q}\end{array}\right]$ y $\left[K_{C B}\right]=\phi_{C B}^{T}\left[K_{A A}\right] \phi_{C B}=$ $\left[\begin{array}{cc}K_{b b} & 0 \\ 0 & K_{q q}\end{array}\right]$, entonces, la ecuación queda:

$$
\left[\begin{array}{cc}
M_{b b} & M_{b q} \\
M_{q b} & M_{q q}
\end{array}\right]\left\{\begin{array}{l}
y_{b}^{\prime \prime} \\
q^{\prime \prime}
\end{array}\right\}+\left[\begin{array}{cc}
K_{b b} & 0 \\
0 & K_{q q}
\end{array}\right]\left\{\begin{array}{c}
y_{b} \\
q
\end{array}\right\}=\left\{\begin{array}{c}
F_{b} \\
0
\end{array}\right\}
$$

donde las fuerzas sólo se pueden aplicar en la interfaz $\left(F_{L}=0\right)$. La matriz $\left[M_{b b}\right]$ es la matriz de masas trasladada a los puntos interfaz y la matriz $\left[K_{b b}\right]$ es la matriz de rigidez de los puntos de 
interfaz. Si el punto fuera uno sólo la matriz $K_{b b}=0$ y en el caso de que los modos estuvieran normalizados $\left[M_{q q}\right]$ sería la matriz identidad y $\left[K_{q q}\right]$ una matriz diagonal cuya diagonal tendría los valores $\lambda_{i}=k_{i} / m_{i}=\omega_{i}$.

En el caso de que se considerara que hubiera amortiguamiento y cumpliéndose las anteriores hipótesis la ecuación quedaría:

$$
\left[\begin{array}{cc}
M_{b b} & M_{b q} \\
M_{q b} & I
\end{array}\right]\left\{\begin{array}{l}
y_{b}^{\prime \prime} \\
q^{\prime \prime}
\end{array}\right\}+\left[\begin{array}{cc}
0 & 0 \\
0 & 2 \xi \omega
\end{array}\right]\left\{\begin{array}{l}
y_{b}^{\prime} \\
q^{\prime}
\end{array}\right\}+\left[\begin{array}{cc}
K_{b b} & 0 \\
0 & \omega^{2}
\end{array}\right]\left\{\begin{array}{c}
y_{b} \\
q
\end{array}\right\}=\left\{\begin{array}{c}
F_{b} \\
0
\end{array}\right\}
$$

donde $2 \xi \omega$ es el amortiguamiento modal. 


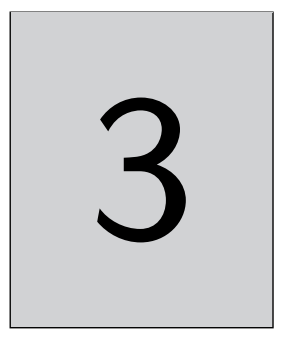

\section{FEnÓmenos DinÁmicos}

\section{Índice}

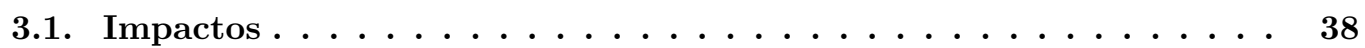

3.1.1. Ejemplo: Choque entre parejas de masas unidas por muelles . . . . . . . 39

3.1.2. Ejemplo: Impacto analizado con herramientas FEM avanzadas . . . . . 40

3.1.2.1. Introducción al ejemplo analizado . . . . . . . . . . . . . . . 40

3.1.2.2. Modelo FEM y análisis . . . . . . . . . . . . . . . . . 41

3.1.2.3. Conclusiones y recomendaciones . . . . . . . . . . . . 43

3.2. Frangibilidad ...................... 44

3.2.1. Ejemplo: Estructura frangible . . . . . . . . . . . . . . 44

3.2.1.1. Herramienta de análisis . . . . . . . . . . . . . . . 45

3.2.1.2. Correlación del modelo con el ensayo de carga lateral . . . . . 46

3.2.1.3. Validación de las propiedades mecánicas y el criterio de fallo . 47

3.2.1.4. Resultados de frangibilidad de los ensayos . . . . . . . . . . . . 48

3.3. Incidentes de vuelo; pérdida de pala de un avión de transporte . • 50

3.3.1. Análisis del fenómeno de la pérdida de pala . . . . . . . . . . . . . 51

3.3.1.1. Fenómeno físico de la pérdida de pala . . . . . . . . . . . . 52

3.3.2. Ejemplo: Viga en voladizo con carga armónica seguidora . . . . . . . . . 54

En ingeniería el análisis de fallos consiste en determinar la causa del fallo con el fin de aumentar la fiabilidad o mejorar y optimizar el rendimiento y el diseño de una estructura dada. En un análisis dinámico hay que tener en cuenta parámetros nuevos, principalmente las fuerzas de inercia. Si se aplica una carga con suficiente lentitud, las fuerzas de inercia se puede ignorar, y el análisis se puede simplificar. Cualquier estructura puede ser sometida a una carga dinámica; sólo necesita una carga que cambie rápidamente con el tiempo en comparación con la frecuencia natural del sistema. Por lo tanto, un análisis dinámico de fallos consiste en determinar la causa de un fallo cuando se somete a una carga dinámica. Ejemplos de cargas dinámicas son un impacto, vibraciones, etc. En el caso de los aviones, las cargas normalmente son dinámicas y se dividen en dos categorías principales: las cargas límite y las cargas últimas. Las cargas límite son cargas que se encontrará la estructura a lo largo de su vida útil, cargas de la envolvente de vuelo común (con maniobras, ráfagas,...). Cargas últimas son las cargas que la estructura tiene que soportar un período corto de tiempo, por ejemplo, las cargas de choque, y ciertos 
daños son permitidos en la estructura aunque nunca un fallo catastrófico. Por lo tanto, las cargas de maniobras se determinan en base a su vida útil y las actuaciones de la aeronave, y las ráfagas se determinan estadísticamente mediante las directrices y requisitos de las autoridades de certificación. Por el contrario, las cargas de choque están delimitadas por los objetivos de diseño; si la estructura no está tripulada estos niveles pueden ser más altos ya que no estarán limitados por la capacidad humana para resistir cierto nivel de aceleraciones. En el caso de un avión turbohélice, la pérdida de pala no puede desembocar en un grave accidente y el avión debe ser capaz de completar con éxito el vuelo. Otras cargas que han de tenerse en cuenta son las de tierra. Pueden provenir de fuentes muy diversas, desde posición en reversa del motor o maniobras durante el rodaje que pueden producir impactos de restos de la pista de rodadura. Estos últimos tipos de incidentes están conectados directamente con el concepto de tolerancia al daño. La tolerancia al daño es una propiedad de una estructura relacionada con su capacidad para soportar los daños de forma segura hasta su posible reparación. Hay que garantizar el funcionamiento seguro de la estructura ante casos de carga a priori inofensivos. El daño puede surgir incluso en impacto de baja velocidad como el caso de herramienta que se le cae a un operario cuando está realizando el mantenimiento de la estructura. Por otro lado está el concepto frangibilidad donde la estructura debe dañarse y romperse fácilmente. En un aeropuerto, las estructuras cercanas a la pista de aterrizaje deben ser frangible, éste es el caso de balizas o marcadores de aeropuerto. En particular, una estructura frangible debe romperse, doblarse o deformarse cuando se le somete a fuerzas debidas a un choque. En este capítulo se discute el análisis de fallos para diferentes casos muy representativos:

- Un impacto de baja velocidad sobre una estructura de material compuesto que puede ser debida a la caída de una herramienta o por impacto de los restos de una pista de despegue.

- Un impacto en un aeropuerto con una estructura frangible debido a un incidente en tierra.

- Una pérdida de pala de un avión turbohélice debido a un daño, una grieta en el buje de la hélice.

Además de estos casos se introducen varios ejemplos (choque de masas y viga bajo carga armónica) con los que se introducen las herramientas que luego se utilizan en los eventos descritos. Cada caso de estudio utiliza una estrategia diferente para analizar el fallo debido a las distintas naturalezas de los sucesos. Sin embargo, todos ellos incluyen una simulación de fallo altamente dinámico, que puede implicar también la simulación de la secuencia del fallo.

\subsection{Impactos}

Uno de los fenómenos dinámicos más concurrentes en las estructuras de hoy en día son los impactos por otros objetos. Estos fenómenos pueden producir consecuencias de diversas índoles 
y hay que estudiar en profundidad la vida útil de la estructura para analizar los impactos que puede sufrir con una cierta probabilidad.

Estos impactos pueden producir en las estructuras desde plastificaciones locales, pandeos locales e incluso globales y roturas del material. Los fenómenos pueden ser tan complejos que impliquen grandes deformaciones y rotaciones con contactos entre varios componentes. Estas deformaciones suelen iniciarse mediante ondas asociadas a grandes esfuerzos. Puede suceder que estos esfuerzos superen el valor admisible en las primeras ondas. A continuación les siguen los efectos inerciales de la respuesta transitoria. En este caso son muy importantes parámetros como los esfuerzos, deformaciones y la absorción de energía. En este punto se pueden distinguir distintos impactos dependiendo de la velocidad. Impactos de baja o media velocidad que son lo que van desde $5 \mathrm{~km} / \mathrm{h}$ hasta casi $200 \mathrm{~km} / \mathrm{h}$. Su duración debe ser corta, de entre 100 y $200 \mathrm{~ms}$.

\subsubsection{Ejemplo: Choque entre parejas de masas unidas por muelles}

Dos masas $\mathrm{m}$ viajan unidas por un muelle de constante $\mathrm{k}$. Llevan una velocidad igual $v_{0}$ antes de chocar contra otra pareja de masas $\mathrm{m}$ unidas por otro muelle de igual constante $\mathrm{k}$ como se puede ver en la figura 3.1. Los datos del problema son constante del muelle $k=10^{10}$

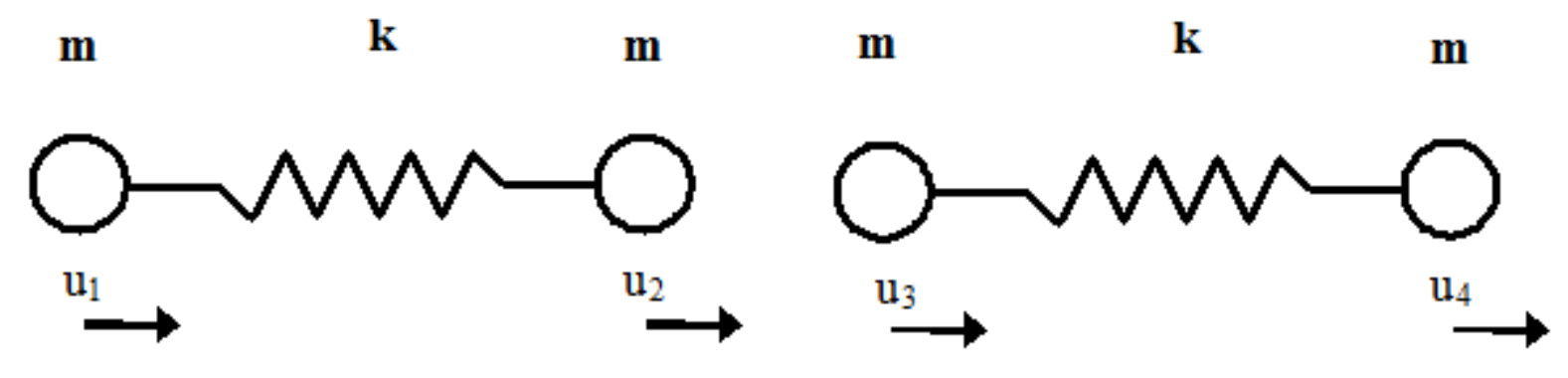

Figura 3.1: Choque entre masas unidas por muelles

$[\mathrm{N} / \mathrm{m}]$, masas $m=1[\mathrm{Kg}]$, longitud natural del muelle $L_{0}=0,01[\mathrm{~m}]$, distancia inicial entre las masas más cercanas $D=0,005[\mathrm{~m}]$, velocidad inicial de las masas $v_{0}=100[\mathrm{~m} / \mathrm{s}]$ y parámetro de contacto $\epsilon=0,0001[\mathrm{~m}]$. Antes del impacto las ecuaciones del movimiento son:

$$
\left(\begin{array}{cccc}
m & 0 & 0 & 0 \\
0 & m & 0 & 0 \\
0 & 0 & m & 0 \\
0 & 0 & 0 & m
\end{array}\right)\left\{\begin{array}{l}
\ddot{u}_{1} \\
\ddot{u}_{2} \\
\ddot{u}_{3} \\
\ddot{u}_{4}
\end{array}\right\}+\left(\begin{array}{cccc}
k & -k & 0 & 0 \\
-k & k & 0 & 0 \\
0 & 0 & k & -k \\
0 & 0 & -k & k
\end{array}\right)\left\{\begin{array}{l}
u_{1} \\
u_{2} \\
u_{3} \\
u_{4}
\end{array}\right\}=\{0\}
$$

Estas ecuaciones se integran con un esquema implícito de $\beta$-Newmark cuyas ecuaciones están en 5.2. Para controlar el impacto se define un parámetro $\epsilon$ como condición de contacto $u_{2}-u_{3}<$ 
$\epsilon$. Aplicando la condición de conservación de la cantidad de movimiento $\dot{u}_{3 d e s p}=\dot{u}_{2 \text { desp }}=$ $m /(2 m) \dot{u}_{2 a n t}$. Las ecuaciones pasan a ser:

$$
\left(\begin{array}{ccc}
m & 0 & 0 \\
0 & 2 m & 0 \\
0 & 0 & m
\end{array}\right)\{\ddot{u}\}+\left(\begin{array}{ccc}
k & -k & 0 \\
-k & 2 k & k \\
0 & -k & k
\end{array}\right)\{u\}=\{0\}
$$

En la figura 3.2 se muestran los resultados de las posiciones de las masas en función del tiempo para $\Delta t$ de $10^{-5}, 10^{-6}$ y $10^{-7} \mathrm{~s}$. La diferencia entre los resultados de $10^{-6}$ y $10^{-7}$ es despreciable siendo dichos resultados adecuados no así los de $10^{-5}$ en los que se refleja la influencia de un $\Delta t$ insuficiente para representar la solución.
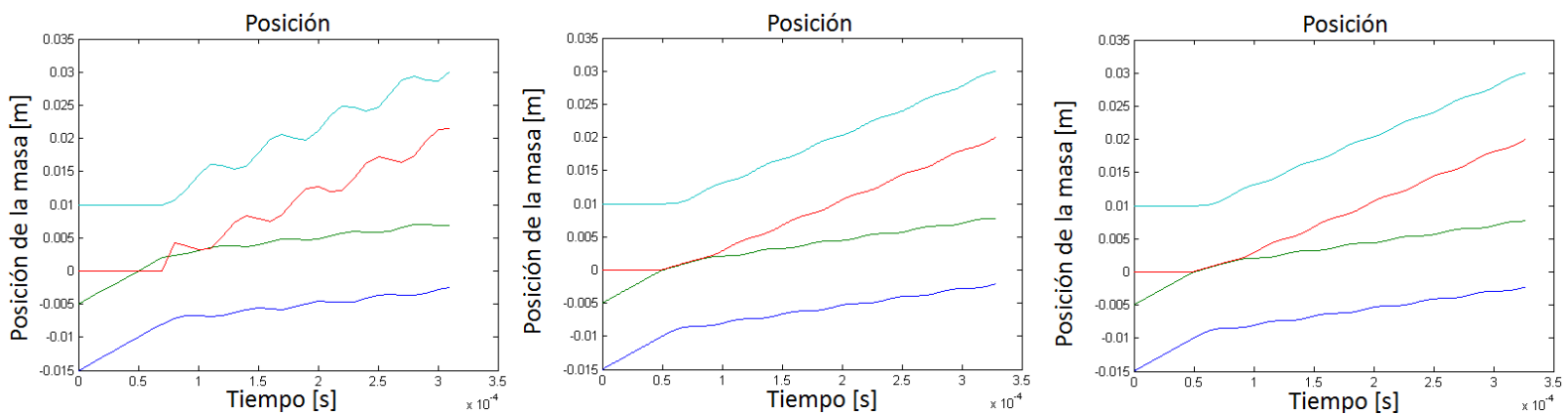

Figura 3.2: Posición en función del tiempo para $\Delta t 10^{-5}, 10^{-6}$ y $10^{-7} \mathrm{~s}$

\subsubsection{Ejemplo: Impacto analizado con herramientas FEM avanzadas}

A continuación se muestra el estudio realizado a un problema de impacto en una estructura de material compuesto.

\subsubsection{Introducción al ejemplo analizado}

Las estructuras de material compuesto están cada vez más extendidas tanto en el campo de las aeronaves civiles como militares, y recientemente en los drones, o vehículos aéreos no tripulados (UAV), o vehículos pilotados por control remoto (RPA). Sin embargo, estas estructuras son sensibles a impactos a baja velocidad que pueden producir múltiples delaminaciones en la dirección del espesor, así como agrietamiento de la matriz, rotura de fibras, delaminación translaminar, u otro tipo de fallos Richardson y Wisheart (1996). No existe todavía una metodología lo suficientemente madura, fiable y contrastada que sea capaz de predecir el inicio del daño y su propagación, sin embargo hay muchos estudios Kärger et al. (2009) y revisiones del estado de la técnica Elder et al. (2004). En muchos casos, el crecimiento del daño es tan rápido que no es predecible y para el proceso de certificación se utiliza el concepto de "no-growth", que no haya crecimiento del daño. Por este motivo estos análisis suelen ser muy costosos y lentos computacionalmente. 
Además, el daño por impacto es muy complejo y los resultados son variables e irregulares; múltiples delaminaciones, agrietamiento de la matriz, etc, Xu y Chen (2013); Shi et al. (2012), y huellas permanentes y otro tipo de distorsiones. Asimismo, los tipos de daño dependen de varios parámetros como el laminado, el material o las condiciones del impacto. Debido a la complejidad del daño, es necesario una simplificación para poder centrarse en las características más importantes. El objetivo principal es desarrollar y examinar métodos que evalúen el daño por impacto y predecir la resistencia residual. En este caso de estudio, se muestra una comparación de la simulación y los resultados de ensayo. La metodología de simulación mediante el método de elementos finitos (FEM) es capaz de predecir y caracterizar el daño producido en paneles de material compuesto en impactos de baja y media energía. El trabajo está enfocado en paneles con daños difícilmente visibles (BVID).

\subsubsection{Modelo FEM y análisis}

Los paneles son de dos materiales, ambos de fibras de carbono y resina epoxi, IM7/9772 y IM7/8552. Se tienen en cuenta dos configuraciones de laminado u cada una de ellas con diferente tamaño y energía de impacto $(11 \mathrm{~J} \mathrm{y} 20 \mathrm{~J}$ ). El espesor de los paneles es de $3 \mathrm{~mm}$ y la secuencia de apilamiento $\left[(-45,+45,90,0)_{3}\right]_{S}$. El caso de estudio está también explicado en Falzon (2006). Los paneles FEM se modelan utilizando el pre/post MSC.Patran y se utiliza el método implícito MSC.Marc para resolver el análisis, MSC (2005). MSC.Marc que es un solver de análisis estructural multidisciplinar que utiliza métodos de resolución implícitos para realizar análisis estáticos y dinámicos y está muy enfocado a la simulación de problemas no lineales.

El análisis FEM tiene las siguientes características: no-lineal transitorio, con daño progresivo bajo criterios de fallo basados en esfuerzos. El criterio de fallo tiene en cuenta los siguientes modos de fallo: rotura de fibra, micro pandeo de fibras, rotura de la matriz (por tensión, compresión, y cortadura en el plano), fallo por esfuerzos fuera del plano, y fallo por esfuerzos interlaminares. Para todos estos modos de fallo se utiliza el criterio de máximo esfuerzo. Los elementos que representan el panel son elementos sólidos. El panel está empotrado en ejes UX, UY y UZ a lo largo de anillos a $140 \mathrm{~mm}$ y $220 \mathrm{~mm}$. El modelo FEM se puede ver en la figura 3.3 .

Los resultados FEM se han estudiado para cada índice de fallo de cada modo de fallo y en cada capa de laminado. Los resultados experimentales muestran la zona dañada aunque no indica cuál es el tipo de fallo que ha causado ese daño (delaminaciones, agrietamiento de la matriz, rotura de fibras) por lo tanto las comparaciones entre los ensayos y los análisis FEM son en cada capa pero a nivel de daño global sin poder comparar los producidos por distintos tipos de fallo. En la figura 3.4 se compara el daño. En todas las imágenes del FEM, que han sido reescaladas para permitir la comparación, se observa una clara correspondencia con las imágenes del daño en los ensayos. El color rojo de las imágenes FEM significa que el fallo ha ocurrido 


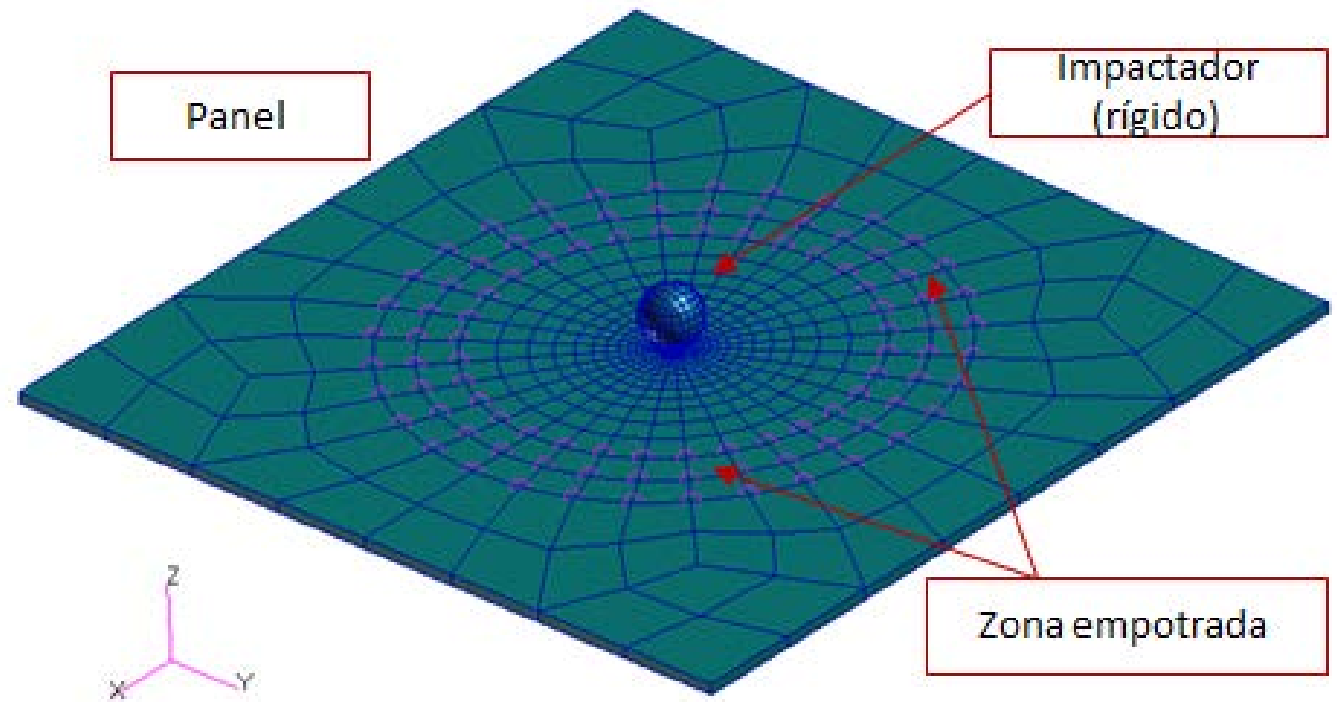

Figura 3.3: Modelo de elementos finitos

en al menos uno de los modos de fallo considerados, y que ha sucedido en al menos una de las capas.
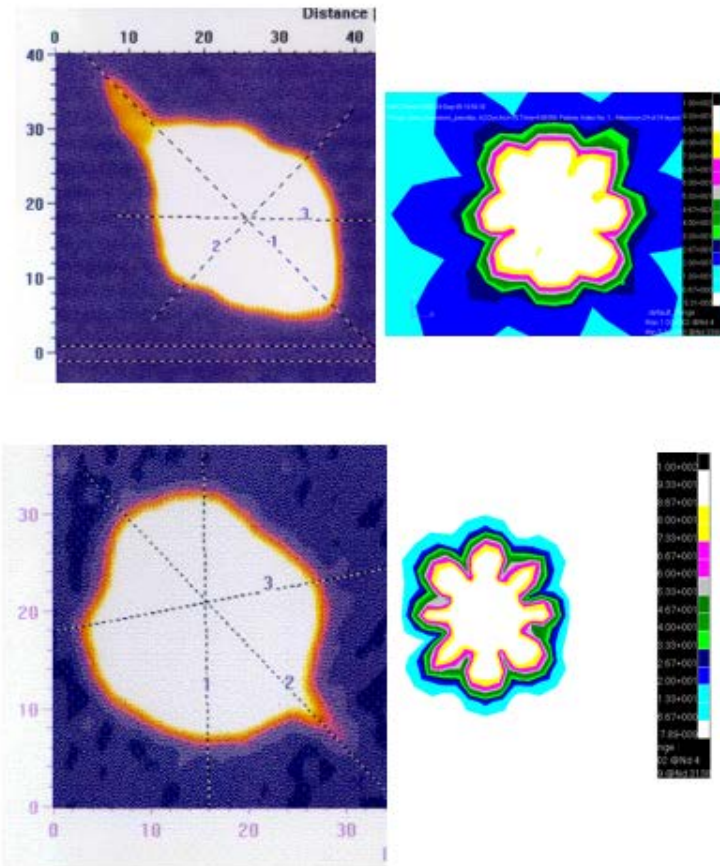
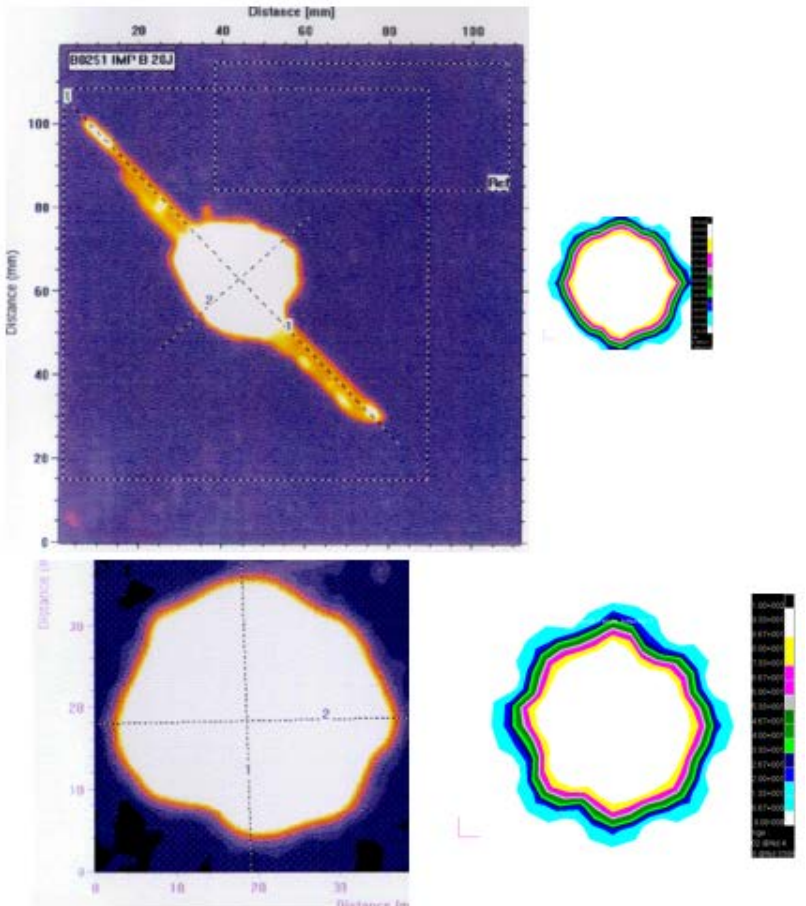

Figura 3.4: Comparación entre el modelo FEM y los ensayos 
Igualmente, se ha realizado una correlación entre las deflexiones en función del tiempo del impactador del ensayo y del análisis. En la figura 3.5 se muestra este resultado para los diferentes paneles.
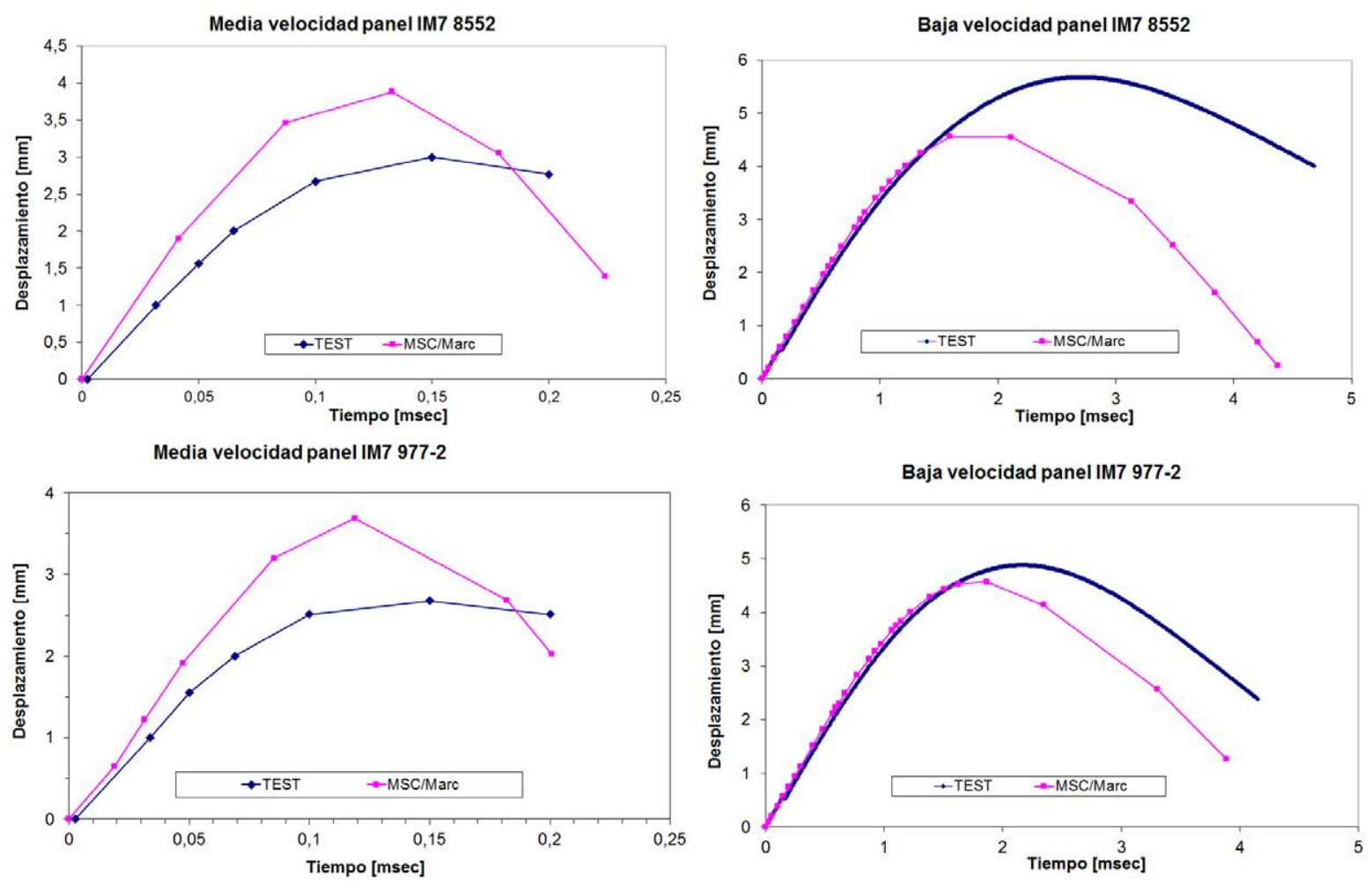

Figura 3.5: La deflexión en función del tiempo para diferentes paneles y casos de carga

Las siguientes conclusiones se obtienen de la correlación FEM-ensayo: la predicción del área dañada se puede considerar satisfactoria para el material IM7/8552 y sólo aceptable para el caso de IM7/977-2. Parece que el admisible escogido para $\sigma_{z z}$ es alto y debería ser reevaluado o confirmado con otras fuentes. Si el admisible $\sigma_{z z}$ fuera más bajo, el área dañada crecería y correlaría mejor con los resultados de los ensayos. Las gráficas de la deflexión del impactador en función del tiempo muestran una muy buena correlación para impactos de baja velocidad y paneles de IM7/977-2 y resultados sólo aceptables en los demás. La conclusión general es que la metodología FEM utilizada parece adecuada y las correlaciones entre las simulaciones y los ensayos son como poco aceptables, y muy satisfactorias en algunos casos.

\subsubsection{Conclusiones y recomendaciones}

La predicción de la respuesta al impacto y daño en paneles planos se ha llevado a cabo con un modelo de formulación cerrada y mediante elementos finitos. El modelo predice el crecimiento de la delaminación utilizando criterios de mecánica de fractura (máximo esfuerzo). El modelo FEM se ha mostrado que puede ser una herramienta de diseño rápido utilizando mallas de ele- 
mentos relativamente groseras y un criterio de fallo basado en esfuerzos. Antes de la penetración y rotura la simulación predice la respuesta y la extensión de la zona delaminada en impactos de caída con cierta precisión. La mayor limitación que se ha encontrado es la incapacidad de predecir comparando la forma de cada delaminación individual y el área de otros tipos de fallo. Otro inconveniente se ha encontrado en los datos de admisibles de material. Para finalizar, se recomienda o bien seguir utilizando modelos que predigan el crecimiento de las delaminaciones en criterios basados en la mecánica de fractura, o utilizar otras aproximaciones utilizando la absorción de energía durante el crecimiento de la delaminación.

\subsection{Frangibilidad}

Otra aplicación en la que los fallos en análisis dinámicos están muy presentes es la frangibilidad en estructuras de aeropuertos. No hay muchos estudios en este campo de aplicación debido a la complejidad de las simulaciones Wiggenraad et al. (2000); Wiggenraad y Zimcik (2001). La frangibilidad se define como la propiedad que permite a un objeto romper, deformar o plastificar una estructura en un impacto bajo ciertas condiciones mientras absorbe una mínima energía, para que así sea un mínimo riesgo para una aeronave en caso de impacto. La mayoría de los suministradores realiza ensayos para cumplir los requisitos. En el documento ICAO 'Aerodrome Design Manual' parte VI acerca de frangibilidad ICAO (2006) se describen los requerimientos mínimos para que una estructura se considere frangible.

- Cerca de las pistas de despegue en los aeropuertos los objetos deben ser frangibles. Tienen que poder soportar cargas aerodinámicas, de chorro de motor, tanto en estático como en operación con el correspondiente factor de seguridad, sin embargo, debe romperse o doblarse fácilmente cuando sufra una colisión con una aeronave de $3000 \mathrm{~kg}$ en el aire y volando a $140 \mathrm{~km} / \mathrm{h}$ o moviéndose en tierra a $50 \mathrm{~km} / \mathrm{h}$. Por lo tanto, se realizan ensayos a escala real o simulaciones en estas estructuras frangibles para determinar su comportamiento.

- La frangibilidad se consigue mediante el uso de materiales ligeros o introduciendo algún mecanismo de separación que permita al objeto romperse o doblarse en el impacto.

- En el caso de vigas o mástiles, la estructura se considera frangible cuando la energía de impacto se limita a $55 \mathrm{~kJ}$ y el pico de fuerza no supera $45 \mathrm{kN}$.

\subsubsection{Ejemplo: Estructura frangible}

A continuación se muestra el estudio realizado para atacar un problema con estas características. El mástil considerado es un tubo de $12 \mathrm{~m}$ de longitud que se inserta en un agujero cónico en el suelo. El mástil se utiliza como un soporte de luces en la zona de la pista de despegue y aterrizaje. Consiste en una estructura compuesta de fibra de vidrio y resina poliéster. En la zona 
inferior del poste son 6 capas de $1.6 \mathrm{~mm}$, que van gradualmente desapareciendo para quedar solamente 3 en la parte superior. La base está hecha de fibra de vidrio $\left(0^{\circ} / 90^{\circ}\right)$, fibras cortas con resina de poliéster. En la parte más estrecha de la base hay 9 capas y 18 en la siguiente zona hasta la zona plana que también tiene 18. El análisis consiste en un impacto a $50 \mathrm{~km} / \mathrm{h}$ (avión en tierra rodando) y $140 \mathrm{~km} / \mathrm{h}$ (avión en vuelo aterrizando/despegando). En las simulaciones el avión se modeliza como un cilindro rígido de $3000 \mathrm{~kg}$.

\subsubsection{Herramienta de análisis}

Para llevar a cabo estas simulaciones de impactos se utiliza el solver MSC.Dytran que tiene un esquema explícito, MSC (2005). MSC.Dytran es un software basado en un método explícito de diferencias centradas que simula la respuesta dinámica de sistemas que pueden ser sólidos y/o fluidos. Combina la metodología de elementos finitos de sólidos con la de mecánica de fluidos para facilitar la modelización del problema. El software incluye numerosos tipos de material y no linealidades geométricas. El pre-solver utilizado es MSC.Patran. Como se ha mencionado anteriormente los métodos explícitos se utilizan para simular fenómenos de corta duración, como impactos, simulaciones de choques cuando la onda de propagación y la componente dinámica son importantes. El caballo de batalla de estos solvers es el gran número de pasos de tiempo necesarios por el paso de tiempo crítico y, por lo tanto, el tiempo CPU que consume cada caso. En este caso el modelo consiste en 2395 elementos placa:

- Columna: Es un tubo con una sección que se va estrechando, modelada por elementos placa de material compuesto unidireccional y resina poliéster. El acceso inferior del cableado no se ha considerado ya que influye poco y si se tuviera en cuenta haría a la columna aún más frágil. Cada capa tiene $1.6 \mathrm{~mm}$. Con estas propiedades se lamina la columna con distintas configuraciones. Las propiedades se muestran en la tabla 3.1.

- Impactador: Es un cilindro rígido de $0.4 \mathrm{~m}$ de diámetro. Su centro se sitúa a $3 \mathrm{~m}$ sobre la base de la columna y está formado por un material infinitamente rígido con una masa total de $3000 \mathrm{~kg}$.

- Base: Malla de elemento placa con dos áreas diferenciadas. La zona con estrechamiento en contacto con el poste tiene 9 capas de material compuesto y el collar y la base tienen 18 . Tela (0/90) de fibra de vidrio y fibra corta con resina poliéster en cada capa. Se modela con las propiedades de los resultados obtenidos de ensayos.

- Suelo: Además de los elementos mencionados la base se sitúa a nivel del suelo. El suelo se modela ya que cuando el poste se sale de la base cónica durante el impacto no puede entrar dentro y atravesar el suelo. Está formado por una malla 2D cuyos nodos están apoyados. 


\begin{tabular}{|c|c|c|}
\hline & COLUMNA & BASE \\
\hline Propiedad & Unidades S. I. & Unidades S. I. \\
\hline Densidad & 1600 & 1261 \\
\hline Módulo de Young $E_{11}$ & $1.7 \mathrm{E}+10$ & $1.1 \mathrm{E}+10$ \\
\hline Módulo de Young $E_{22}$ & $6 \mathrm{E}+10$ & $1.1 \mathrm{E}+10$ \\
\hline Coeficiente de Poisson 12 & 0.3 & 0.198 \\
\hline Módulo de cortadura $G_{12}$ & $6.5 \mathrm{E}+9$ & $4.59 \mathrm{E}+9$ \\
\hline Módulo de cortadura $G_{1 z}$ & $6.5 \mathrm{E}+9$ & $4.59 \mathrm{E}+9$ \\
\hline Módulo de cortadura $G_{2 z}$ & $6.5 \mathrm{E}+9$ & $4.59 \mathrm{E}+9$ \\
\hline Esfuerzo crítico & $6 \mathrm{E}+7$ & \\
\hline Fuerza de tensión longitudinal máxima & $2 \mathrm{E}+8$ & $2.14 \mathrm{E}+8$ \\
\hline Fuerza de compresión longitudinal máxima & $1.8 \mathrm{E}+8$ & \\
\hline Fuerza de tensión lateral máxima & $1.04 \mathrm{E}+8$ & $2.14 \mathrm{E}+8$ \\
\hline Fuerza de compresión lateral máxima & $1.4 \mathrm{E}+8$ & \\
\hline
\end{tabular}

Tabla 3.1: Propiedades de los materiales en mástil y base

Las condiciones de contorno consisten en 4 puntos de la parte cuadra de la base que están empotrados. Las velocidades iniciales son 50 y $140 \mathrm{~km} / \mathrm{h}$ y se aplican al impactador. Finalmente, se aplican las condiciones de contacto del problema (ver figura 3.6):

- Impactador-columna

- Columna-base. En este caso se aplica una fricción entre ellos, 0.6 de fricción estática y 0.3 de dinámica.

- Columna con ella misma. Cuando la columna se dobla por el impacto y colapsa, los elementos pueden llegar a contactar con otros de la misma columna y no se permite esa penetración.

- Columna-suelo. Cuando la columna se sale de la base no puede atravesar el suelo.

\subsubsection{Correlación del modelo con el ensayo de carga lateral}

Se realizó un ensayo con carga lateral que obtuvo los siguientes resultados:

- Carga nominal: $870 \mathrm{~N}$ con una deflexión de $1225 \mathrm{~mm}$.

- Carga última: $2580 \mathrm{~N}$.

Se llevan a cabo una serie de simulaciones cuasi-estáticas de la columna sometida a la carga lateral (aplicada en la punta) incrementándose de 0 a 2500 N. Esto se utiliza para validar las 


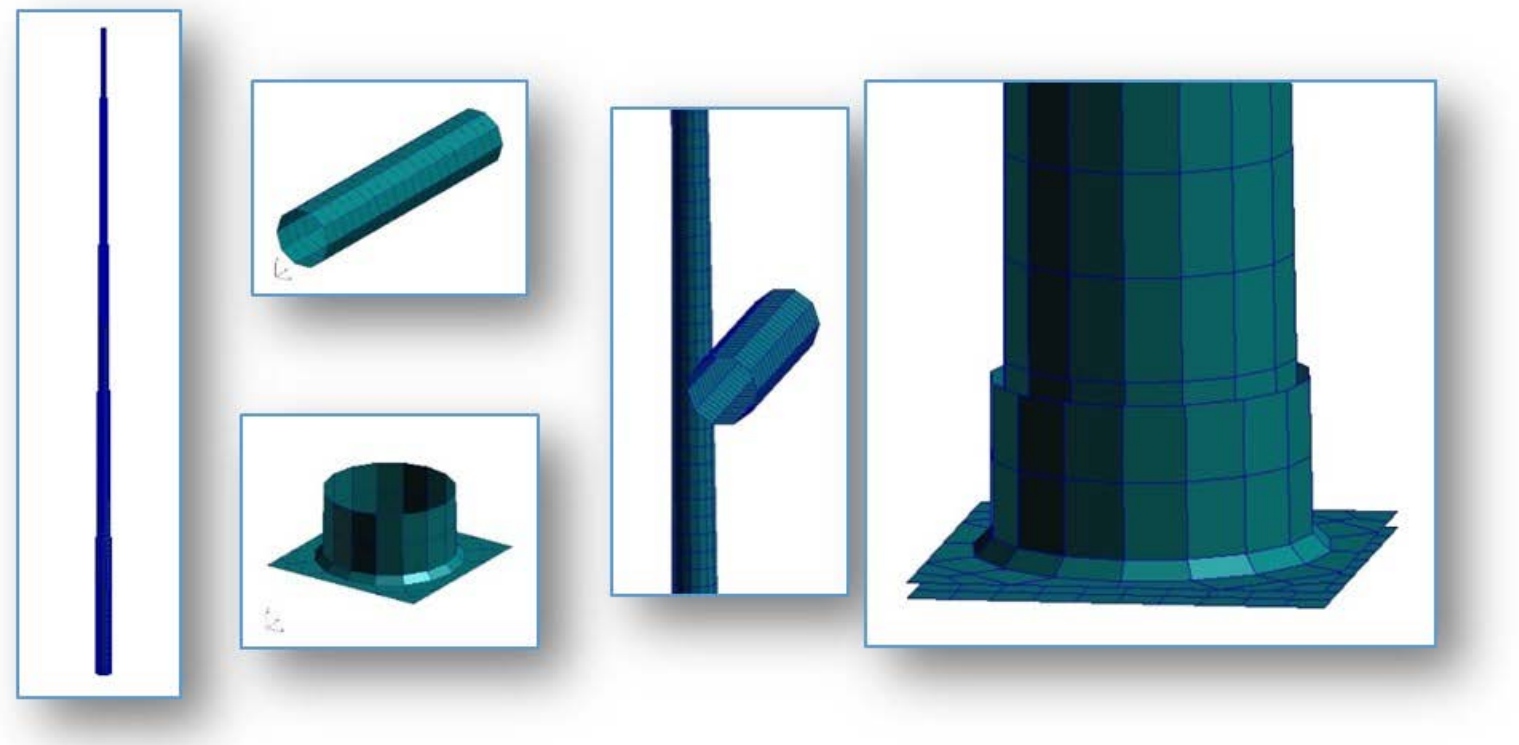

Figura 3.6: Columna, impactador y base. Contacto impactador-columna y columna-base

propiedades de material, el coeficiente de fricción entre la base y la columna y el criterio de fallo seleccionado. Los análisis se realizaron en MSC.Dytran por lo que tenía en cuenta las fuerzas de inercia. Se introdujo amortiguamiento en el sistema para reducir estas fuerzas y obtener unos resultados más fiables y con un tiempo computacional aceptable.

\subsubsection{Validación de las propiedades mecánicas y el criterio de fallo}

Se ha realizado una serie de ensayos con carga lateral. Esta carga es no es lo suficientemente grande como para causar algún daño en el material. La deflexión se ha medido y cotejado con la simulada en el modelo donde tampoco hay ningún daño. De estas comparaciones se obtiene el amortiguamiento a aplicar al resto de las simulaciones. Los análisis que se han llevado a cabo son:

- Simulación de $1 \mathrm{~s}$ con un incremento de carga hasta los $100 \mathrm{~N}$ a los $0.5 \mathrm{~s}$, y constante durante el resto. Se obtuvo una deflexión en punta entre 0.125 y 0.175 m.

- Simulación de $0.5 \mathrm{~s}$ con una carga constante de 100 N. Se obtuvo un valor de $0.125 \mathrm{~m}$.

El valor de la deflexión en punta durante el ensayo nominal $870 \mathrm{~N}(1.225 \mathrm{~m})$ se extrapola al ensayo suponiendo linealidad y se obtiene $0.140 \mathrm{~m}$ para los $100 \mathrm{~N}$. Los valores obtenidos son comparables por lo que los valores de las propiedades introducidas son adecuados. Se han introducido valores prácticamente despreciables de amortiguamiento (0.00002) para reducir el tiempo de cálculo. La batería de análisis incluía diferentes introducciones de carga (constante y rampa) e incremento hasta los $2500 \mathrm{~N}$ (ver tabla 3.2). Las deflexiones a carga nominal son evaluadas y 
contrastadas con el ensayo. Además, se obtienen los valores de carga última de la columna o de la separación de la columna de la base. Igualmente, la carga se aplica gradualmente y se introduce el amortiguamiento. Las simulaciones se comportan linealmente en la carga nominal. En el

\begin{tabular}{|c|c|c|c|c|c|}
\hline $\mathbf{t}[\mathbf{s}]$ & Carga (t) $[\mathbf{N}]$ & Amortiguamiento & $\mathbf{1 0 0} \mathbf{~ N}$ & $\mathbf{8 7 0} \mathbf{~ N}$ & $\mathbf{2 5 0 0} \mathbf{~ N}$ \\
\hline 2 & $0(0 \mathrm{~s}) / 2500(2 \mathrm{~s})$ & 0.00002 & & 0.91 & 7 \\
\hline 2 & $0(0 \mathrm{~s}) / 2500(2 \mathrm{~s})$ & 0.00001 & & 1.06 & 9 \\
\hline 2 & $0(0 \mathrm{~s}) / 2500(2 \mathrm{~s})$ & 0.000005 & & 1.11 & 9 \\
\hline 10 & $0(0 \mathrm{~s}) / 2500(10 \mathrm{~s})$ & 0.00002 & & 1.08 & 7.5 \\
\hline
\end{tabular}

Tabla 3.2: Los resultados más relevantes de la simulación

ensayo, la deflexión en la carga nominal $(1.225 \mathrm{~m})$ se comparó con las obtenidas en los ensayos $(0.91,1.06,1.11,1.08)$, por lo que la simulación es desde un $10 \%$ a un $35 \%$ más conservativa. En el caso de simular 2 segundos (introduciendo amortiguamiento a efectos computacionales) la rotura se produce a los $2500 \mathrm{~N}$. En la parte superior del poste hay elementos que empiezan a romperse y las capas se empiezan a separar. La columna empieza también a salirse de la base pero no se produce el fallo global. Por lo tanto, los resultados de la columna son desde el punto de vista de la frangibilidad conservativos, en tanto en cuanto la columna no se rompe o se sale de la base totalmente a los valores de carga última de los ensayos.

\subsubsection{Resultados de frangibilidad de los ensayos}

La normativa requiere 2 tipos de análisis de frangibilidad: el caso de avión rodando en pista a $50 \mathrm{~km} / \mathrm{h}$ (ver figura 3.7) y el caso de avión volando en pista a $140 \mathrm{~km} / \mathrm{h}$ (ver 3.8). Los resultados a $50 \mathrm{~km} / \mathrm{h}$ correlan y se espera un comportamiento en el que la columna se dobla y se sale de la base. El modo de fallo es la separación de las partes. A $140 \mathrm{~km} / \mathrm{h}$ los resultados correlan, en este caso el impactador atraviesa la columna. El modo de fallo es por fragilidad de la columna, fallo de elementos. La energía perdida por el impactador, que es absorbida por la columna, es $20.6 \mathrm{~kJ}$ a $50 \mathrm{~km} / \mathrm{h}$ y $20.3 \mathrm{~kJ}$ a $140 \mathrm{~km} / \mathrm{h}$. Los niveles de energía son mucho menores que los permitidos (55 kJ), (ver figura 3.9). Esto es debido al bajo módulo de elasticidad de las fibras de vidrio y el comportamiento frágil del material. Además, la energía de hourglass es menor al $10 \%$ del total a lo largo de toda la simulación. Por lo tanto, los resultados son adecuados desde el punto de vista de análisis explícito. La fuerza de impacto máxima en el caso de $50 \mathrm{~km} / \mathrm{h}$ es de $27 \mathrm{kN}$ y la columna se separará completamente de la base en $133 \mathrm{~ms}$ (ver figura 3.10). En el caso de $140 \mathrm{~km} / \mathrm{h}$, la columna se rompe antes de $30 \mathrm{~ms}$ y el máximo de fuerza alcanza aproximadamente $50 \mathrm{kN}$. La carga máxima que se obtiene en el impacto es similar a la máxima especificada $45 \mathrm{kN}$. Por lo tanto, se puede concluir que se puede considerar al mástil frangible. 


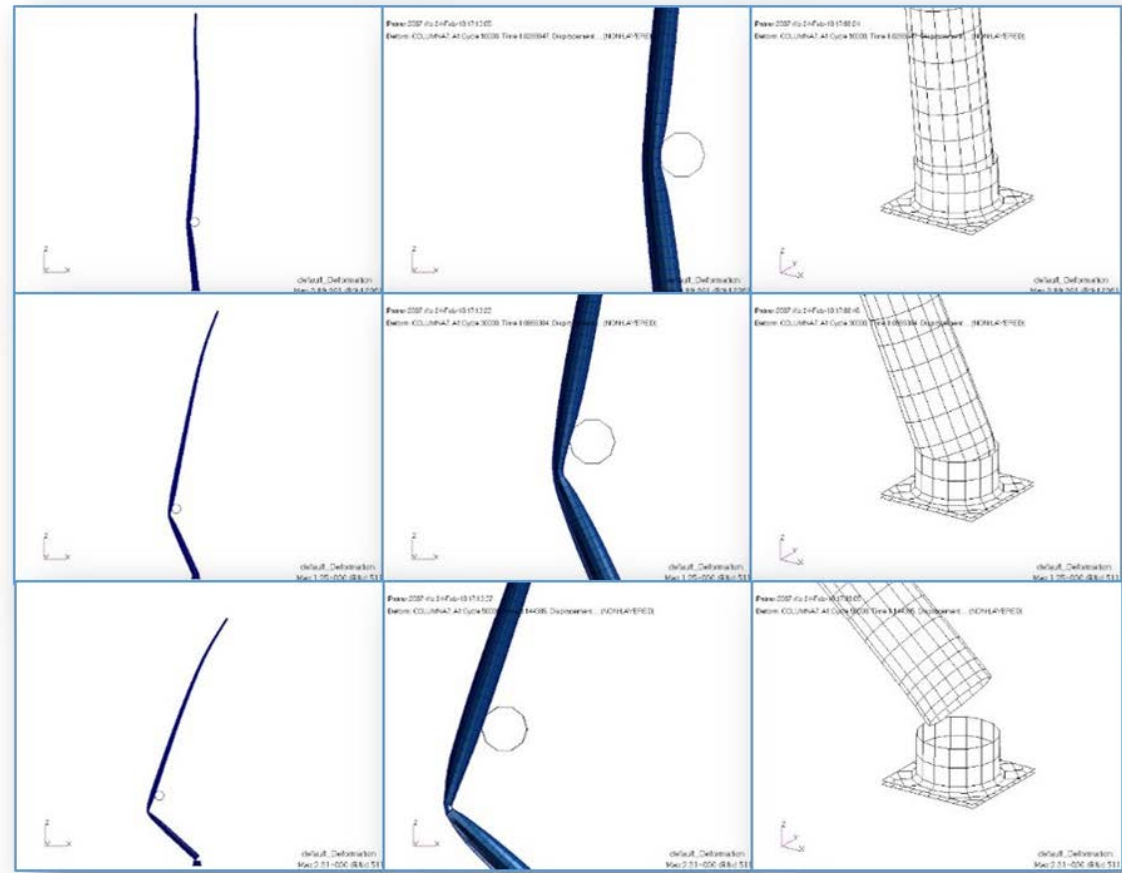

Figura 3.7: Impacto a 50Km/h. Detalles de la colisión en base y columna

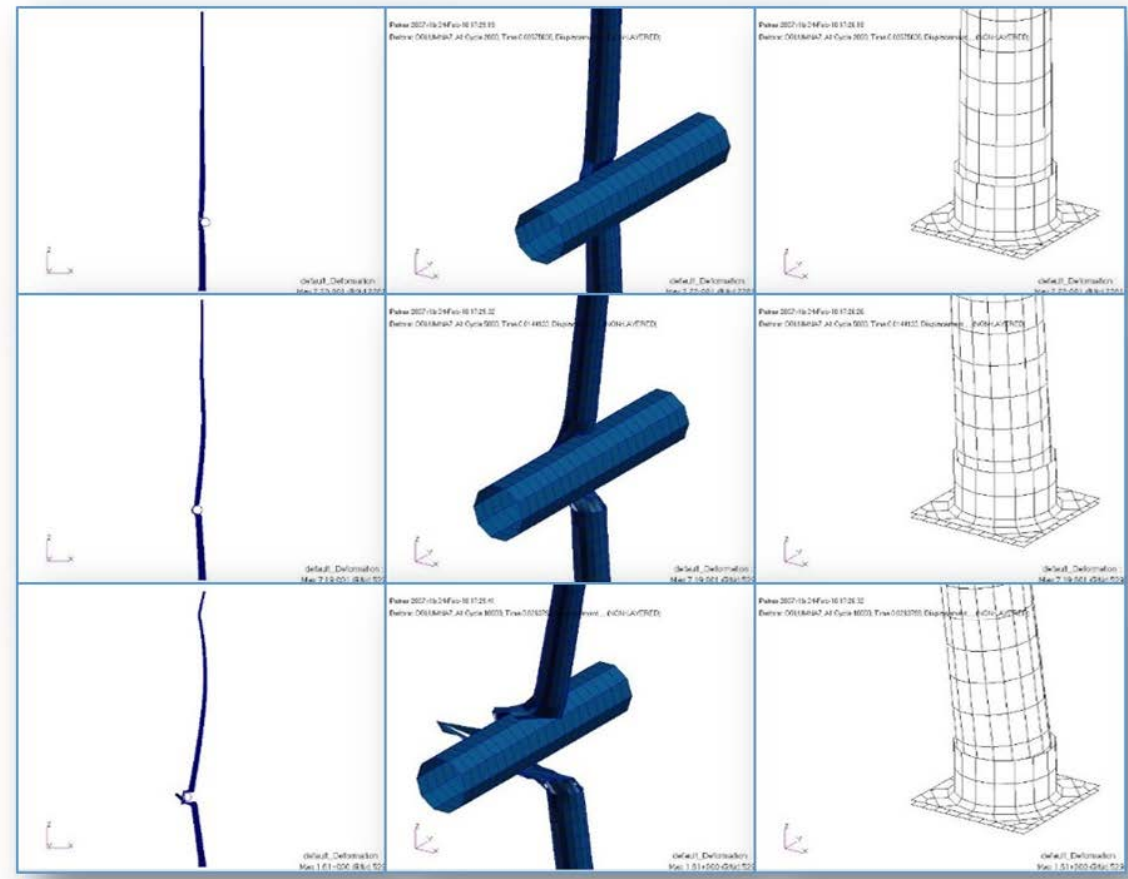

Figura 3.8: Impacto a 140Km/h. Detalles de la colisión en base y columna 


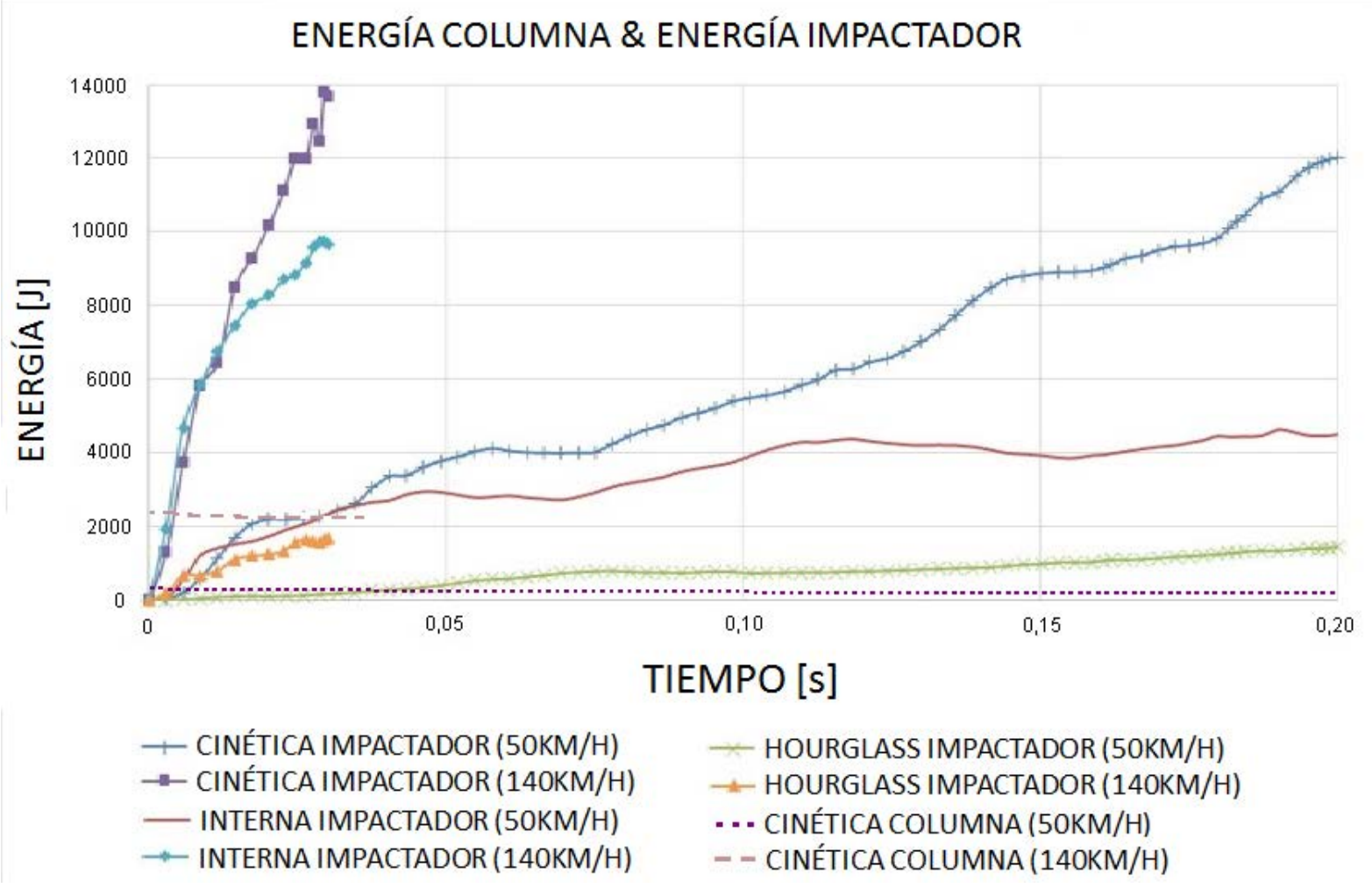

Figura 3.9: Energías de la columna e impactador

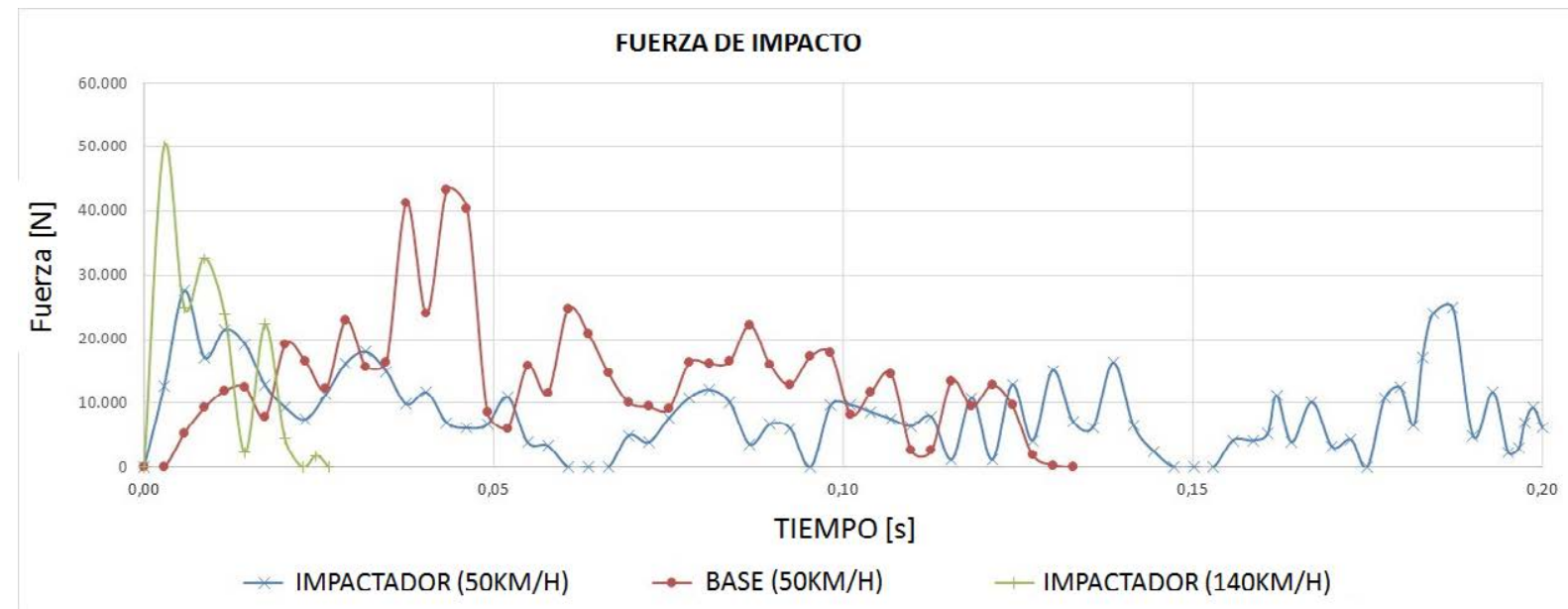

Figura 3.10: Fuerza del impacto

\subsection{Incidentes de vuelo; pérdida de pala de un avión de trans- porte}

En este caso se va a introducir un posible incidente en vuelo, la pérdida de pala de un turbohélice. Se va a estudiar el fenómeno tanto desde un punto de vista histórico y cronológico investigando el número de incidentes ocurridos como desde el punto de vista de cómo se ha 
tratado en las referencias. También se va a explicar el incidente en sí. Para acabar con el apartado se mostrará un ejemplo sencillo pero representativo para ya pasar a estudiar el fenómeno en profundidad en los siguientes capítulos.

\subsubsection{Análisis del fenómeno de la pérdida de pala}

Como ya se ha mencionado la fiabilidad es uno de los mayores problemas y preocupaciones en el diseño de aeronaves y su operación. Los motores de los aviones se operan durante miles de horas hasta que se inspeccionan, esto sucede periódicamente. La tasa de incidencias debidas a fallo de motor y/o una respuesta inadecuada de la tripulación se ha mantenido esencialmente constante durante muchos años como se indica en el reportaje Sallee y Gibbons (1998).

Varios tipos de sucesos son los que pueden producir un fallo de motor: impacto de pájaro, granizo o hielo, o con cualquier resto de la pista de despegue en las palas o buje de la hélice. De hecho, en el caso muy poco frecuente de fallo de motor, el diseño del sistema reacciona para que este único fallo no haga peligrar el funcionamiento de la aeronave. Cada sistema motor se puede aislar de los demás y configurar para que si es necesario se paren cada uno de ellos individualmente. Por lo tanto, un motor inoperativo no constituye un problema de seguridad, las aeronaves están diseñadas para volar y operar en esas condiciones.

Los fallos más graves se producen cuando es imposible de evitar que las vibraciones se transmitan a la estructura de la aeronave. Son las siguientes: desequilibrio en la unión de la hélice, fallo del soporte, o en particular, una grieta en el buje de la hélice que pueda producir una pérdida de la pala. La bancada del motor (EMS) debe estar diseñada para absorber estas vibraciones y, en caso necesario, desprender el motor de la estructura antes de que pueda producir un fallo catastrófico. En las bases de datos de seguridad aérea ASN (Última actualización: 12 de abril de 2015, accedido el 12 de abril de 2015) hay recogidos más de 470 incidentes de motor desde 1919 (pérdida de potencia, incendio, apagado, parada o mal funcionamiento del motor, inversión de potencia, simulación de fallo de motor, etc,), incluyendo más de 50 incidentes con fallos incontrolables del motor, otros 48 incidentes con separaciones de motor y al menos 30 incidentes con pérdida de pala desde 1943. El último incidente fue el 1 de noviembre de 2014 donde un avión Havilland DH-114 Heron tuvo que hacer un aterrizaje de emergencia tras una pérdida de motor turbohélice que al separarse golpeó otro motor causándole daños considerables como está recogido en Olguin (Última actualización: 5 de enero de 2015, accedido el 12 abril 12 de 2015).

Un análisis en detalle de este tipo de fallos mejorará los futuros diseños, sin embargo, se trata de fenómenos altamente dinámicos y no-lineales lo que les hace problemas muy difíciles de estudiar. Pérez et al. (2011), por ejemplo, explica como aproximarse a fenómenos no lineales en la dinámica estructural. Por ahora las entidades certificadoras tienen en consideración estos incidentes mediante modificaciones de las cargas de diseño o minimizando los riesgos para la 
estructura. A este respecto se han realizado muchos trabajos de investigación: Kinnersley y Roelen (2007) contribuye en su trabajo al diseño para evitar los accidentes, los requerimientos para la contención del motor están recogidos en la FAR Part 33 y son puestos de manifiesto en Bin (2012), el impacto de pájaro o hielo es analizado desde un punto de vista certificador en Guida et al. (2013) y Bin y Zhou (2011).

Como se ha señalado algunos incidentes derivan en un fallo incontrolable del motor como puede ser un impacto de un fan de turbina o compresor. La alta energía de rotación del mecanismo producida por el fallo tiene consecuencias para la estructura de la aeronave. Este fenómeno se ha estudiado por Zhang y Li (2011) y Wang et al. (2011) desde el punto de vista de las entidades certificadoras. El análisis de la probabilidad y/o riesgo de estos incidentes también lo ha estudiado Huyse y Enright (2003). Según las autoridades reguladoras, la aeronave debe soportar un fallo así sin que suponga un riesgo grave y, por lo tanto, ser capaz de completar el vuelo con éxito. A día de hoy es necesario una campaña completa de ensayos para los nuevos desarrollos de motores. Sólo en el caso de modificaciones menores de un diseño anterior se permite utilizar métodos de análisis para certificar el nuevo motor.

En este punto se plantea la simulación del fenómeno como alternativa a los costosos ensayos. La simulación de sistemas dinámicos es uno de los temas de mayor interés para la ingeniería debido a la complejidad de los sistemas que se diseñan y por el crecimiento de las velocidades de cálculo que permiten cada vez el análisis de modelos más complejos. Esto ha conllevado el desarrollo de la formulación, implementación y desarrollo comercial del método de los elementos finitos y muchas de sus variantes con el que se simplifican los sistemas continuos a un número finito de grados de libertad lo suficientemente grande como para reproducir el problema. Los problemas que se llegan a simular actualmente incluyen todo tipo de no linealidades y leyes de material que incluyen daño y rotura de elementos. Sin embargo aunque se puedan simular puede pasar que pequeñas diferencias en los parámetros de entrada desemboquen en consecuencias muy distintas. Este comportamiento unido al propio de la complejidad del sistema hace que los resultados sean a veces poco concluyentes.

\subsubsection{Fenómeno físico de la pérdida de pala}

Una pala de hélice progresa a través del aire a lo largo de una trayectoria aproximadamente helicoidal como resultado de su velocidad de avance y de rotación. Para girar la pala de la hélice, el motor ejerce un par torsor. Este par se reacciona en las secciones de la pala en dirección opuesta por las componentes de sustentación y resistencia como se muestra en la figura 3.11. Debido a las fuerzas de rotación reaccionando en el aire la velocidad de rotación se mantiene en la estela de la hélice con el mismo sentido de giro. Esta velocidad de rotación por la masa del aire es proporcional a la potencia de entrada. La suma de todos los componentes de sustentación y resistencia de las secciones de la pala en la dirección de vuelo es igual al empuje producido. La 
pala de una hélice debe estar diseñada para soportar fuerzas centrífugas muy altas. La estructura de la pala tiene que la fuerza de empuje más cualquier otra fuerza vibratoria que se genere.

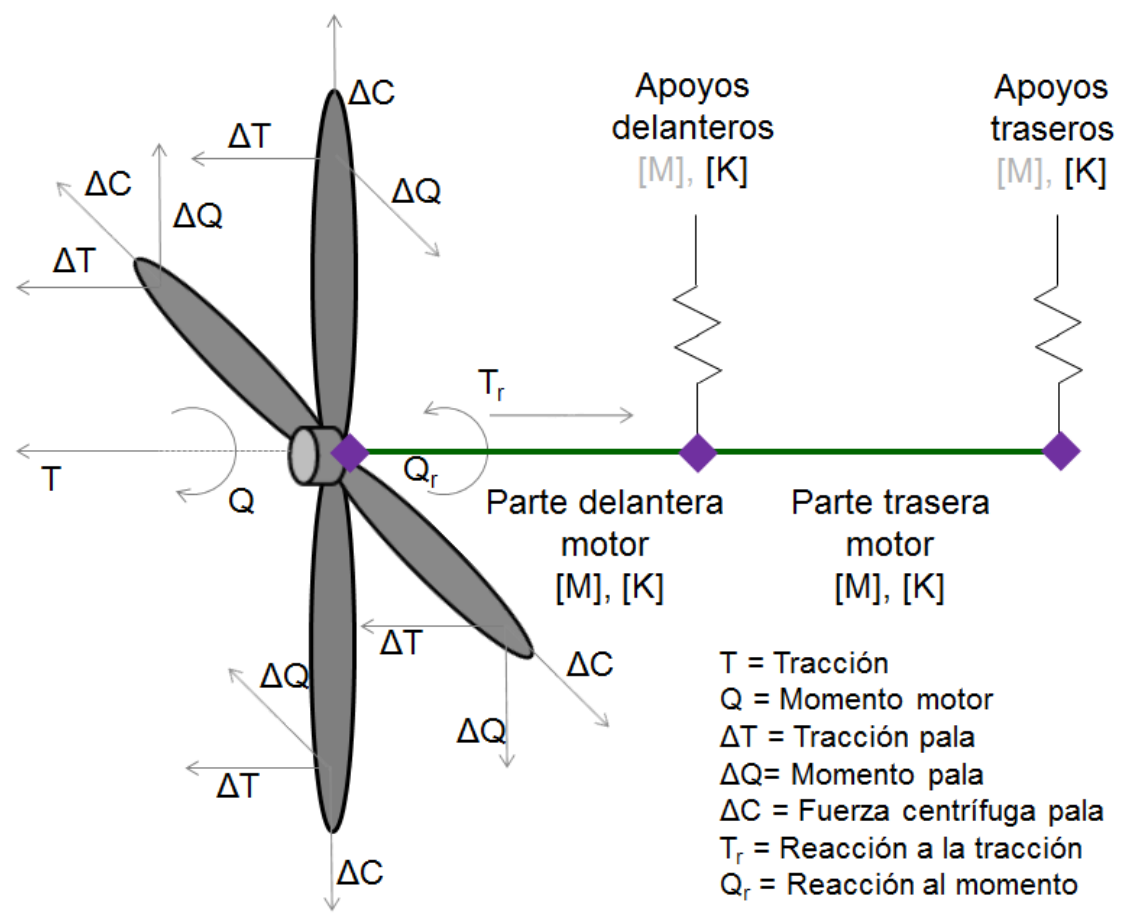

Figura 3.11: Fuerzas aerodinámicas sobre las palas y simplificación del motor y sus apoyos

Cuando se separa la pala de la hélice, muchas fuerzas que había en la parte desprendida ya no son soportadas (sustentación y resistencia, etc.) y hay fuerzas que ya no son compensadas, como la fuerza centrífuga de la pala. Estas fuerzas en este fenómeno ya han sido estudiadas y evaluadas, ver las referencias ASN (Última actualización: 12 de abril de 2015, accedido el 12 de abril de 2015) y Sinha (2013). Estos efectos dinámicos pueden causar niveles de esfuerzos muy altos, tanto en el motor como en su sistema de unión con el ala y el resto de la aeronave, como se muestra en la figura 3.11.

Esta situación es para la tripulación y, en especial, para el piloto muy similar a un daño grave en el motor o incluso su pérdida. El piloto debe estabilizar la aeronave y seguir los procedimientos recomendados para un daño grave de motor o su pérdida. Normalmente, suele consistir la puesta en banderola de la hélice y apagado del motor. No es fácil identificar la causa de la vibración si no hay alguna otra indicación. Otros parámetros del motor pueden ayudar para establecer si existe fallo en el motor.

En este fenómeno, la evaluación de los daños no puede discriminar si el daño se debe principalmente al período transitorio después de la pérdida de pala o si se debe al estado después de la estabilización. Incluso en pruebas instrumentadas, es difícil determinar el daño debido a los efectos transitorios ya que es un fenómeno muy corto. Sin embargo es obvio que se inflige daño al motor y sus componentes. Los motores turbohélice que pierden una pala comienzan rápidamente 
a vibrar con fuerza con considerables consecuencias; daños de material y fallos estructurales, y otros daños como se puede ver en Kushan et al. (2007). Normalmente sólo causa daños críticos en el motor y su sistema de apoyo ya que la unión absorbe y soporta estas cargas. Si el sistema de unión del motor (típicamente dispositivos elastoméricos) falla, el camino de carga al ala se interrumpe. Los dispositivos están diseñados con este propósito: para actuar como un fusible con el fin de reducir los riesgos de fallo catastrófico para el resto de la estructura.

\subsubsection{Ejemplo: Viga en voladizo con carga armónica seguidora}

Se plantea este ejemplo como una simplificación extrema de lo que sucede en el fenómeno de la pérdida de pala. La viga representa el motor, el empotramiento del extremo de la viga simula los apoyos del motor como se puede ver en la figura 3.12. Finalmente la carga en el otro extremo de la viga tiene la misma naturaleza que las cargas que aparecen cuando se pierde una pala.

Se somete al extremo de una viga en voladizo a una carga variable con el tiempo $P(t)=$ $P_{0} \cos (\Omega t)$ que siempre permanece perpendicular a la viga (fuerza seguidora). Los datos iniciales del problema son: viga cuadrada maciza lado $0,1 \mathrm{~m}[\mathrm{~m}]$, módulo de Young de la viga $E=70000$ [MPa], módulo de Poisson $\nu=0,33$, longitud inicial de la viga $L_{0}=10[\mathrm{~m}]$, amplitud de la fuerza $P_{0}=100000[\mathrm{~N}]$, frecuencia de la fuerza $\Omega=100[\mathrm{rad} / \mathrm{s}]$ y densidad del aluminio $\rho=2700\left[\mathrm{~kg} / \mathrm{m}^{3}\right]$. En la figura 3.13 se muestra el convenio de nomenclatura que se ha usado en
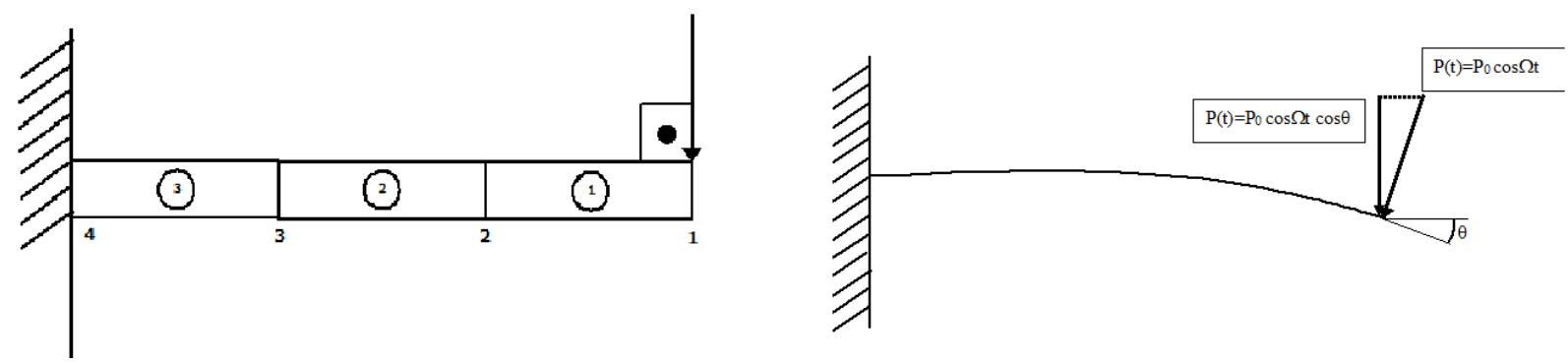

Figura 3.12: Viga en voladizo con carga armónica seguidora

el problema. No se está interesado en el movimiento del eje x por lo que se obvia la fuerza en esta dirección. Los corrimientos $v_{G}$ de la línea elástica en el plano principal xy pueden descomponerse en los $v_{G b}$ originados por las deformaciones de flexión y los $v_{G s}$ originados por las deformaciones de cortadura, que vienen definidos por las ecuaciones siguientes:

$$
\begin{aligned}
& E I_{z} \frac{d^{2} v_{G b}}{d x^{2}}=M_{z} \\
& G A_{y} \frac{d v_{G s}}{d x}=S_{y}
\end{aligned}
$$

En donde $A_{y}$ es el área reducida en cortadura en dirección del eje y, en el caso de una sección rectangular es $A_{y}=(5 / 6) b h$. Al final, la solución del problema puede expresarse como: 


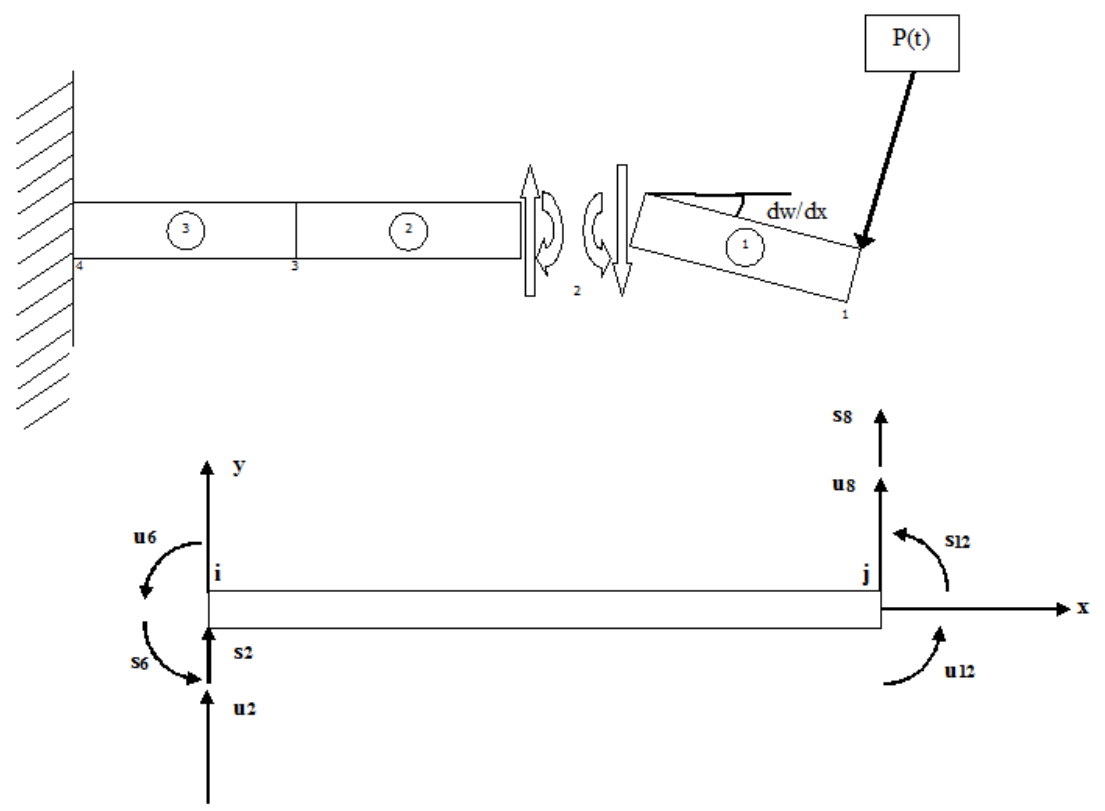

Figura 3.13: Elementos de la viga y criterio de signos

$$
\{s\}=[k]\{u\}+\left\{s_{0}\right\}
$$

Con los valores:

$$
[k]=\left(\begin{array}{cccc}
k_{2,2} & k_{2,6} & k_{2,8} & k_{2,12} \\
k_{6,2} & k_{6,6} & k_{6,8} & k_{6,12} \\
k_{8,2} & k_{8,6} & k_{8,8} & k_{8,12} \\
k_{12,2} & k_{12,6} & k_{12,8} & k_{12,12}
\end{array}\right)=\left(\begin{array}{cccc}
\frac{12 E I_{z}}{L^{3}\left(1+\Phi_{y}\right)} & \frac{6 E I_{z}}{L^{2}\left(1+\Phi_{y}\right)} & \frac{12 E I_{z}}{L^{3}\left(1+\Phi_{y}\right)} & \frac{6 E I_{z}}{L^{2}\left(1+\Phi_{y}\right)} \\
\frac{6 E I_{z}}{L^{2}\left(1+\Phi_{y}\right)} & \frac{E I_{z}\left(4+\Phi_{y}\right)}{L\left(1+\Phi_{y}\right)} & \frac{6 E I_{z}}{L^{2}\left(1+\Phi_{y}\right)} & \frac{E I_{z}\left(1+\Phi_{y}\right)}{L\left(1+\Phi_{y}\right)} \\
\frac{12 E I_{z}}{L^{3}\left(1+\Phi_{y}\right)} & \frac{6 E I_{z}}{L^{2}\left(1+\Phi_{y}\right)} & \frac{12 E I_{z}}{L^{3}\left(1+\Phi_{y}\right)} & \frac{6 E I_{z}}{L^{2}\left(1+\Phi_{y}\right)} \\
\frac{6 E I_{z}}{L^{2}\left(1+\Phi_{y}\right)} & \frac{E I_{z}\left(2-\Phi_{y}\right)}{L\left(1+\Phi_{y}\right)} & \frac{6 E I_{z}}{L^{2}\left(1+\Phi_{y}\right)} & \frac{E I_{z}\left(4+\Phi_{y}\right)}{L\left(1+\Phi_{y}\right)}
\end{array}\right)
$$

En donde $\Phi_{y}=\frac{12 E I_{z}}{L^{2} G A_{z}}$. Partiendo de la ecuación general del movimiento 2.3 quedaría:

$$
\left(\begin{array}{cccc}
m & 0 & 0 & 0 \\
0 & I & 0 & 0 \\
0 & 0 & m & 0 \\
0 & 0 & 0 & I
\end{array}\right)\left\{\begin{array}{c}
\ddot{u_{2}} \\
\ddot{u_{6}} \\
\ddot{u_{8}} \\
\ddot{u_{12}}
\end{array}\right\}+[k]\left\{\begin{array}{c}
u_{2} \\
u_{6} \\
u_{8} \\
u_{12}
\end{array}\right\}=\left\{\begin{array}{c}
0 \\
0 \\
-P \cos \frac{d w}{d x}(L) \\
u_{12}
\end{array}\right\}
$$

Se ha definido la matriz de rigidez de la viga en función de E, I, L, A, G. La matriz de rigidez será constante para todos los elementos. Se integran las ecuaciones con un esquema implícito $\beta$-Newmark. Se calculan los momentos de inercia rotacionales, siendo en los extremos $I_{r o t}=(1 / 3) m l^{2}$ y en los puntos intermedios $I_{r o t}=(1 / 12) m l^{2}$. También se calculan las reacciones $R_{2}$ y $R_{6}$. Los resultados se pueden ver en la figura 3.14 para un $\Delta t=10^{-4} \mathrm{~s}$. Se puede observar en los resultados que la estructura vibra a la frecuencia natural de la viga $\sim 1 \mathrm{~Hz}$, además de la frecuencia de la fuerza exterior. Se adjuntan también en 3.14 los valores obtenidos en MSC.Nastran que es un software de análisis estructural multidisciplinar que utiliza métodos 

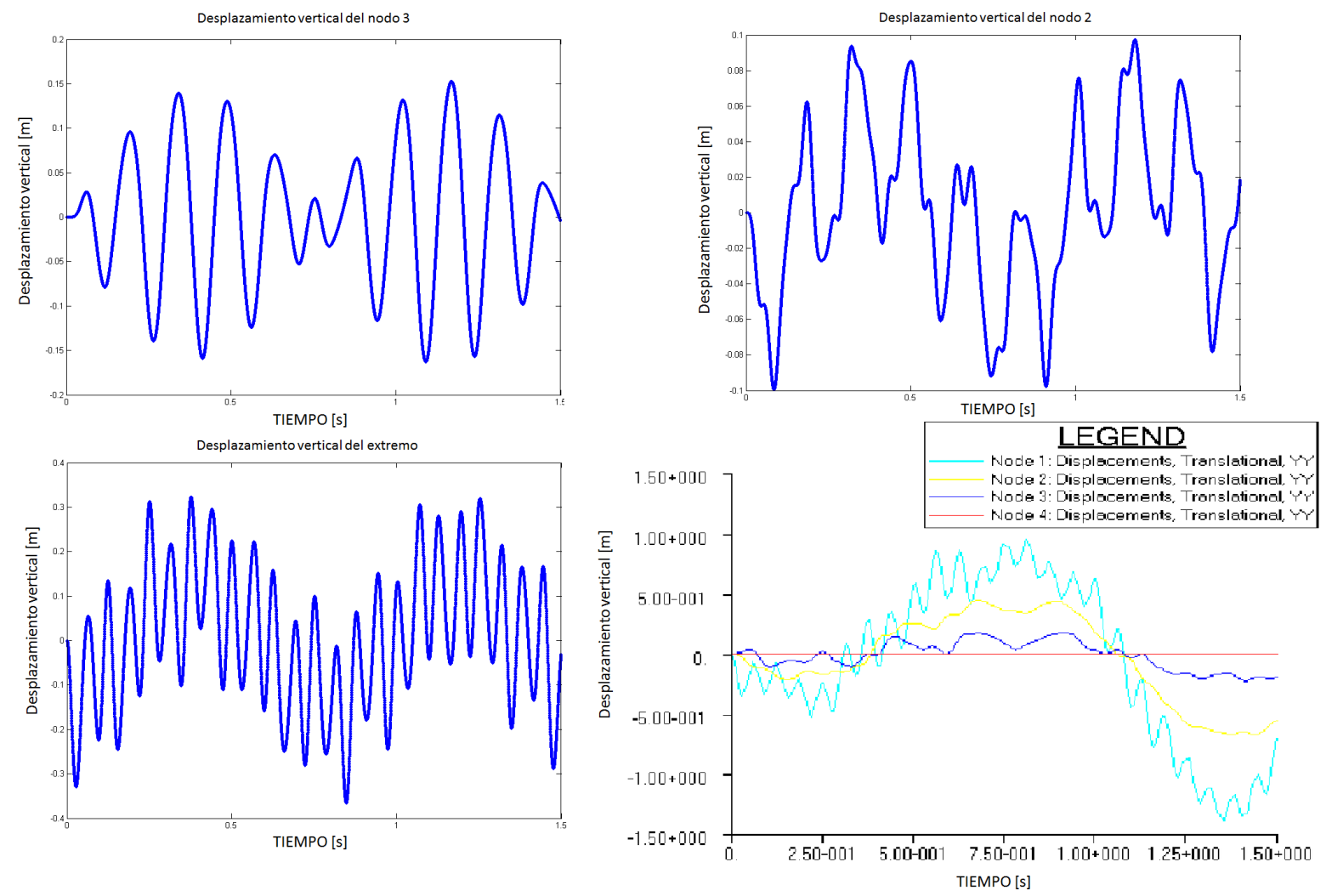

Figura 3.14: Desplazamiento nodos en función del tiempo

de resolución implícitos para realizar análisis estáticos, dinámicos, térmicos,... El problema introducido en MSC.Nastran es un problema muy similar (viga en voladizo con fuerza senoidal a $16 \mathrm{~Hz}$ en el extremo). 


\section{ANÁlisis DETERMINISTA DE LA PÉRDIDA DE PALA}

\section{Índice}

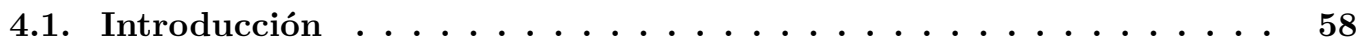

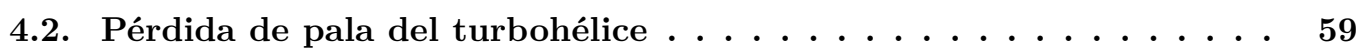

4.3. Descripción del modelo . . . . . . . . . . . . . . . . . 61

4.4. Modelo FEM . . . . . . . . . . . . . . . . . . 62

4.4.1. Cargas . . . . . . . . . . . . . . . . . . . . . . . 64

4.4.2. Unión bancada-ala . . . . . . . . . . . . . . . . . . . 65

4.4.3. Unión motor-bancada . . . . . . . . . . . . . . . . . . . . . . 65

4.4.3.1. Modelo elastomérico . . . . . . . . . . . . . . . . . . . 66

4.4.4. Consideraciones de los análisis explícitos . . . . . . . . . . . . 68

4.4.4.1. Chequeos másicos y energéticos . . . . . . . . . . . 69

4.4.4.2. Chequeo de modos propios . . . . . . . . . . . . 70

4.4.5. Casos de carga analizados . . . . . . . . . . . . . . . . 71

4.4.5.1. Posición angular . . . . . . . . . . . . . . . . . 72

4.4.5.2. Puntos de la envolvente de vuelo . . . . . . . . . . . . . 73

4.4.5.3. Sensibilidad a las $\mathrm{rpm} \ldots \ldots \ldots$. . . . . . . . . 73

4.4.5.4. Sensibilidad a la rigidez de la unión bancada-ala . . . . . . . . 74

4.4.5.5. Momento y forma de introducción de las fuerzas . . . . . . . 74

4.4.5.6. Tamaño de la pala perdida . . . . . . . . . . . 75

4.4.5.7. Amortiguamiento estructural . . . . . . . . . . . . 76

4.4.5.8. Flexibilidad del ala . . . . . . . . . . . . . 76

4.4.6. Resultados de los casos de carga . . . . . . . . . . . . . . . 77

4.4.6.1. Resultados obtenidos . . . . . . . . . . . . . . . . . 79

4.4.6.2. Resumen resultados por grupos de caso de carga . . . . . . . 88 


\subsection{Introducción}

Los aviones modernos son, sin ningún tipo de dudas muy fiables, sobre todo teniendo en cuenta el escaso número de incidentes que tienen. En particular, los motores de aviación son operados miles de horas antes de pasar por sus programas de revisiones e inspecciones. Por otra parte, esas inspecciones son un gasto de tiempo y de dinero para las compañías como se pone de manifiesto en Reményi y Staudacher (2012). Sin embargo, hay en alguna rara ocasión que ocurre un mal funcionamiento del motor. Cada motor se debe instalar de manera que un mal funcionamiento de uno de ellos no ponga en peligro la seguridad del vuelo.

Además, existen unos límites estadísticos en los cuales la probabilidad del fallo es tan extremadamente remota que no se considera. Cada motor debe estar configurado y aislado de los otros para permitir la operación de la aeronave en cualquier circunstancia y debe haber medios para parar cualquier motor de forma individual en caso de un mal funcionamiento. Un motor inoperativo no constituye un problema de seguridad ya que los aviones con varios motores están diseñados para volar en esas circunstancias. De hecho, la mayoría de las paradas del motor pasan desapercibidas por los pasajeros de vuelos comerciales y pueden ser debidos a un impacto de pájaro, granizo o hielo, el impacto de otros restos o incluso por fenómenos naturales, tales como la formación de hielo en la pala de la hélice o el buje. Estos episodios pueden causar daños a la estructura del motor y de la aeronave. Un análisis en detalle de estos fenómenos pueden mejorar los diseños de futuras aeronaves. Los análisis no son sencillos, sobre todo debido a las características no lineales de muchos de los sucesos que se producen en ellos; Pérez et al. (2011) explica cómo tratar fenómenos no lineales en la dinámica estructural.

Este tipo de fenómenos se tienen en cuenta a través de la modificación de las cargas de diseño, reduciendo al mínimo el riesgo para la estructura, y pudiendo así convencer a las entidades certificadoras. Kinnersley y Roelen (2007) estudia la proporción de accidentes en cuyas causas se encontraba el diseño de la estructura, Bin (2012) explica los puntos clave en lo que respecta a la contención de las partes dañadas en la estructura del motor cumpliendo la normativa FAR 33, Guida et al. (2013) con respecto al impacto de pájaro y Bin y Zhou (2011) con respecto al hielo analizan el impacto con la estructura desde el punto de vista de la certificación.

Hay incidentes que implican consecuencias para la estructura de la aeronave tales como la pérdida de una pala de la etapa de compresión de un motor, o la propia contención del motor ante fallos mecánicos, Zhang y Li (2011) y Wang et al. (2011) los estudian desde el punto de vista de la certificación. Hay artículos que analizan la probabilidad o riesgo de estos incidentes como Huyse y Enright (2003). Según las autoridades reguladoras, el avión debe ser capaz de completar con éxito el vuelo aun sufriendo el fallo de un motor sin que esto suponga un peligro importante para la aeronave. Para los nuevos desarrollos las autoridades siguen requiriendo campañas completas de ensayos de motor para su certificación. La certificación sin nuevos ensayos sólo se permite en caso de cambios menores y siempre partiendo de un diseño certificado de motor anterior. 
Sin embargo, la progresión de las investigaciones y la mejora de las herramientas de simulación hacen que se pueda ahorrar tiempo y dinero en un futuro próximo.

Uno de los fallos más graves y peligrosos es la pérdida de pala de un turbohélice, como se puede ver en Hall et al. (1996) y Board (1993). En algunos casos es imposible evitar las vibraciones que provienen de grietas en el buje de la hélice que pueden incluso derivar en la pérdida de pala de la hélice, la pérdida del control, y posibles daños en el avión. Tanto el daño a la estructura como a los sistemas por el impacto por la pérdida de pala o por el desequilibrio que crea debe tenerse en cuenta Hickey (2000). En este capítulo se estudia la respuesta de la estructura después de una pérdida de pala de la hélice mediante el método de elementos finitos siguiendo las pautas ya marcadas en Kushan et al. (2007) y Stallone et al. (1982) donde se investigan los motivos del fallo de la pala y donde se desarrolla un método analítico para predecir la respuesta no lineal transitoria de un sistema motor de avión completo que sufre la pérdida de una pala.

La respuesta de la estructura después de una pérdida de pala de la hélice varía radicalmente con las condiciones de vuelo, la frecuencia de la hélice, el tamaño de la pala perdida, la posición angular donde se produce la pérdida y la manera en cómo se produce la pérdida ya que esta pérdida se puede producir instantáneamente o de forma gradual. Diken y Alnefaie (2011) analizan algunos de estos efectos en un rotor flexible. Además, la flexibilidad del ala, la rigidez de sus apoyos, el amortiguamiento estructural también son factores determinantes del fenómeno, Dygadlo et al. (2003) y Mello (1998) analizan los efectos de estos parámetros en un helicóptero.

Este capítulo está organizado de la siguiente manera. La sección 4.2 explica el fenómeno de la pérdida de pala. La sección 4.3 y 4.4 describen el modelo utilizado para simular el suceso, la filosofía, las decisiones tomadas y los resultados obtenidos. Finalmente la sección 6.1.1 resume las conclusiones que se dirimen de los resultados.

\subsection{Pérdida de pala del turbohélice}

En este caso las palas rotan cerca de 840 rpm, similar a cualquier avión militar turbo-hélice por lo que están diseñadas para soportar cargas centrífugas muy altas, ver la figura 4.1. Además también deben soportar cualquier otra fuerza que se genere, como las causadas en una ráfaga. Los motores turbohélices tienen un menor número de partes móviles y cuentan con una mayor fiabilidad debido la suavidad de su funcionamiento sin vibraciones. Los turbohélices son generalmente más eficientes en altitudes de entre 20.000 y 30.000 pies y a velocidades medias, y tiene un mayor tiempo entre revisiones que otro tipo de aeronaves. Por el contrario, la probabilidad de que pueda perder una pala por un crecimiento de grieta que pasó desapercibida crece por este último motivo. Esta situación se estudia en detalle en Staff (1997), en este caso para un avión ver en la figura 4.1 donde una grieta creció y la pala de la hélice se separó completamente. 


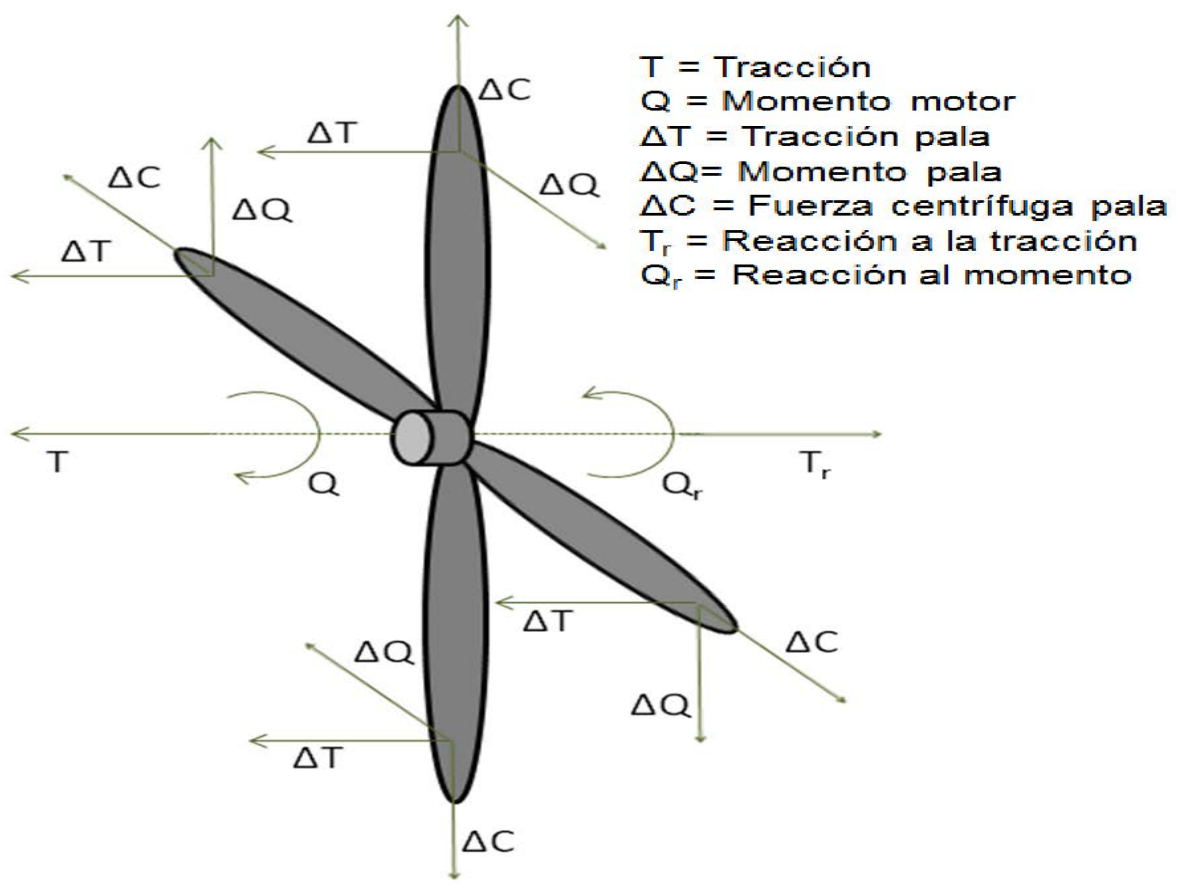

Figura 4.1: Fuerzas aerodinámicas sobre las palas de la hélice

Cuando se separa la pala de la hélice, desaparecen muchas fuerzas (sustentación y resistencia del trozo desprendido) y otras dejan de compensarse, como la fuerza centrífuga de la pala (proporcional a la masa y al cuadrado de la velocidad rotacional), Sinha (2013) evalúa estas fuerzas para el caso de carga de pérdida de un rotor de un turbofan. Estos efectos dinámicos producen altos niveles de esfuerzos en el motor, sus apoyos, el ala y el resto del avión como se muestra en la figura 4.2 .

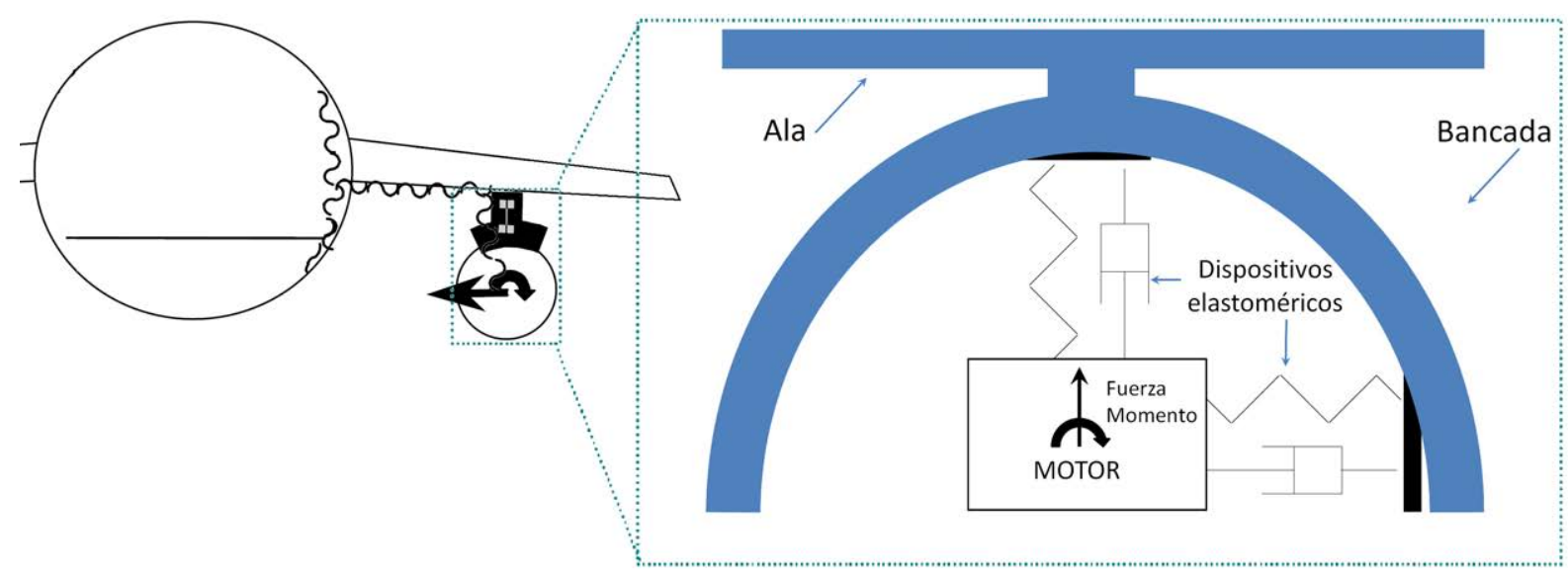

Figura 4.2: Vibraciones en la aeronave (izquierda) y detalle del sistema de anclaje del motor (derecha) 
Como se ve más adelante el fenómeno de pérdida de pala se analiza y simula mediante el método de elementos finitos. También estudia la influencia del tamaño de pala perdida. Durante la pérdida de pala algunas partes del sistema de apoyo del motor, la bancada o . Engine Mounting System"(EMS), se rompen (especialmente los dispositivos elastoméricos), por lo tanto sus propiedades son críticas y se tienen en cuenta mediante su rigidez y resistencia. Además las distintas roturas producen una secuencia de fallo que es estudiada en profundidad. Los parámetros que se han considerado importantes son el tamaño de pala perdida, la rigidez de la unión ala-EMS, la flexibilidad del ala, la posición angular en la que se produce la pérdida de pala, la condición de vuelo mediante la frecuencia de la hélice, altitud y velocidad y el amortiguamiento estructural. La simulación tiene en cuenta el efecto de la variación de estos parámetros y sus efectos en el comportamiento estructural. Por lo tanto, las condiciones de contorno (ala-EMS), el comportamiento no lineal (geométrico y de material) y las condiciones de fallo se consideran en la simulación y en cualquier parte de la estructura.

\subsection{Descripción del modelo}

Para analizar y simular el fenómeno de la pérdida de pala se ha escogido un ejemplo representativo, un avión militar pesado de transporte con un turbo-hélice de aproximadamente $11000 \mathrm{HP}$. Cuatro de estos motores proporcionan a un avión $135000 \mathrm{~kg}$ de peso de despegue, casi $225 \mathrm{~m} / \mathrm{s}$ de velocidad de crucero y un radio de acción de $3200 \mathrm{~km}$. El motor está equipado con reductor y una hélice de 8 palas de material compuesto. Las partes del avión que se van a modelizar van desde la hélice pasando por el motor, la unión a la bancada hasta la unión de la bancada con el ala.

Como se ha mencionado antes, la estructura está diseñada para soportar una serie de cargas a lo largo de su vida útil. Sin embargo, una pérdida de pala implica niveles de esfuerzos muy altos que pueden derivar en un fallo de alguna parte estructural. El fallo del EMS puede evitar el fallo crítico del resto de la estructura. La figura 4.2 muestra el esquema del modelo simplificado. Los aspectos más importantes que el modelo incluye son el comportamiento dinámico del motor y su apoyo, en particular su masa e inercia. Además, los modos propios del modelo deben ser lo más parecidos posible a los modos de la estructura real. Otro importante aspecto a tener en cuenta es el comportamiento de los apoyos del motor al ala ya que los resultados dependen enormemente de sus propiedades. Se han estudiado varios valores de rigidez, desde valores muy flexibles hasta muy rígidos. La figura 4.3 muestra el diseño del EMS.

La figura 4.4 muestra las propiedades de rigidez de los apoyos. Es esencial una optimización del material elastomérico como se pone de relieve en Zheng-Dong y Chang (2004). El problema no lineal debe tener en cuenta las características de la rotura de estos dispositivos elastoméricos así como de su rigidez no lineal. Es más, el criterio de fallo de estas partes determinan el comportamiento de todo el sistema. Cambios en los apoyos debidos a una rotura o 

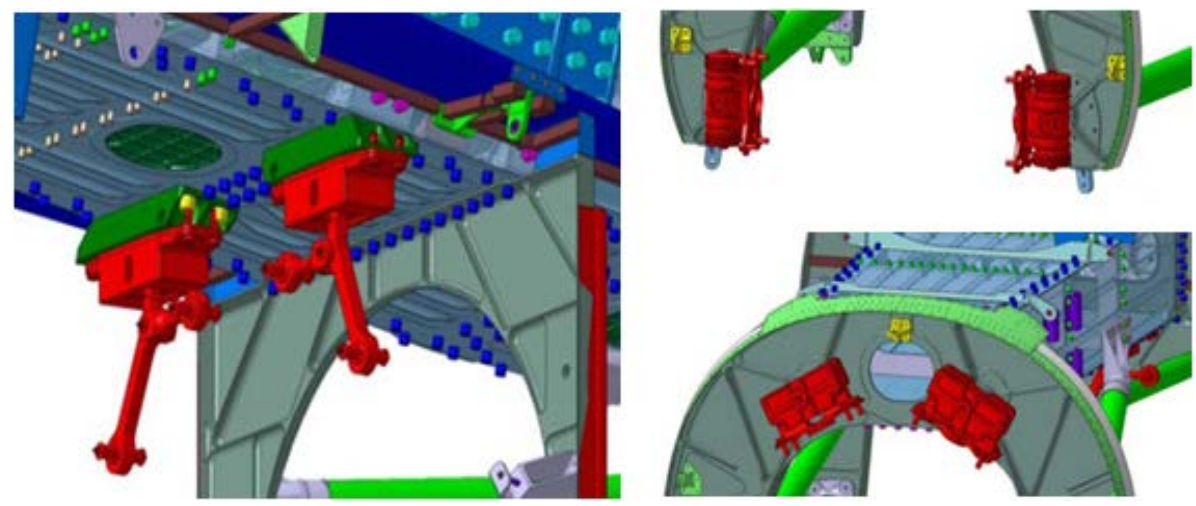

Figura 4.3: Sistema de anclaje del motor (EMS). Apoyos traseros o rear mounts"(izquierda) y superiores delanteros o "top mount"(abajo derecha) y laterales delanteros o "side mount"(arriba derecha)

fallo modifican la dinámica del sistema al modificar las relaciones de rigidez entre elementos y, como consecuencia, la secuencia de fallo de los dispositivos elastoméricos.

Por lo tanto, se subdivide el modelo haciendo mucho hincapié en las propiedades de las uniones. En Stallone et al. (1982) se trabaja con un modelo muy simplificado de motor y sistema de apoyo. La subdivisión más común es la hélice, motor principal, sistema de unión del motor a la bancada, la bancada, unión de la bancada al ala y el propio ala. El modelo y sus características se pueden ver en 4.2. Entonces si se reproducen correctamente las propiedades de la unión al ala, las propiedades de rigidez y másicas del motor y las propiedades de rigidez y amortiguamiento del sistema de apoyo del motor está bien reproducido el modelo y es capaz de simular con detalle el comportamiento del fenómeno.

\subsection{Modelo FEM}

Los apoyos y el motor se simulan mediante el método de elementos finitos conocido como MSC.Dytran, ver MSC (2005). El pre-solver estructural utilizado es MSC.Patran, que se utiliza con el solver explícito mencionado. Como se ha comentado en el apartado 2.3.1 los solvers explícitos se utilizan en el caso de simular fenómenos de corta duración temporal como impactos o choques donde la propagación de las ondas y el comportamiento dinámico son importantes. El mayor de los desafíos en este tipo de solvers suele ser el gran número de pasos de integración que se necesitan debido al pequeño tamaño de cada uno de ellos, lo mismo pasa con el tiempo CPU para correr un caso que se suele volver impracticable. El apartado 4.4.4 trata este tema en profundidad.

Respecto al motor, la característica fundamental a reproducir es la masa. En la figura 4.6 se puede ver como se ha modelizado utilizando elementos viga (BAR2) y elementos masa (POINT1) que permiten adecuar el comportamiento dinámico. El modelo de la bancada con- 

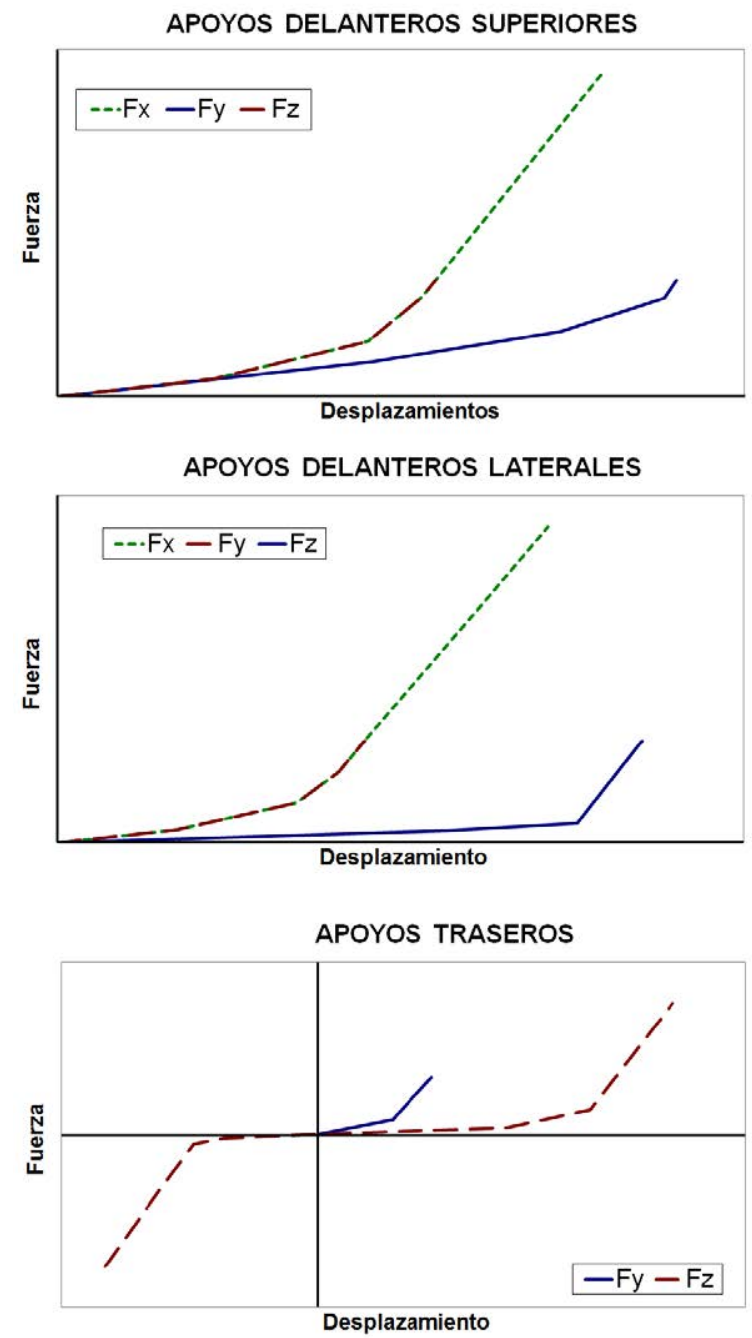

Figura 4.4: Propiedades elastoméricas

siste en elementos placa (TRIA3 y CQUAD4) y elementos viga (BAR2) que también permiten reproducir con precisión el comportamiento estructural (ver la figura 4.5).

\begin{tabular}{|c|c|c|c|c|c|c|}
\hline MATERIALES & Vigas del motor & $\begin{array}{c}\text { Elementos de } \\
\text { contorno }\end{array}$ & $\begin{array}{c}\text { Vigas de la } \\
\text { bancada }\end{array}$ & $\begin{array}{c}\text { Placas de la } \\
\text { bancada }\end{array}$ & Vigas del motor & Vigas del motor \\
\hline$E[\mathrm{GPa}]$ & 204 & 115.3 & 78.1 & 108.3 & 198.5 & 34.8 \\
\hline $\mathrm{G}[\mathrm{GPa}]$ & 77.7 & 44 & 29.7 & 41.2 & 77 & 13.4 \\
\hline$\rho\left[\mathrm{kg} / \mathrm{m}^{3}\right]$ & 200 & 60 & 1894 & 7980 & 200 & 75 \\
\hline Plasticidad & NO & YES & YES & YES & NO & NO \\
\hline
\end{tabular}

Figura 4.5: Propiedades elásticas de los distintos materiales del modelo 


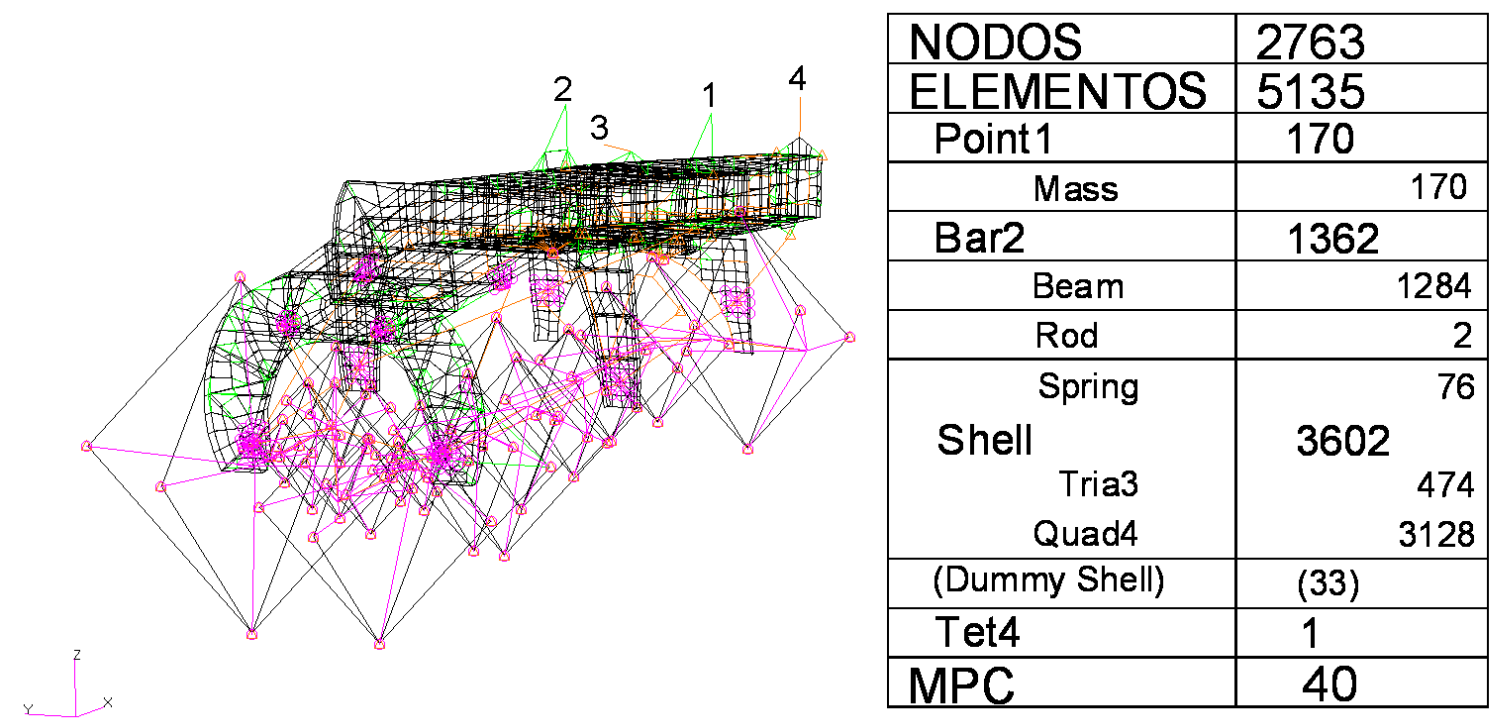

Figura 4.6: Modelo FEM y condiciones de contorno (unión bancada-ala puntos 1-4)

Como se esperan niveles de esfuerzos elevados en ciertas partes de la estructura situados mayormente en la bancada es necesario dotar al modelo de un comportamiento no lineal y se ha proporcionado un comportamiento lineal al material del motor y lineal y no lineal a la bancada (ver figura 4.7), por lo que parte de la energía absorbida por el modelo se utilizará en plastificar el material de la bancada. Se ha utilizado un criterio perfectamente plástico para el material de los elementos 1D y 2D de la bancada. Los elementos entre la bancada y el ala reproducen la rigidez de la unión, se describen más en detalle en el apartado 4.4.2. Para terminar, el ala se ha sustituido por un apoyo, ya que el objetivo es determinar las fuerzas que llegarían al ala durante la simulación. También cabe destacar que se utilizan varias Multi Point Constrain (MPC) en el modelo. Las propiedades de éstos y otros elementos se pueden consultar en la referencia MSC (2005).

\subsubsection{Cargas}

La pérdida de pala introduce un desequilibrio que se traduce en una fuerza de rotación y un momento en el buje de la hélice (ver figura 4.1) como se ha explicado en detalle en el apartado 3.3.1.1. Las fuerzas son debidas principalmente a la fuerza centrípeta que ya no es compensada del trozo de pala que se ha desprendido. Lo mismo pasa con el momento principal, que es debido a la fuerza de sustentación del trozo de pala desprendido por el brazo. Ambos dependen de la frecuencia de la hélice. Por lo tanto, las cargas consideradas se pueden escribir como; $F_{y}=-F_{c} \cos (\omega t), F_{z}=-F_{c} \sin (\omega t), M_{y}=M_{o o p} \sin (\omega t)$ y $M_{z}=-M_{\text {oop }} \cos (\omega t)$. 


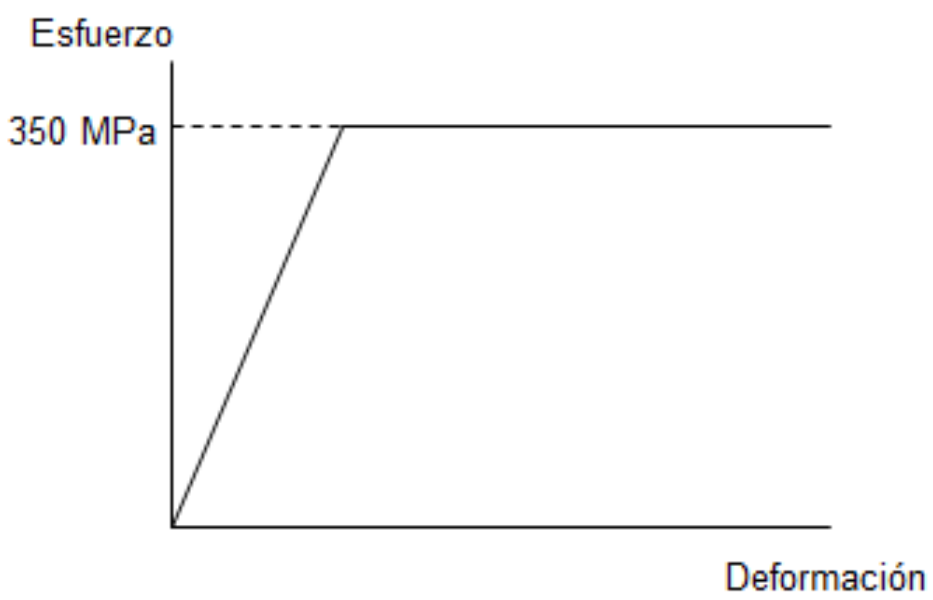

Figura 4.7: Modelo de material perfectamente plástico

\subsubsection{Unión bancada-ala}

La unión entre el ala y la bancada es la última barrera antes de que las fuerzas lleguen al ala. En el modelo se simula mediante el apoyo de cuatro nodos empotrados y elementos rígidos como se pueden ver en la figura 4.6; el número 1 apoyado en los ejes XZ, 2 en ejes XZ, 3 en el eje Y y 4 en el eje Z. Se estudia la influencia de la rigidez de estos elementos en el comportamiento estructural del sistema. La simulación puede producir resultados muy distintos si esta unión es rígida o flexible. En una unión rígida no hay desplazamiento relativo y las fuerzas se transmiten directamente mientras que en una unión flexible sí que aparece un movimiento relativo. Se varía su módulo de rigidez desde su valor nominal, ratio 1 , a un valor mucho menos rígido, ratio 0.01

\subsubsection{Unión motor-bancada}

La unión entre la bancada y el motor se realiza mediante seis apoyos por motor como se ilustra en la figura 4.3. Este diseño de unión está muy extendido en este tipo de aeronaves y se viene utilizando desde hace mucho tiempo, como se puede ver en Stallone et al. (1982). El sistema no está estáticamente determinado: hay dos apoyos laterales (side mounts) que soportan parte del empuje y la carga lateral y una parte importante del par motor, dos apoyos superiores (top mounts) que soportan otra parte del empuje, y dos apoyos traseros (rear mounts) que soportan carga lateral y vertical.

Como se ha mencionado antes, el material utilizado para la unión es de tipo elastomérico debido al comportamiento viscoelástico, normalmente con un módulo de Young bajo y un alto límite elástico. Esto permite que el elastómero absorba gran cantidad de energía antes de romper. Existen innumerables aplicaciones en las que se utiliza este tipo de material y este es uno de los casos en donde se requiere unas características de material de altas prestaciones. En Boyce y Arruda (2000) se muestran muchos modelos constitutivos de material para estos materiales. 
En el presente estudio se considera que el elastómero trabaja linealmente en cortadura y no linealmente en compresión-tensión.

\subsubsection{Modelo elastomérico}

En un modelo FEM la manera más sencilla de representar un elastómero es mediante muelles lineales (3 traslacionales y si son necesarios 3 rotacionales). Sin embargo, este modelo no puede reproducir las propiedades que se muestran en la figura 4.4 directamente por limitaciones en el software de MSC.Dytran. En este caso es necesario introducir un comportamiento no lineal en cada eje. Esto se consigue utilizando 3 muelles no lineales que proporcionan las propiedades requeridas. El problema es que no se puede introducir fallo para este tipo de elementos. Consecuentemente se introdujeron unos muelles lineales con fallo en serie con 3 muelles no lineales. Este esquema reproduce las propiedades de la figura 4.4 hasta el fallo.

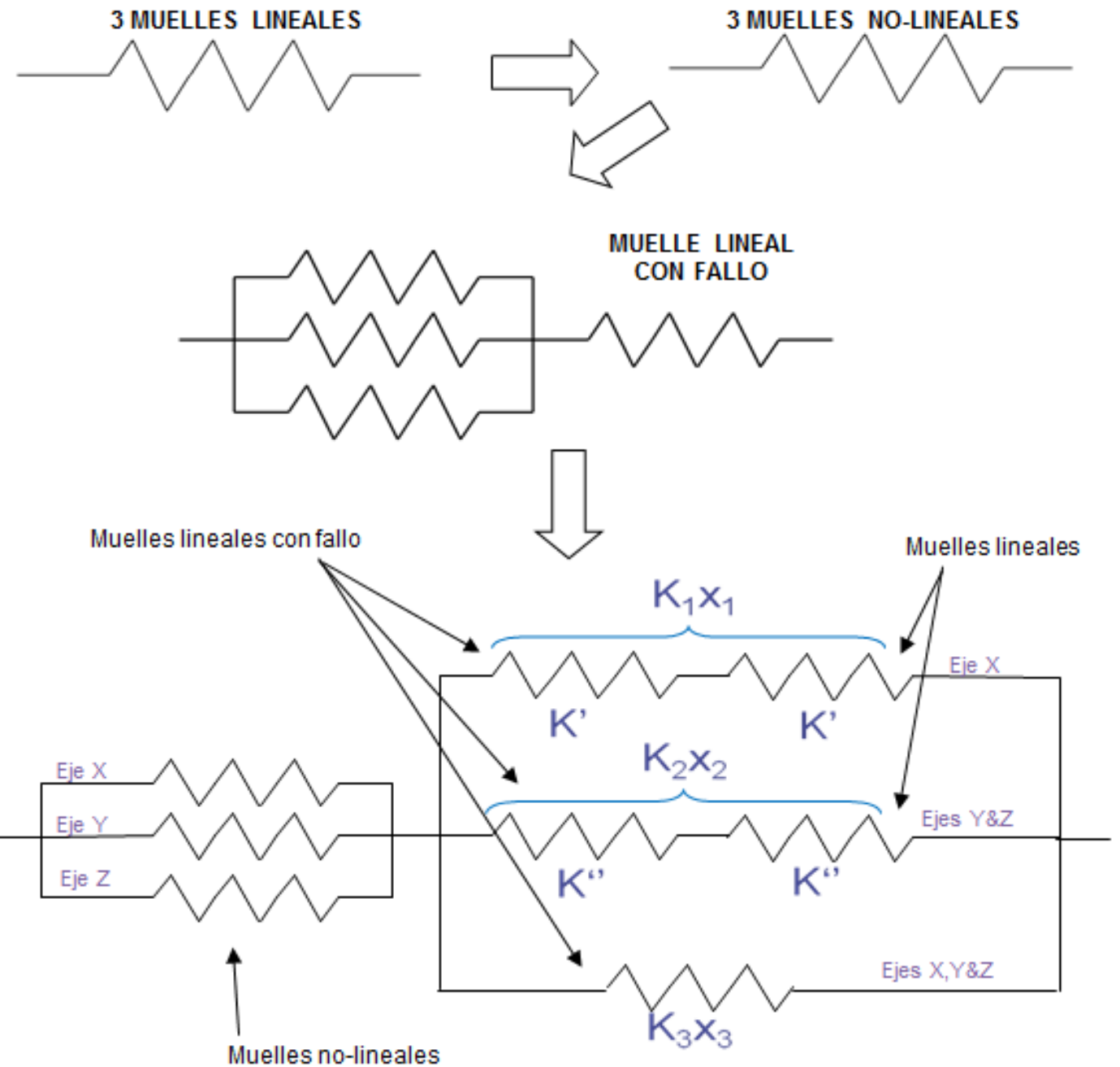

Figura 4.8: Evolución del modelo elastomérico y diagrama de muelles 
En los apoyos existen diferentes criterios de fallo según el eje, por lo tanto el diagrama propuesto no lo puede reproducir. Entonces, los muelles lineales con fallo se deben reemplazar por varios muelles lineales con fallo actuando en diferentes ejes. En resumen, los muelles lineales con fallo están en serie con 3 muelles no lineales traslacionales, como se puede ver en la figura 4.8. La filosofía del modelo es usar 3 muelles paralelos: uno de ellos trabaja en el eje X, otro en los ejes Y y Z y el otro en los ejes X, Y y Z. El objetivo del conjunto es obtener un criterio de fallo elipsoidal como el mostrado en la figura 4.9 donde el criterio de fallo para el eje $\mathrm{X}$ es diferente al de los ejes $\mathrm{Y}$ o Z y que sea compatible con los elementos disponibles en el software utilizado. Por lo tanto, mientras los valores de las fuerzas se encuentren dentro de las regiones demarcadas ningún muelle falla.

El criterio de fallo viene impuesto por $F_{x}$ y $F_{y}=F_{z}$. En los soft mounts o muelles delanteros la rigidez y la fuerza de la parte lineal sigue las siguientes ecuaciones:

$$
\left\{\begin{array} { l } 
{ K _ { x } = K _ { 1 } + K _ { 3 } } \\
{ K _ { y } = K _ { 2 } + K _ { 3 } } \\
{ K _ { z } = K _ { 2 } + K _ { 3 } }
\end{array} \quad \Rightarrow \quad \left\{\begin{array}{l}
F_{\mathrm{enX}}=\left(K_{1}+K_{3}\right) x_{x} \\
F_{\mathrm{enY}}=\left(K_{2}+K_{3}\right) x_{y} \\
F_{\mathrm{enZ}}=\left(K_{2}+K_{3}\right) x_{z}
\end{array}\right.\right.
$$

Por lo tanto, las fuerzas en los muelles 1,2 y 3 son:

$$
\left\{\begin{array}{l}
F_{\text {en1 }}=K_{1} x_{1}=K_{1} x_{x} \\
F_{\text {en2 }}=K_{2} x_{2}=K_{2} \sqrt{x_{y}^{2}+x_{z}^{2}} \\
F_{\text {en3 }}=K_{3} x_{3}=K_{3} \sqrt{x_{x}^{2}+x_{y}^{2}+x_{z}^{2}}
\end{array}\right.
$$

El criterio de fallo se basa en el desplazamiento máximo permitido para la zona lineal, $R$. Entonces, el criterio de fallo de los muelles 1,2 y 3 se puede escribir como $F_{1}$ fallo $=K_{1} R$, $F_{2}^{\text {fallo }}=K_{2} R$ and $F_{3}^{\text {fallo }}=K_{3} R$ respectivamente.

Durante la simulación, como $\sqrt{x_{x}^{2}+x_{y}^{2}+x_{z}^{2}} \geq x_{x}$ and $\sqrt{x_{x}^{2}+x_{y}^{2}+x_{z}^{2}} \geq \sqrt{x_{y}^{2}+x_{z}^{2}}$ el muelle 3 siempre rompe antes que el 1 y el 2 . Cuando el muelle 3 falla, las fuerzas en el muelle 1 y muelle 2 se incrementan y hacen que rompan a continuación.

$$
\begin{aligned}
& F_{\text {en1 }}=K_{1} x_{x} \stackrel{(3 \text { rompe })}{\Longrightarrow} F_{\text {en1 }}=K_{1} x_{x}+K_{3} x_{x} \stackrel{\text { normalmente }}{>} F_{1}^{\text {fallo }}
\end{aligned}
$$

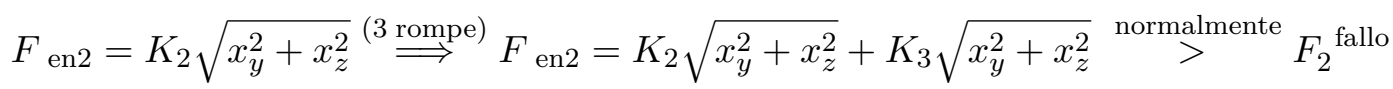

Por lo tanto, la secuencia de fallo es como sigue: el muelle 3 es siempre el primero en romper seguido por el muelle 2 ; finalmente rompe el muelle 1 ó, si la fuerza es en gran parte de componente $Y$ y $Z$, el muelle 1 se mantiene unido residualmente hasta que alguna carga pequeña en $X$ lo rompa (la rigidez del muelle 1 se elige con el criterio de ser lo menor posible).

Siguiendo estas ecuaciones se consigue reproducir la resistencia y rigidez del elastómero. 

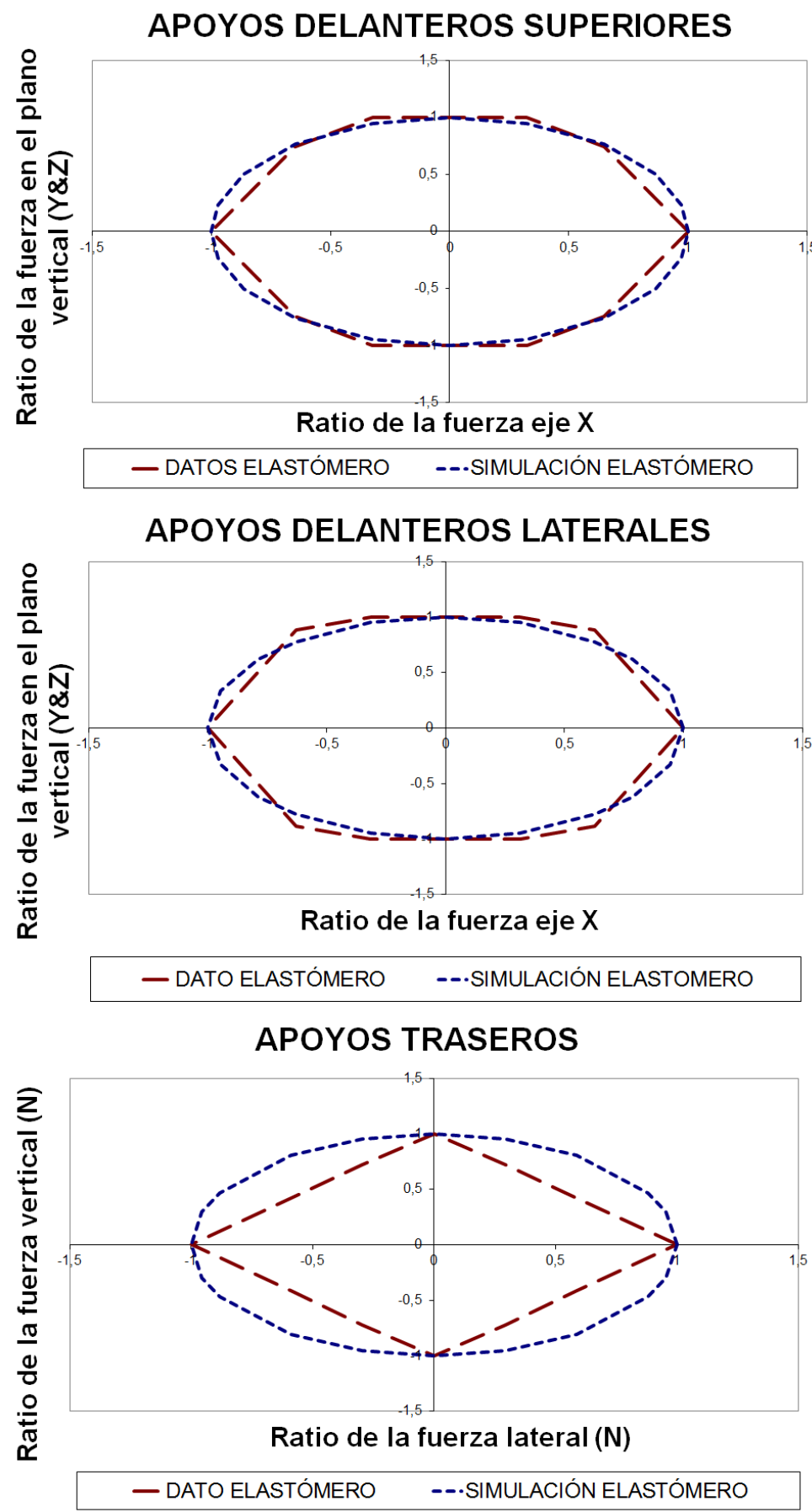

Figura 4.9: Área de fallo

\subsubsection{Consideraciones de los análisis explícitos}

Debido a que el modelo se analiza mediante MSC.Dytran, que es un solver explícito, el incremento de tiempo en cada paso del análisis es crucial. Tienen que ser suficientemente grande para poder analizar todo el fenómeno en un tiempo máquina (CPU) razonable pero asegurando 
la convergencia en cada paso de tiempo. La condición característica de estabilidad es la ecuación 2.3.1.2 (ver también MSC (2005)).

Para resolver esta inecuación con un paso de tiempo razonable, la malla del modelo FEM tiene que estar optimizada para que la longitud característica del elemento crítico sea lo mayor posible y la frecuencia de ese elemento sea lo menor posible.

El nivel de energía a lo largo de la pérdida de pala y, por lo tanto, a lo largo de la simulación es un excelente indicador del desarrollo del fenómeno. Las fuerzas aplicadas debidas a la pérdida de pala y el desplazamiento que producen realizan un trabajo e incrementan la energía del modelo. La mayoría de la energía se transforma en energía cinética del motor. Parte de la energía cinética, determinada por las propiedades de los elastómeros, alcanza la bancada y una cantidad aún menor puede incluso alcanzar el ala (en nuestro modelo se considera a través de las fuerzas que alcanzan las condiciones de contorno del ala). Adicionalmente, parte de la energía se convierte en energía interna y para ser más preciso energía elástica. La deformación del elemento absorbe esta energía elástica de manera reversible mientras se trabaje en el régimen lineal. Cuando uno de los elementos alcanza el nivel de esfuerzos plástico, el intercambio de energía se vuelve irreversible. Éste también es el caso cuando un elemento falla. Si esto ocurre, ese camino de carga se interrumpe. Estos sucesos hacen a cada caso de carga distinto, la simulación evoluciona y los casos se separan entre sí haciendo a cada uno único.

Además, por las características del solver explícito, es importante tener en consideración la energía 'hourglass' que son modos de deformación inherentes a los elementos placa de este tipo de solvers, ver MSC (2005). Son los llamados modos de energía nula o modos espurios de deformación. El elemento puede deformarse según esos modos sin producir ninguna fuerza. El nivel de energía de hourglass debe mantenerse entre ciertos niveles a lo largo de toda la simulación. Si no fuera así las velocidades nodales pueden diverger rápida y fácilmente hacia infinito, sobre todo cuando éstas sean paralelas al modo hourglass de deformación. Todos los tipos de energía considerados en el análisis están descritos en 4.4.4.1.

A continuación se muestran varios de los chequeos llevados a cabo en el análisis.

\subsubsection{Chequeos másicos $\mathrm{y}$ energéticos}

Se han tenido en cuenta las correspondientes propiedades másicas e inerciales para que el modelo reproduzca las propiedades másicas e inerciales de la estructura. Para llevar a cabo las simulaciones adecuadamente los resultados sólo se consideraron válidos cuando éstas llegaban hasta el final de la secuencia de roturas sin ninguna inestabilidad numérica y cumpliendo con los criterios energéticos. Además, como se ha mencionado es importante prestar atención a los parámetros energéticos, por ejemplo a los inherentes a los solvers explícitos (energía de hourglass). 
Toda la energía del modelo de cada caso de carga se estudia y se chequea. La energía que se introduce se compara con las energías del modelo (cinética, de distorsión, elástica o de los muelles, la perdida por las roturas,...).

Energía cinética de vigas/placas $\Longrightarrow E_{\text {kin beam } / \text { shell }}=\sum_{i} E_{\text {kin element i }}$

Energía cinética de masas $\Longrightarrow E_{\text {kin mass }}=\frac{1}{2} \sum_{i} \operatorname{mass}_{i} \times$ velocity $_{i}^{2}$

Energía interna $\Longrightarrow E_{\text {dis }}=\sum_{i} E_{\text {dis element i }}$

Energía de Hourglass $\Longrightarrow E_{\text {Hourglass }}=\sum_{i} E_{\text {Hourglass element i }}$

Energía de los muelles lineales $\Longrightarrow E_{\text {lin spring }}=\frac{1}{2} \sum_{i} \frac{\text { force }_{1}^{2}}{\text { stiffness }_{i}}$

Energía de los muelles no-lineales $\Longrightarrow E_{\text {non-lin spring }}=\int^{x}$ force $(s) d s$

$E_{\text {TOTAL }}=E_{\text {kin beam } / \text { shell }}+E_{\text {kin mass }}+E_{\text {dis }}+E_{\text {hourglass }}+E_{\text {lin spring }}+$ $+E$ nonlin spring

Y para terminar, la energía introducida es el trabajo que producen las fuerzas y los momentos aplicados:

$E_{\text {INTRODUCED }}=\int$ ext. force $d s+\int$ ext. torque $d \theta$

Por lo tanto, a lo largo de toda la simulación se compara por un lado la energía introducida por las fuerzas exteriores y se compara con la suma de todas las energías existentes en el modelo. En la figura 4.10 se cuantifican los valores de todas las energías que se han mencionado para un caso de carga. En este caso se puede ver como la energía introducida y la energía total se mantienen prácticamente iguales a lo largo de todo el proceso.

\subsubsection{Chequeo de modos propios}

Como en la mayoría de los procedimientos de chequeos de modelos de elementos finitos se debe realizar un chequeo de modos propios. Sin embargo, los softwares comerciales con solvers explícitos no tienen un análisis de modos como tal, sobre todo debido a que la solución es resuelta en el dominio del tiempo.

En este caso, se ha desarrollado un procedimiento para evaluar la vibración libre de la estructura. Se deforma la estructura según el posible modo y se deja vibrar libremente, entonces si es un modo propio se moverá permanentemente siguiendo el modo y armónicamente a la frecuencia del modo. Si la deformación no correspondiera a un modo puro, la evolución no es un armónico puro, se movería según los modos propios que hayan sido excitados y a sus respectivas frecuencias. Si no hay amortiguamiento definido la respuesta transitoria debida a las condiciones iniciales no se amortigua rápidamente y la respuesta no es siempre un modo puro. En el modelo los modos propios se simulan correctamente como se muestra en la figura 4.11, que es una captura de la evolución habiendo excitado un modo propio. 


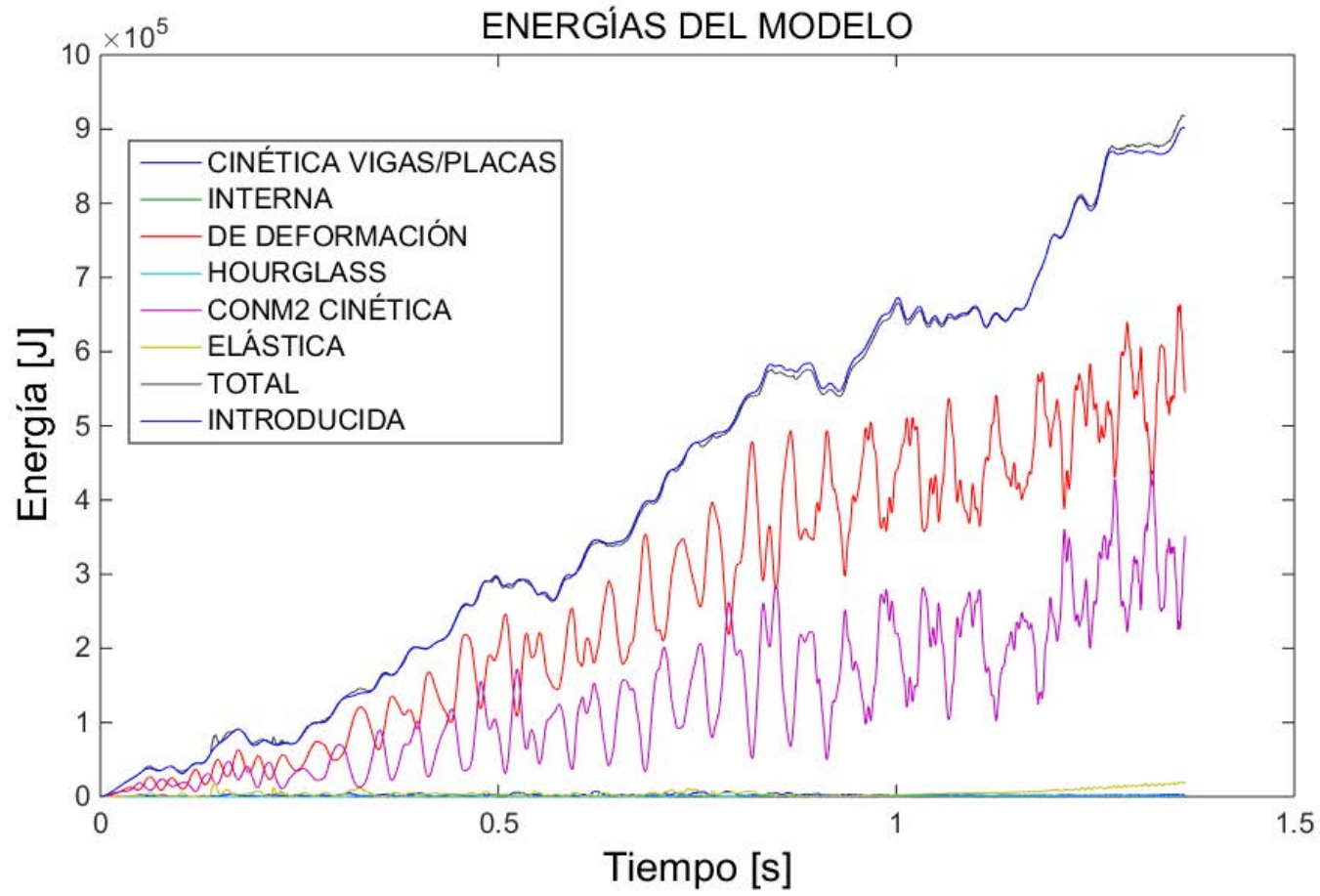

Figura 4.10: Energías del modelo

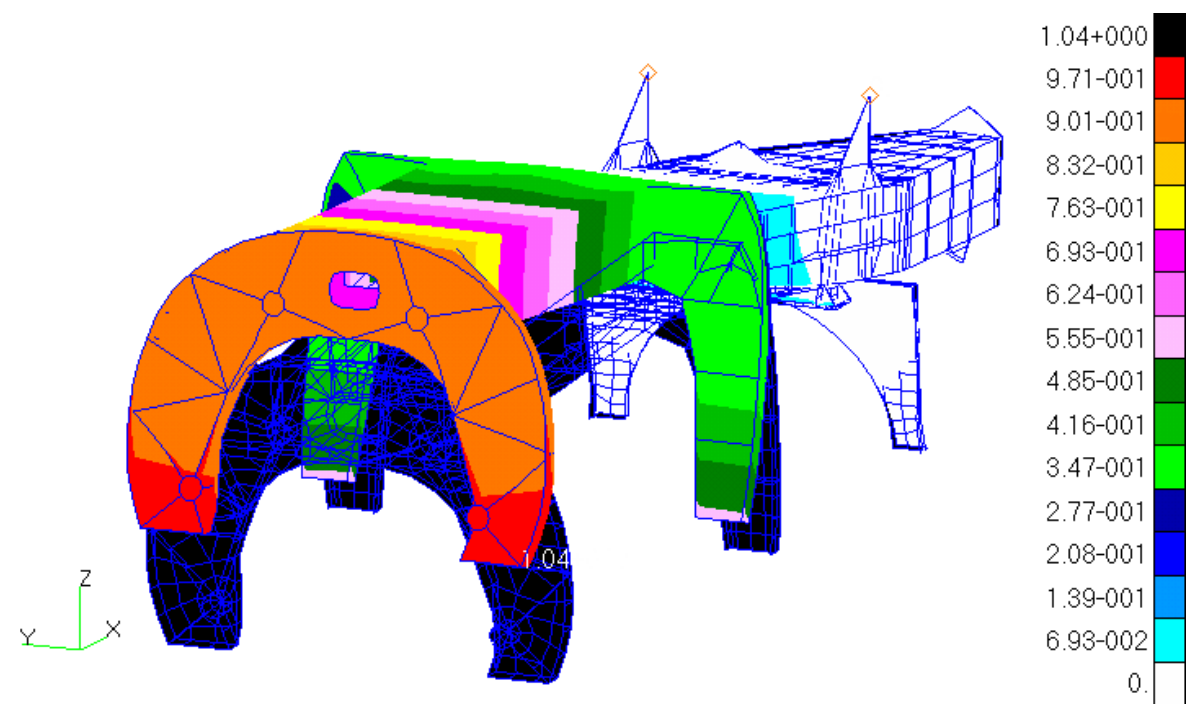

Figura 4.11: Captura de la evolución habiendo excitado un modo propio

\subsubsection{Casos de carga analizados}

La seguridad de la aeronave depende de la selección de las cargas de diseño y de los requisitos que a la postre también define la rigidez estructural y las propiedades másicas. Se analizan varios casos de carga, algunos de ellos son acerca de las condiciones iniciales desde las que se produce el fenómeno de la pérdida de pala, no es lo mismo encontrarse en pleno aterrizaje que en vuelo de crucero a gran altura que realizando una maniobra, tampoco es igual si se rompe 
toda la pala o solo una parte, y otra son distintas variaciones de parámetros involucrados en el propio fenómeno, como son las rigideces de las uniones. Los parámetros concretos analizados son:

- Posición angular: La posición angular de la pala en el plano de la hélice cuando se produce la pérdida puede influir en el suceso.

- Puntos de la envuelta de vuelo: La pérdida de pala se analiza mayormente como un fenómeno aislado pero se produce partiendo de una condición de vuelo determinada. También se analiza el efecto aislado de la gravedad

- Sensibilidad a las rpm: La velocidad de rotación de la hélice cambia con la condición de vuelo por lo que el fenómeno puede producirse con distintas velocidades.

- Sensibilidad a la rigidez de la unión bancada-ala: Como se ha mencionado anteriormente la rigidez de la unión se varía.

- Momento y forma de introducción de las fuerzas: La forma de introducción de las cargas puede variarse. Normalmente las cargas se aplican instantáneamente pero en este caso se analiza suavizando esta introducción ya que dependiendo del tipo de rotura que se produzca las cargas actuarán súbitamente o progresivamente.

- Tamaño de la pala perdida: La pala puede desprenderse totalmente o sólo una parte.

- Flexibilidad del ala: El objetivo es analizar la influencia de un ala flexible (el $1^{e r}$ modo) en la simulación. La variación de estos casos de carga consiste en introducir la rigidez y masa del ala equivalente a su primer modo de flexión.

- Amortiguamiento estructural: Su variación puede tener influencia en la respuesta de la estructura.

En los apartados siguientes se estudia cómo introducir las variaciones de los diferentes parámetros individualmente.

\subsubsection{Posición angular}

Las fuerzas y momentos aplicados en el motor siguen las siguientes ecuaciones: $F_{y}=$ $-F_{c} \cos (\omega t+\theta), F_{z}=-F_{c} \sin (\omega t+\theta), M_{y}=M_{\text {oop }} \sin (\omega t+\theta)$ y $M_{z}=-M_{\text {oop }} \cos (\omega t+\theta)$ donde $\theta$ indica la posición angular dentro del plano de la hélice de la pala perdida. Se estudiaron distintos valores de $\theta, 0^{\circ}$ (caso nominal), $45^{\circ}, 90^{\circ}, 135^{\circ}, 180^{\circ}, 225^{\circ}, 270^{\circ}$ y $315^{\circ}$. Los distintos casos se muestran en la figura 4.12 . 


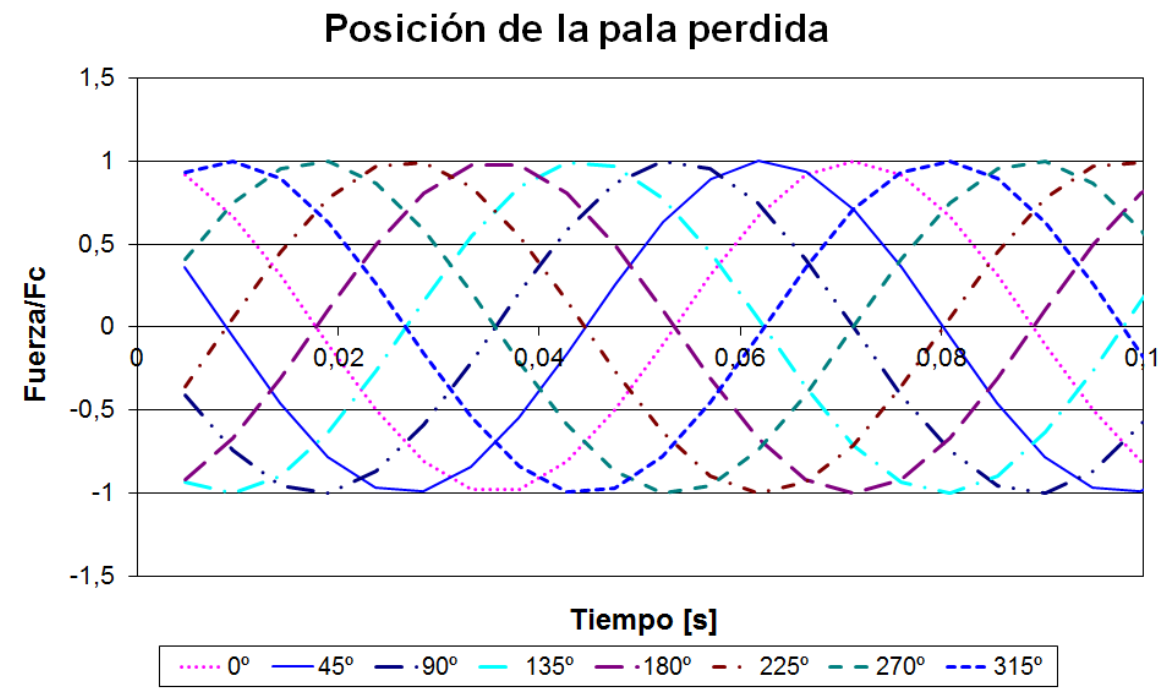

Figura 4.12: Influencia de los distintos ángulos de la pala de la hélice perdida en las fuerzas

\subsubsection{Puntos de la envolvente de vuelo}

En este grupo de casos de carga se parte de distintos puntos de la envolvente de vuelo y para poder introducir estas condiciones iniciales se realizan unos análisis previos. Si por ejemplo el punto de la envolvente tiene un factor de carga 1 no es lo mismo aplicarlo como condición inicial a la vez que la pérdida de pala que aplicarlo dejar que el modelo se estabilice y entonces aplicar la pérdida de pala, un ejemplo de estabilización se puede ver en la figura 4.13 en donde se puede ver que al aplicar las condiciones del punto de la envolvente de vuelo el valor de la señal parte de 1 en vez de 0 cuando se aplique la pérdida de pala. En este análisis previo se aplican las cargas de un punto de la envolvente de vuelo (la gravedad, empuje, par motor,... ), y a continuación se aplica la pérdida de pala. Estos puntos de la envolvente de vuelo son: velocidad $V_{b}$ a nivel del mar $(655 \mathrm{rpm})$, velocidad $V_{c}$ a nivel del mar $(842 \mathrm{rpm})$, velocidad $V_{c}$ a una altitud determinada (690 rpm) y aplicando sólo y aisladamente la gravedad (Grav).

\subsubsection{Sensibilidad a las rpm}

En estos casos de carga se analiza la variación de la frecuencia de la carga que se corresponde con la velocidad de rotación de la hélice (ver figura 4.14). Por lo tanto, si la hélice gira a menor velocidad se producirá unas fuerzas de desequilibrio menores si se rompe la pala llegando al extremo de que no se produce desequilibrio si la pala se rompe estando parada. Si la variación se introduce considerando $\alpha=\frac{\omega}{\omega_{n o m}}$ las ecuaciones de los módulos de la fuerza y el momento quedarían:

$$
\beta=\frac{F_{c}(\omega)}{F_{c}}=\alpha
$$




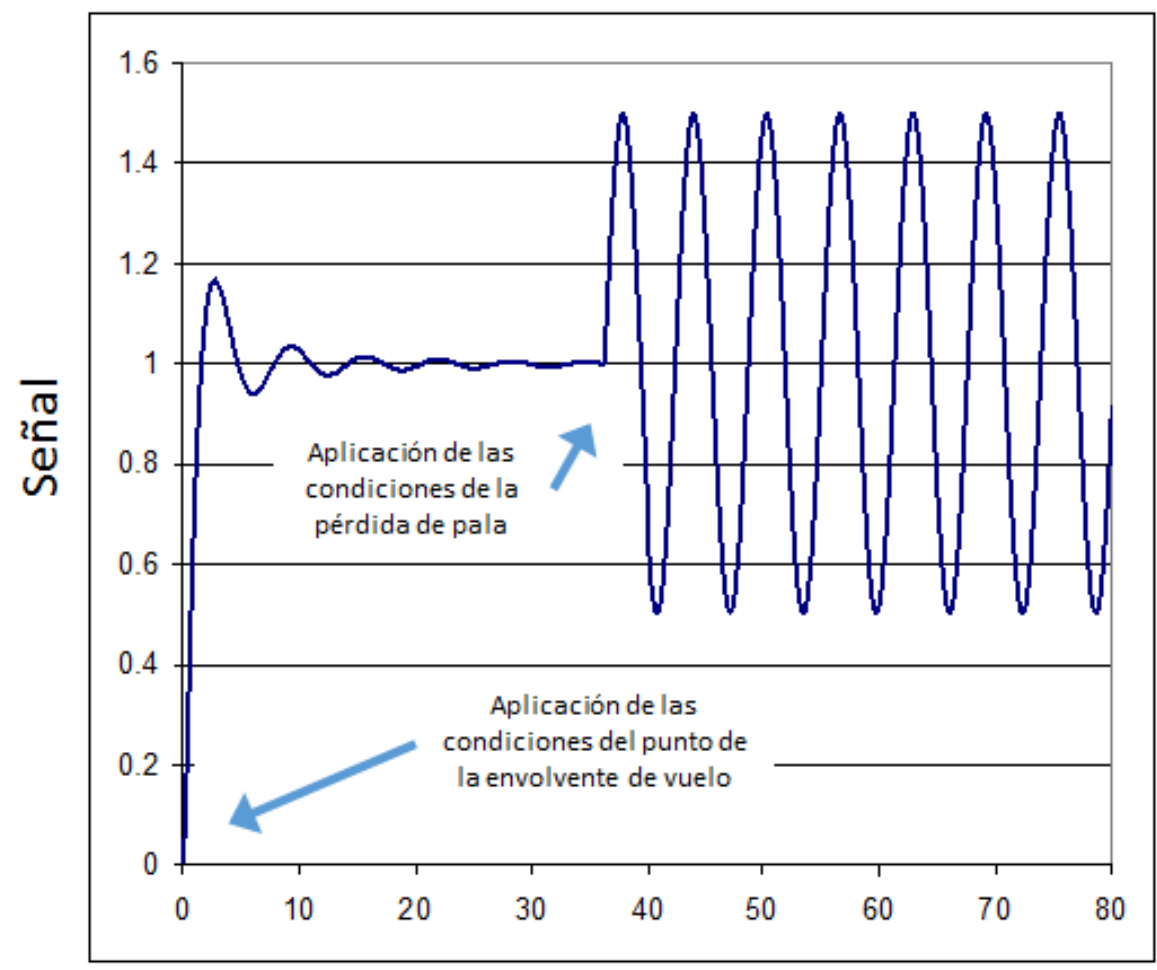

Tiempo $[s]$

Figura 4.13: Ejemplo de la estabilización antes de aplicar la carga

$$
\gamma=\frac{M_{o o p}(\omega)}{M_{o o p}}=\alpha
$$

Lo que muestra que las fuerzas y momentos decrecen cuando la frecuencia decrece. Los casos de carga son a $842 \mathrm{rpm}, 780 \mathrm{rpm}, 720 \mathrm{rpm}$ y $660 \mathrm{rpm}$.

\subsubsection{Sensibilidad a la rigidez de la unión bancada-ala}

Como ya se ha mencionado se varía su módulo de rigidez desde su valor nominal, ratio 1 , a un valor mucho menos rígido, ratio 0.01 . Los valores escogidos son: 1, 0.75, 0.6, 0.5, 0.33, 0.25, $0.2,0.1,0.05,0.02$ y 0.01 .

\subsubsection{Momento y forma de introducción de las fuerzas}

La forma de introducción de las cargas puede variarse. Normalmente las cargas se aplican instantáneamente pero en este caso se analiza suavizando esta introducción ya que dependiendo del tipo de rotura que se produzca las cargas actuarán súbitamente o progresivamente. En estos casos de carga se analiza la forma en que se introduce la carga. En el caso nominal la carga se aplica instantáneamente, sin embargo, esta introducción puede ser más suave (ver la figura 4.15). Dependiendo de cómo sea la rotura las cargas actuarán de una manera o de otra, aquí se 


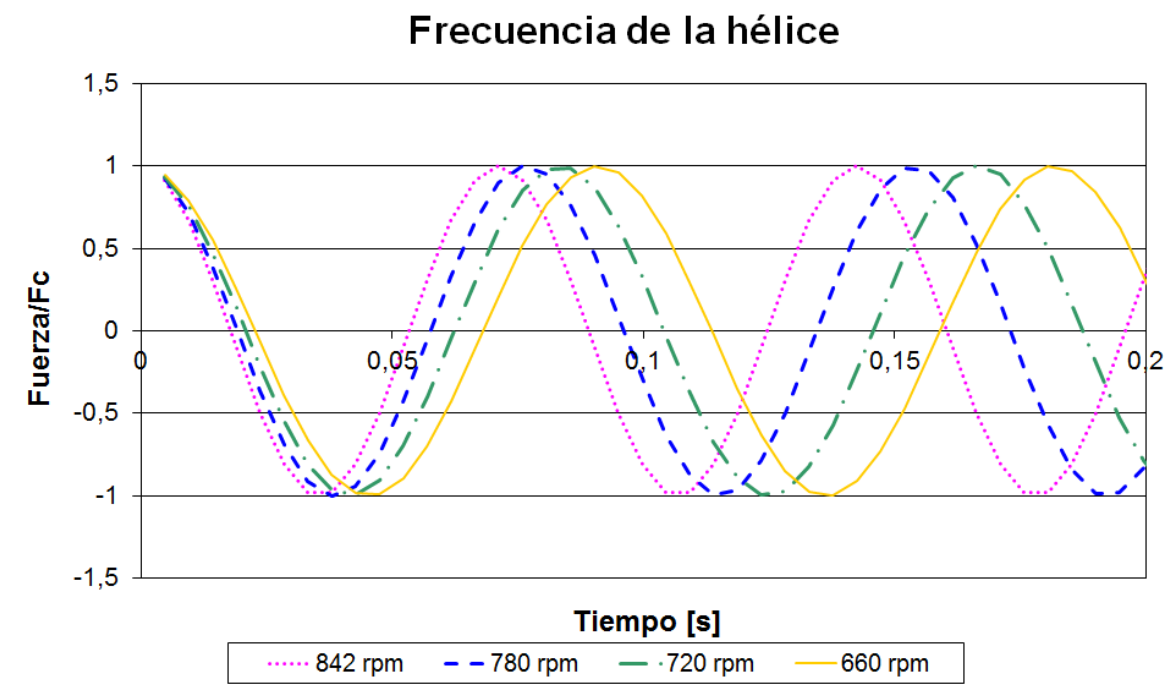

Figura 4.14: Variación de la frecuencia rpm en las fuerzas en función del tiempo

estudian dos maneras principales de introducir la carga, de forma lineal y cosenoidal. Además, la entrada de la carga se va a introducir cuando la hélice haya recorrido $15^{\circ}, 30^{\circ} \mathrm{y} 45^{\circ}$.

\section{Introducción de la fuerza}

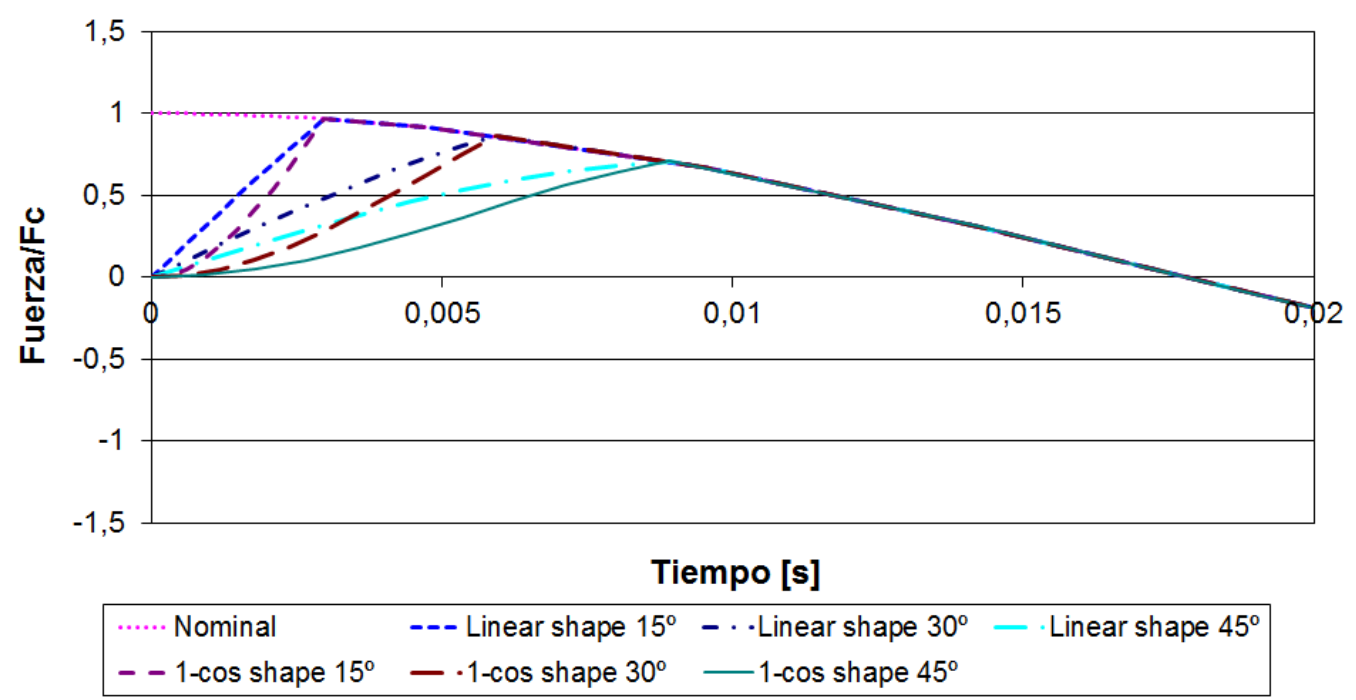

Figura 4.15: Influencia de la forma y duración de la introducción de la carga en las fuerzas

\subsubsection{Tamaño de la pala perdida}

En estos casos de carga se estudia la influencia del tamaño de pala perdida en los resultados. Para ello se introduce el parámetro que muestra el ratio entre el radio de pala desprendida frente 
al radio total de las palas, $\alpha=\frac{r}{R}$. Se han considerado diferentes casos, desde un ratio de 1 hasta 0 . Son los siguientes:

$$
\alpha=\frac{r}{R}=0,0.01,0.2,0.4,0.6 \text { у } 0.8
$$

Esté término se introduce en el modelo de la siguiente manera:

$$
\begin{gathered}
\beta=\frac{F_{c}(r)}{F_{c}}=1-\alpha^{2} \\
\gamma=\frac{M_{o o p}(r)}{M_{o o p}}=1-\alpha
\end{gathered}
$$

Por lo tanto, las fuerzas y momentos disminuyen cuando el ratio aumenta.

\subsubsection{Amortiguamiento estructural}

En estas simulaciones se estudian los efectos del amortiguamiento estructural. La variación de estos casos de carga consiste en introducir cierto \% de amortiguamiento estructural en el modelo. Las ecuaciones que rigen este fenómeno son:

$$
M a^{n}+C v^{n}+F_{i n t}^{n}=F_{e x t}^{n} \Longrightarrow C=\frac{2 \beta}{\Delta t} M
$$

y como

$$
\beta=\eta \omega \Delta t
$$

donde $\beta$ es el parámetro dinámico de relajación definido en Dytran, $\eta$ es el amortiguamiento estructural, $\Delta t$ es el paso de tiempo y $\omega$ es la frecuencia del sistema. Finalmente se considera amortiguar el sistema entre un $0 \%$ y un $5 \%$ la primera frecuencia natural del sistema. Los casos de carga se denominan: 0.01, 0.02, 0.03, 0.04 y 0.05 .

\subsubsection{Flexibilidad del ala}

En estos casos de carga se analiza el efecto de considerar un ala flexible en vez de totalmente rígida. En el caso nominal se considera el ala totalmente rígida. Las ecuaciones que rigen el comportamiento de la flexibilidad del ala son:

$$
\omega_{\text {wing }}=\sqrt{\frac{k_{w i n g}}{m_{w i n g}}}
$$

Por lo tanto se introduce una masa y un muelle y se asume que las propiedades están uniformemente distribuidas. En cada condición de contorno se tiene en cuenta este comportamiento mediante la variación de su correspondiente masa y rigidez. Los valores finalmente escogidos son: $1.57 \mathrm{~Hz}, 1.65 \mathrm{~Hz}, 1.68 \mathrm{~Hz}$ y $1.76 \mathrm{~Hz}$. 


\subsubsection{Resultados de los casos de carga}

Los análisis se realizan para analizar la influencia de varios parámetros mediante distintos resultados como:

- Fuerzas en los muelles (fuerza vs. tiempo y rotura). En este caso se registra la distinta fuerza en todos los muelles del sistema hasta que falla, momento en el que deja de soportar carga.

- Secuencia de rotura de los elastómeros. Estos resultados se pueden ver en la figura 4.16 donde en cada caso de carga se muestra con el color y la longitud de la columna el orden de rotura. De la figura se puede obtener la secuencia de fallo ya que para cada caso hay una columna con el valor $1,2,3,4,5$ y 6 indicando la secuencia.

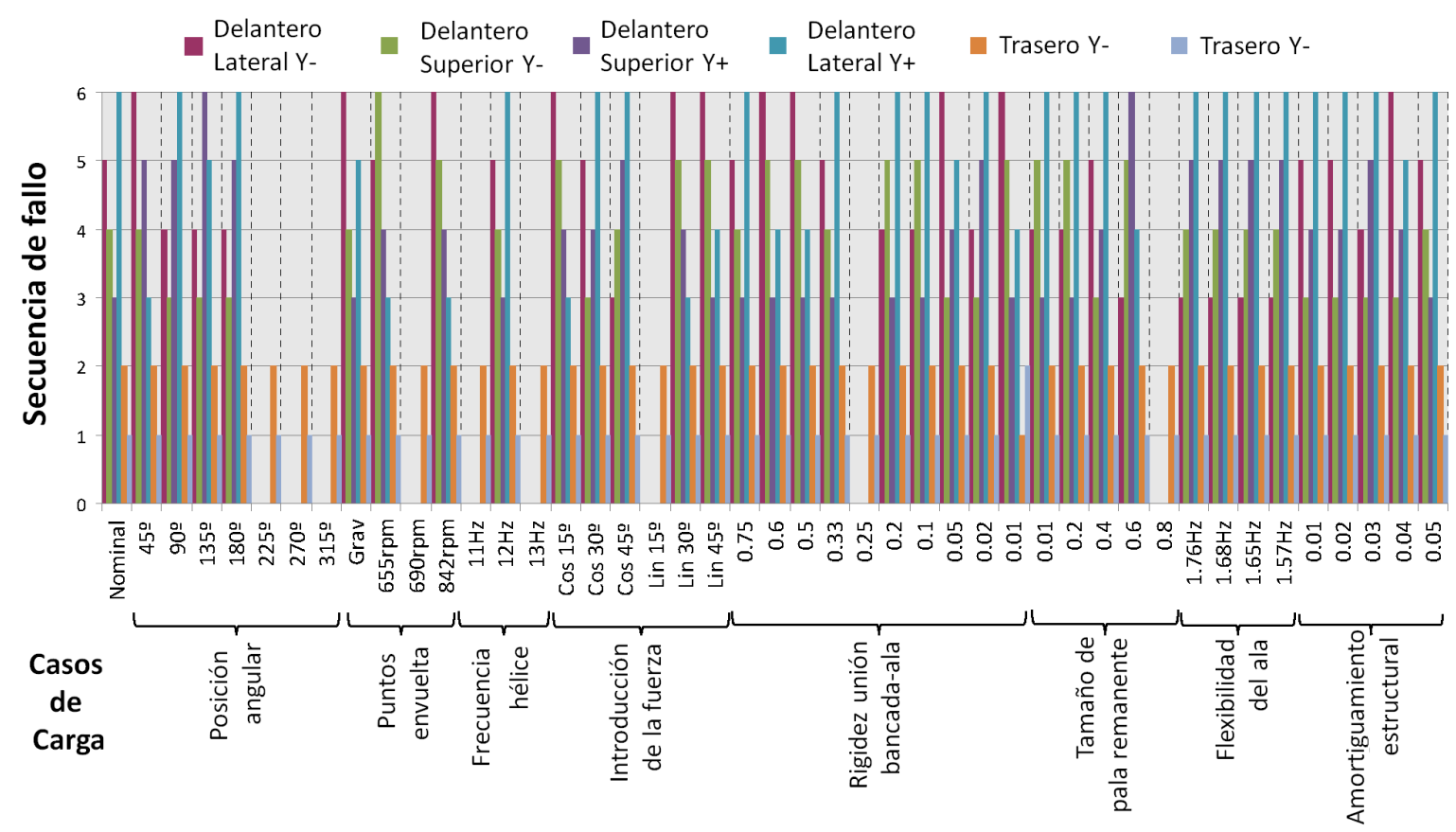

Figura 4.16: Secuencia de fallos de los apoyos para un caso de carga (1 para el primer fallo,..., 6 para el último fallo)

- Fuerzas en las condiciones de contorno. Estos resultados se pueden ver en la figura 4.17, esta figura muestra la fuerza en $\mathrm{Z}$ de uno de los apoyos, existen tantas gráficas como direcciones y apoyos, es decir, como se mostraba en la figura 4.6 hay en total 6 gráficas por las direcciones de los puntos con condiciones de contorno (puntos 1-4).

- Elementos plastificados. Estos resultados para un caso de carga se traduce en la figura 4.18 en donde se observan los elementos que han plastificado en algún momento a lo largo de la simulación. 


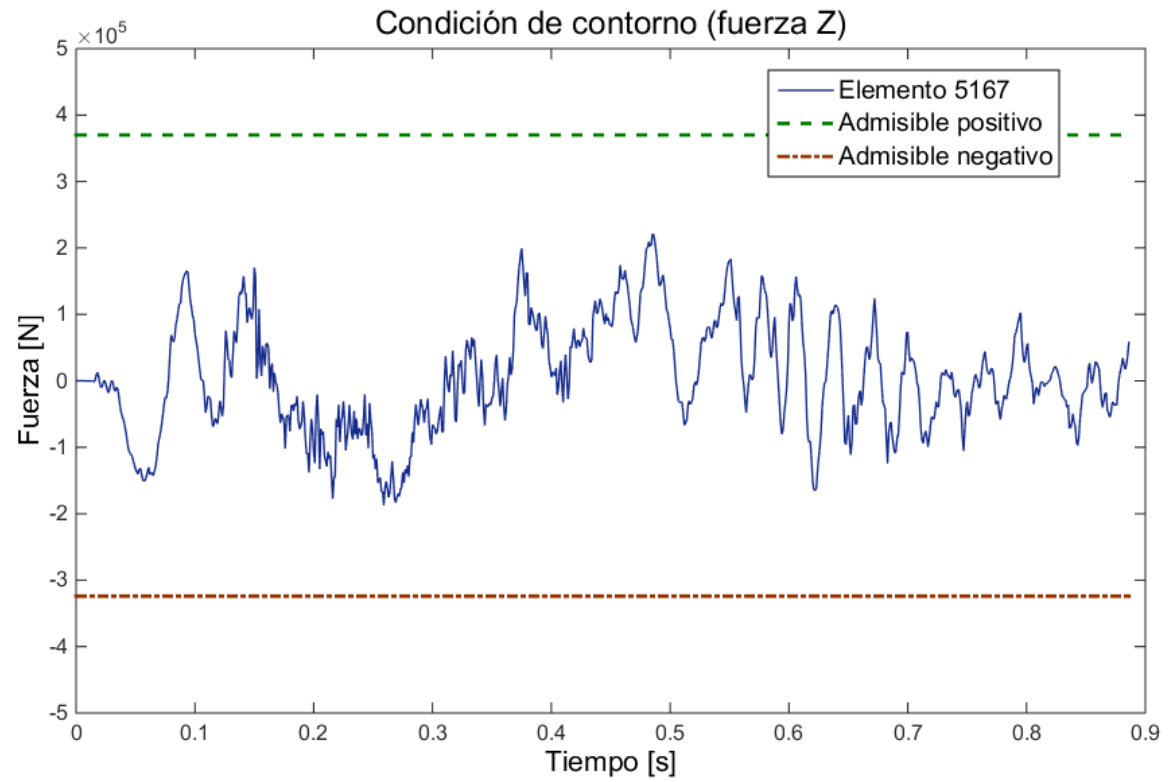

Figura 4.17: Fuerzas en las condiciones de contorno

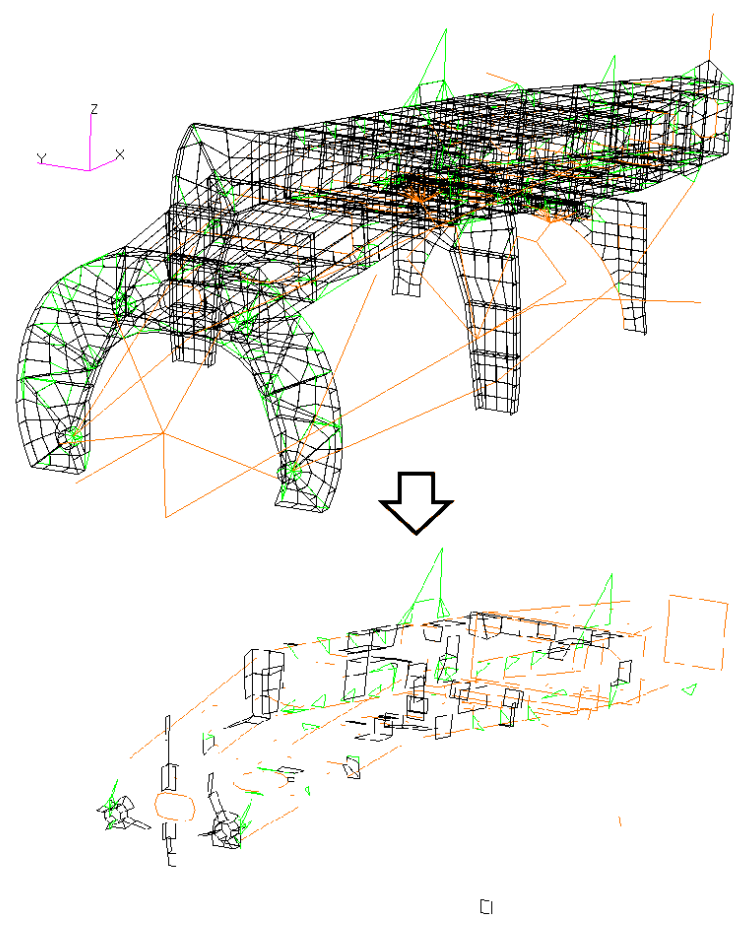

Figura 4.18: Elementos FEM (arriba) y elementos plastificados de un caso de carga (abajo)

- Energías del modelo. En este caso y como ya se ha explicado en la sección 4.4.4.1 los resultados son del tipo de la figura 4.10 en donde se pueden ver distintos tipos de energías y en donde se ha chequeado en todo momento que la energía total del sistema sea igual a la energía introducida. 


\subsubsection{Resultados obtenidos}

En este apartado se muestra un resumen de todos los resultados obtenidos. Como se ha comentado, el paso de tiempo es crucial para no penalizar el tiempo de cálculo y por eso se ha optimizado el modelo para que el mínimo paso de tiempo aplicado haya sido $1,8 e^{-6}$ y siendo en la mayoría de ellos $1,8 e^{-5}$, con lo que se ha conseguido simular varios ciclos de hélice. Se muestran en la tabla 4.1 todos los casos de carga analizados agrupándolos por grupos de casos de carga con el tiempo y los ciclos analizados así como el incremento de tiempo utilizado. Los tiempos de simulación suelen ser más largos en los casos de carga en los que la rotura no se ha producido o se produce muy tarde, se ha llegado en algún caso a 2 segundos de simulación. Esta información se encuentra también en la figura 4.22 en donde se muestra el número de ciclos simulados de los distintos casos de carga y, más adelante, en la figura 4.19 donde se muestra los tiempos de simulación que se reprodujeron.

Tiempo de Simulación

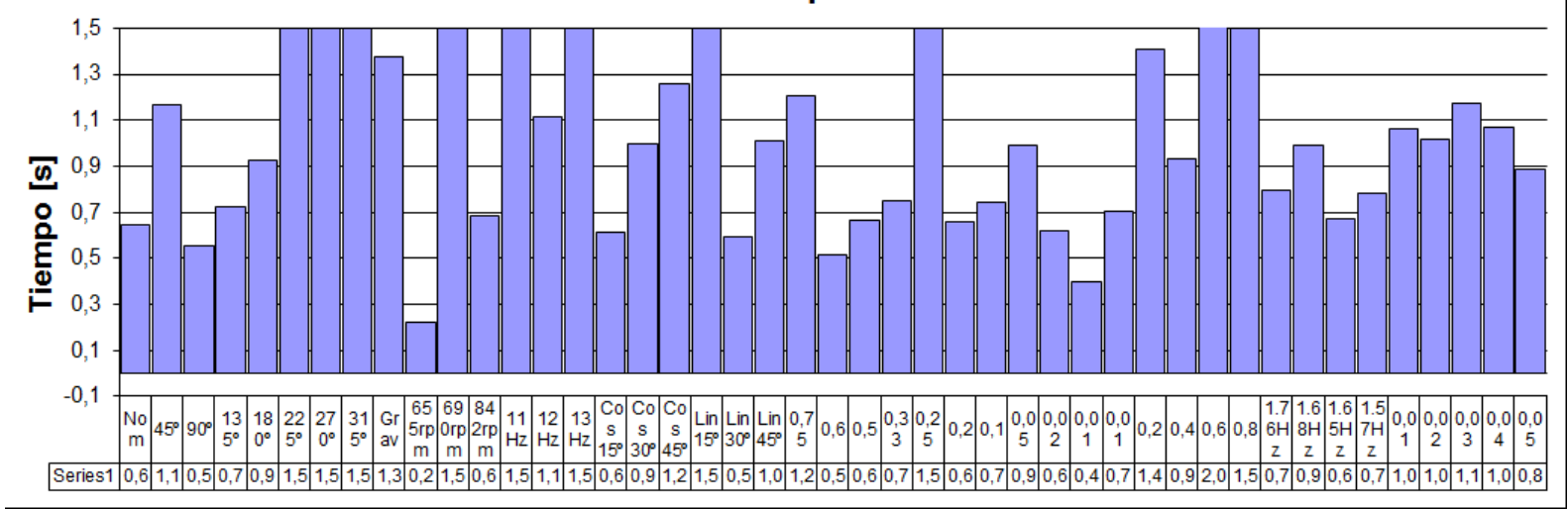

Figura 4.19: Tiempo de la simulación en los distintos casos de carga

La correcta simulación se consiguió para la mayoría de los casos de carga (más del $80 \%$ ). En la tabla 4.2 se muestran los intervalos de tiempo en los que se produjeron los fallos de los distintos apoyos. El intervalo que se muestra es desde que el primer muelle representativo del elastómero falla hasta que lo hace el último liberando el movimiento. Como ya se ha comentado cuando se produce la rotura de uno de los muelles del elastómero es ahí cuando prácticamente deja de poder transmitir fuerzas y, enseguida, si sigue solicitado romperán todos. Cuando el intervalo es más largo es porque aunque está unido no ha tenido que transmitir nada de fuerza, fuerza que lo haría romper. Como se puede observar prácticamente en la mayoría de los casos de carga el motor queda liberado en menos de 1 segundo.

Además, se ha identificado la secuencia de rotura para la mayoría de los casos de carga. Las secuencias se pueden ver en la figura 4.23. Un resumen de las secuencias de fallo más probables se puede ver en la tabla 4.3 en la que se muestra el número de veces que rompe el apoyo en $1^{e r}, 2^{o}, 3^{o}, 4^{o}, 5^{o}$ y $6^{o}$ lugar. Por lo tanto, la secuencia de rotura más probable es que primero 


\begin{tabular}{|c|c|c|c|c|c|}
\hline Caso de Carga & CASO & $\mathrm{Hz}$ & Ciclos & Tiempo(s) & $\Delta t\left(10^{-5} s\right)$ \\
\hline NOMINAL & Nom & 14,03 & 9,02 & 0,643 & 0,18 \\
\hline \multirow{7}{*}{$\begin{array}{l}\text { Posición } \\
\text { angular }\end{array}$} & $45^{\circ}$ & 14,03 & 16,38 & 1,168 & 0,18 \\
\hline & $90^{\circ}$ & 14,03 & 7,75 & 0,553 & 0,09 \\
\hline & $135^{\circ}$ & 14,03 & 10,16 & 0,724 & 0,18 \\
\hline & $180^{\circ}$ & 14,03 & 13,03 & 0,929 & 0,18 \\
\hline & $225^{\circ}$ & 14,03 & 21,05 & 1,500 & 0,04 \\
\hline & $270^{\circ}$ & 14,03 & 21,05 & 1,500 & 0,06 \\
\hline & $315^{\circ}$ & 14,03 & 21,05 & 1,500 & 0,06 \\
\hline \multirow{4}{*}{$\begin{array}{c}\text { Puntos } \\
\text { de la } \\
\text { envolvente } \\
\text { de vuelo }\end{array}$} & Grav & 14,03 & 19,32 & 1,377 & 0,06 \\
\hline & $655 \mathrm{rpm}$ & 10,91 & 2,42 & 0,222 & 0,18 \\
\hline & $690 \mathrm{rpm}$ & 11,50 & 17,25 & 1,500 & 0,03 \\
\hline & $842 r p m$ & 14,03 & 9,61 & 0,685 & 0,18 \\
\hline \multirow{3}{*}{$\begin{array}{l}\text { Sensibilidad } \\
\text { a las } \\
\text { rpm }\end{array}$} & $11 \mathrm{~Hz}$ & 11,00 & 16,50 & 1,500 & 0,09 \\
\hline & $12 \mathrm{~Hz}$ & 12,00 & 13,38 & 1,115 & 0,04 \\
\hline & $13 \mathrm{~Hz}$ & 13,00 & 19,50 & 1,500 & 0,04 \\
\hline \multirow{6}{*}{$\begin{array}{l}\text { Momento y } \\
\text { forma de } \\
\text { entrada de } \\
\text { la carga }\end{array}$} & $\operatorname{Cos} 15^{\circ}$ & 14,03 & 8,61 & 0,614 & 0,18 \\
\hline & $\operatorname{Cos} 30^{\circ}$ & 14,03 & 14,00 & 0,998 & 0,09 \\
\hline & $\operatorname{Cos} 45^{\circ}$ & 14,03 & 17,68 & 1,260 & 0,04 \\
\hline & $\operatorname{Lin} 15^{\circ}$ & 14,03 & 21,05 & 1,500 & 0,09 \\
\hline & $\operatorname{Lin} 30^{\circ}$ & 14,03 & 8,33 & 0,594 & 0,18 \\
\hline & $\operatorname{Lin} 45^{\circ}$ & 14,03 & 14,17 & 1,010 & 0,06 \\
\hline \multirow{10}{*}{$\begin{array}{l}\text { Sensibilidad } \\
\text { a la } \\
\text { rigidez } \\
\text { de la unión } \\
\text { bancada } \\
\text { ala }\end{array}$} & 0,75 & 14,03 & 16,92 & 1,206 & 0,09 \\
\hline & 0,6 & 14,03 & 7,27 & 0,518 & 0,18 \\
\hline & 0,5 & 14,03 & 9,34 & 0,666 & 0,18 \\
\hline & 0,33 & 14,03 & 10,52 & 0,750 & 0,18 \\
\hline & 0,25 & 14,03 & 21,05 & 1,500 & 0,03 \\
\hline & 0,2 & 14,03 & 9,21 & 0,656 & 0,18 \\
\hline & 0,1 & 14,03 & 10,38 & 0,740 & 0,18 \\
\hline & 0,05 & 14,03 & 13,94 & 0,993 & 0,09 \\
\hline & 0,02 & 14,03 & 8,71 & 0,621 & 0,18 \\
\hline & 0,01 & 14,03 & 5,61 & 0,400 & 0,18 \\
\hline \multirow{5}{*}{$\begin{array}{l}\text { Ratio } \\
\text { de pala } \\
\text { remanente }\end{array}$} & 0,01 & 14,03 & 9,92 & 0,707 & 0,18 \\
\hline & 0,2 & 14,03 & 21,05 & 1,405 & 0,04 \\
\hline & 0,4 & 14,03 & 13,04 & 0,929 & 0,18 \\
\hline & 0,6 & 14,03 & 28,80 & 2,053 & 0,18 \\
\hline & 0,8 & 14,03 & 21,05 & 1,500 & 0,18 \\
\hline \multirow{4}{*}{$\begin{array}{c}\text { Flexibilidad } \\
\text { del ala }\end{array}$} & $1,76 \mathrm{~Hz}$ & 14,03 & 11,17 & 0,796 & 0,18 \\
\hline & $1,68 \mathrm{~Hz}$ & 14,03 & 13,93 & 0,993 & 0,18 \\
\hline & $1,65 \mathrm{~Hz}$ & 14,03 & 9,42 & 0,671 & 0,09 \\
\hline & $1,57 \mathrm{~Hz}$ & 14,03 & 10,98 & 0,782 & 0,18 \\
\hline \multirow{5}{*}{$\begin{array}{c}\text { Factor de } \\
\text { amortiguamiento } \\
\text { estructural }\end{array}$} & 0,01 & 14,03 & 14,89 & 1,062 & 0,18 \\
\hline & 0,02 & 14,03 & 14,25 & 1,016 & 0,18 \\
\hline & 0,03 & 14,03 & 16,47 & 1,174 & 0,18 \\
\hline & 0,04 & 14,03 & 14,96 & 1,066 & 0,18 \\
\hline & 0,05 & 14,03 & 12,42 & 0,885 & 0,18 \\
\hline
\end{tabular}

Tabla 4.1: Ciclos, tiempo analizado y paso de tiempo de mínimo en los casos de carga 


\begin{tabular}{|c|c|c|c|c|c|c|c|}
\hline CASO DE CARGA & CASO & $\begin{array}{c}\text { Fallo apoyo } \\
\text { Lateral Y+ (s) }\end{array}$ & $\begin{array}{c}\text { Fallo apoyo } \\
\text { Lateral Y- (s) }\end{array}$ & $\begin{array}{c}\text { Fallo apoyo } \\
\text { Superior Y+ (s) }\end{array}$ & $\begin{array}{c}\text { Fallo apoyo } \\
\text { Superior Y- (s) }\end{array}$ & $\begin{array}{c}\text { Fallo apoyo } \\
\text { Trasero Y+ (s) }\end{array}$ & $\begin{array}{c}\text { Fallo apoyo } \\
\text { Trasero Y- (s) }\end{array}$ \\
\hline NOMINAL & Nom & $0,605-0,643$ & $0,552-0,552$ & $0,401-0,402$ & $0,621-0,623$ & $0,142-0,142$ & $0,139-0,182$ \\
\hline \multirow{7}{*}{$\begin{array}{l}\text { Posición } \\
\text { angular }\end{array}$} & $45^{\circ}$ & $1,150-1,168$ & $1,098-1,107$ & $1,101-1,122$ & $0,959-1,066$ & $0,132-0,179$ & $0,083-0,083$ \\
\hline & $90^{\circ}$ & $0,501-0,501$ & $0,499-0,500$ & $0,503-0,509$ & $0,552-0,553$ & $0,079-0,080$ & $0,075-0,076$ \\
\hline & $135^{\circ}$ & $0,689-0,697$ & $0,650-0,671$ & $0,700-0,701$ & $0,692-0,724$ & $0,161-0,184$ & $0,071-0,071$ \\
\hline & $180^{\circ}$ & $0,838-0,839$ & $0,731-0,731$ & $0,844-0,845$ & $0,871-0,929$ & $0,177-0,217$ & $0,132-0,132$ \\
\hline & $225^{\circ}$ & - & - & - & - & $0,086-0,086$ & $0,078-0,078$ \\
\hline & $270^{\circ}$ & - & - & - & - & $0,076-0,077$ & $0,069-0,069$ \\
\hline & $315^{\circ}$ & - & - & - & - & $0,071-0,071$ & $0,063-0,063$ \\
\hline \multirow{4}{*}{$\begin{array}{c}\text { Puntos } \\
\text { de la } \\
\text { envolvente } \\
\text { de vuelo }\end{array}$} & Grav & $1,304-1,319$ & $1,132-1,267$ & $1,005-1,007$ & $1,295-1,377$ & $0,130-0,135$ & $0,054-0,054$ \\
\hline & $655 \mathrm{rpm}$ & $0,209-0,222$ & $0,210-0,210$ & $0,204-0,204$ & $0,204-0,209$ & $0,063-0,063$ & $0,057-0,057$ \\
\hline & $690 \mathrm{rpm}$ & - & - & - & - & $0,167-0,168$ & $0,162-0,162$ \\
\hline & 842 rpm & $0,685-0,685$ & $0,667-0,678$ & $0,544-0,544$ & $0,539-0,540$ & $0,060-0,060$ & $0,054-0,054$ \\
\hline \multirow{3}{*}{$\begin{array}{c}\text { Sensibilidad } \\
\text { a las } \\
\text { rpm }\end{array}$} & $11 \mathrm{~Hz}$ & - & - & - & - & $0,174-0,176$ & $0,168-0,172$ \\
\hline & $12 \mathrm{~Hz}$ & $1,083-1,115$ & $1,056-1,058$ & $1,054-1,054$ & $1,091-1,098$ & $0,263-0,263$ & $0,196-0,196$ \\
\hline & $13 \mathrm{~Hz}$ & - & - & - & - & $0,195-0,198$ & $0,186-0,261$ \\
\hline \multirow{6}{*}{$\begin{array}{l}\text { Momento y } \\
\text { forma de } \\
\text { entrada de } \\
\text { la carga }\end{array}$} & $\operatorname{Cos} 15^{\circ}$ & $0,579-0,614$ & $0,487-0,488$ & $0,481-0,482$ & $0,480-0,480$ & $0,142-0,145$ & $0,139-0,181$ \\
\hline & $\operatorname{Cos} 30^{\circ}$ & $0,936-0,936$ & $0,603-0,604$ & $0,645-0,646$ & $0,998-0,998$ & $0,142-0,145$ & $0,139-0,181$ \\
\hline & $\operatorname{Cos} 45^{\circ}$ & $1,168-1,259$ & $1,181-1,260$ & $1,218-1,244$ & $1,252-1,259$ & $0,143-0,146$ & $0,139-0,181$ \\
\hline & $\operatorname{Lin} 15^{\circ}$ & - & - & - & - & $0,142-0,142$ & $0,139-0,181$ \\
\hline & $\operatorname{Lin} 30^{\circ}$ & $0,594-0,594$ & $0,521-0,560$ & $0,401-0,408$ & $0,399-0,400$ & $0,142-0,145$ & $0,139-0,181$ \\
\hline & $\operatorname{Lin} 45^{\circ}$ & $1,009-1,010$ & $0,958-1,010$ & $0,666-0,667$ & $0,703-0,703$ & $0,143-0,146$ & $0,139-0,181$ \\
\hline \multirow{10}{*}{$\begin{array}{l}\text { Sensibilidad } \\
\text { a la } \\
\text { rigidez } \\
\text { de la unión } \\
\text { bancada } \\
\text { ala }\end{array}$} & 0,75 & $1,111-1,206$ & $0,689-0,723$ & $0,444-0,557$ & $1,175-1,201$ & $0,142-0,142$ & $0,139-0,182$ \\
\hline & 0,6 & $0,507-0,518$ & $0,495-0,495$ & $0,447-0,447$ & $0,488-0,488$ & $0,142-0,142$ & $0,139-0,182$ \\
\hline & 0,5 & $0,665-0,666$ & $0,446-0,497$ & $0,400-0,427$ & $0,414-0,414$ & $0,142-0,142$ & $0,139-0,182$ \\
\hline & 0,33 & $0,739-0,750$ & $0,709-0,714$ & $0,445-0,716$ & $0,746-0,750$ & $0,141-0,141$ & $0,139-0,181$ \\
\hline & 0,25 & - & - & - & - & $0,141-0,141$ & $0,139-0,181$ \\
\hline & 0,2 & $0,591-0,591$ & $0,595-0,596$ & $0,551-0,598$ & $0,639-0,656$ & $0,134-0,154$ & $0,055-0,055$ \\
\hline & 0,1 & $0,644-0,648$ & $0,646-0,646$ & $0,554-0,554$ & $0,659-0,740$ & $0,135-0,157$ & $0,055-0,055$ \\
\hline & 0,05 & $0,993-0,993$ & $0,570-0,570$ & $0,637-0,638$ & $0,836-0,836$ & $0,061-0,061$ & $0,055-0,055$ \\
\hline & 0,02 & $0,588-0,589$ & $0,547-0,579$ & $0,594-0,620$ & $0,620-0,621$ & $0,062-0,063$ & $0,057-0,057$ \\
\hline & 0,01 & $0,395-0,400$ & $0,376-0,381$ & $0,329-0,335$ & $0,334-0,335$ & $0,091-0,095$ & $0,094-0,114$ \\
\hline \multirow{5}{*}{$\begin{array}{c}\text { Ratio } \\
\text { de pala } \\
\text { remanente }\end{array}$} & 0,01 & $0,605-0,707$ & $0,606-0,609$ & $0,443-0,617$ & $0,705-0,706$ & $0,142-0,142$ & $0,139-0,182$ \\
\hline & 0,2 & $1,227-1,227$ & $1,293-1,294$ & $0,956-0,956$ & $1,404-1,405$ & $0,146-0,150$ & $0,139-0,182$ \\
\hline & 0,4 & $0,814-0,876$ & $0,692-0,693$ & $0,779-0,782$ & $0,824-0,929$ & $0,151-0,151$ & $0,147-0,183$ \\
\hline & 0,6 & $1,514-1,514$ & $2,037-2,050$ & $2,050-2,053$ & $1,853-1,854$ & $0,336-0,336$ & $0,186-0,262$ \\
\hline & 0,8 & - & - & - & - & $0,644-0,695$ & $0,473-0,531$ \\
\hline \multirow{4}{*}{$\begin{array}{l}\text { Flexibilidad } \\
\text { del ala }\end{array}$} & $1,76 \mathrm{~Hz}$ & $0,574-0,574$ & $0,575-0,576$ & $0,620-0,770$ & $0,669-0,796$ & $0,148-0,154$ & $0,108-0,108$ \\
\hline & $1,68 \mathrm{~Hz}$ & $0,684-0,739$ & $0,883-0,890$ & $0,901-0,993$ & $0,907-0,943$ & $0,148-0,154$ & $0,108-0,108$ \\
\hline & $1,65 \mathrm{~Hz}$ & $0,575-0,575$ & $0,577-0,577$ & $0,620-0,620$ & $0,671-0,671$ & $0,148-0,154$ & $0,108-0,108$ \\
\hline & $1,57 \mathrm{~Hz}$ & $0,661-0,661$ & $0,666-0,666$ & $0,684-0,684$ & $0,746-0,782$ & $0,148-0,154$ & $0,108-0,108$ \\
\hline \multirow{5}{*}{$\begin{array}{c}\text { Factor de } \\
\text { amortiguamiento } \\
\text { estructural }\end{array}$} & 0,01 & $1,037-1,038$ & $0,775-0,783$ & $0,979-0,979$ & $1,061-1,062$ & $0,142-0,142$ & $0,139-0,182$ \\
\hline & 0,02 & $0,906-0,938$ & $0,743-0,748$ & $0,809-0,825$ & $1,015-1,016$ & $0,143-0,143$ & $0,139-0,182$ \\
\hline & 0,03 & $0,815-1,174$ & $0,660-0,700$ & $0,820-0,866$ & $0,882-0,996$ & $0,143-0,143$ & $0,139-0,182$ \\
\hline & 0,04 & $1,045-1,066$ & $0,688-0,696$ & $0,944-0,951$ & $0,958-1,066$ & $0,143-0,143$ & $0,139-0,182$ \\
\hline & 0,05 & $0,731-0,885$ & $0,698-0,734$ & $0,599-0,606$ & $0,815-0,822$ & $0,143-0,143$ & $0,139-0,182$ \\
\hline \multicolumn{2}{|l|}{ MEDIA } & $0,799-0,831$ & $0,731-0,748$ & $0,705-0,734$ & $0,808-0,835$ & $0,150-0,156$ & $0,125-0,149$ \\
\hline
\end{tabular}

Tabla 4.2: Intervalos de tiempos de fallos de los apoyos en los casos de carga 
rompa el apoyo trasero $\mathrm{Y}$ - luego el $\mathrm{Y}+\mathrm{y}$ luego de forma menos definida el apoyo superior $\mathrm{Y}+$, el apoyo lateral Y- y para terminar los apoyos laterales Y+y el apoyo superior Y-. Esta secuencia concuerda también con lo mostrado en los tiempos medios de rotura de la tabla 4.2. También en la figura 4.22 se muestran en qué número de ciclo se produce la rotura de cada uno de los apoyos.

\begin{tabular}{|c|c|c|c|c|c|c|}
\hline ORDEN & $\begin{array}{c}\text { Fallo apoyo } \\
\text { Lateral Y+ (s) }\end{array}$ & $\begin{array}{c}\text { Fallo apoyo } \\
\text { Lateral Y- (s) }\end{array}$ & $\begin{array}{c}\text { Fallo apoyo } \\
\text { Superior Y+ (s) }\end{array}$ & $\begin{array}{c}\text { Fallo apoyo } \\
\text { Superior Y- (s) }\end{array}$ & $\begin{array}{c}\text { Fallo apoyo } \\
\text { Trasero Y+ (s) }\end{array}$ & $\begin{array}{c}\text { Fallo apoyo } \\
\text { Trasero Y- (s) }\end{array}$ \\
\hline 1 & 0 & 0 & 0 & 0 & 1 & 44 \\
\hline 2 & 0 & 0 & 0 & 0 & 44 & 1 \\
\hline 3 & 6 & 11 & 14 & 5 & 0 & 0 \\
\hline 4 & 9 & 12 & 10 & 5 & 0 & 0 \\
\hline 5 & 10 & 12 & 10 & 4 & 0 & 0 \\
\hline 6 & 11 & 1 & 2 & 22 & 0 & 0 \\
\hline
\end{tabular}

Tabla 4.3: Número de veces que rompe cada apoyo en un orden especificado

En lo que respecta a las fuerzas en las condiciones de contorno se produce una superación sistemática de los límites admisibles en la dirección Y, en el caso de la dirección X se supera los límites en repetidas ocasiones aunque cuando lo hace es con valores muy similares al admisible. En este sentido cabe destacar que las cargas en los apoyos bajan mucho en ciertos grupos de casos de carga: rigidez de la unión bancada-ala, ratio remanente de pala y flexibilidad del ala.

En la figura 4.20 se muestran el número de elementos que han llegado a plastificar por caso de carga. Y en la figura 4.21 se muestra el ángulo en el que se produce el fallo dentro del plano de la hélice para cada apoyo. El color de la columna indica el orden, dentro de la secuencia de rotura, en la que se ha producido el fallo. Como se observa, salvo en los apoyos traseros no parecen existir ángulos más propensos que otros al fallo.

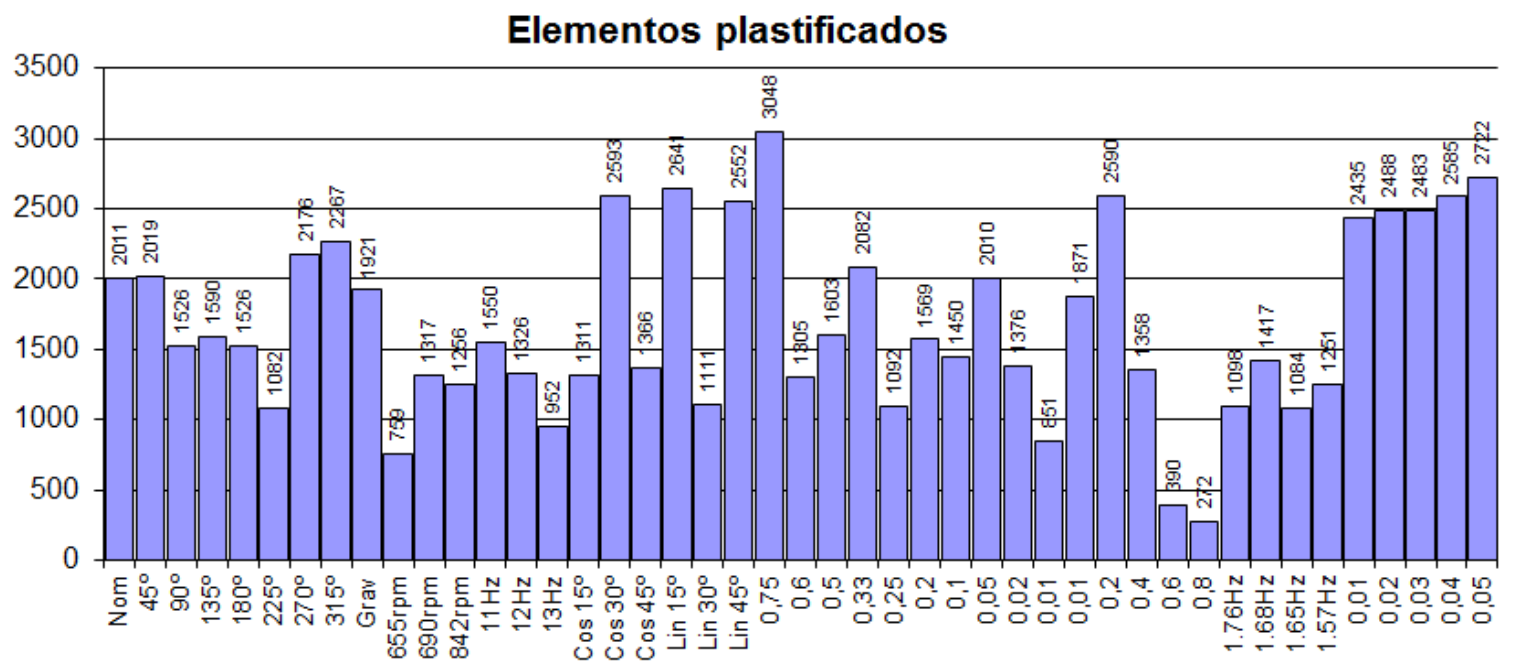

Figura 4.20: Elementos plastificados en los distintos casos de carga 

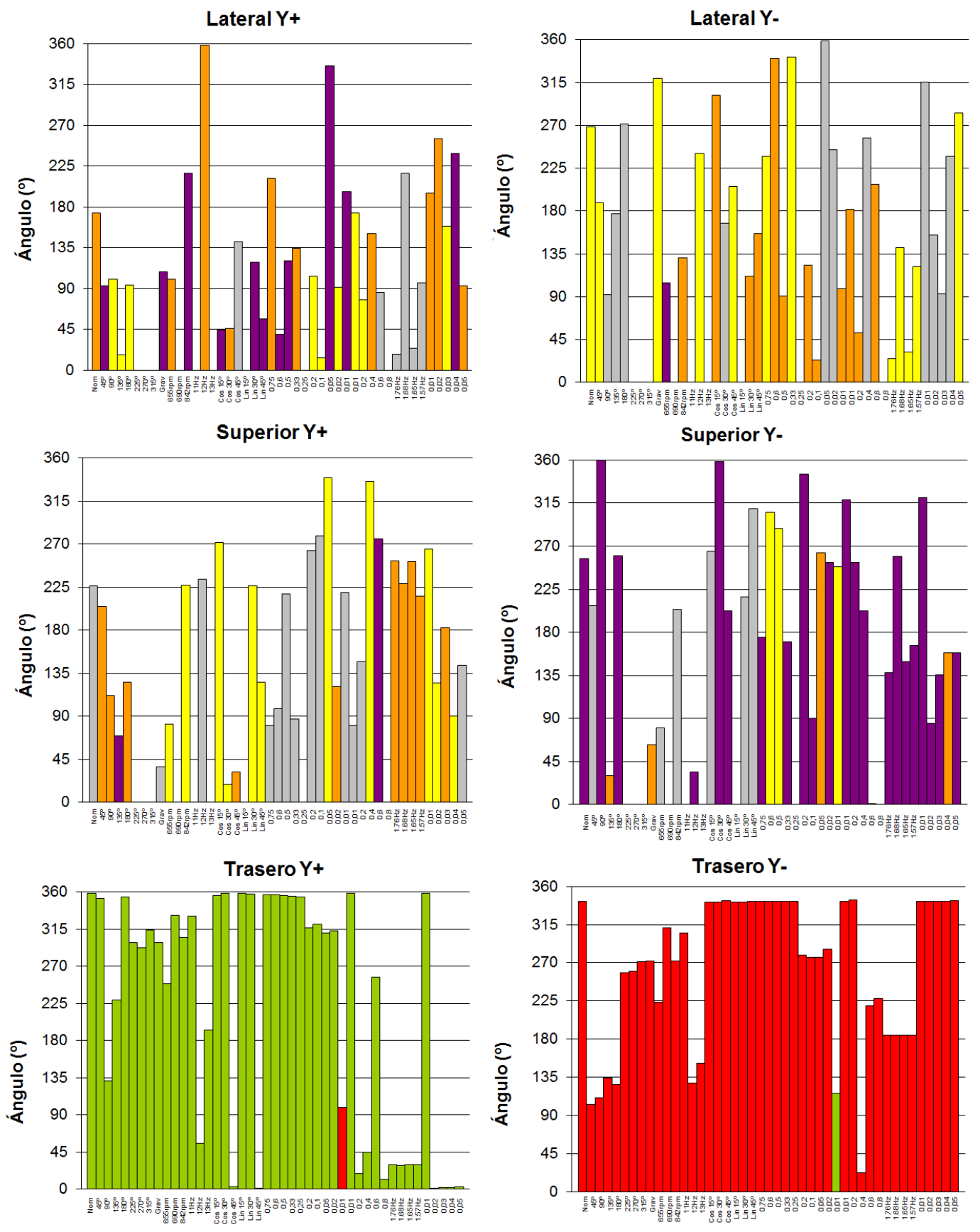

Figura 4.21: Ángulo y secuencia (rojo $1^{\circ}$, verde $2^{\circ}$, gris $3^{\circ}$, amarillo $4^{\circ}$, naranja $5^{\circ}$ y púrpura $6^{\circ}$ ) en los que se produce el fallo de los apoyos para cada caso de carga

Finalmente, en la tabla 4.4 y la figura 4.24 se muestra la energía que tiene el modelo en el momento que se produce la rotura del apoyo correspondiente para los distintos casos de carga. Como se observa la energía del modelo cuando se produce la rotura tiene una variabilidad muy grande alrededor de la media (desviación típica alta), sobre todo en los apoyos delanteros. 


\begin{tabular}{|c|c|c|c|c|c|c|c|}
\hline CASO DE CARGA & CASO & $\begin{array}{c}\text { Fallo apoyo } \\
\text { Lateral Y+ (s) }\end{array}$ & $\begin{array}{l}\text { Fallo apoyo } \\
\text { Lateral Y- (s) }\end{array}$ & $\begin{array}{c}\text { Fallo apoyo } \\
\text { Superior Y+(s) }\end{array}$ & $\begin{array}{c}\text { Fallo apoyo } \\
\text { Superior Y- (s) }\end{array}$ & $\begin{array}{c}\text { Fallo apoyo } \\
\text { Trasero Y+ (s) }\end{array}$ & $\begin{array}{l}\text { Fallo apoyo } \\
\text { Trasero Y- (s) }\end{array}$ \\
\hline \multirow[t]{3}{*}{ NOMINAL } & Nom & $4,36 E+05$ & $4,05 E+05$ & $1,88 E+05$ & $4,45 E+05$ & $5,49 E+04$ & $5,28 E+04$ \\
\hline & $45^{\circ}$ & $5,90 E+05$ & $5,71 E+05$ & $5,72 E+05$ & $3,88 E+05$ & $4,12 E+04$ & $4,91 E+04$ \\
\hline & $90^{\circ}$ & $3,10 E+05$ & $3,08 E+05$ & $3,13 E+05$ & $3,37 E+05$ & $4,62 E+04$ & $5,01 E+04$ \\
\hline \multirow{5}{*}{$\begin{array}{l}\text { Posición } \\
\text { angular }\end{array}$} & $135^{\circ}$ & $4,75 E+05$ & $4,29 E+05$ & $4,92 E+05$ & $4,81 E+05$ & $5,40 E+04$ & $4,25 E+04$ \\
\hline & $180^{\circ}$ & $5,80 E+05$ & $4,52 E+05$ & $5,82 E+05$ & $5,74 E+05$ & $8,97 E+04$ & $4,81 E+04$ \\
\hline & $225^{\circ}$ & & & & & $4,98 E+04$ & $5,14 E+04$ \\
\hline & $270^{\circ}$ & & & & & $5,71 E+04$ & $5,40 E+04$ \\
\hline & $315^{\circ}$ & & & & & $4,83 E+04$ & $4,59 E+04$ \\
\hline \multirow{4}{*}{$\begin{array}{c}\text { Puntos } \\
\text { de la } \\
\text { envolvente } \\
\text { de vuelo }\end{array}$} & Grav & $5,67 E+05$ & $4,17 E+05$ & $3,63 E+05$ & $5,46 E+05$ & $4,69 E+04$ & $3,69 E+04$ \\
\hline & $655 \mathrm{rpm}$ & $1,72 E+05$ & $1,73 E+05$ & $1,68 E+05$ & $1,68 E+05$ & $4,16 E+04$ & $3,67 E+04$ \\
\hline & $690 \mathrm{rpm}$ & & & & & $7,78 E+04$ & $7,93 E+04$ \\
\hline & $842 \mathrm{rpm}$ & $3,38 E+05$ & $3,24 E+05$ & $2,59 E+05$ & $2,58 E+05$ & $3,81 E+04$ & $3,67 E+04$ \\
\hline \multirow{3}{*}{$\begin{array}{c}\text { Sensibilidad } \\
\text { a las } \\
\text { rpm } \\
\end{array}$} & $11 \mathrm{~Hz}$ & & & & & $6,29 E+04$ & $6,35 E+04$ \\
\hline & $12 \mathrm{~Hz}$ & $3,32 E+05$ & $3,11 E+05$ & $3,13 E+05$ & $3,31 E+05$ & $3,92 E+04$ & $3,18 E+04$ \\
\hline & $13 \mathrm{~Hz}$ & & & & & $2,96 E+04$ & $2,54 E+04$ \\
\hline \multirow{6}{*}{$\begin{array}{l}\text { Momento y } \\
\text { forma de } \\
\text { entrada de } \\
\text { la carga }\end{array}$} & $\operatorname{Cos} 15^{\circ}$ & $2,45 E+05$ & $2,14 E+05$ & $2,18 E+05$ & $2,19 E+05$ & $5,27 E+04$ & $5,08 E+04$ \\
\hline & $\operatorname{Cos} 30^{\circ}$ & $7,43 E+05$ & $4,27 E+05$ & $4,97 E+05$ & $9,24 E+05$ & $5,01 E+04$ & $4,81 E+04$ \\
\hline & $\operatorname{Cos} 45^{\circ}$ & $3,64 E+05$ & $4,05 E+05$ & $4,19 E+05$ & $4,75 E+05$ & $4,70 E+04$ & $4,51 E+04$ \\
\hline & $\operatorname{Lin} 15^{\circ}$ & & & & & $5,34 E+04$ & $5,13 E+04$ \\
\hline & $\operatorname{Lin} 30^{\circ}$ & $3,52 E+05$ & $3,33 E+05$ & $2,02 E+05$ & $2,00 E+05$ & $5,15 E+04$ & $4,94 E+04$ \\
\hline & $\operatorname{Lin} 45^{\circ}$ & $6,35 E+05$ & $5,75 E+05$ & $3,76 E+05$ & $4,18 E+05$ & $5,36 E+04$ & $5,12 E+04$ \\
\hline \multirow{10}{*}{$\begin{array}{l}\text { Sensibilidad } \\
\text { a la } \\
\text { rigidez } \\
\text { de la unión } \\
\text { bancada } \\
\text { ala }\end{array}$} & 0,75 & $9,52 E+05$ & $4,74 E+05$ & $2,39 E+05$ & $1,01 E+06$ & $5,51 E+04$ & $5,30 E+04$ \\
\hline & 0,6 & $3,10 E+05$ & $3,02 E+05$ & $2,56 E+05$ & $3,00 E+05$ & $5,52 E+04$ & $5,34 E+04$ \\
\hline & 0,5 & $3,49 E+05$ & $2,44 E+05$ & $1,85 E+05$ & $2,03 E+05$ & $5,54 E+04$ & $5,35 E+04$ \\
\hline & 0,33 & $5,60 E+05$ & $5,09 E+05$ & $2,35 E+05$ & $5,70 E+05$ & $5,61 E+04$ & $5,43 E+04$ \\
\hline & 0,25 & & & & & $5,66 E+04$ & $5,48 E+04$ \\
\hline & 0,2 & $2,85 E+05$ & $2,88 E+05$ & $2,68 E+05$ & $3,62 E+05$ & $5,04 E+04$ & $3,96 E+04$ \\
\hline & 0,1 & $3,19 E+05$ & $3,24 E+05$ & $3,04 E+05$ & $3,63 E+05$ & $5,34 E+04$ & $3,90 E+04$ \\
\hline & 0,05 & $5,72 E+05$ & $2,38 E+05$ & $2,84 E+05$ & $3,60 E+05$ & $4,14 E+04$ & $3,88 E+04$ \\
\hline & 0,02 & $3,12 E+05$ & $2,60 E+05$ & $3,22 E+05$ & $3,48 E+05$ & $4,12 E+04$ & $3,97 E+04$ \\
\hline & 0,01 & $3,26 E+05$ & $2,97 E+05$ & $3,07 E+05$ & $3,13 E+05$ & $4,80 E+04$ & $4,61 E+04$ \\
\hline \multirow{5}{*}{$\begin{array}{l}\text { Ratio } \\
\text { de pala } \\
\text { remanente }\end{array}$} & 0,01 & $4,21 E+05$ & $4,22 E+05$ & $2,29 E+05$ & $4,03 E+05$ & $5,49 E+04$ & $5,28 E+04$ \\
\hline & 0,2 & $6,94 E+05$ & $7,21 E+05$ & $4,97 E+05$ & $7,77 E+05$ & $4,82 E+04$ & $4,58 E+04$ \\
\hline & 0,4 & $2,66 E+05$ & $2,07 E+05$ & $2,43 E+05$ & $2,72 E+05$ & $3,02 E+04$ & $3,08 E+04$ \\
\hline & 0,6 & $8,21 E+04$ & $2,21 E+05$ & $2,15 E+05$ & $1,75 E+05$ & $2,78 E+04$ & $1,27 E+04$ \\
\hline & 0,8 & & & & & $1,05 E+04$ & $8,71 E+03$ \\
\hline \multirow{4}{*}{$\begin{array}{c}\text { Flexibilidad } \\
\text { del ala }\end{array}$} & $1,76 \mathrm{~Hz}$ & $2,36 E+05$ & $2,35 E+05$ & $2,72 E+05$ & $3,34 E+05$ & $6,73 E+04$ & $6,19 E+04$ \\
\hline & $1,68 \mathrm{~Hz}$ & $3,09 E+05$ & $4,80 E+05$ & $5,12 E+05$ & $5,28 E+05$ & $6,73 E+04$ & $6,20 E+04$ \\
\hline & $1,65 \mathrm{~Hz}$ & $2,33 E+05$ & $2,32 E+05$ & $2,70 E+05$ & $3,17 E+05$ & $6,73 E+04$ & $6,20 E+04$ \\
\hline & $1,57 \mathrm{~Hz}$ & $3,66 E+05$ & $3,81 E+05$ & $4,11 E+05$ & $4,49 E+05$ & $6,73 E+04$ & $6,20 E+04$ \\
\hline \multirow{5}{*}{$\begin{array}{c}\text { Factor de } \\
\text { amortiguamiento } \\
\text { estructural }\end{array}$} & 0,01 & $7,02 E+05$ & $4,75 E+05$ & $6,43 E+05$ & $7,49 E+05$ & $5,49 E+04$ & $5,27 E+04$ \\
\hline & 0,02 & $5,87 E+05$ & $4,48 E+05$ & $5,18 E+05$ & $6,34 E+05$ & $5,51 E+04$ & $5,26 E+04$ \\
\hline & 0,03 & $5,63 E+05$ & $4,00 E+05$ & $5,64 E+05$ & $5,72 E+05$ & $5,50 E+04$ & $5,26 E+04$ \\
\hline & 0,04 & $7,79 E+05$ & $4,54 E+05$ & $5,71 E+05$ & $6,12 E+05$ & $5,49 E+04$ & $5,25 E+04$ \\
\hline & 0,05 & $6,02 E+05$ & $5,45 E+05$ & $4,14 E+05$ & $7,98 E+05$ & $5,49 E+04$ & $5,24 E+04$ \\
\hline \multicolumn{2}{|l|}{ MEDIA } & $4,43 E+05$ & $3,75 E+05$ & $3,53 E+05$ & $4,50 E+05$ & $5,14 E+04$ & $4,74 E+04$ \\
\hline
\end{tabular}

Tabla 4.4: Energía $[\mathrm{J}]$ del modelo cuando rompe el apoyo para todos los casos de carga 


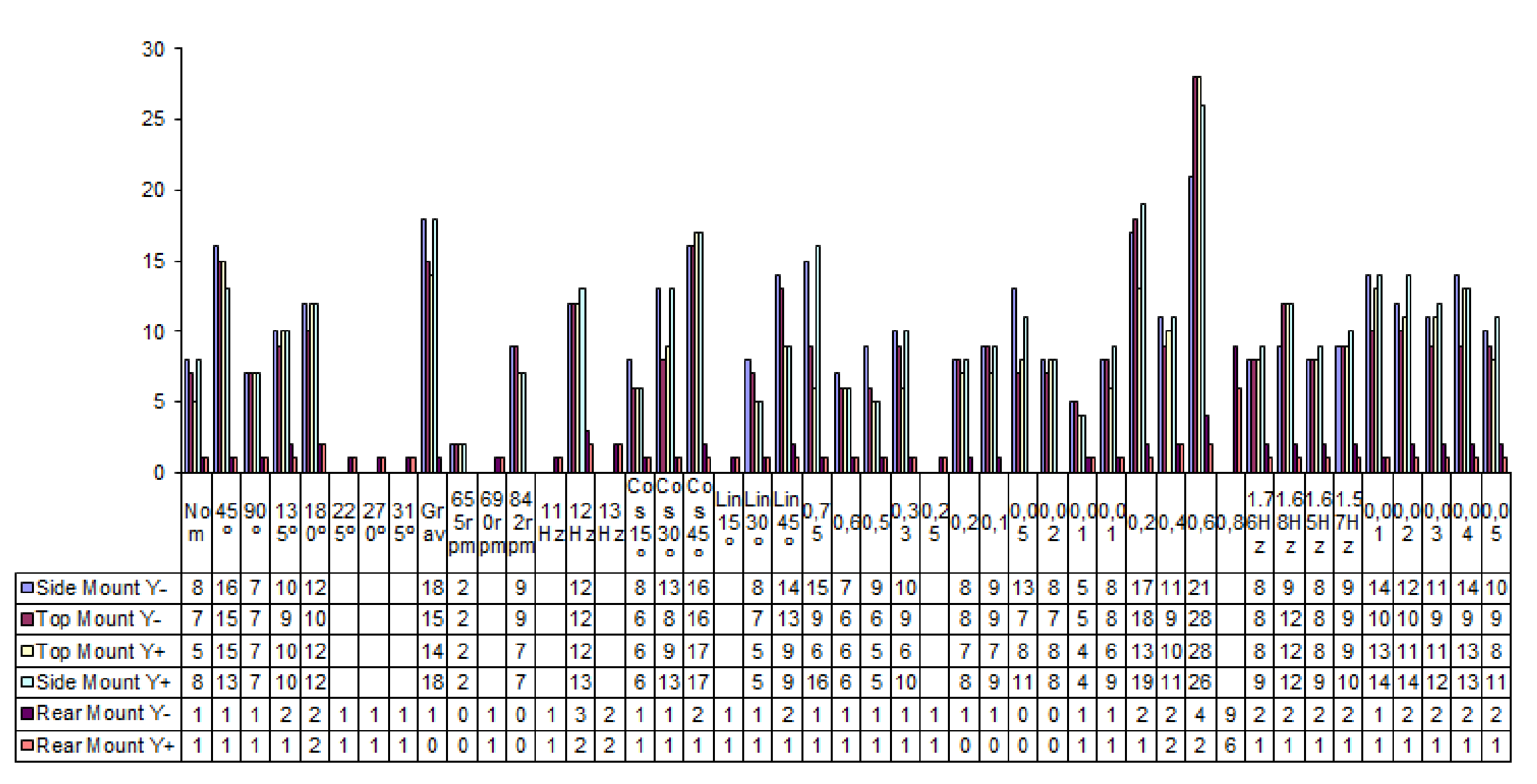

Figura 4.22: Número de ciclos en los distintos casos de carga 


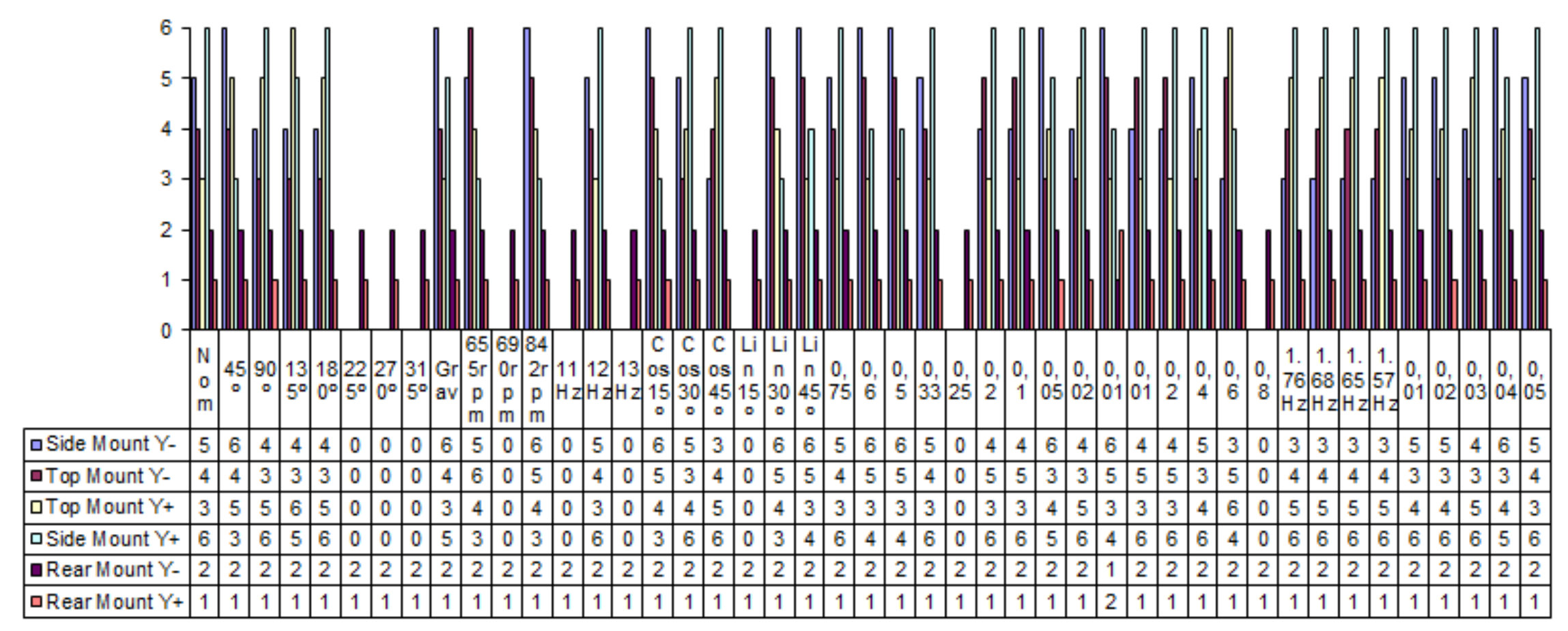

Figura 4.23: Secuencia de fallo en los distintos casos de carga 


\section{Energía del modelo cuando fallan los apoyos}

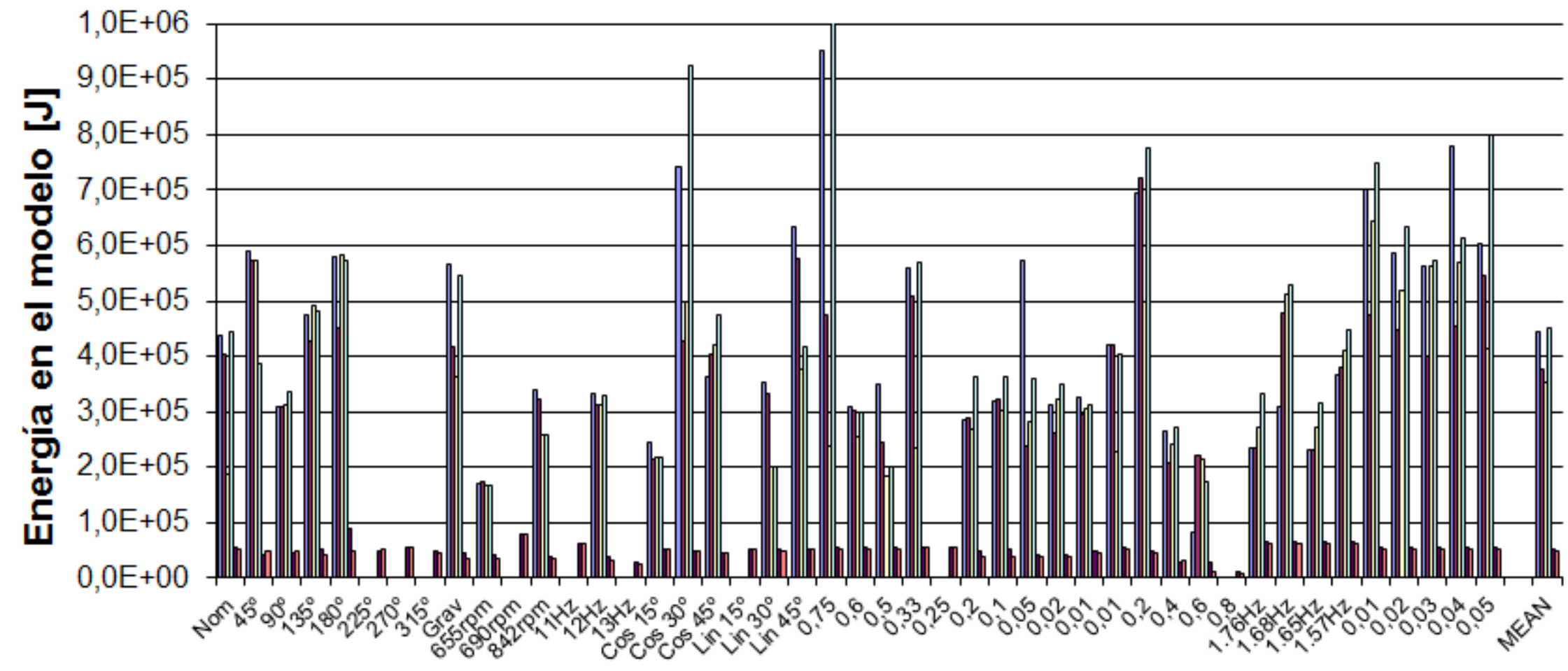

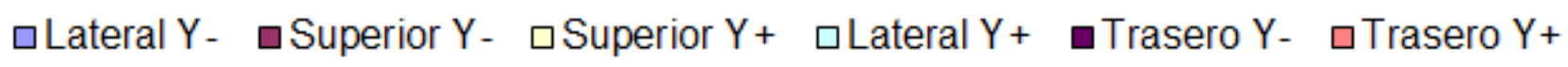




\subsubsection{Resumen resultados por grupos de caso de carga}

Finalmente, una vez mostrados gran parte de los resultados obtenidos y como adelanto a lo que se trata más adelante en la sección 6.1.1, se describen una serie de conclusiones particulares extraídas de los grupos de casos de carga que se han analizado:

- Posición angular: Se observa que el tiempo necesario para que el motor se desprenda es mayor cuando los valores de $\theta$ se encuentran entre $225^{\circ}$ y $45^{\circ}$, lo mismo sucede con los elementos plastificados.

- Puntos de la envolvente de vuelo: El tiempo que se necesita para desprender el motor a velocidad $V_{b}$ a nivel del mar es menor que aplicando sólo la gravedad.

- Sensibilidad a las rpm: En este caso como se ha mencionado las ecuaciones muestran que las fuerzas y momentos decrecen cuando la frecuencia decrece. Por este motivo se podría pensar que este decremento produciría otro decremento en el número de elementos plastificados o un incremento en el tiempo que llevaría el desprendimiento del motor. Sin embargo, éste no es el caso y no se puede asegurar. Por el contrario los resultados se muestran muy dependientes a la cercanía o lejanía de la frecuencia de la hélice a los modos propios de la estructura. La velocidad de absorción es sensiblemente mayor a $12 \mathrm{~Hz}$ que a $11 \mathrm{~Hz}$ ó $13 \mathrm{~Hz}$.

- Sensibilidad a la rigidez de la unión bancada-ala: Los resultados no muestran patrones claros de comportamiento cuando se incrementa o disminuye la rigidez de la unión. Lo que sí es evidente es que la variación de la rigidez de la unión bancada-ala varía los valores de frecuencia de los modos propios de todo el sistema y, por lo tanto, sí que existe una cierta sensibilidad en los resultados. De hecho, cuando los ratios de rigidez están entre $0.25 \mathrm{y}$ 0.75 el modelo se muestra más imperturbable a las cargas aplicadas y requiere un mayor tiempo llegar a niveles de energía suficientes para desprender el motor.

- Momento y forma de introducción de las fuerzas: Los resultados muestran la variación inicial de la carga modifica en cierta medida los resultados. Por ejemplo el tiempo que se necesita para desprender el motor del ala es mayor si la forma de introducir la carga es más suave.

- Tamaño de la pala perdida: Como se indicaba en las ecuaciones las fuerzas y momentos disminuyen cuando el ratio aumenta y en este caso los resultados se muestran como inicialmente se podían esperar. El tiempo necesario para desprender el motor del ala es mayor cuando el ratio es mayor. El número de elementos plastificados es menor cuando el ratio es mayor. La velocidad de absorción de energía es menor cuando el ratio es mayor y lo mismo sucede con las fuerzas que llegan al ala. 
- Amortiguamiento estructural: Los resultados encontrados no son como a priori se podían pensar, por lo menos para los valores que se han estudiado. El número de elementos plastificados es menor cuando el amortiguamiento también lo es. Y el tiempo necesario para que el motor se suelte de la bancada es mayor cuando decrece el amortiguamiento estructural.

- Flexibilidad del ala: Este caso tiene similitudes con el caso de la variación de la rigidez de la unión bancada-ala y los resultados tampoco muestran patrones claros de comportamiento cuando se varía la flexibilidad del ala. Un cambio en la flexibilidad del ala cambia también los modos propios de todo el modelo. Esto hace que pueda cambiar la sensibilidad del sistema ante las cargas aplicadas. Se ha encontrado que flexibilidades de ala de $1.57 \mathrm{~Hz}$ ó $1.68 \mathrm{~Hz}$ hacen al modelo menos sensible a las cargas aplicadas, y lleva más tiempo llegar a altos niveles de energía. 


\section{ANÁlisis ALEATORIO DE LA PÉRDIDA DE PALA}

\section{Índice}

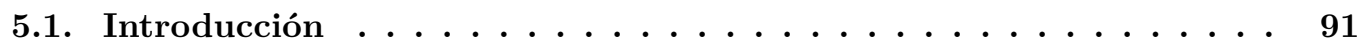

5.1.1. Ejemplo: Problema con variables aleatorias . . . . . . . . . . . . . 92

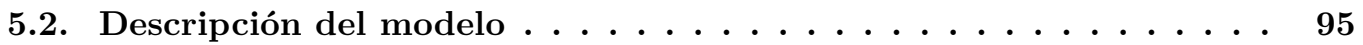

5.2.1. Reducción del modelo . . . . . . . . . . . . . . . . . . . . 95

5.2 .2$. La bancada . . . . . . . . . . . . . . . . . . . . . . . . 98

5.2.2.1. Unión motor-bancada . . . . . . . . . . . . . . . 98

5.2.2.2. Unión bancada-ala . . . . . . . . . . . . . . . . . . . 98

5.2.3. Consideraciones del análisis FEM; método implícito y explícito; paso de

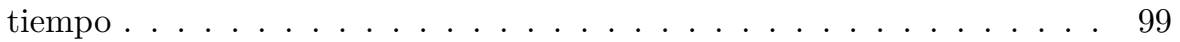

5.2.3.1. Esquema de análisis . . . . . . . . . . . . . . . 100

5.2.3.2. Niveles de energía . . . . . . . . . . . . . . . . . . 101

5.2.3.3. Amortiguamiento estructural . . . . . . . . . . . . . . 102

5.2.4. Cargas . . . . . . . . . . . . . . . . . . . . . . . . 103

5.3. Casos de carga analizados . . . . . . . . . . . . . 103

5.3.1. Técnica del método de Monte Carlo (MCT) . . . . . . . . . . . . . . 103

5.3.2. Configuración de los parámetros de entrada . . . . . . . . . . . . . . . . 104

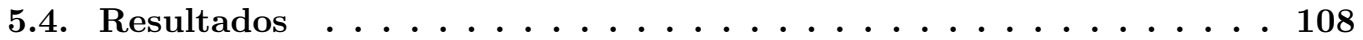

5.4.1. Resultados preliminares de variaciones individuales con el modelo sólo motor . . . . . . . . . . . . . . . . . . . 108

5.4.2. Resultados de variaciones individuales de los parámetros de entrada . . 112

5.4.3. Interacción de todos los parámetros . . . . . . . . . . . . . . . 121

5.4.4. Resultados de la unión bancada-ala . . . . . . . . . . . . . . . . . . 129

5.4.5. Resultados de la secuencia de fallo . . . . . . . . . . . . . . 130

\subsection{Introducción}

En los capítulos anteriores se ha desarrollado un método analítico por el método de los elementos finitos para predecir la respuesta no-lineal transitoria de todo un sistema motor, incluida 
la bancada, por una pérdida de pala. Esta respuesta depende especialmente de la condición de vuelo en la que se produzca, la frecuencia a la que esté la hélice en ese momento, el tamaño de pala perdida, el ángulo en el que se produce la rotura y la manera en que se pierde (instantánea o de alguna otra manera). Además, la flexibilidad del ala, la rigidez de la unión bancada-ala y el amortiguamiento estructural del sistema son también parámetros importantes para el fenómeno. Sin embargo, la hipótesis de considerar valores deterministas para algunos parámetros es difícil de mantener. Algunas partes de los dispositivos de las uniones se rompen nada más producirse la pérdida de pala (especialmente los dispositivos elastoméricos), y los puntos o valores de rotura describen unas fluctuaciones no deterministas. Por lo tanto es determinante y más realista atacar el problema desde un punto de vista estocástico. Sin embargo, la gran cantidad de variables relevantes en el problema hacen casi imposible realizar un estudio detallado de todo el sistema.

A continuación, se muestra una serie de simulaciones por el método de Monte Carlo para analizar en detalle la secuencia de rotura de los elastómeros. Esta metodología se explica en el apartado 5.3.1 y es capaz de realizar el análisis con cualquier variación en los parámetros de entrada.

El capítulo se puede considerar continuación del anterior y cuya introducción, apartado 4.1 y 4.2 donde se introduce y se describe el fenómeno de la pérdida de pala, es aplicable a éste. Este capítulo está organizado de la siguiente manera. En el apartado 5.1.1 se muestra con un sencillo ejemplo la técnica que se ha introducido y que más adelante se utilizará para el fenómeno principal. El apartado 5.2 y 5.3 describe el modelo utilizado para simular el suceso, así como la filosofía de análisis, decisiones tomadas y casos de carga analizados. Finalmente, el apartado 5.4 muestra los resultados y en el apartado 6.1.3 se resumen las conclusiones principales. Además, en el apartado B.2 se describe cómo y de qué manera se ha llevado a cabo el flujo de integración en el software utilizado.

\subsubsection{Ejemplo: Problema con variables aleatorias}

Si se retoma el ejemplo de la viga en 3.3 .2 y se le aplican $P_{0}=100[\mathrm{~kg}]$ de peso en la punta de manera estática.

Se recuerda que las propiedades iniciales eran: viga cuadrada maciza lado $b_{0}=0,1 \mathrm{~m}$ y $h_{0}=0,1[\mathrm{~m}], E_{0}=70000[\mathrm{MPa}]$ módulo de Young de la viga, $L_{0}=10[\mathrm{~m}]$ longitud inicial de la viga.

Si ahora se consideran dos criterios de fallo: desplazamiento en punta menor a $a d m_{1}=0,6$ [m] y el esfuerzo máximo debe ser menor a $a d m_{2}=115$ [MPa]. Entonces, para que no falle ninguno de los criterios se deben cumplir las ecuaciones 5.1.1.

$$
\begin{aligned}
& f_{1}(X)=\frac{4 P L^{3}}{E b h^{3}}-a d m_{1} \leq 0 \\
& f_{2}(X)=\frac{12 P L}{b h^{2}}-a d m_{2} \leq 0
\end{aligned}
$$


Se tiene que $f_{1}=-0,04[\mathrm{~m}]$ y $f_{2}=3[\mathrm{MPa}]$ por lo que al haber un valor mayor que cero la viga fallaría y, en este caso, solamente por superar el máximo esfuerzo admisible.

Sin embargo, en la realidad las variables que representan magnitudes físicas no son siempre exactas y sucede que la fabricación no es siempre igual. Por este motivo, el admisible de esfuerzo máximo puede variar, a veces sucede que la estructura sometida a esos niveles de esfuerzo aguanta un poco más y otras menos.

Supongamos que este esfuerzo admisible sigue una normal de media $\mu=115$ [MPa] con una desviación típica de un \%10 de su valor $\sigma=11,5[\mathrm{MPa}]$ medido por el fabricante. Entonces, resulta que la probabilidad de que falle por superar el esfuerzo admisible ya no es el $100 \%$, es el $59 \%$, que es exactamente la probabilidad de la distribución de que el admisible del material tenga un valor menor a $117,72[\mathrm{MPa}]$.

Y si resultara también que el fabricante suministrara un aluminio en el que módulo de Young también variara, por ejemplo, según una distribución normal de media $E=70000$ [MPa] con una desviación típica de un $3 \%$ de su valor. Entonces, el admisible de desplazamiento que nunca se alcanzaba con las cargas aplicadas empezaría a alcanzarse con una probabilidad de $1,4 \%$. En el caso de que la desviación típica fuera un $5 \%$ el valor sería de $9,4 \%$, y si fuera de un $10 \%$ sería $25,6 \%$, que son exactamente las probabilidades de las distribuciones de que el módulo de Young tenga un valor menor a $65400[\mathrm{MPa}]$.

Si observamos en detalle las ecuaciones, habría variables que afectarían a las dos probabilidades de fallo, sin embargo, las variables hasta ahora escogidas: variar $E$ (un $3 \%$ ) sólo afecta a $f_{1}$, y variar $a d m_{2}$ (un $10 \%$ ) sólo afecta a $f_{2}$, como se puede ver en la figura 5.1. Las líneas verdes muestran los valores de $E=65400[\mathrm{MPa}]$ y $a d m_{2}=117,72[\mathrm{MPa}]$, que hacen $f_{1}=0$ y $f_{2}=0$ respectivamente.

Estas variaciones realizadas sobre estas variables se podría extender a otras y ver las influencias que tienen en los criterios de fallo, de hecho, en este ejemplo las demás variables influirían en ambos criterios, como se puede ver en la figura 5.1.

Si finalmente se decide variar las restantes variables: h, b, L y P de forma uniforme entre su valor nominal y un $\pm 10 \%$, y $E_{0}$ y $a d m_{2}$ según una distribución normal con un $\pm 10 \%$ de desviación típica, queda una probabilidad de fallo del 41,3\% para el criterio de desplazamiento máximo admisible y un 55,8\% para el criterio de máximo esfuerzo admisible. La figura que muestra las variables de entrada frente a las de salida (para $10^{6}$ simulaciones) es la figura 5.2, también muestra las relaciones entre las propias variables de entrada y las propias variables de salida. Se ha marcado con línea roja los valores de $f_{1}=0$ y $f_{2}=0$ que son la frontera entre el fallo y no fallo de cada uno de los criterios. Esta figura proporciona unos datos muy útiles de relaciones entre parámetros. Por ejemplo, si el módulo de Young crece, la probabilidad de que el criterio de máximo desplazamiento se cumpla es mayor, llegando casi al $100 \%$ para un módulo de $E \geq 90$ [GPa] (sean cual sean los demás parámetros), y si el $a d m_{2} \geq 150$ [MPa] también da igual cuales sean los demás parámetros que la viga cumple con el criterio de máximo esfuerzo. 


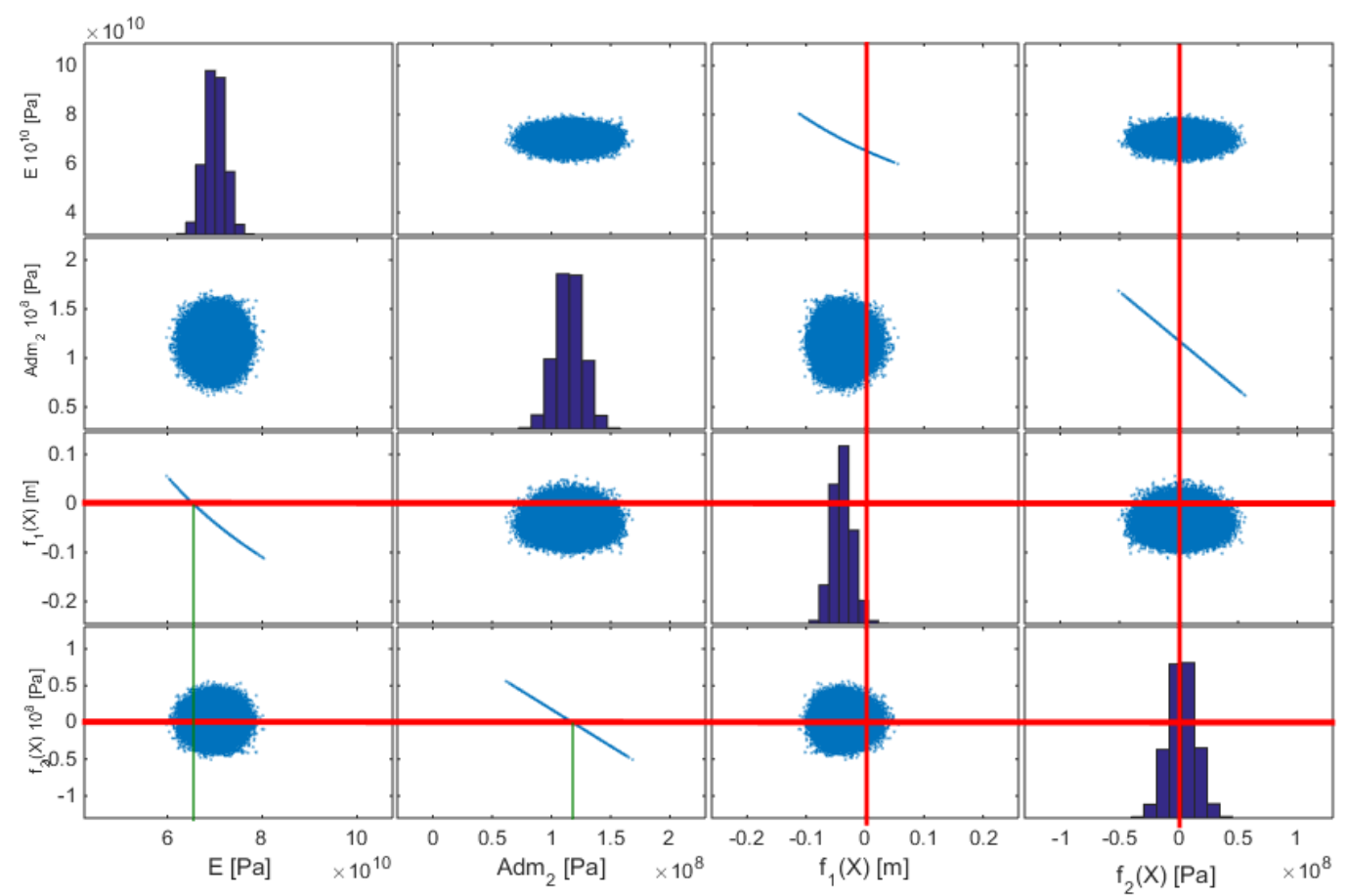

Figura 5.1: Correlaciones entre las variables $E, a d m_{2}, f_{1}$ y $f_{2}$

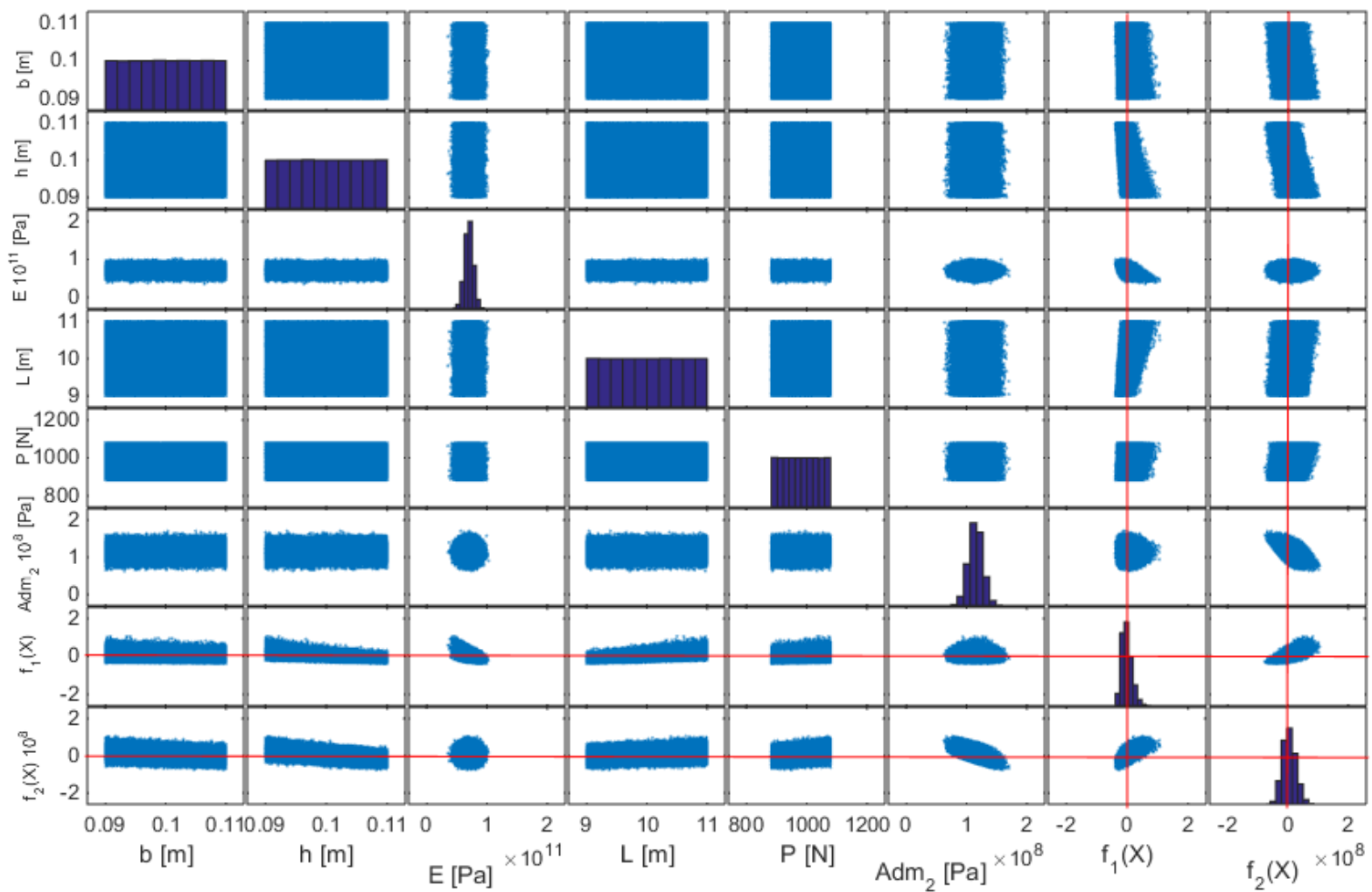

Figura 5.2: Histogramas y correlaciones entre variables de entrada y salida 


\subsection{Descripción del modelo}

Para analizar y simular el fenómeno de la pérdida de pala, se ha escogido un caso representativo, el mismo turbohélice de transporte militar pesado que en el capítulo 4 . Se puede ver una descripción más detallada en el apartado 4.3. Cabe recordar que un aspecto principal de los modelos desarrollados es que tratan de reproducir exactamente el comportamiento dinámico del motor mediante su masa e inercia. Por otra parte, los modos propios del modelo deben ser idénticos a los de la estructura real. En lo que respecta a la modelización de los motores el modelo FEM del capítulo anterior y éste, que se ha simplificado lo máximo posible (ver apartado 5.2.1), mantienen unos autovalores y unos autovectores muy parecidos para los primeros modos como se puede ver en la tabla 5.1.

\begin{tabular}{|c|c|c|}
\hline Modo & \% Error en la frecuencia & Autovector \\
\hline $1^{\circ}$ & 5,05 & Translación según eje Y combinado con rotación en plano XY \\
\hline $2^{\circ}$ & 7,50 & Translación según eje Z combinado con rotación en plano XZ \\
\hline $3^{o}$ & 8,23 & Translación según eje X combinado con rotación en plano XY \\
\hline $4^{o}$ & 9,99 & Translación según eje X combinado con rotación en plano XY \\
\hline
\end{tabular}

Tabla 5.1: Comparación de los modos propios entre modelos

Como ya se ha comentado la simplificación es fundamental para poder aplicar luego la técnica de Monte Carlo, se trata de un fenómeno con gran cantidad de variables relevantes y necesita de una reducción máxima del modelo manteniendo la información necesaria y suficiente de la estructura.

Aparte del modelo del motor, muchos de los esfuerzos se centran en el estudio de la rigidez del sistema de unión del motor al ala. Como ya se ha mencionado en el capítulo 4 la optimización del diseño del material elastomérico es esencial. Este problema no lineal debe considerar los valores de rotura de los dispositivos elastoméricos además de su rigidez no lineal. Sobre todo porque los criterios de fallo de estas partes de la estructura determinan el comportamiento del sistema. Las variaciones en el sistema de unión debido a un fallo modifican la dinámica del sistema y, como consecuencia, el momento y la secuencia de fallo de los dispositivos elastoméricos. Además, las propiedades de la bancada se tienen en cuenta como se indica en el apartado 5.2.1 y, consecuentemente, el modelo es capaz de reproducir el fenómeno desde la hélice hasta el ala. Finalmente, se ha introducido amortiguamiento estructural al sistema. La figura 5.3 resume lo anteriormente descrito.

\subsubsection{Reducción del modelo}

Para conseguir reducir el número de variables en este caso de estudio se ha desarrollado un modelo más simplificado manteniendo el comportamiento cualitativo del sistema. 


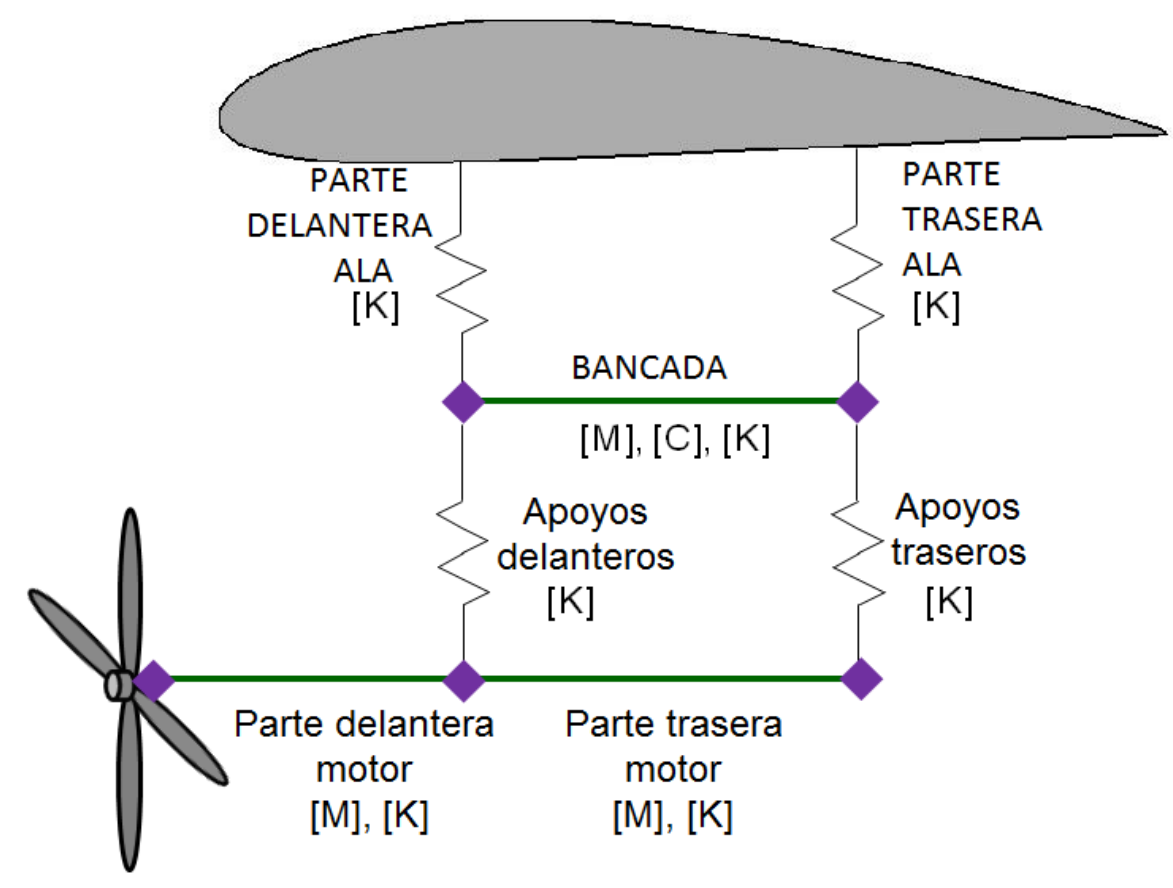

Figura 5.3: Esquema del modelo desde la hélice hasta el ala

La técnica utilizada para la reducción del modelo se ha basado en los superelementos (o reducción de Craig-Bampton) que permiten descomponer grandes modelos de elementos finitos en pequeñas subestructuras de elementos finitos como se ha explicado en el apartado 2.4. Así, en vez de resolver cada vez el modelo completo se utilizan estas subestructuras reducidas o superelementos, recaracterizando grandes modelos de elementos finitos en matrices de masa y de rigidez relativamente pequeñas. Estas matrices contienen las informaciones sobre los modos fundamentales para frecuencias bajas de la estructura.

Esta técnica hace uso de puntos interiores y de puntos exteriores (puntos interfaz) y consiste en combinar el movimiento de los grados de libertad de los puntos exteriores con los modos de la estructura reducida suponiendo que los demás puntos de interfaz están fijos. Para más información acerca de la técnica se puede consultar en los manuales MSC (2005), una aplicación práctica también se puede ver en Bah et al. (2003).

En este caso todo el motor se ha modelado mediante matrices de rigidez y de masa, teniendo especial atención al comportamiento estructural del motor, especialmente en la zona cercana a los apoyos tanto delantero como trasero donde se han incluido dos puntos de interfaz. Y para tener un grado mayor de precisión en el modelo se incluyen los grados de libertad en el punto de aplicación de la carga (ver figura 5.3). Finalmente, las propiedades de la bancada también se han incorporado con esta misma técnica y, otra vez, teniendo especial atención en la zona cercana a los apoyos a través de otros dos puntos. Estos puntos además de unir con el motor también unen con el ala para poder así reproducir el comportamiento hasta el ala. 
Cabe destacar diferencias entre el modelo de elementos finitos tratado en el capítulo 4 y el utilizado en este capítulo: las posiciones de los elastómeros se han trasladado desde sus posiciones al punto central con el mismo valor en $\mathrm{X}$ y, por lo tanto, ambos modelos serían equivalentes si existiera el mismo desplazamiento entre el origen y el destino de las zonas desplazadas (estructura infinitamente rígida entre los puntos), también se han trasladado los apoyos con el ala a un punto central y, de igual manera, serían equivalentes si hubiera los mismos desplazamientos en unos y otros puntos (estructura infinitamente rígida entre los puntos), siempre teniendo en cuenta los grados de libertad restringidos en cada uno. En este punto hay que señalar que el apoyo no es totalmente isostático y, por lo tanto ya hay una dependencia de la rigidez de la zona. Estas simplificaciones se pueden ver en la figura 5.4 y 5.5.

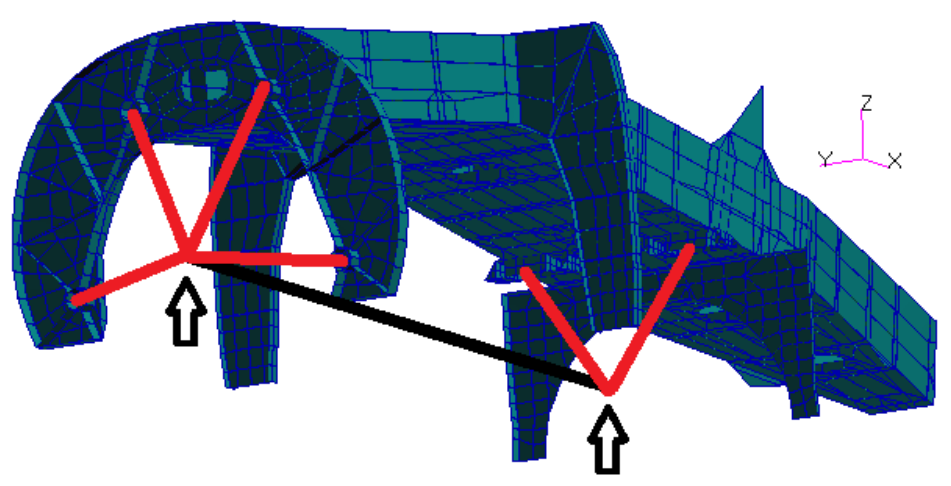

Figura 5.4: Posiciones de los elastómeros en el modelo del capítulo 4 (final líneas rojas) y en este capítulo (flechas)

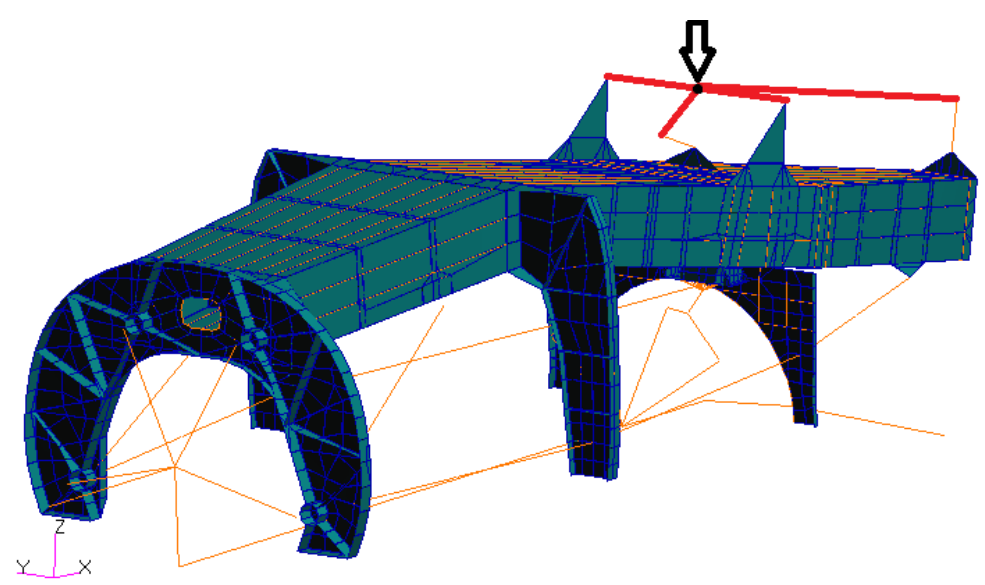

Figura 5.5: Posiciones de los apoyos en el modelo del capítulo (final líneas rojas) 4 y en este capítulo (flecha)

Estos pequeños detalles pueden hacer que en los resultados haya pequeñas diferencias cuantitativas aunque cualitativamente sean similares. En los modelos, por tanto, se pueden 
obtener el mismo tipo de resultado y sirven satisfactoriamente para valorar la utilidad de los mismos con las herramientas de análisis desarrolladas.

\subsubsection{La bancada}

El análisis se realiza con la posibilidad de incluir todo el modelo (modelo E+EMS), como se muestra en la figura 5.3, o sólo el motor (modelo E), como se puede ver en la figura 3.11. El mayor de los inconvenientes del modelo sólo motor es que no es capaz de calcular las fuerzas que específicamente llegan al ala, sólo las que alcanzan la bancada. La incorporación del modelo de la bancada modifica la dinámica del sistema y, por lo tanto, los resultados del análisis.

\subsubsection{Unión motor-bancada}

$\mathrm{Al}$ igual que en el capítulo 4 la unión entre la bancada y el motor se realiza mediante seis apoyos por motor. A modo de recordatorio decir que en los límites admisibles hay diferentes criterios de fallo dependiendo del eje (por un lado la abscisa X y por otro el plano que forma YZ). La forma del criterio de fallo es un elipsoide de revolución. Los tipos de propiedades son los que se muestran en la figura 5.6.

RIGIDEZ APOYOS

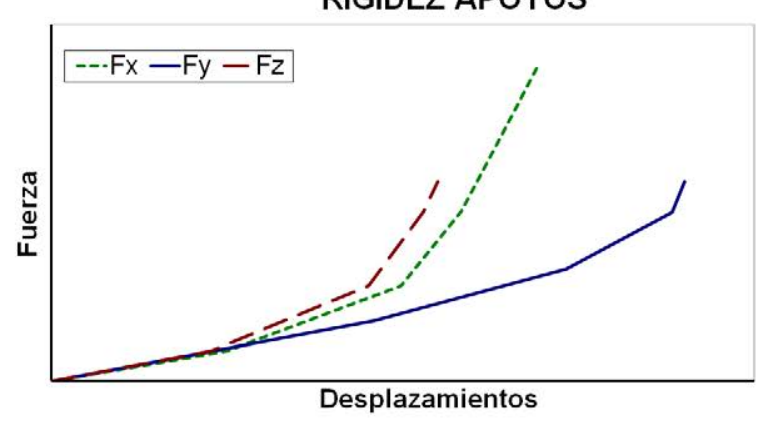

Figura 5.6: Rigidez (izquierda) y resistencia (derecha) de los apoyos

\subsubsection{Unión bancada-ala}

En el modelo la parte delantera de la bancada se apoya en el ala mediante dos puntos (puntos 1 y 2), que soportan fuerzas en X y Z; y la parte trasera se apoya en el ala mediante otros dos puntos (puntos 3 y 4), que soportan fuerzas en $\mathrm{Y}$ y Z respectivamente. El ala se considera infinitamente rígida y fija. Por lo tanto, los admisibles $F_{x 1}$ y $F_{z 1}$ en el punto1, $F_{x 2}$ y $F_{z 2}$ en el punto $2, F_{y 3}$ en el punto 3 y $F_{z 4}$ en el punto 4 se comparan con los resultados de las fuerzas en las condiciones de contorno, ver los límites en la figura 5.7. 

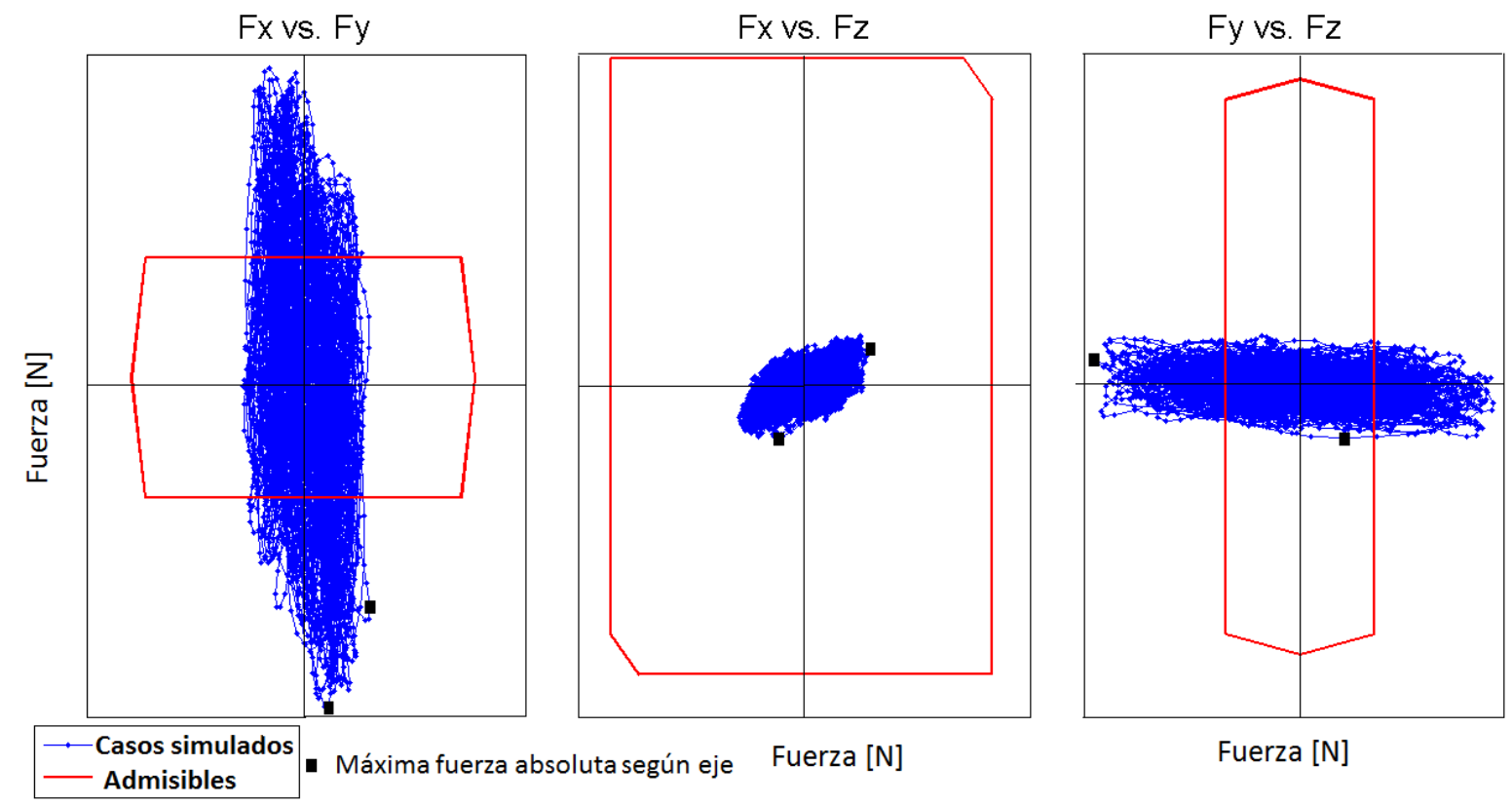

Figura 5.7: Evoluciones de fuerzas X, Y y Z y los valores límites admisibles

\subsubsection{Consideraciones del análisis FEM; método implícito y explícito; paso de tiempo}

Se han llevado a cabo varios chequeos (de masa, inercias, modos propios...). Los primeros modos del modelo FEM son cualitativamente y cuantitativamente coherentes como se ha indicado en 5.2. El comportamiento no-lineal de los dispositivos elastoméricos ha sido reproducido exactamente. Por lo tanto, el motor y su bancada son representados fielmente y el modelo está preparado para simular los análisis no-lineales transitorios. El paso de tiempo crítico para sistemas de múltiples grados de libertad está condicionado por la frecuencia más alta del modo propio que se quiera simular y, por lo tanto, cuanto mayor sea un menor tamaño de incremento de tiempo habrá que utilizar para obtener una solución estable. Como se ha mencionado previamente, si la mayoría de la solución es representada por unos pocos modos de baja frecuencia, es posible utilizar un tamaño de paso de tiempo considerablemente mayor que el límite crítico. Entonces el modelo reduce los modos a tener en cuenta y así se puede incrementar el paso de tiempo como indica la condición de estabilidad, ecuación 2.3.1.2.

Los esquemas de paso de tiempo implícitos e incondicionalmente estables (para problemas lineales) como el método $\beta$-Newmark con $\alpha=0,5$ y $\beta=0,25$ son generalmente los escogidos para resolver este tipo de problemas. Sin embargo, los esquemas implícitos e incondicionalmente estables para problemas lineales suelen comportarse inestablemente al introducirse no-linealidades, especialmente de larga duración en el tiempo. El uso de pasos de tiempo grandes no permite la correcta simulación de los modos propios de frecuencias altas. Estos modos, aunque no contengan la parte importante de la solución, puedes estar contribuyendo a acumular el error para tiempos 
largos, y finalmente afectar a la estabilidad de la solución. Esta es la razón fundamental por la cual se analiza el problema en el capítulo 4 mediante un código explícito, que resuelve los modos propios de alta frecuencia, aunque requiere un gran número de pasos de tiempo. Sin embargo no es el caso una vez realizada la simplificación y optimización y, como se ve a continuación, se utiliza tanto esquemas explícitos como implícitos.

\subsubsection{Esquema de análisis}

Debido a que los dispositivos elastoméricos entre motor y bancada tienen un comportamiento no-lineal (ver apartado 5.2.2.1) la ecuación que rige el fenómeno, por lo tanto, tomará la misma forma que en 2.3 .

Los dispositivos elastoméricos que se están estudiando en la unión del motor son no lineales, de hecho es necesario incluir diferentes comportamientos no lineales de la rigidez en cada eje, como ya se ha visto en 4.4 y se ve en 5.6. Además, estos soportes tienen también diferentes criterios de fallo dependiendo de la dirección. El objetivo es la obtención de curvas elipsoidales como criterios de fallo (eje X como abscisa y YZ como ordenada), cuyos ejes tienen diferentes criterios de fallo, como ya se ha visto en 4.9 y se ve en 5.6.

Como ya sabemos el uso de métodos explícitos requiere resolver la matriz del sistema. La factorización de la matriz del sistema se puede realizar una sola vez si el sistema es lineal, sin embargo, en los casos no lineales se debe realizar muchas veces. Si el paso de tiempo requerido es muy pequeño, el procedimiento se vuelve inabordable y con una duración inaceptable y es necesaria una simplificación profunda. En el caso del método Runge-Kutta, el cálculo del vector de fuerzas internas necesita 4 pasos. Aunque se trata de un proceso menos costoso computacionalmente que resolver la matriz del sistema sigue siendo un coste alto en métodos explícitos, especialmente si intervienen funciones no-lineales.

Los esquemas que se han utilizado se muestran en la tabla 5.2. Todos han sido chequeados y muestran los mismos resultados para varios casos de carga de control.

\begin{tabular}{|c|c|c|}
\hline \multicolumn{3}{|c|}{$y^{\prime}=f(t, y) ; y\left(t_{0}\right)=y_{0}$} \\
\hline $\begin{array}{c}\text { Runge-Kutta } 3^{r d} \text {-order } \\
\text { (Explicit) }\end{array}$ & $\begin{array}{l}y_{n+1}=y_{n}+\frac{h}{9}\left(2 k_{1}+3 k_{2}+4 k_{3}\right) \\
t_{n+1}=t_{n}+h\end{array}$ & $\begin{array}{l}k_{1}=f\left(t_{n}, y_{n}\right) \\
k_{2}=f\left(t_{n}+\frac{h}{2}, y_{n}+\frac{h}{2} k_{1}\right) \\
k_{3}=f\left(t_{n}+\frac{3 h}{4}, y_{n}+\frac{3 h}{4} k_{2}\right)\end{array}$ \\
\hline $\begin{array}{c}\text { Runge-Kutta } 4^{\text {th }} \text {-order } \\
\text { (Explicit) }\end{array}$ & $\begin{array}{l}y_{n+1}=y_{n}+\frac{h}{6}\left(k_{1}+2 k_{2}+2 k_{3}+k_{4}\right) \\
t_{n+1}=t_{n}+h\end{array}$ & $\begin{aligned} k_{1} & =f\left(t_{n}, y_{n}\right) \\
k_{2} & =f\left(t_{n}+\frac{h}{2}, y_{n}+\frac{h}{2} k_{1}\right) \\
k_{3} & =f\left(t_{n}+\frac{h}{2}, y_{n}+\frac{h}{2} k_{2}\right) \\
k_{4} & =f\left(t_{n}+h, y_{n}+h k_{3}\right)\end{aligned}$ \\
\hline $\begin{array}{c}\beta \text {-Newmark } \\
(\alpha=0,5 \text { and } \beta=0,25) \\
\quad(\text { Implicit })\end{array}$ & \multicolumn{2}{|c|}{$\begin{array}{l}y_{n+1}^{\prime}=y_{n}^{\prime}+k\left((1-\alpha) y_{n}^{\prime \prime}+\alpha y_{n+1}^{\prime \prime}\right) \quad 0 \leq \alpha \leq 1 \\
y_{n+1}=y_{n}+h y_{n}^{\prime}+\frac{h^{2}}{2}\left((1-2 \beta) y_{n}^{\prime \prime}+2 \beta y_{n+1}^{\prime \prime}\right) \quad 0 \leq 2 \beta \leq 1 \\
t_{n+1}=t_{n}+h\end{array}$} \\
\hline
\end{tabular}

Tabla 5.2: Esquemas de iteración 


\subsubsection{Niveles de energía}

Como en el capítulo 4 el nivel de energía que se alcanza durante la simulación de la pérdida de pala es un excelente indicador del desarrollo adecuado del fenómeno. La figura 5.8 muestra los distintos tipos de energía que se han chequeado y, en particular, los del caso de carga nominal. En este caso se han considerado tanto la energía cinética, elástica del motor y de la bancada como la energía acumulada en los elastómeros que al romper se pierde y pasa a estar considerada como energía perdida. Como en el capítulo 4 se compara la energía total del sistema con la energía introducida en el sistema siguiendo las mismas ecuaciones que en el apartado 4.4.4.1. La figura 5.9 muestra la energía total y el error cometido en la energía para varios esquemas y pasos de tiempo (5000 y 10000 pasos para 1 segundo de simulación). Se observa que el error en la energía es prácticamente despreciable e insignificante en todos los casos; sin embargo, es diferente entre los distintos esquemas y pasos de tiempo que se utilice. Aunque no sea alto lo que siempre sucede es que siempre se incrementa con el número de pasos. Después de observar las diferencias entre esquemas y debido a su velocidad, estabilidad y versatilidad el esquema que se escoge por defecto para el análisis del resto de casos de carga es el $\beta$-Newmark aunque indistintamente se han cambiado obteniendo los mismos resultados.

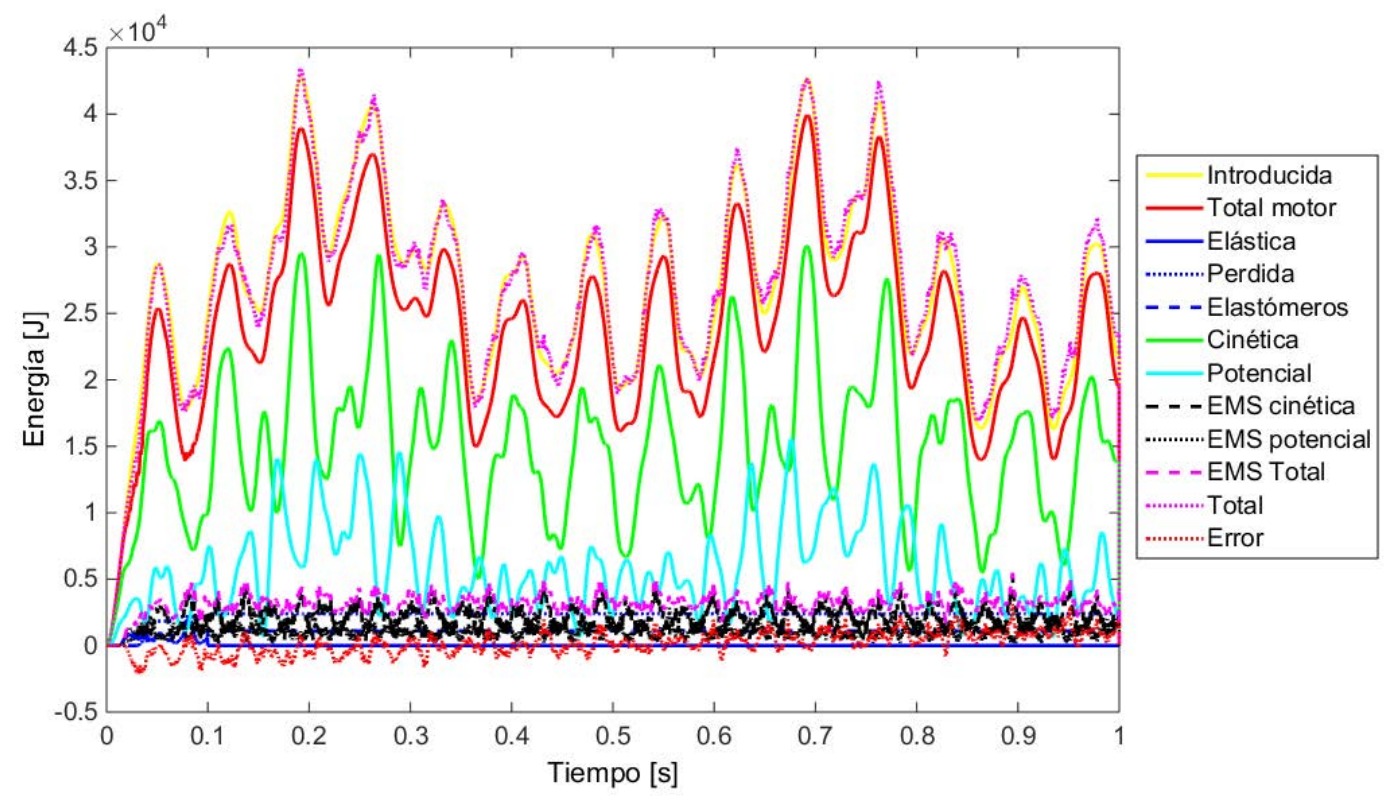

Figura 5.8: Niveles de energía para el caso nominal

El desarrollo de la simulación es muy similar al del capítulo 4, las fuerzas aplicadas debidas a la pérdida de pala rápidamente se convierten en energía gracias al movimiento del punto de aplicación. Casi toda la energía se convierte en energía cinética del motor. Un poco de esa energía cinética, determinada por las propiedades elásticas, alcanza la bancada. Análogamente, otra cantidad de energía pasa a energía interna, más exactamente, energía elástica. La energía 

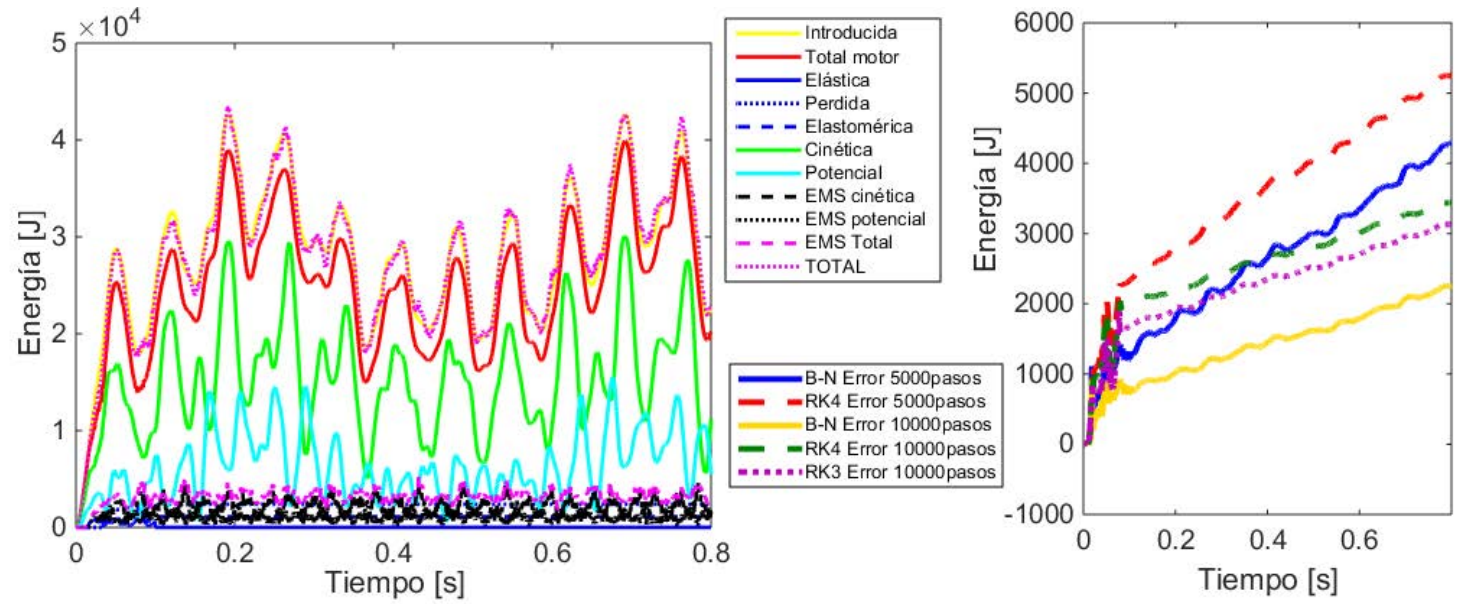

Figura 5.9: Energía total (izquierda) and detalle del error en la energía (derecha) para distintos esquemas y pasos de tiempo

cinética y elástica de la bancada (EMS) influye en el ala a través de las condiciones de contorno. Mientras tanto los elementos del modelo se deforman absorbiendo energía elástica reversible mientras se encuentren en el régimen elástico. Cuando un elemento falla el intercambio de energía deja de ser reversible, y este camino de cargas y energía deja de existir. Estas circunstancias hacen a cada caso de carga diferente, la simulación progresa y cada caso se va separando de los demás. Finalmente, cuando todos los dispositivos elastoméricos fallan el motor se separa de la bancada que queda vibrando a un nivel constante de energía (intercambiando energía cinética y elástica). Este nivel de energía irá disminuyendo suavemente según sea el valor del amortiguamiento estructural aplicado.

\subsubsection{Amortiguamiento estructural}

El amortiguamiento en sistemas mecánicos es la sustracción de energía mecánica del movimiento del sistema, normalmente mediante la conversión de energía potencial en otro tipo de energía que, a la postre, es energía perdida. El aspecto principal a tener en cuenta en este análisis es el teórico efecto positivo que puede tener en el fenómeno, atenuar las oscilaciones. El amortiguamiento estructural se modela mediante un factor de pérdida, que es la energía disipada por radián para la energía potencial máxima en el ciclo. En este caso se ha aplicado sólo para la frecuencia de la hélice que es la única frecuencia de las cargas exteriores aplicadas.

$$
\begin{gathered}
M y^{\prime \prime}+C y^{\prime}+K y=F \\
C=\frac{2 \beta}{\Delta t} M \rightarrow \beta=\eta \omega \Delta t
\end{gathered}
$$

Donde $\beta=$ Parámetro de amortiguamiento estructural, $\eta=$ Coeficiente de amortiguamiento estructural, $\Delta t=$ Incremento de tiempo y $\omega=$ Frecuencia de la hélice. Los valores de $\eta$ se especifican en el apartado 5.3. 


\subsubsection{Cargas}

$\mathrm{Al}$ igual que en el capítulo 4 la pérdida de pala introduce un desequilibrio que se traduce en una fuerza de rotación y un momento en el buje de la hélice (ver figura 3.11). Las fuerzas son debidas principalmente a la fuerza centrípeta que ya no es compensada del trozo de pala que se ha desprendido. Lo mismo pasa con el momento principal que es debido a la fuerza de sustentación del trozo de pala desprendido por el brazo. Ambos dependen de la frecuencia de la hélice. Por lo tanto, las cargas en el punto de aplicación de carga se pueden escribir como; $F_{y}=-F_{c} \cos (\omega t), F_{z}=-F_{c} \sin (\omega t), M_{y}=M_{o o p} \sin (\omega t)$ y $M_{z}=-M_{o o p} \cos (\omega t)$.

\subsection{Casos de carga analizados}

Los casos de carga analizados se obtienen modificando las condiciones iniciales y/o algunos de los parámetros característicos involucrados en el fenómeno de la pérdida de pala. La tabla 5.3 resume los rangos de valores e hipótesis realizadas. Estas hipótesis están basadas en lo explicado en el apartado 2.1.2.1 y se describen a continuación:

- La posición angular de la pala (ANG) cuando se pierde la pala se distribuye de manera uniforme entre $0^{\circ}$ y $360^{\circ}$ debido a que no existe una posición predominante. $0^{\circ}$ ocurre cuando la pala se pierde en dirección paralela al eje Z.

- La velocidad rotacional (RPM) se considera también importante y sus valores se encuentran uniformemente distribuidos entre dos típicas condiciones de vuelo con mínima y máxima velocidad (660rpm and 840rpm).

- Otro parámetro es el tamaño de pala de perdida (SIZE) ya que se puede perder totalmente la pala $($ ratio $=0)$ o parcialmente $($ ratio $>0)$.

- La rigidez de los elastómeros (STIFF) y su resistencia (STR) se distribuyen de forma normal alrededor de sus valores nominales. En ambos casos, sus valores se encuentran en un $\pm 10 \%$ del valor nominal en el $68 \%$ de los casos, en un $\pm 20 \%$ del valor nominal en el $95 \%$ de los casos y en un $\pm 30 \%$ del valor nominal en el $99,7 \%$ de los casos.

- Finalmente, el amortiguamiento estructural (DAMP) que se considera distribuido uniformemente entre $0 \%$ y $5 \%$ (valores característicos de materiales estructurales).

\subsubsection{Técnica del método de Monte Carlo (MCT)}

La técnica MCT permite estudiar el problema planteado con las variables aleatorias que se han descrito, la manera de realizarlo se detalla en la referencia JCGM (2008). Las variables de entrada en MCT son las funciones de distribución y el número de simulaciones del fenómeno $(\mathrm{N})$. 


\begin{tabular}{|c|c|c|c|}
\hline Variable & Distribución & Intervalo & Valor Nominal \\
\hline Posición angular de pala perdida (ANG) & Uniforme & $\left(0^{\circ}, 360^{\circ}\right)$ & $0^{\circ}$ \\
\hline Sensibilidad a las rpm (RPM) & Uniforme & $(69,88)$ & $\omega_{\text {nominal }}$ \\
\hline Tamaño de pala perdida (SIZE) & Uniforme & $(0,1)$ & 1 \\
\hline Rigidez de los elastómeros (STIFF) & Normal & $\mathrm{N}(0,1)$ & 1 \\
\hline Resistencia de los elastómeros (STR) & Normal & $\mathrm{N}(0,1)$ & 1 \\
\hline Amortiguamiento estructural (DAMP) & Uniforme & $(0 \%, 5 \%)$ & 0 \\
\hline
\end{tabular}

Tabla 5.3: Propiedades de las variables de entrada

En una primera fase las funciones de distribución se generan en Matlab siguiendo las funciones definidas en la tabla 5.3. No hay correlaciones entre ellas ya que se han identificado exactamente las funciones inicialmente necesarias e independientes, las demás son derivadas de las primeras, como por ejemplo la amplitud de la fuerza de la carga por la pérdida de pala que es derivada del tamaño de pala perdida, lo mismo sucede con los momentos de la carga. El número de simulaciones se ha seleccionado entre 1000 y 10000 a lo largo de todo el estudio.

En la siguiente fase la ecuación motor $\left(Y_{\text {OUTPUTs }}=f\left(X_{\text {INPUTs }}\right)\right)$ se simula $\mathrm{N}$ veces, realizándose $\mathrm{N}$ evaluaciones. Se integra la ecuación 2.3 en el dominio del tiempo por medio de métodos de integración que se han explicado en el apartado 5.2.3.2 y descrito en el apartado B.2. Finalmente, las distribuciones de salida son analizadas y comparadas.

\subsubsection{Configuración de los parámetros de entrada}

La técnica MCT permite seleccionar diferentes configuraciones de parámetros de entrada con el fin de obtener una imagen más clara de sus correlaciones e interacciones. En el estudio se ha considerado la variación de un sólo parámetro, ANG, RPM, SIZE, STIFF, STR o DAMP, ver figura 5.10 donde una línea negra un poco más gruesa separa los datos de entrada con los de salida, la variación simultánea de dos parámetros, ver figura 5.11 donde líneas negras un poco más gruesas separan los datos de entrada con los de salida. Por razones de influencia de los parámetros sólo se han post-procesado, en el caso de dos parámetros, otro parámetro además del tamaño de pala perdida, por lo tanto, SIZE\&ANG, SIZE\&RPM, SIZE\&STIFF, SIZE\&STR o SIZE\&DAMP. Finalmente la variación simultánea de todos los parámetros de entrada, ALL o TODO, ver figura 5.12 donde también se separa con una línea más gruesa.

La simulación puede tener en cuenta sólo el motor o el motor y la bancada, como se ha explicado en el apartado 5.2.2. Cabe destacar que debido al desarrollo realizado, cronológicamente se dispuso inicialmente del modelo (E) con todas las variables de entrada salvo DAMP y, por lo tanto, esos fueron los primeros resultados preliminares que se obtuvieron como se ve en el apartado 5.4.1. Más adelante se pudo incluir tanto la variable DAMP como el modelo completo (E+EMS).

Estas configuraciones diferentes de entrada permiten obtener resultados muy útiles e interesantes que no se conseguirían con otros métodos. En los siguientes apartados se muestran estos resultados. 


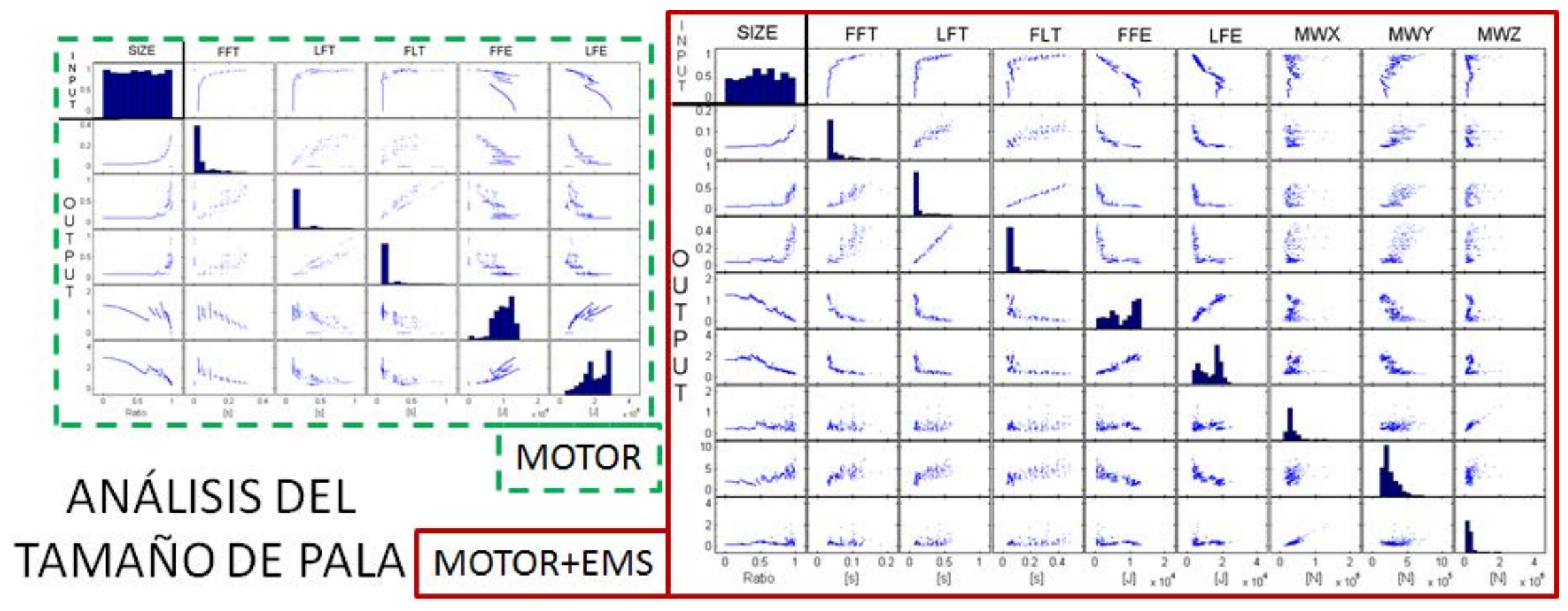

Figura 5.10: Resultados de la pérdida de pala para el modelo sólo motor (izquierda) e incluyendo la bancada (derecha) 


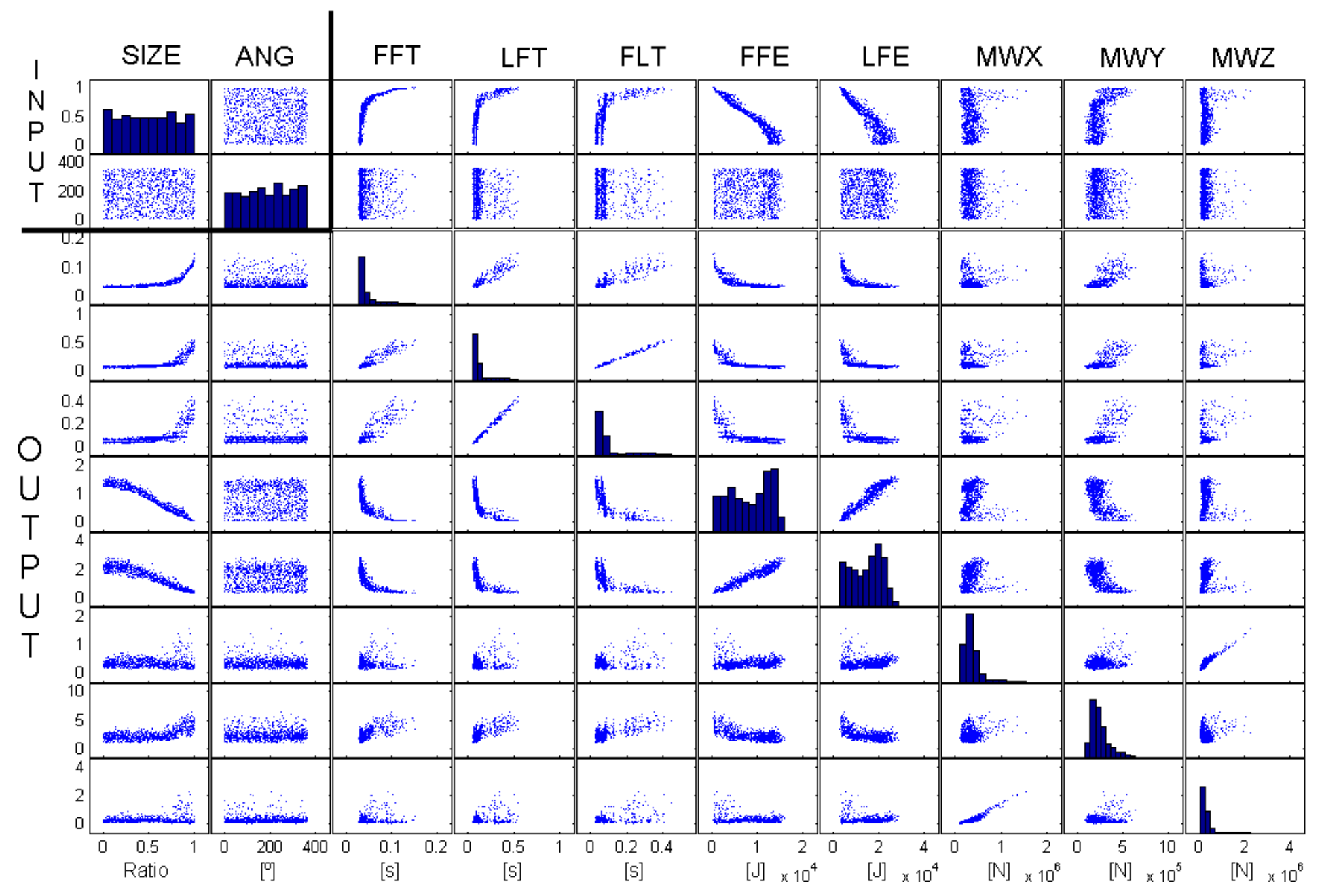

Figura 5.11: Resultados de tamaño de pala perdida y posición angular 


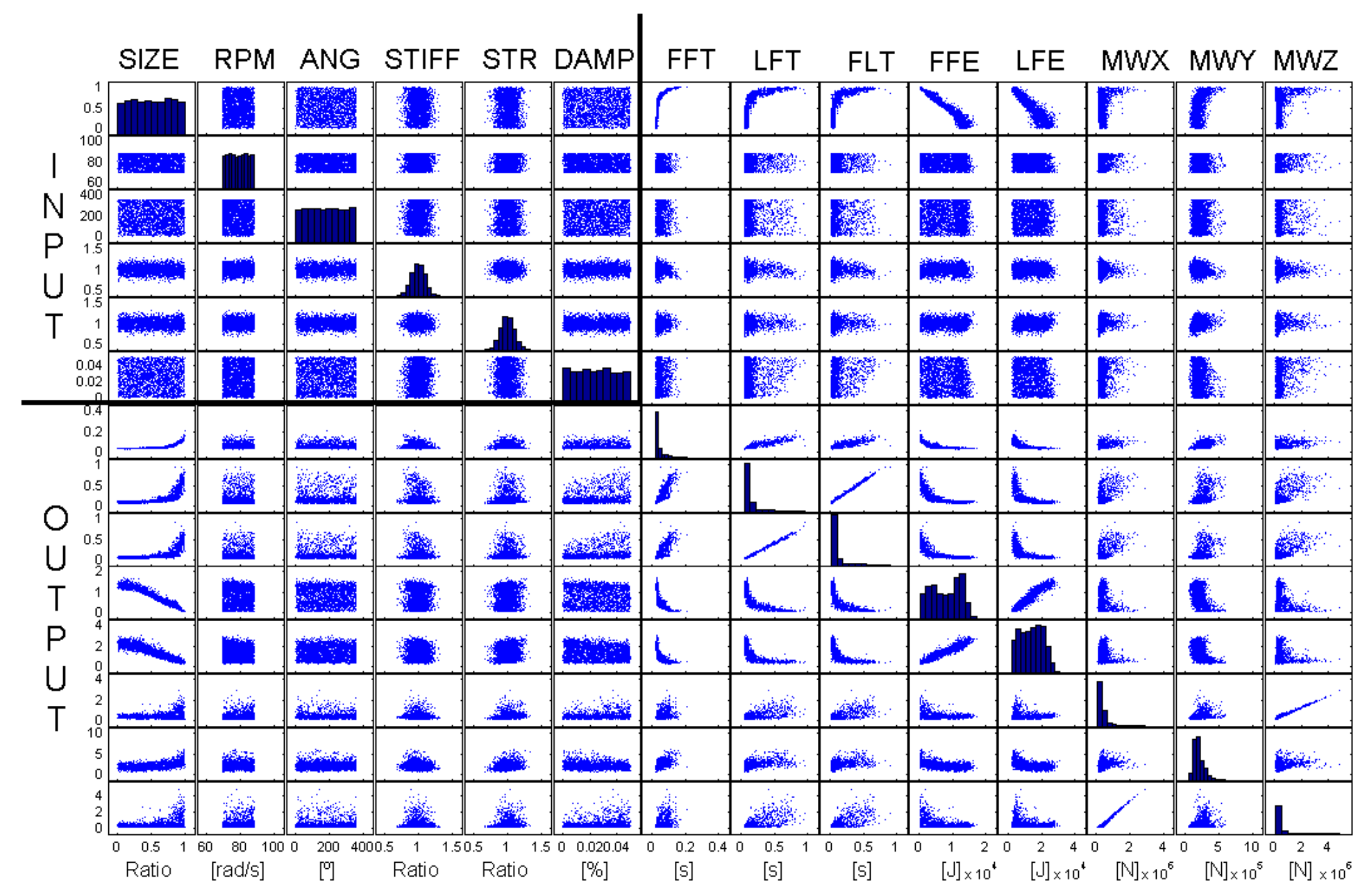

Figura 5.12: Resultados con variación de todos los parámetros de entrada 


\subsection{Resultados}

Ente las variables de salida se han elegido analizar dos valores de tiempo de fallo: el tiempo de la primera rotura (FFT), que es el tiempo en el que se produce la rotura del primer elastómero, y el tiempo de la última rotura (LFT), que es el tiempo en el que se rompe el último elastómero y, en consecuencia, el motor queda completamente separado de la estructura de la aeronave. La diferencia entre estos dos tiempos (FLT a partir de ahora) proporciona el tiempo total entre el principio de la secuencia de fallo hasta la completa separación del motor. Además, se han considerado las variables siguientes: la energía cuando se produce la primera rotura (FFE), la energía cuando se produce la última rotura (LFE) y las máximas fuerzas que alcanzan el ala en los diferentes ejes X, Y y Z a lo largo de toda la simulación (MWX, MWY \& MWZ). Existen otras variables que se han utilizado como son: fuerzas elásticas en los muelles, secuencia de fallo, ángulo de fallo... La tabla 5.4 muestra las medias y desviaciones típicas de las variables más importantes cuando se varía lo que se indica en la primera columna (los parámetros de entrada se han explicado en el apartado 5.3 y el tipo de variación se indica en la tabla 5.3) mientras que los demás parámetros se mantienen constantes en su valor nominal. Asimismo, otra variable que ha sido post-procesada a partir de la variable de entrada RPM y de la variable de salida FFT es el ángulo de la pala dañada en el plano de rotación de la hélice en el que se produce la primera rotura elastomérica, PAF. Análogamente, la variable PAL se puede post-procesar a partir de RPM y LFT e indica el ángulo de la pala dañada en el plano de rotación de la hélice en el que se produce la última rotura de elastómero. PAF \& PAL tienen el mismo origen que la variable ANG. Estas dos variables se muestran en la tabla 5.4 donde se indican el intervalo de los ángulos más propensos en los que se producen roturas de los apoyos elastoméricos. (E) indica que el análisis sólo ha considerado el motor y (E+EMS) que ha considerado también la bancada, como se ha explicado en el apartado 5.2.2.

\subsubsection{Resultados preliminares de variaciones individuales con el modelo sólo motor}

Como ya se ha mencionado inicialmente se dispuso del modelo (E) sin incluir la bancada, y sólo se variaron el ángulo en el que se produce la pérdida de la pala, la sensibilidad a las rpm, el tamaño de la pala perdida, la rigidez de los elastómeros, y la resistencia de los elastómeros (no se incluyó el amortiguamiento). El problema se analiza mediante la técnica de Monte Carlo realizando una batería de simulaciones. Los parámetros del modelo son como los mostrados en la tabla 5.3 aunque sin la variación del amortiguamiento estructural.

Estos análisis preliminares muestran que el impacto en los resultados de las variaciones de los parámetros es diferente, la matriz de correlación entre todos los parámetros se puede ver en la figura 5.13.

Se observa que el parámetro más influyente es el tamaño de pérdida de pala. 


\begin{tabular}{|c|c|c|c|c|c|c|c|c|c|c|c|c|}
\hline \multicolumn{2}{|l|}{$\begin{array}{c}\text { CASOS DE } \\
\text { CARGA }\end{array}$} & $\begin{array}{l}\text { FFT } \\
{[\mathrm{s}]}\end{array}$ & $\begin{array}{c}\text { LFT } \\
{[\mathrm{s}]}\end{array}$ & $\begin{array}{c}\text { FLT } \\
{[\mathrm{s}]}\end{array}$ & $\begin{array}{c}\text { FFE } \\
{[\mathrm{J}]}\end{array}$ & $\begin{array}{c}\mathrm{LFE} \\
{[\mathrm{J}]}\end{array}$ & $\begin{array}{c}\text { MWX } \\
{[\mathrm{N}]}\end{array}$ & $\begin{array}{c}\text { MWY } \\
{[\mathrm{N}]}\end{array}$ & $\begin{array}{c}\text { MWZ } \\
{[\mathrm{N}]}\end{array}$ & & $\begin{array}{c}\text { PAF } \\
{\left[{ }^{\circ}\right]}\end{array}$ & $\begin{array}{l}\text { PAL } \\
\left.{ }^{\circ}\right]\end{array}$ \\
\hline \multirow{2}{*}{$\begin{array}{c}A N G \\
(E+E M S)\end{array}$} & $\mu$ & 0,0306 & 0,0757 & 0,0450 & 13712 & 21660 & 340466 & 201073 & 257747 & $\theta_{i n i}$ & 170 & 150 \\
\hline & $\sigma$ & 0,0015 & 0,0129 & 0,0128 & 922 & 2677 & 89630 & 49981 & 86664 & $\theta_{\text {fin }}$ & 330 & 170 \\
\hline \multirow{2}{*}{$\begin{array}{c}R P M \\
(E+E M S)\end{array}$} & $\mu$ & 0,0278 & 0,0669 & 0,0391 & 13141 & 21503 & 223687 & 197536 & 143490 & $\theta_{i n i}$ & 340 & 0 \\
\hline & $\sigma$ & 0,0001 & 0,0013 & 0,0012 & 252 & 2138 & 5383 & 6205 & 9767 & $\theta_{\text {fin }}$ & 170 & 360 \\
\hline \multirow{2}{*}{$\begin{array}{c}S I Z E \\
(E+E M S)\end{array}$} & $\mu$ & 0,0442 & 0,1266 & 0,0824 & 7930 & 13119 & 313102 & 243175 & 253614 & $\theta_{i n i}$ & 170 & 0 \\
\hline & $\sigma$ & 0,0228 & 0,1000 & 0,0797 & 3915 & 5582 & 109562 & 101409 & 154733 & $\theta_{\text {fin }}$ & 180 & 10 \\
\hline \multirow{2}{*}{$\begin{array}{c}\text { STIFF } \\
(E+E M S)\end{array}$} & $\mu$ & 0,0281 & 0,0658 & 0,0377 & 12823 & 18765 & 263458 & 208001 & 147308 & $\theta_{i n i}$ & 160 & 180 \\
\hline & $\sigma$ & 0,0007 & 0,0051 & 0,0050 & 218 & 1646 & 43411 & 27714 & 40731 & $\theta_{\text {fin }}$ & 170 & 190 \\
\hline \multirow{2}{*}{$\begin{array}{c}S T R \\
(E+E M S)\end{array}$} & $\mu$ & 0,0275 & 0,0742 & 0,0467 & 12652 & 19697 & 255455 & 200416 & 182289 & $\theta_{i n i}$ & 170 & $0 / 160$ \\
\hline & $\sigma$ & 0,0008 & 0,0165 & 0,0162 & 316 & 3375 & 52226 & 26546 & 92090 & $\theta_{\text {fin }}$ & 170 & $50 / 200$ \\
\hline \multirow{2}{*}{$\begin{array}{c}D A M P \\
(E+E M S)\end{array}$} & $\mu$ & 0,0286 & 0,0945 & 0,0659 & 11810 & 19175 & 224477 & 168536 & 236314 & $\theta_{i n i}$ & 170 & 10 \\
\hline & $\sigma$ & 0,0010 & 0,0146 & 0,0142 & 661 & 1860 & 33505 & 11052 & 69636 & $\theta_{\text {fin }}$ & 240 & 50 \\
\hline \multirow{2}{*}{$\begin{array}{c}S I Z E \& A N G \\
(E+E M S)(*)\end{array}$} & $\mu$ & 0,0443 & 0,1317 & 0,0874 & 8428 & 14706 & 357928 & 242325 & 313238 & $\theta_{i n i}$ & 0 & 0 \\
\hline & $\sigma$ & 0,0218 & 0,0946 & 0,0749 & 4404 & 6464 & 161059 & 92356 & 241025 & $\theta_{\text {fin }}$ & 360 & 360 \\
\hline \multirow{2}{*}{$\begin{array}{c}S I Z E \& R P M \\
(E+E M S)(*)\end{array}$} & $\mu$ & 0,0440 & 0,1220 & 0,0780 & 8209 & 14510 & 289338 & 235145 & 233283 & $\theta_{i n i}$ & 0 & 0 \\
\hline & $\sigma$ & 0,0234 & 0,0899 & 0,0692 & 4096 & 6561 & 128034 & 99520 & 194100 & $\theta_{\text {fin }}$ & 360 & 360 \\
\hline \multirow{2}{*}{$\begin{array}{c}S I Z E \& S T I F F \\
(E+E M S)\end{array}$} & $\mu$ & 0,0447 & 0,1260 & 0,0813 & 8136 & 13639 & 314920 & 244503 & 249958 & $\theta_{i n i}$ & 170 & 350 \\
\hline & $\sigma$ & 0,0244 & 0,0989 & 0,0766 & 4071 & 5820 & 120768 & 100411 & 163437 & $\theta_{\text {fin }}$ & 180 & 30 \\
\hline \multirow{2}{*}{$\begin{array}{c}S I Z E \& S T R \\
(E+E M S)\end{array}$} & $\mu$ & 0,0445 & 0,1248 & 0,0802 & 7989 & 13721 & 328886 & 252132 & 265176 & $\theta_{i n i}$ & 160 & 350 \\
\hline & $\sigma$ & 0,0234 & 0,0934 & 0,0730 & 3983 & 6414 & 126674 & 100045 & 172826 & $\theta_{\text {fin }}$ & 170 & 10 \\
\hline \multirow{2}{*}{$\begin{array}{c}\text { SIZE\&DAMP } \\
(E+E M S)\end{array}$} & $\mu$ & 0,0462 & 0,1713 & 0,1251 & 7615 & 12792 & 338488 & 202738 & 400658 & $\theta_{i n i}$ & 0 & 0 \\
\hline & $\sigma$ & 0,0249 & 0,1657 & 0,1439 & 3811 & 5883 & 260376 & 80312 & 471957 & $\theta_{\text {fin }}$ & 360 & 30 \\
\hline \multirow{2}{*}{$\begin{array}{c}A L L \\
(E+E M S)(*)\end{array}$} & $\mu$ & 0,0458 & 0,1600 & 0,1142 & 8130 & 14053 & 364870 & 198296 & 414076 & $\theta_{i n i}$ & 0 & 0 \\
\hline & $\sigma$ & 0,0230 & 0,1406 & 0,1203 & 4266 & 6416 & 270579 & 76536 & 478869 & $\theta_{\text {fin }}$ & 360 & 360 \\
\hline \multirow{2}{*}{$\begin{array}{c}A N G \\
(E)\end{array}$} & $\mu$ & 0,0179 & 0,0890 & 0,0711 & 9376 & 21619 & & & & $\theta_{i n i}$ & 170 & 0 \\
\hline & $\sigma$ & 0,0018 & 0,0084 & 0,0075 & 1364 & 4450 & & & & $\theta_{\text {fin }}$ & 200 & 360 \\
\hline \multirow{2}{*}{$\begin{array}{c}R P M \\
(E)\end{array}$} & $\mu$ & 0,0236 & 0,0921 & 0,0684 & 14007 & 30346 & & & & $\theta_{i n i}$ & 250 & 0 \\
\hline & $\sigma$ & 0,0003 & 0,0072 & 0,0074 & 431 & 3326 & & & & $\theta_{\text {fin }}$ & 10 & 360 \\
\hline \multirow{2}{*}{$\begin{array}{l}S I Z E \\
(E)(*)\end{array}$} & $\mu$ & 0,0455 & 0,1533 & 0,1078 & 9916 & 20312 & & & & $\theta_{i n i}$ & 0 & 340 \\
\hline & $\sigma$ & 0,0467 & 0,1236 & 0,0893 & 2732 & 6894 & & & & $\theta_{\text {fin }}$ & 30 & 10 \\
\hline \multirow{2}{*}{$\begin{array}{c}\text { STIFF } \\
(E)\end{array}$} & $\mu$ & 0,0230 & 0,1013 & 0,0782 & 13217 & 29460 & & & & $\theta_{i n i}$ & 0 & 350 \\
\hline & $\sigma$ & 0,0002 & 0,0022 & 0,0022 & 65 & 1172 & & & & $\theta_{\text {fin }}$ & 30 & 360 \\
\hline \multirow{2}{*}{$\begin{array}{c}S T R \\
(E) \\
\end{array}$} & $\mu$ & 0,0217 & 0,0968 & 0,0751 & 12268 & 26141 & & & & $\theta_{i n i}$ & 0 & 350 \\
\hline & $\sigma$ & 0,0027 & 0,0086 & 0,0061 & 1949 & 6259 & & & & $\theta_{\text {fin }}$ & 20 & 10 \\
\hline$D A M P$ & $\mu$ & 0,0233 & 0,1027 & 0,0795 & 12586 & 22497 & & & & $\theta_{i n i}$ & 0 & 350 \\
\hline$(E)$ & $\sigma$ & 0,0001 & 0,0006 & 0,0005 & 387 & 3643 & & & & $\theta_{\text {fin }}$ & 30 & 50 \\
\hline$S I Z E \& A N G$ & $\mu$ & 0,0454 & 0,1469 & 0,1015 & 9166 & 17646 & & & & $\theta_{i n i}$ & 160 & 0 \\
\hline$(E)$ & $\sigma$ & 0,0514 & 0,1248 & 0,0823 & 2810 & 5107 & & & & $\theta_{\text {fin }}$ & 290 & 360 \\
\hline$S I Z E \& R P M$ & $\mu$ & 0,0447 & 0,1484 & 0,1037 & 10343 & 22299 & & & & $\theta_{i n i}$ & 260 & 0 \\
\hline$(E)$ & $\sigma$ & 0,0440 & 0,1269 & 0,0989 & 3076 & 7009 & & & & $\theta_{\text {fin }}$ & 80 & 360 \\
\hline$S I Z E \& S T I F F$ & $\mu$ & 0,0437 & 0,1533 & 0,1095 & 9862 & 20250 & & & & $\theta_{i n i}$ & 0 & 350 \\
\hline$(E)$ & $\sigma$ & 0,0447 & 0,1283 & 0,0961 & 2861 & 6778 & & & & $\theta_{\text {fin }}$ & 80 & 10 \\
\hline$S I Z E \& S T R$ & $\mu$ & 0,0446 & 0,1487 & 0,1041 & 9625 & 19518 & & & & $\theta_{i n i}$ & 0 & 350 \\
\hline$(E)$ & $\sigma$ & 0,0466 & 0,1159 & 0,0775 & 2649 & 6584 & & & & $\theta_{\text {fin }}$ & 100 & 90 \\
\hline$S I Z E \& D A M P$ & $\mu$ & 0,0326 & 0,1548 & 0,1222 & 8469 & 16136 & & & & $\theta_{i n i}$ & 10 & 350 \\
\hline$(E)$ & $\sigma$ & 0,0324 & 0,1529 & 0,1340 & 3979 & 5427 & & & & $\theta_{\text {fin }}$ & 40 & 70 \\
\hline$A L L$ & $\mu$ & 0,0344 & 0,1533 & 0,1189 & 8345 & 16719 & & & & $\theta_{i n i}$ & 0 & 0 \\
\hline$(E)$ & $\sigma$ & 0,0350 & 0,1338 & 0,1176 & 3830 & 5840 & & & & $\theta_{\text {fin }}$ & 360 & 360 \\
\hline $\begin{array}{c}\mu=\text { Media }, \\
(*)=(\text {SIZE\&A }\end{array}$ & & iació & pica, $\theta$ & $-\theta_{f i}$ & Int & lo ar & 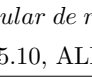 & & & & $=$ & cada \\
\hline
\end{tabular}

Tabla 5.4: Propiedades de los parámetros de salida 


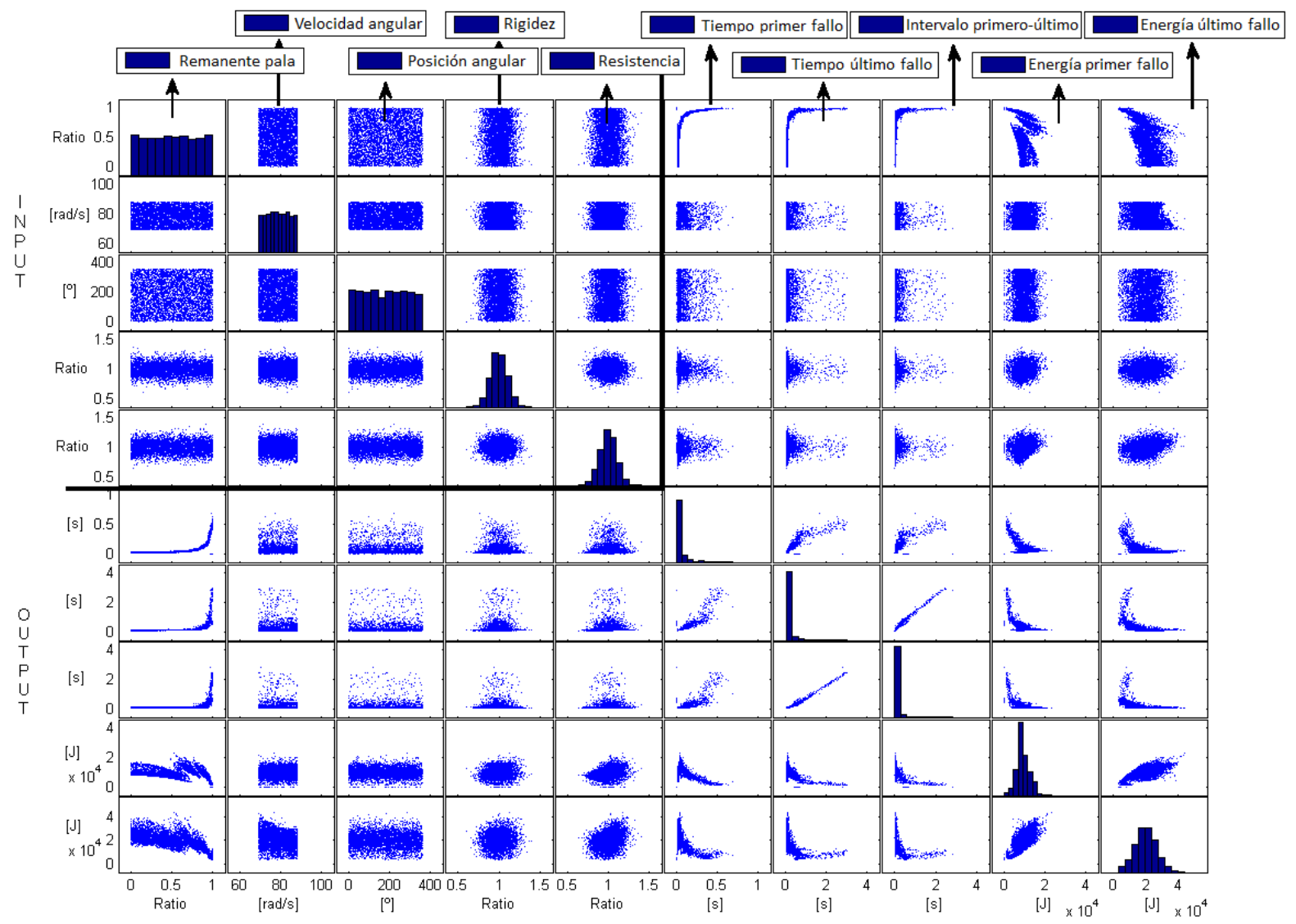

Figura 5.13: Matriz de correlación con varias variables de entrada y de salida de la simulación

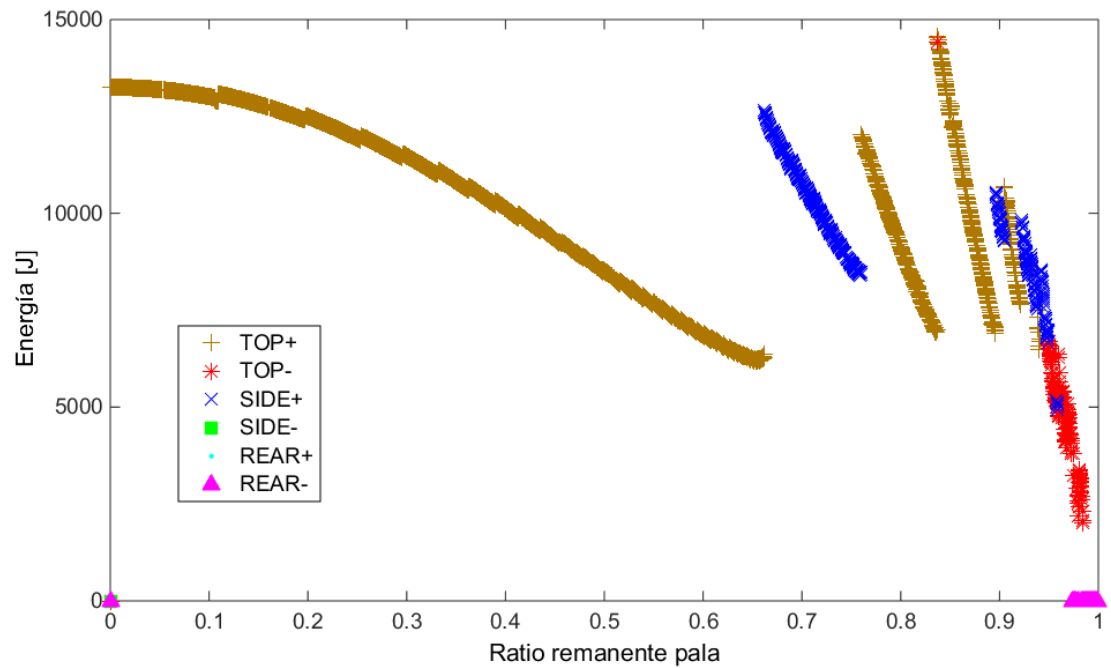

Figura 5.14: Energía del modelo en el momento de la primera rotura vs. el ratio de pala no desprendida 
Como se muestra la técnica de Monte Carlo permite estudiar el problema con diferentes variaciones de parámetros. Esto permite que las variaciones de cada parámetro se puedan clasificar en relación con la variabilidad que produce en los resultados, ver la figura 5.15. La figura muestra como hay mayor dispersión en los resultados cuando se varía la frecuencia del motor a la que sucede la pérdida o la posición angular en que se encuentra la pala perdida cuando se separa, además del tamaño de pérdida de pala.

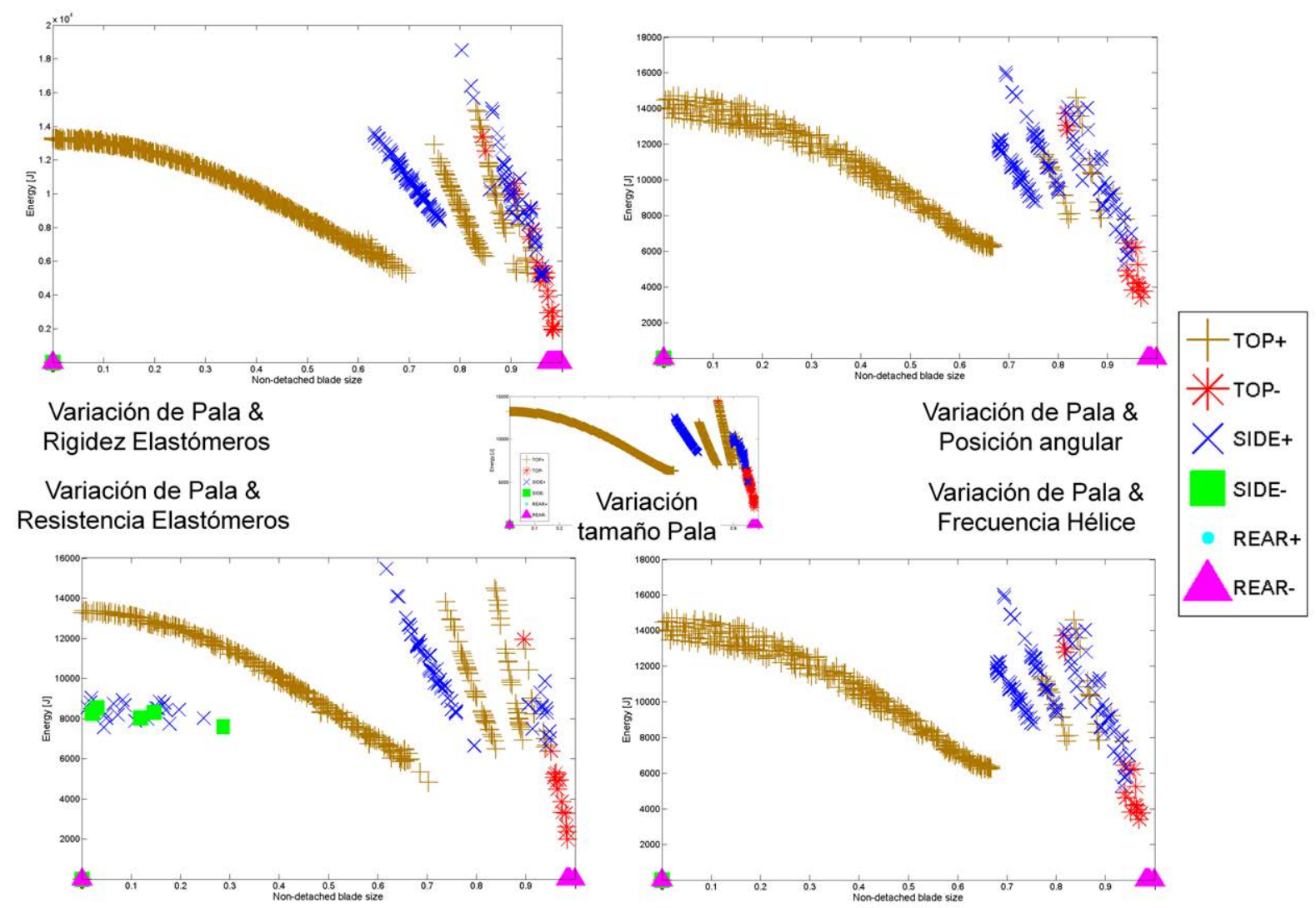

Figura 5.15: Distintas variaciones de parámetros vs. el ratio de pala no desprendida

Por lo tanto, la evolución de los resultados del análisis cuando se cambia el tamaño de la pala perdida es mucho mayor a cuando se cambia la rigidez de los elastómeros o la resistencia de los elastómeros. En un rango medio de influencia se encuentra la frecuencia del motor a la que sucede la pérdida y la posición angular en que se encuentra la pala perdida cuando se separa.

Los resultados preliminares con variaciones individuales de parámetros que se dispusieron fueron:

- Ángulo en el que se produce la pérdida de la pala (ANG): Los resultados no muestran una alta dependencia de este parámetro. La primera rotura de elastómero se produce en $\mathrm{t}=$ $0,018 \mathrm{~s}$ (en media) y sobre todo entre $\theta=170^{\circ}-200^{\circ}$ y el último se distribuye a lo largo del ángulo $\theta$. La secuencia de rotura cambia con el valor de este parámetro. 
- Sensibilidad a las rpm (RPM): Los resultados no muestran una alta dependencia de este parámetro. La primera rotura de elastómero se produce en $\mathrm{t}=0,024 \mathrm{~s}$ (en media) y sobre todo entre $\theta=260^{\circ}-350^{\circ}$ y el último se distribuye a lo largo del ángulo $\theta$.

- Tamaño de la pala perdida (SIZE): El tiempo necesario para separar el motor de la aeronave es mayor cuando la pala perdida es más pequeña. Además, el nivel de energía del modelo cuando falla el primer elastómero sigue unos patrones claros y definidos. La primera rotura se produce en $\mathrm{t}=0,046 \mathrm{~s}$ (en media) y principalmente entre $\theta=0^{\circ}-50^{\circ}$, la última rotura ocurre normalmente entre $\theta=350^{\circ}-10^{\circ}$. En este parámetro existen comportamientos muy dispares dependiendo del valor del parámetro, los intervalos son: 0-0,66, 0,66-0,76, $0,76-0,83,0,83-0,9, \ldots$ (figura 5.14 ).

- Rigidez de los elastómeros (STIFF): Los resultados no muestran una alta dependencia de este parámetro. La primera rotura de elastómero se produce principalmente en $\mathrm{t}=0,023$ s (en media) y entre $\theta=0^{\circ}-10^{\circ}$, el último normalmente ocurre entre $\theta=350^{\circ}-0^{\circ}$.

- Resistencia de los elastómeros (STR): Los resultados mostraron dos comportamientos disímiles con fallo distinto y a diferentes niveles de energía. La primera rotura de elastómero se produce en $\mathrm{t}=0,022 \mathrm{~s}$ (en media) y principalmente entre $\theta=10^{\circ}-30^{\circ}$ ó $\theta=150^{\circ}-200^{\circ}$, el último normalmente ocurre entre $\theta=350^{\circ}-10^{\circ}$ ó $\theta=50^{\circ}-90^{\circ}$, respectivamente.

\subsubsection{Resultados de variaciones individuales de los parámetros de entrada}

A continuación, se evalúan los resultados de la variaciones de un sólo parámetro de entrada para el modelo completo, E+EMS y una vez implementada también la opción de variar el amortiguamiento. Los resultados obtenidos fueron:

- Posición angular de la pala perdida (ANG) (ver figura 5.20). El primer elastómero rompe, de media, en $t=0,031 \mathrm{~s}$. El último ocurre, de media, en $t=0,076 \mathrm{~s}$. Prácticamente no hay diferencias de resultados entre las diferentes simulaciones. Existen dos familias de resultados en lo que respecta a la variable FLT (una alrededor de $t=0,065 \mathrm{~s}$ y la otra alrededor de $t=0,095 \mathrm{~s}$ ), sin embargo, la distinción entre las dos familias no depende de ANG, como se puede observar en la figura 5.16.

- Sensibilidad a las rpm (RMP) (ver figura 5.19). Los resultados no muestran una gran dependencia de este parámetro. Sin embargo, los resultados no son como a priori cabría esperar. El primer elastómero rompe, de media, en $t=0,028 \mathrm{~s}$. El último ocurre, de media, en $t=0,067 \mathrm{~s}$. La variable MWZ decrece cuando se incrementa RPM, como se puede ver en la figura 5.16, y es exactamente lo contrario que sucede con la fuerza en X. La variable FFE decrece cuando RPM crece. Finalmente, los ángulos más probables en los que se produzca una rotura PAF y PAL se muestran en la figura 5.17, PAF está principalmente 
en el intervalo $\theta=290^{\circ}-10^{\circ}$, y PAL se encuentra a lo largo de todo el rango de $\theta$. Este resultado también viene recogido en la última columna de la tabla 5.4.

- Tamaño de pala perdida (SIZE) (ver figura 5.18). El tiempo necesario para separar el motor de la aeronave es mayor cuando la pala perdida es menor. Además, el nivel de la energía de modelo cuando se rompe el primer elastómero sigue patrones específicos de comportamiento. El primer elastómero rompe, de media, en $t=0,044 s$ y el último normalmente ocurre, de media, en $t=0,127 \mathrm{~s}$, hay considerables diferencias de resultados según se varía SIZE. Según sea el parámetro las simulaciones evoluciones de distinta manera, los intervalos de valores del parámetro son $0-0,67,0,67-0,78,0,78-0,85,0,85-0,9$, un ejemplo se muestra en la figura 5.16. Éste es el motivo por el cual la desviación típica es muy alta (por ejemplo 0.023s para FFT) comparado con otras variaciones de parámetros de entrada. FFE se incrementa cuando SIZE decrece (ver la figura 5.16). En el caso de MWY, su valor decrece cuando también lo hace el de SIZE (ver la figura 5.16), sin embargo las fuerzas MWX \& MWZ permanecen aproximadamente constantes. La mayoría de los resultados se pueden ver en la figura 5.10. Finalmente, los ángulos más probables en los que se produzca una rotura PAF y PAL se muestran en la figura 5.17, PAF vale siempre $\theta=170^{\circ}$, y PAL $\theta=10^{\circ}$ cuando el valor de SIZE es bajo. Debido a que RPM es constante (valor nominal) la función PAF and PAL son equivalentes a las variables FFT y FLT.

- Rigidez de los elastómeros (STIFF) (ver figura 5.21). Los resultados no muestran una gran dependencia de este parámetro, ver la figura 5.16. La rotura del primer elastómero suele ocurrir, de media, en $t=0,028 \mathrm{~s}$, la última normalmente sucede, de media, en $t=0,066 \mathrm{~s}$. Los valores de la energía y máximas fuerzas en los distintos ejes varían con este parámetro, sin embargo, no están correlados.

- Rigidez de los elastómeros (STR) (ver figura 5.22). Los resultados muestran dos comportamientos distintos con distintas secuencias de fallo a diferentes niveles de energía; uno se produce cuando el valor del parámetro se encuentra en el intervalo 0,7-0,89 y el otro en el intervalo 0,89-1,3 (ver la figura 5.16). El primer elastómero rompe, de media, en $t=0,0275 \mathrm{~s}$ (para bajos valores de rigidez en $0.028 \mathrm{~s}$ y para altos en $0.025 \mathrm{~s}$ ) y PAF es aproximadamente $\theta=170^{\circ}$. La última rotura normalmente ocurre, de media, en $t=0,074 s$ y PAL ocurre normalmente en el intervalo $\theta=0^{\circ}-50^{\circ}$ (para bajas rigideces) o $\theta=160^{\circ}-200^{\circ}$ (para altas rigideces). Además, las fuerzas máximas que llegan al ala decrecen cuando lo hace STR (ver la figura 5.16). Finalmente destacar que FFE decrece cuando también lo hace STR.

- Amortiguamiento estructural (DAMP) (ver figura 5.23). El primer elastómero rompe, de media, en $t=0,029 \mathrm{~s}$ y el último normalmente ocurre, de media, en $t=0,095 \mathrm{~s}$. Este es otro de los parámetros donde se encuentran distintos comportamientos, las máximas fuerzas en 
el ala cambian su tendencia cuando el amortiguamiento estructural cambia de los siguientes intervalos $0 \%-1,2 \%, 1,2 \%-3,6 \%$ ó $3,6 \%-5 \%$ (ver la figura 5.16 ). Dentro de cada intervalo las fuerzas decrecen cuando el amortiguamiento se incrementa. Finalmente, la variable FFE decrece cuando DAMP se incrementa.
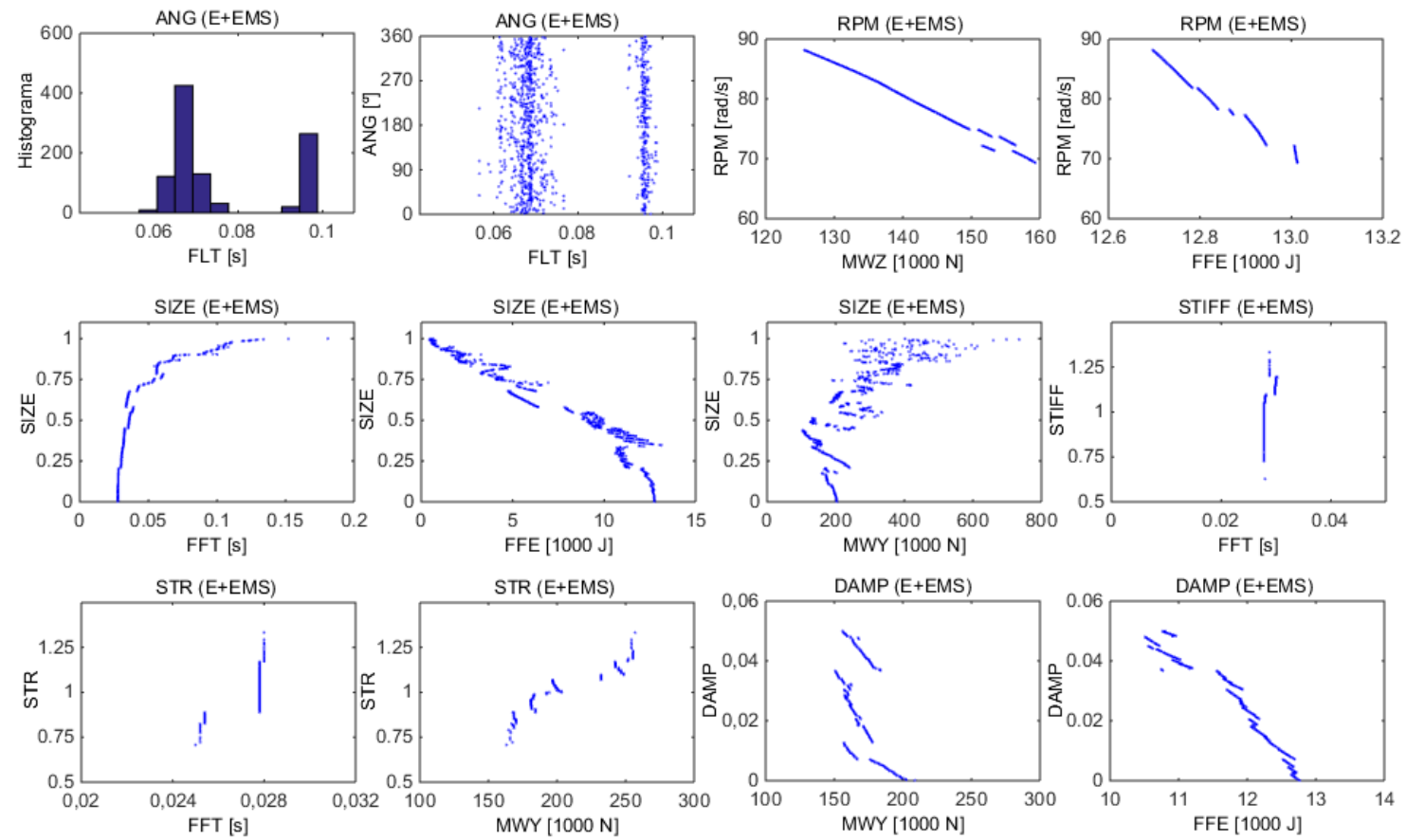

Figura 5.16: Detalle de los resultados para variaciones individuales de los parámetros
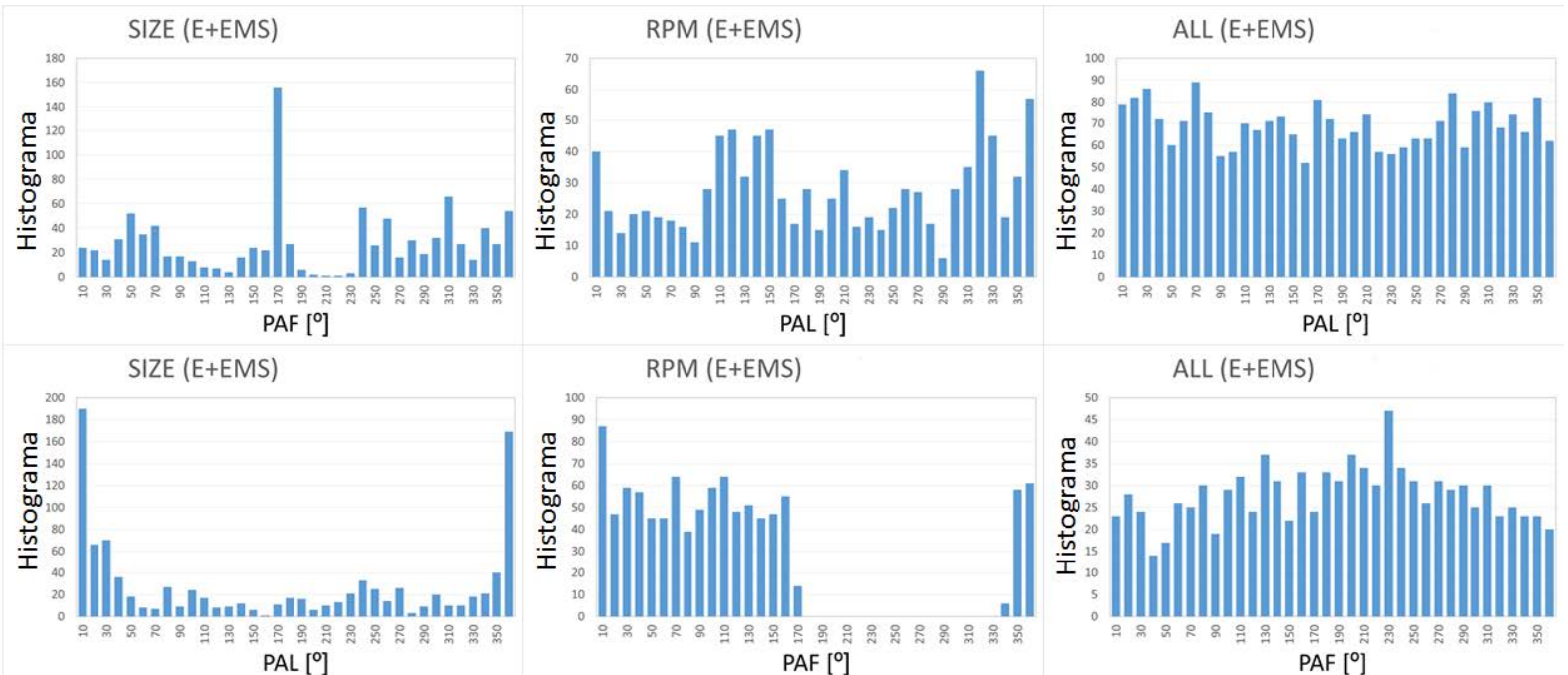

Figura 5.17: Ángulos probables de rotura para las variables SIZE, RPM y ALL en el modelo $(\mathrm{E}+\mathrm{EMS})$ 


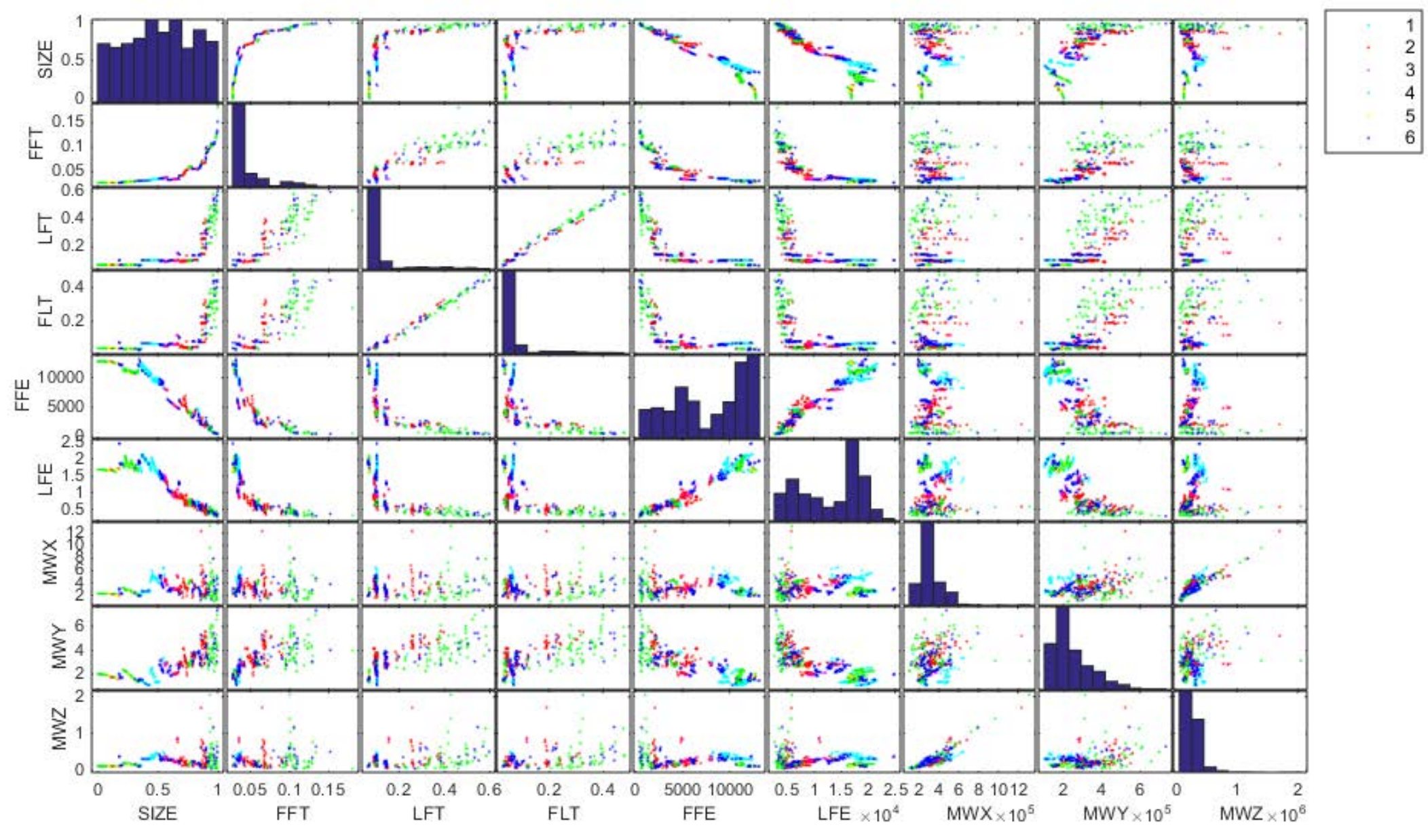

Figura 5.18: Variación de SIZE diferenciando con colores las distintas secuencias de fallo 
Figura 5.19: Variación de RPM diferenciando con colores las distintas secuencias de fallo 


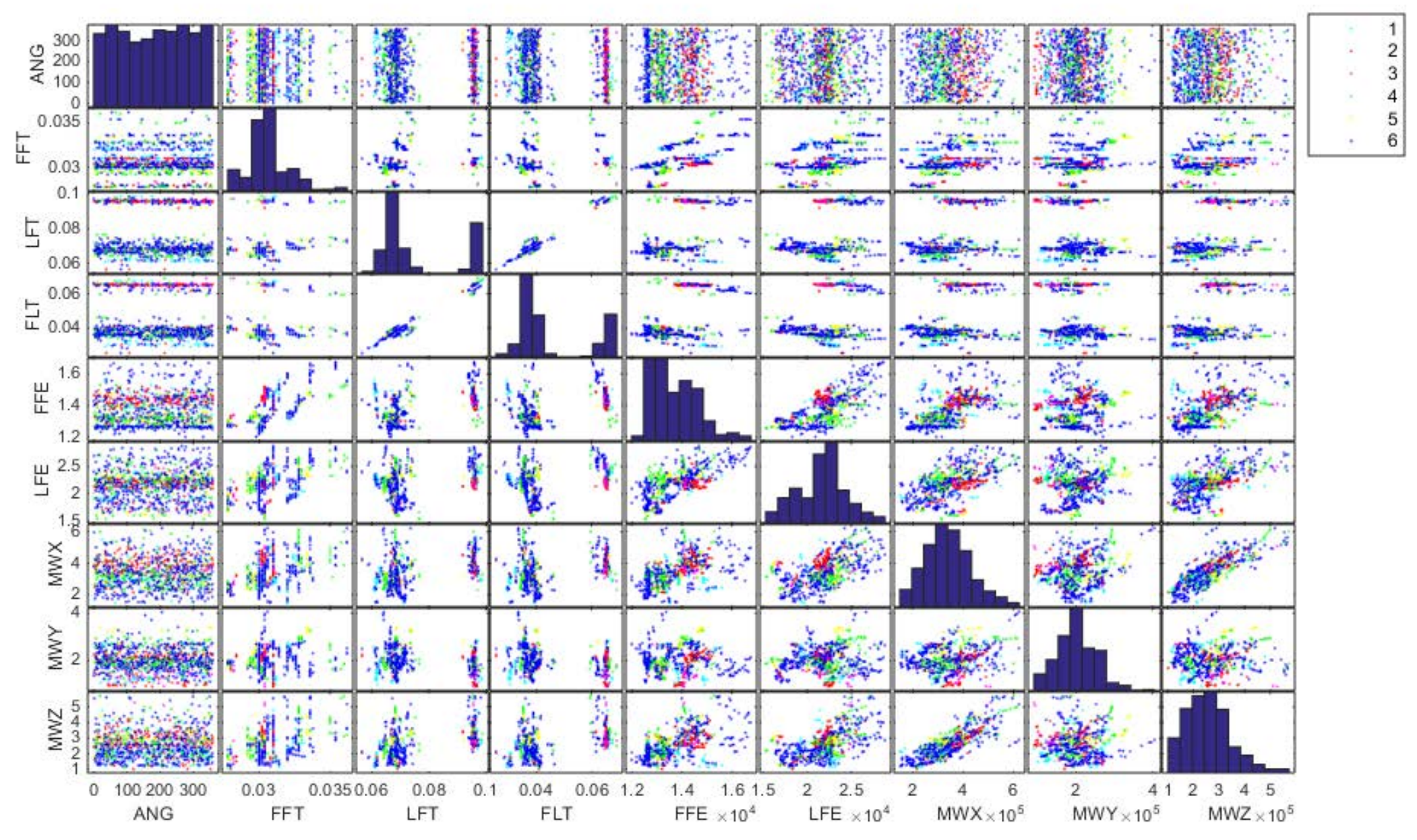

Figura 5.20: Variación de ANG diferenciando con colores las distintas secuencias de fallo 


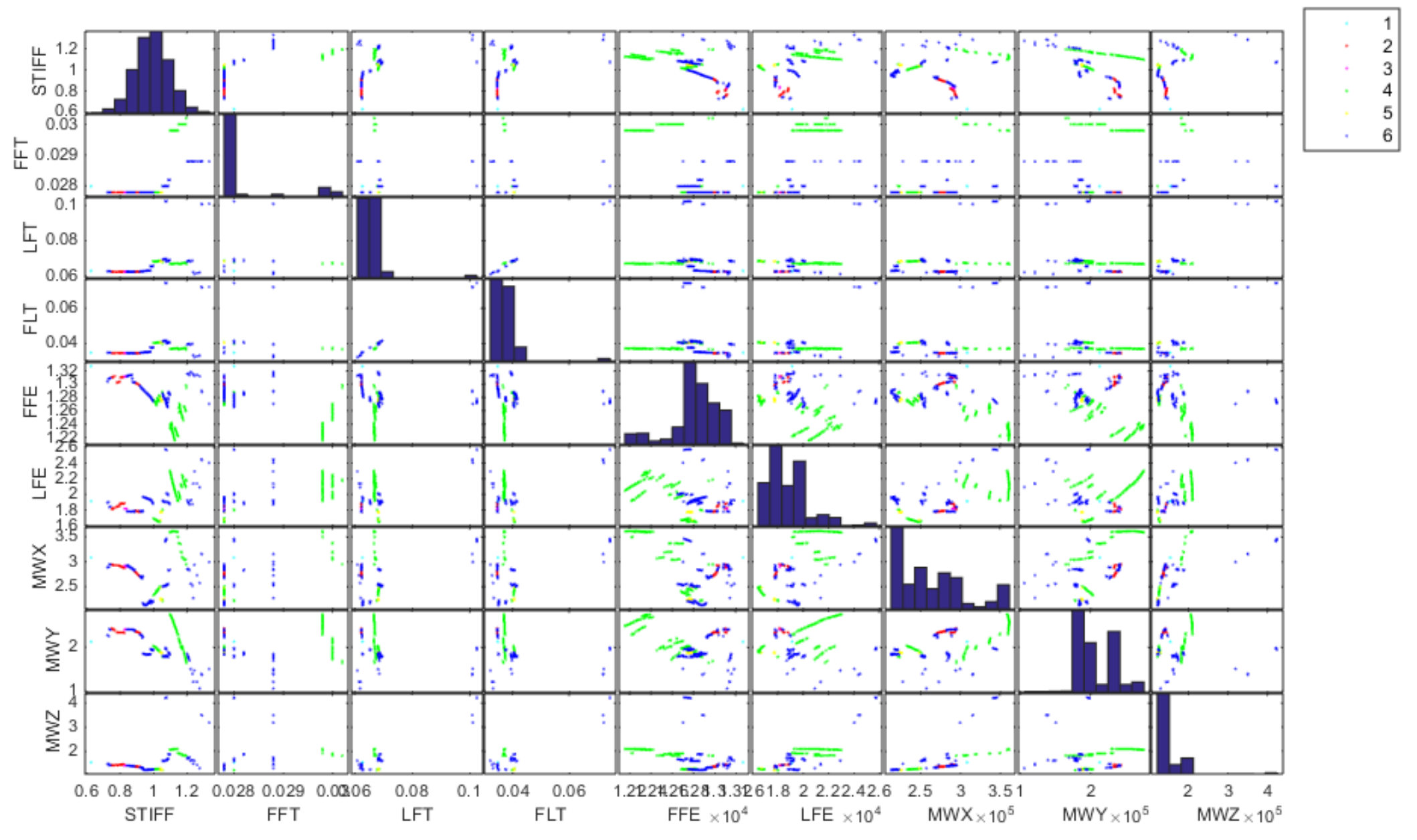

Figura 5.21: Variación de STIFF diferenciando con colores las distintas secuencias de fallo 


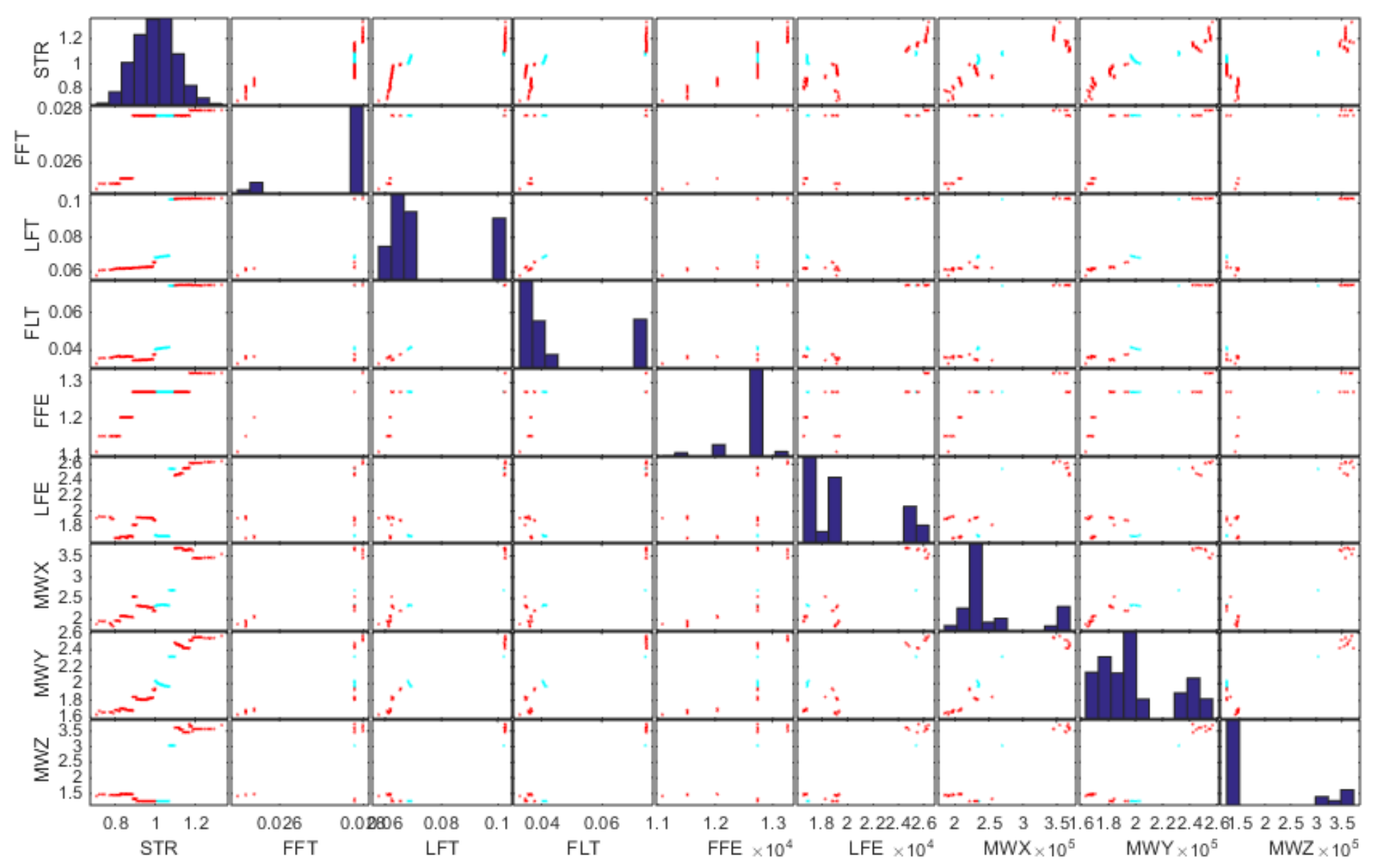

Figura 5.22: Variación de STR diferenciando con colores las distintas secuencias de fallo 


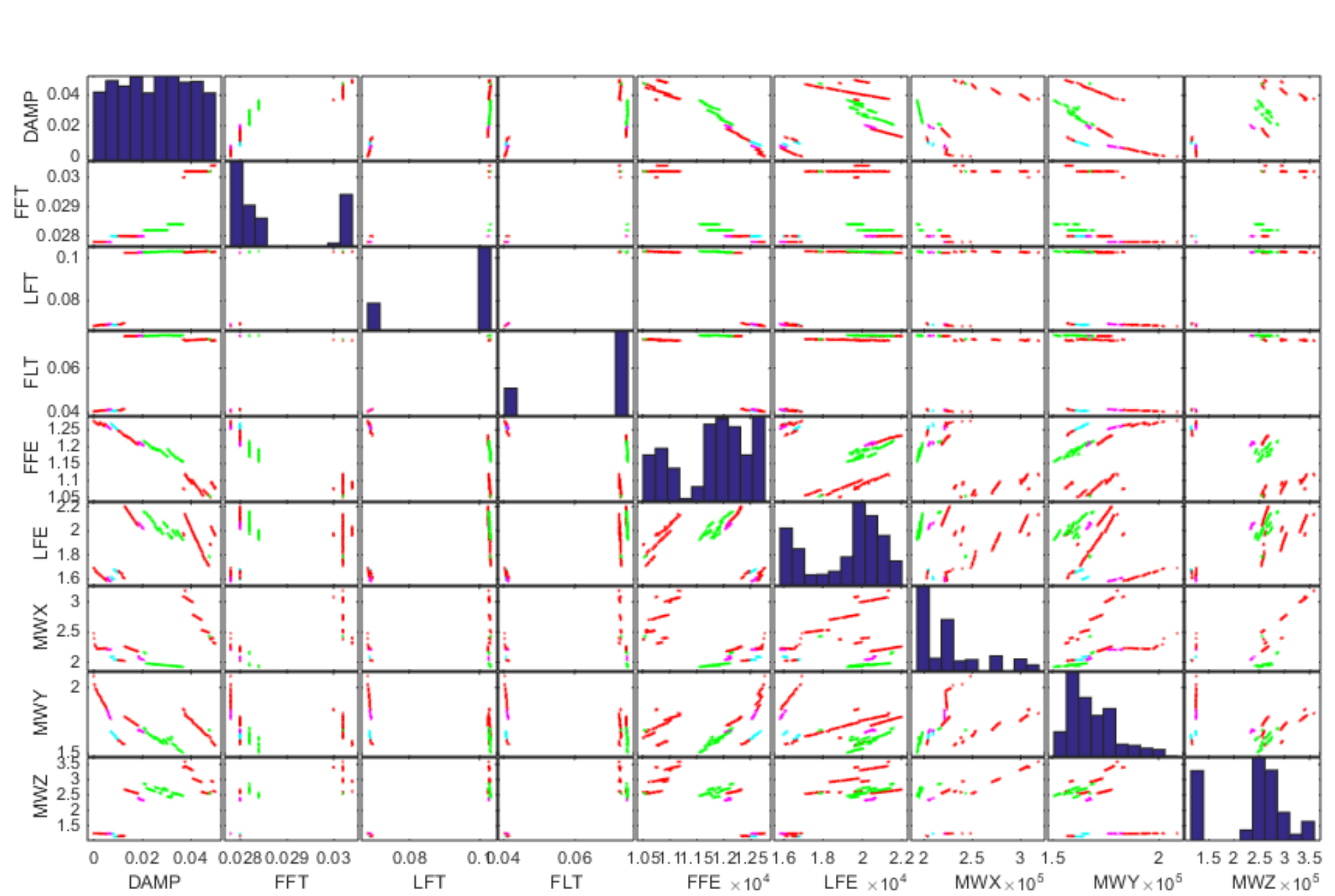

Figura 5.23: Variación de DAMP diferenciando con colores las distintas secuencias de fallo 


\subsubsection{Interacción de todos los parámetros}

Finalmente, se ha llevado a cabo un análisis entre todos los parámetros de entrada, la matriz de correlación se puede ver en la figura 5.12. En estos resultados se observan en mayor o menor medida los mostrados en los apartados anteriores y se puede discernir claramente cuáles son las variables de entrada con mayor influencia. Los resultados del análisis cuando se cambia el tamaño de la pala perdida varían mucho más a cuando se cambia la rigidez de los elastómeros o la frecuencia del motor a la que sucede la pérdida. En un rango medio de influencia se encuentra la resistencia de los elastómeros y la posición angular en que se encuentra la pala perdida cuando se separa. En la figura 5.24 se ve esta influencia para el caso de FFE vs. SIZE variando sólo SIZE, SIZE\&RPM, SIZE\&ANG, SIZE\&STIFF, SIZE\&STR y SIZE\&DAMP.
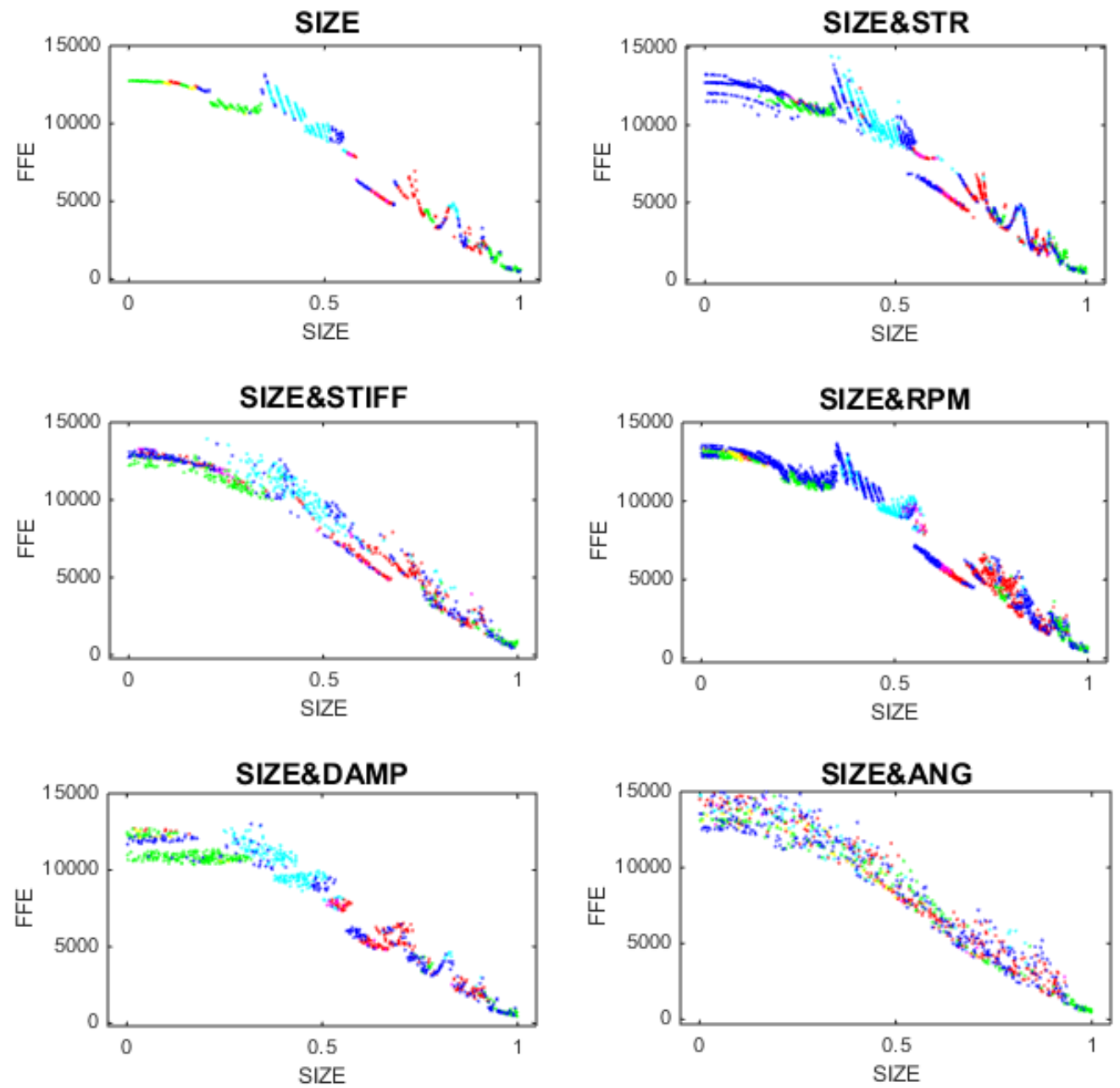

Figura 5.24: Resultados de FFE vs. SIZE variando sólo SIZE o SIZE más otro parámetro

Es importante destacar que los resultados variando una sola variable de entrada o dos variables de entrada se pueden obtener de la variación de TODO ya que si el número de simulaciones es lo suficientemente grande se pueden identificar un número suficiente de casos en 
las que las demás variables sean las nominales y sólo varíe la variable que se quiera. De hecho, como ahora mismo acabamos de decir las variables fijas de ANG, RPM, SIZE, STR, STIFF y DAMP son las nominales pero si interesan otras habría que tratar de identificar los casos que interesan en TODO y no se podría en las variaciones individuales. Por ejemplo, en la figura 5.25 se muestra el caso que se quisiera un SIZE determinado (línea roja) o un DAMP determinado (línea naranja), los puntos a los que corta la línea serían los seleccionados. Estos puntos no se están mostrando en las otras gráficas pero mediante el filtrado sería sencillo identificarlos. Por lo tanto, el grupo TODO contiene una cantidad enorme de información y con un número suficiente de casos incluye la información tanto ANG, RPM, SIZE, STR, STIFF y DAMP como de SIZE\&RPM, SIZE\&ANG, SIZE\&STIFF, SIZE\&STR y SIZE\&DAMP y de cualquier combinación.

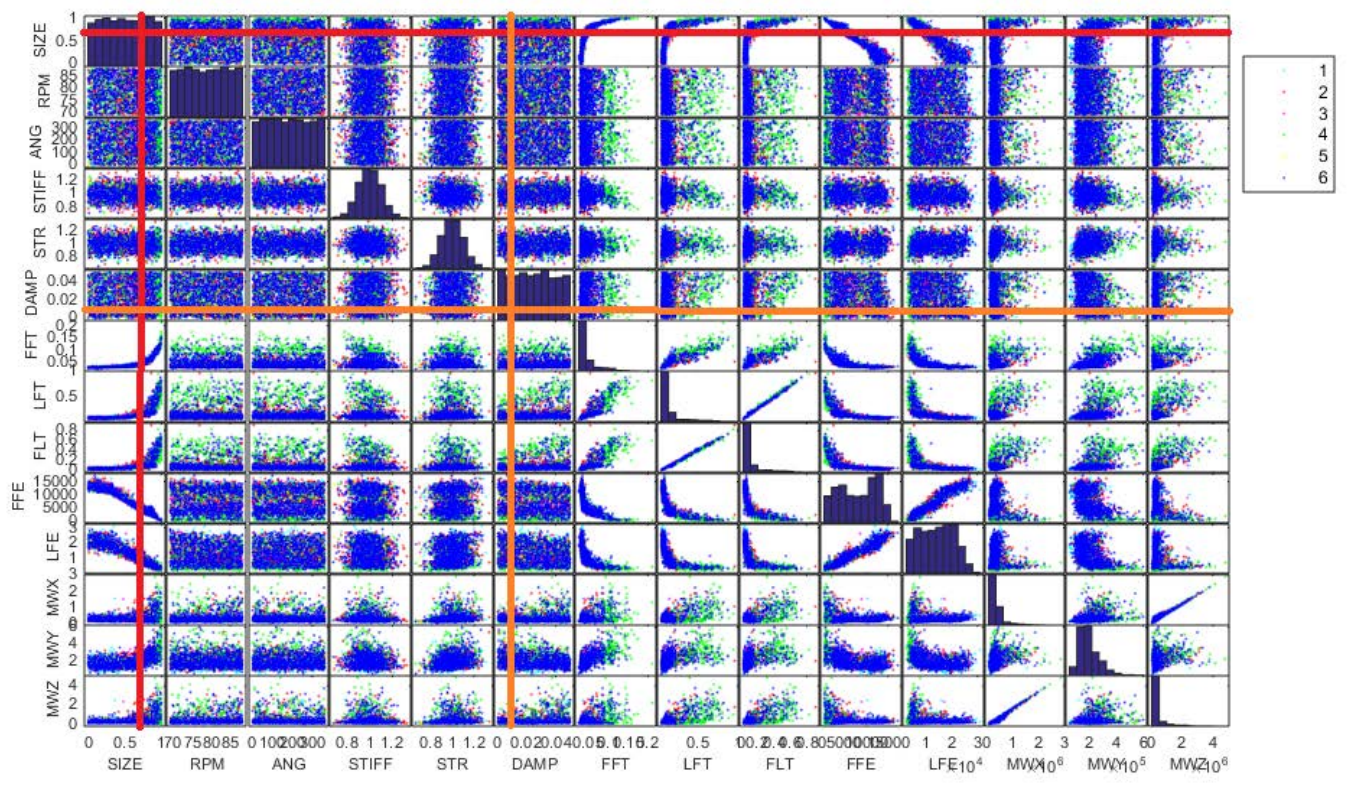

Figura 5.25: Detalle de filtrado de los resultados por SIZE (línea roja) o DAMP (línea naranja)

Aquí se pone de manifiesto la importancia crucial de la optimización que se ha llevado a cabo en el modelo para que se haya podido realizar un número hasta 10000 simulaciones que permiten identificar casi cualquier combinación de parámetros que pudiera interesar. Simplemente post-procesando los resultados de salida de TODO, filtrando los rangos de interés de la variables se obtienen la variación requerida. Esto además se puede realizar tanto en las variables de entrada como de salida y no es necesario lanzar ningún análisis adicional como sucedería en el caso del capítulo 4.

Para terminar, en las figuras 5.26, 5.27, 5.28, 5.29, 5.30 y 5.31 se muestran todos los resultados variando los parámetros SIZE\&RPM, SIZE\&ANG, SIZE\&STIFF, SIZE\&STR, SIZE\&DAMP y TODO respectivamente. 


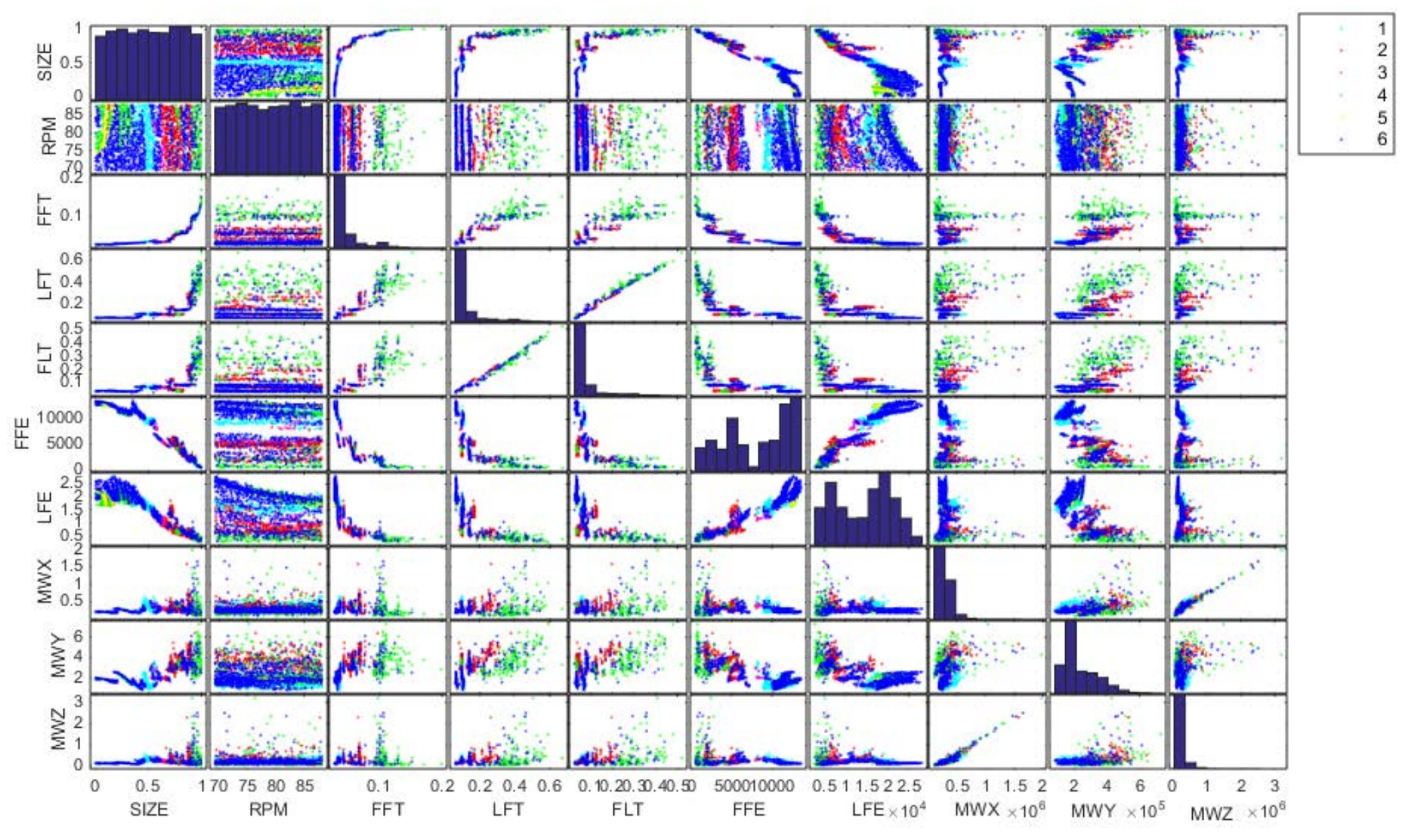

Figura 5.26: Variación de SIZE\&RPM diferenciando con colores las distintas secuencias de fallo 


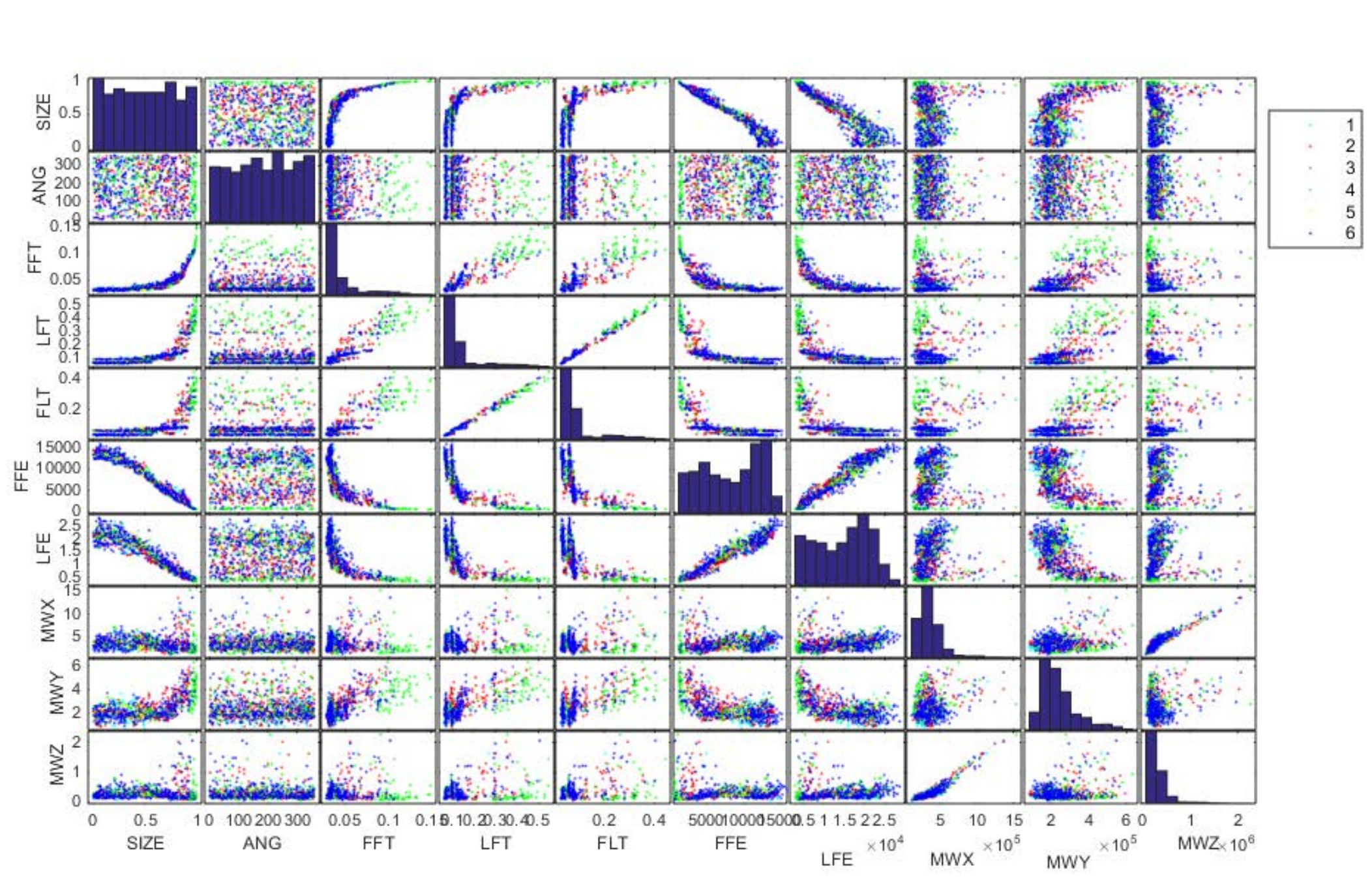

Figura 5.27: Variación de SIZE\&ANG diferenciando con colores las distintas secuencias de fallo 


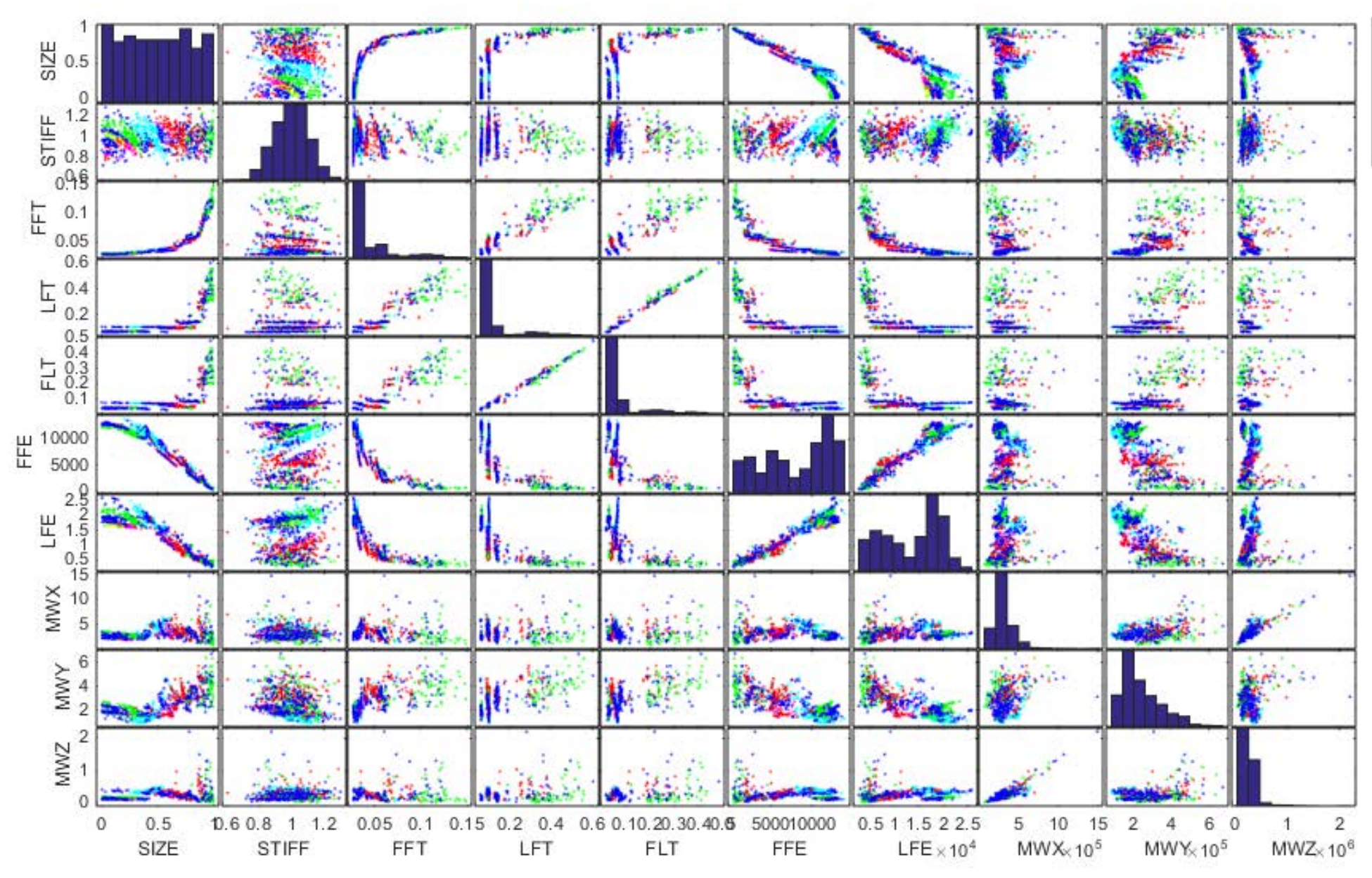

Figura 5.28: Variación de SIZE\&STIFF diferenciando con colores las distintas secuencias de fallo 


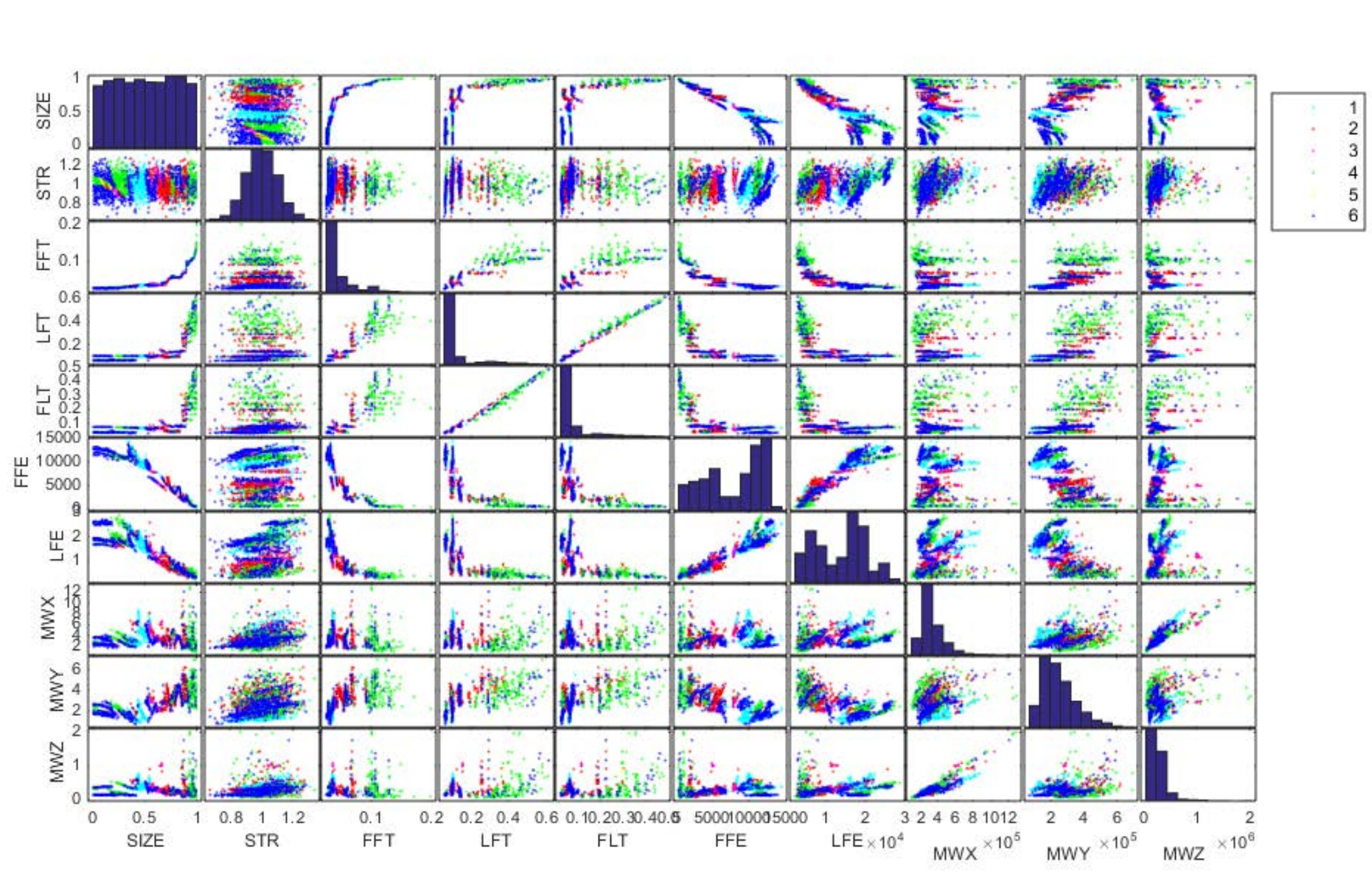

Figura 5.29: Variación de SIZE\&STR diferenciando con colores las distintas secuencias de fallo 


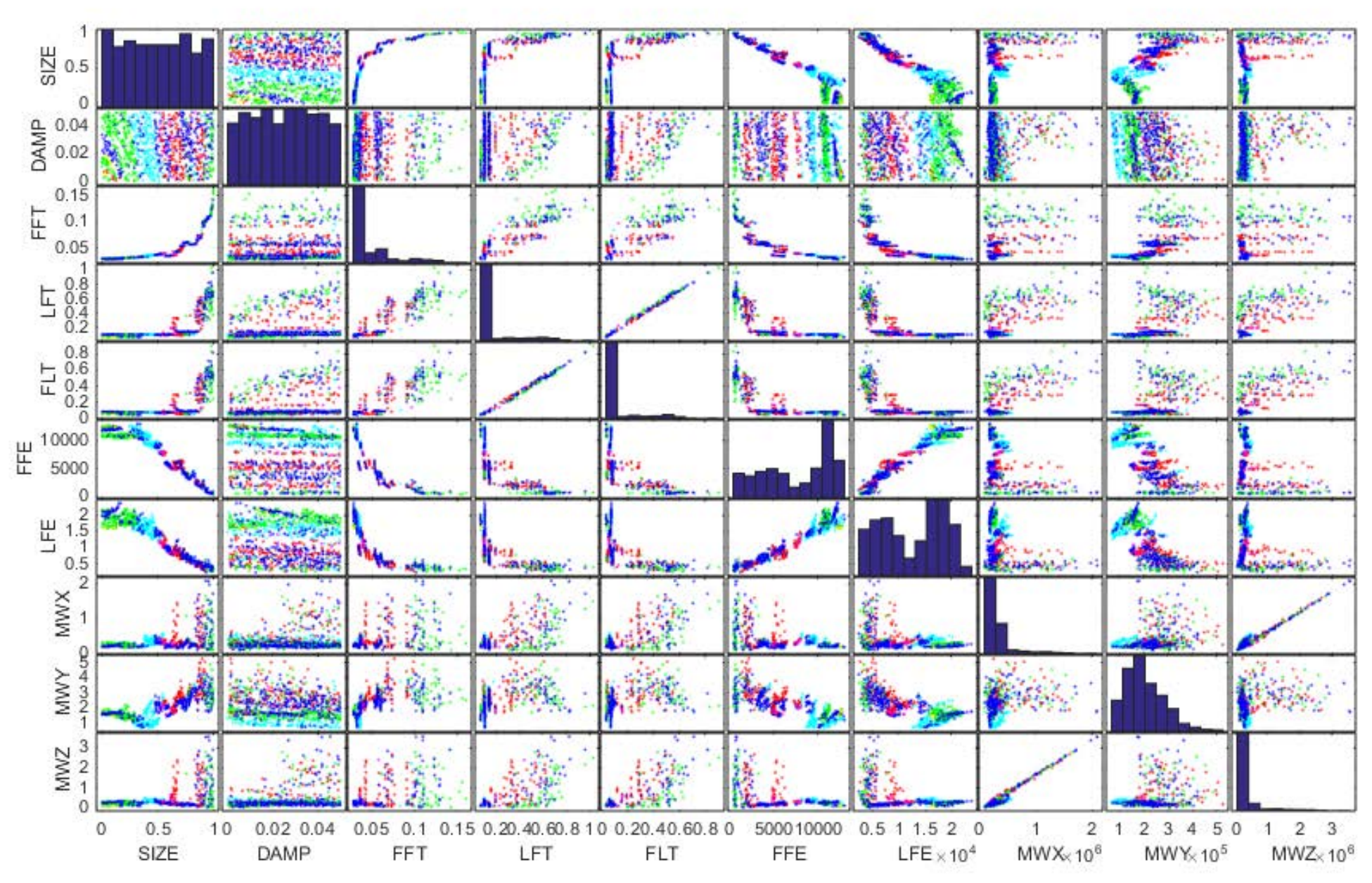

Figura 5.30: Variación de SIZE\&DAMP diferenciando con colores las distintas secuencias de fallo 


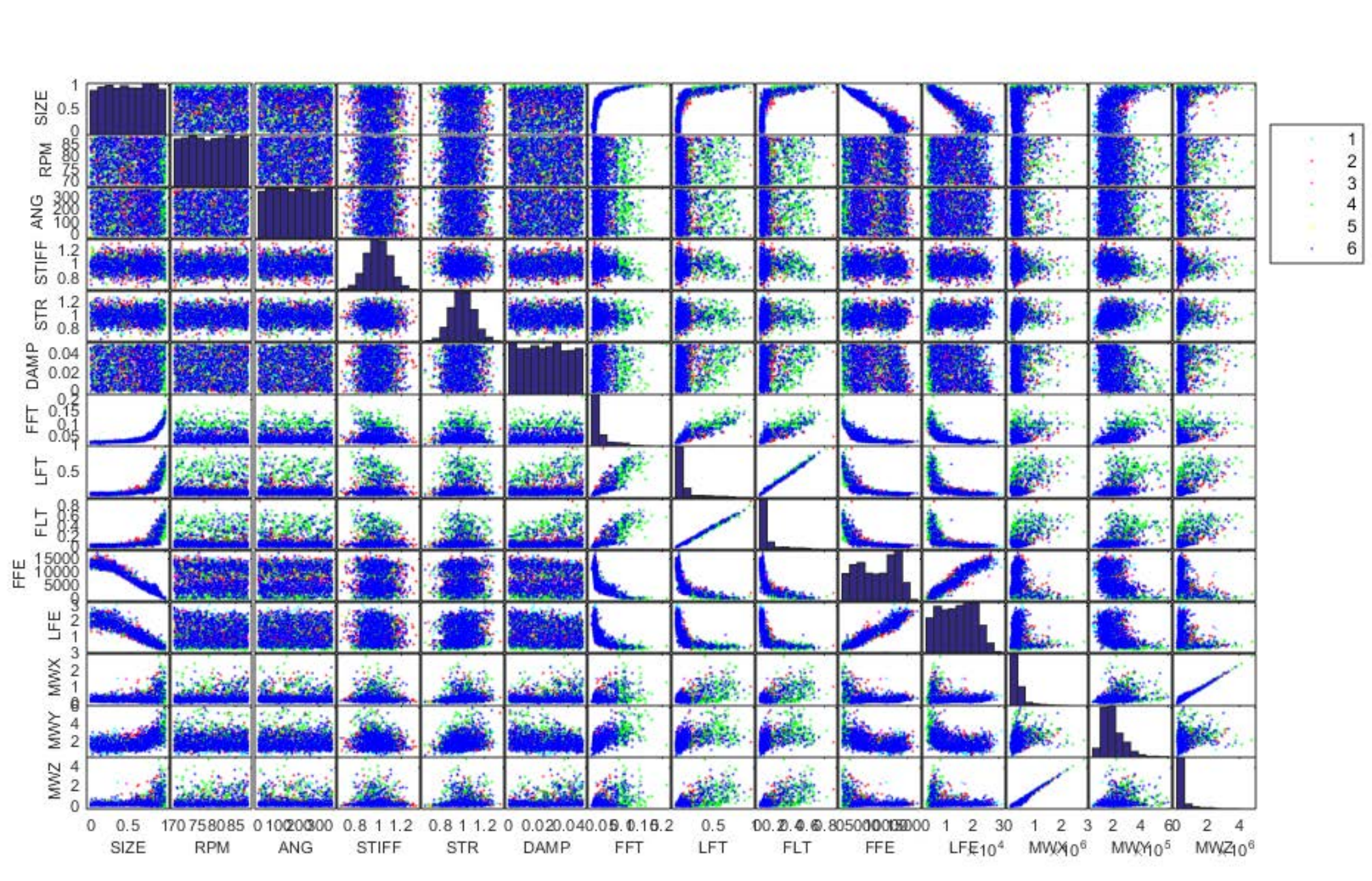

Figura 5.31: Variación de TODO diferenciando con colores las distintas secuencias de fallo 


\subsubsection{Resultados de la unión bancada-ala}

Como se ha mencionado anteriormente, el modelo es capaz de introducir la bancada (E+EMS) y, por lo tanto, calcular las fuerzas que alcanzan el ala (MWX, MWY \& MWZ). Un ejemplo se muestra en la figura 5.10, donde se ven los resultados de SIZE para E y E+EMS.

Es importante resaltar sólo se pueden obtener resultados de esta unión con el modelo (E+EMS) y no con (E). Lo que sí se puede obtener, y que también se muestran en la tabla 5.4 al comparar el mismo caso de carga con y sin bancada, es que la introducción de la bancada afecta de la siguiente manera: retrasa en tiempo la rotura de los elastómeros, acorta el tiempo entre que se produce la primera y la última rotura y los ángulos más probables en los que se producen las roturas cambian (PAF \& PAL). Además, también se advierte que la pendiente de la curva de la energía es menor.

Asimismo, un resultado que se debe destacar es que en la mayoría de las simulaciones las fuerzas máximas en el eje Y superan el valor admisible, al igual que sucedía en el capítulo 4. En la figura 5.7 se muestran los resultados de máxima fuerza por cada eje (cuadrado negro) que son a su vez uno de los miles de valores de las variables MWX, MWY \& MWZ (uno de los puntos en cada gráfica de la figura 5.32). Si los valores admisibles se ponen en el gráfico de las máximas fuerzas, se ve claramente que sólo en el caso de MWX vs. MWZ los valores se mantienen casi siempre entre los límites (ver figura 5.32). Por el contrario, MWY y, en consecuencia, la fuerza $F_{y}$, probablemente excederá de los valores límite.

De hecho, esta metodología permite el cálculo de la probabilidad de fallo para unos valores característicos de $F_{x}=600000[\mathrm{~N}], F_{y}=100000[\mathrm{~N}]$ y $F_{z}=600000$ [N]. Los valores que se obtienen con estos admisibles son del 9,84\%, $97 \%$ y 12,92\% que son las probabilidades de superar el admisible $F_{x}, F_{y}$ y $F_{z}$ respectivamente, y corresponden a las 3 primeras columnas de la tabla 5.5.

A continuación, se realiza un estudio de los admisibles necesarios para que las probabilidades de fallo sean bajas para los grupos de casos de carga (filas de la tabla). En realidad, valdría con calcularlo para el grupo ALL pero se ha calculado para todos los grupos de variaciones para ver cómo afecta cada subgrupo de variación a cada grupo de admisibles y a cada dirección. Por lo tanto se varían las fuerzas admisibles y se calculan las probabilidades de fallo. En esta tabla se han calculado las probabilidades de fallo para: $F_{x}=600000[\mathrm{~N}], F_{y}=100000[\mathrm{~N}]$ y $F_{z}=600000[\mathrm{~N}]$, para $F_{x}=100000[\mathrm{~N}], F_{y}=100000[\mathrm{~N}]$ y $F_{z}=100000[\mathrm{~N}]$, para $F_{x}=300000$ $[\mathrm{N}], F_{y}=300000[\mathrm{~N}]$ y $F_{z}=300000[\mathrm{~N}]$ y para $F_{x}=600000[\mathrm{~N}], F_{y}=400000[\mathrm{~N}]$ y $F_{z}=800000$ [N]. Finalmente, se obtiene que hay menos de un $10 \%$ de probabilidad de fallo en cada dirección si sus admisibles son $F_{x}=600000[\mathrm{~N}], F_{y}=400000[\mathrm{~N}]$ y $F_{z}=800000[\mathrm{~N}]$, que corresponden a las 3 últimas columnas de la tabla 5.5. 


\begin{tabular}{|c|c|c|c|c|c|c|c|c|c|c|c|c|}
\hline & $F_{x}[N]$ & $F_{y}[N]$ & $F_{z}[N]$ & $F_{x}[N]$ & $F_{y}[N]$ & $F_{z}[N]$ & $F_{x}[N]$ & $F_{y}[N]$ & $F_{z}[N]$ & $F_{x}[N]$ & $F_{y}[N]$ & $F_{z}[N]$ \\
& 600000 & 100000 & 600000 & 100000 & 100000 & 100000 & 300000 & 300000 & 300000 & 600000 & 400000 & 800000 \\
\hline ANG & $0,7 \%$ & $98,2 \%$ & $0,0 \%$ & $100,0 \%$ & $98,2 \%$ & $100,0 \%$ & $65,1 \%$ & $2,5 \%$ & $27,3 \%$ & $0,7 \%$ & $0,1 \%$ & $0,0 \%$ \\
\hline RPM & $0,0 \%$ & $100,0 \%$ & $0,0 \%$ & $100,0 \%$ & $100,0 \%$ & $100,0 \%$ & $0,0 \%$ & $0,0 \%$ & $0,0 \%$ & $0,0 \%$ & $0,0 \%$ & $0,0 \%$ \\
\hline SIZE & $1,3 \%$ & $100,0 \%$ & $2,5 \%$ & $100,0 \%$ & $100,0 \%$ & $98,0 \%$ & $41,5 \%$ & $23,1 \%$ & $30,7 \%$ & $1,3 \%$ & $8,3 \%$ & $1,0 \%$ \\
\hline STIFF & $0,0 \%$ & $100,0 \%$ & $0,0 \%$ & $100,0 \%$ & $100,0 \%$ & $100,0 \%$ & $14,3 \%$ & $0,0 \%$ & $1,4 \%$ & $0,0 \%$ & $0,0 \%$ & $0,0 \%$ \\
\hline STR & $0,0 \%$ & $100,0 \%$ & $0,0 \%$ & $100,0 \%$ & $100,0 \%$ & $100,0 \%$ & $17,4 \%$ & $0,0 \%$ & $24,8 \%$ & $0,0 \%$ & $0,0 \%$ & $0,0 \%$ \\
\hline DAMP & $0,0 \%$ & $100,0 \%$ & $0,0 \%$ & $100,0 \%$ & $100,0 \%$ & $100,0 \%$ & $8,2 \%$ & $0,0 \%$ & $11,6 \%$ & $0,0 \%$ & $0,0 \%$ & $0,0 \%$ \\
\hline SIZE\&ANG & $5,5 \%$ & $99,0 \%$ & $6,5 \%$ & $100,0 \%$ & $99,0 \%$ & $98,0 \%$ & $59,6 \%$ & $20,0 \%$ & $34,7 \%$ & $5,5 \%$ & $7,5 \%$ & $3,7 \%$ \\
\hline SIZE\&RPM & $2,1 \%$ & $98,4 \%$ & $2,7 \%$ & $100,0 \%$ & $98,4 \%$ & $97,2 \%$ & $32,9 \%$ & $24,1 \%$ & $18,6 \%$ & $2,1 \%$ & $7,5 \%$ & $1,7 \%$ \\
\hline SIZE\&STIFF & $2,4 \%$ & $98,9 \%$ & $2,4 \%$ & $99,9 \%$ & $98,9 \%$ & $96,7 \%$ & $43,2 \%$ & $25,8 \%$ & $30,6 \%$ & $2,4 \%$ & $8,9 \%$ & $1,4 \%$ \\
\hline SIZE\&DAMP & $8,4 \%$ & $92,2 \%$ & $11,1 \%$ & $99,8 \%$ & $92,2 \%$ & $98,9 \%$ & $34,5 \%$ & $12,6 \%$ & $37,8 \%$ & $8,4 \%$ & $2,1 \%$ & $9,4 \%$ \\
\hline ALL & $9,8 \%$ & $97,0 \%$ & $12,9 \%$ & $99,5 \%$ & $97,0 \%$ & $98,4 \%$ & $47,1 \%$ & $9,7 \%$ & $41,2 \%$ & $9,8 \%$ & $2,2 \%$ & $9,7 \%$ \\
\hline
\end{tabular}

Tabla 5.5: Probabilidad de fallo en los apoyos para cada grupo de casos de carga

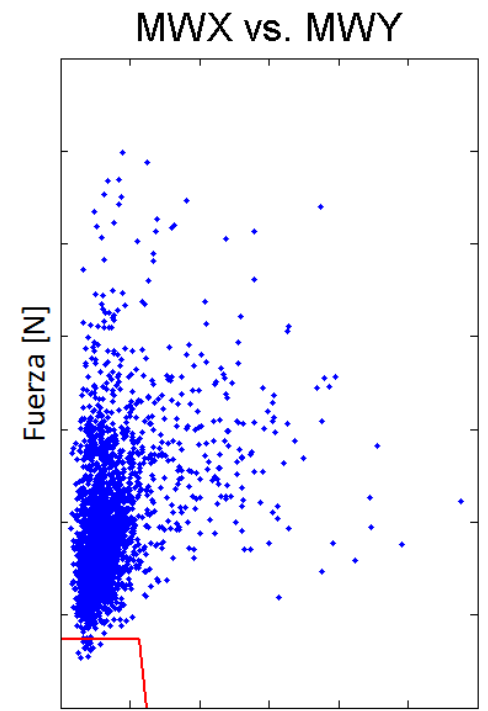

Fuerza $[\mathrm{N}]$

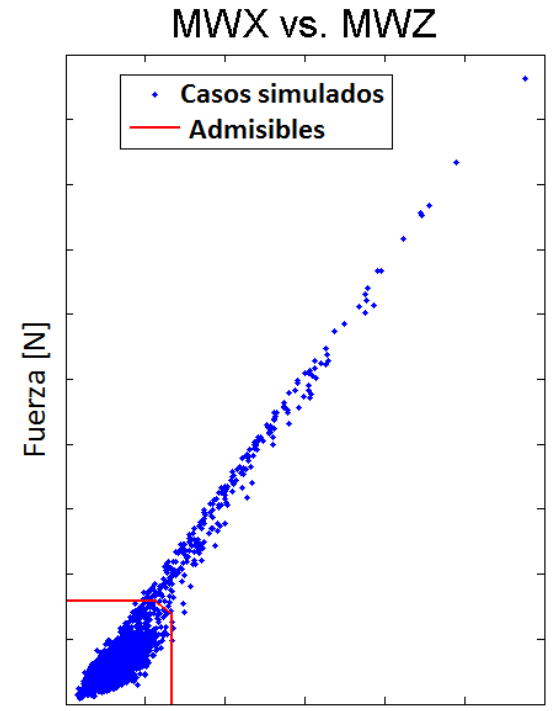

Fuerza [N]
MWY vs. MWZ

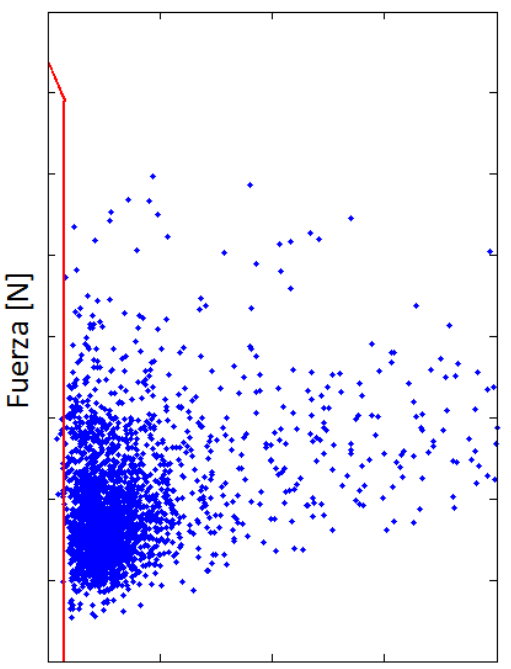

Fuerza $[\mathrm{N}]$

Figura 5.32: Fuerzas máximas en el ala y los límites admisibles

\subsubsection{Resultados de la secuencia de fallo}

En este caso se ha realizado un estudio pormenorizado de las posibles secuencias de fallo de los distintos apoyos entre la bancada y el motor. La secuencia de fallo se ha denominado mediante un número de seis cifras en donde el primer número, que puede ser del 1 al 6 , indica el momento en la secuencia que ha fallado el apoyo $\mathrm{TOP}+$, el segundo número, que puede ser del 1 al 6, indica el momento en la secuencia que ha fallado el apoyo TOP-, el tercer número, que puede ser del 1 al 6, indica el momento en la secuencia que ha fallado el apoyo SIDE+, el cuarto número, que puede ser del 1 al 6 , indica el momento en la secuencia que ha fallado el apoyo SIDE-, el quinto número, que puede ser del 1 al 6, indica el momento en la secuencia que ha fallado el apoyo REAR+ y el sexto número, que puede ser del 1 al 6 , indica el momento en 
la secuencia que ha fallado el apoyo REAR-. Por lo tanto si la secuencia es 123456 indica que el orden de fallo ha sido TOP+, TOP-, SIDE+, SIDE-, REAR+ y REAR-. En la tabla 5.6 se indica que porcentaje de simulaciones se ha producido con una determinada secuencia de fallo. Hay que tener en cuenta que como se ha permitido que los apoyos fallen de forma simultánea sucede que hay casos que se cuentan dos o más veces ya que cuando los apoyos fallan a la vez (en el mismo paso de tiempo) se cuentan indistintamente en uno u otro orden.

\begin{tabular}{|c|c|c|c|c|c|c|c|c|c|c|c|c|}
\hline ORDEN & TODO & $D A M P$ & $S T R$ & STIFF & $R P M$ & $S I Z E$ & $A N G$ & SIZE\&STR & SIZE\&DAMP & $S I Z E \& R P M$ & $S I Z E \& S T I F F$ & $S I Z E \& A N G$ \\
\hline 123456 & $2,00 \%$ & $0,00 \%$ & $0,00 \%$ & $0,30 \%$ & $0,00 \%$ & $1,48 \%$ & $1,30 \%$ & $1,76 \%$ & $3,80 \%$ & $5,12 \%$ & $1,68 \%$ & $2,52 \%$ \\
\hline 123465 & $0,92 \%$ & $0,00 \%$ & $6,10 \%$ & $0,30 \%$ & $0,00 \%$ & $1,68 \%$ & $1,20 \%$ & $2,12 \%$ & $0,50 \%$ & $2,24 \%$ & $0,98 \%$ & $0,92 \%$ \\
\hline 124356 & $2,72 \%$ & $0,00 \%$ & $0,10 \%$ & $0,30 \%$ & $0,00 \%$ & $7,60 \%$ & $2,00 \%$ & $7,20 \%$ & $10,70 \%$ & $11,24 \%$ & $8,54 \%$ & $2,96 \%$ \\
\hline 124365 & $0,80 \%$ & $0,00 \%$ & $13,40 \%$ & $0,30 \%$ & $0,00 \%$ & $1,68 \%$ & $1,10 \%$ & $3,68 \%$ & $0,50 \%$ & $2,28 \%$ & $1,02 \%$ & $0,84 \%$ \\
\hline 132456 & $0,44 \%$ & $0,00 \%$ & $0,00 \%$ & $0,00 \%$ & $0,00 \%$ & $0,00 \%$ & $0,20 \%$ & $0,00 \%$ & $0,00 \%$ & $0,00 \%$ & $0,00 \%$ & $1,08 \%$ \\
\hline 132465 & $0,28 \%$ & $0,00 \%$ & $0,00 \%$ & $0,10 \%$ & $0,00 \%$ & $0,00 \%$ & $0,00 \%$ & $0,00 \%$ & $0,00 \%$ & $0,00 \%$ & $0,00 \%$ & $0,16 \%$ \\
\hline 134256 & $0,60 \%$ & $0,00 \%$ & $0,00 \%$ & $1,20 \%$ & $0,00 \%$ & $0,00 \%$ & $1,20 \%$ & $0,00 \%$ & $0,00 \%$ & $0,00 \%$ & $0,20 \%$ & $1,32 \%$ \\
\hline 134265 & $0,32 \%$ & $0,00 \%$ & $0,00 \%$ & $0,00 \%$ & $0,00 \%$ & $0,00 \%$ & $0,00 \%$ & $0,00 \%$ & $0,00 \%$ & $0,00 \%$ & $0,00 \%$ & $0,08 \%$ \\
\hline 142356 & $0,16 \%$ & $0,00 \%$ & $0,00 \%$ & $0,00 \%$ & $0,00 \%$ & $0,00 \%$ & $0,00 \%$ & $0,00 \%$ & $0,00 \%$ & $0,00 \%$ & $0,00 \%$ & $0,40 \%$ \\
\hline 142365 & $0,04 \%$ & $0,00 \%$ & $0,00 \%$ & $0,00 \%$ & $0,00 \%$ & $0,00 \%$ & $0,00 \%$ & $0,00 \%$ & $0,00 \%$ & $0,00 \%$ & $0,00 \%$ & $0,08 \%$ \\
\hline 143256 & $0,76 \%$ & $0,00 \%$ & $0,00 \%$ & $1,10 \%$ & $0,00 \%$ & $0,00 \%$ & $1,30 \%$ & $0,00 \%$ & $0,00 \%$ & $0,00 \%$ & $0,14 \%$ & $1,32 \%$ \\
\hline 143265 & $0,28 \%$ & $0,00 \%$ & $0,00 \%$ & $0,00 \%$ & $0,00 \%$ & $0,00 \%$ & $0,00 \%$ & $0,00 \%$ & $0,00 \%$ & $0,00 \%$ & $0,00 \%$ & $0,08 \%$ \\
\hline 213456 & $6,72 \%$ & $0,00 \%$ & $0,00 \%$ & $0,10 \%$ & $0,00 \%$ & $5,32 \%$ & $4,70 \%$ & $6,96 \%$ & $5,50 \%$ & $2,80 \%$ & $5,32 \%$ & $7,44 \%$ \\
\hline 213465 & $3,24 \%$ & $0,00 \%$ & $0,00 \%$ & $0,30 \%$ & $0,00 \%$ & $1,60 \%$ & $3,10 \%$ & $2,04 \%$ & $1,10 \%$ & $1,64 \%$ & $1,36 \%$ & $3,76 \%$ \\
\hline 214356 & $11,12 \%$ & $0,00 \%$ & $0,00 \%$ & $0,10 \%$ & $0,00 \%$ & $18,32 \%$ & $9,10 \%$ & $19,12 \%$ & $20,20 \%$ & $12,72 \%$ & $19,40 \%$ & $11,48 \%$ \\
\hline 214365 & $3,88 \%$ & $0,00 \%$ & $0,00 \%$ & $0,30 \%$ & $0,00 \%$ & $1,60 \%$ & $6,40 \%$ & $2,68 \%$ & $1,20 \%$ & $1,60 \%$ & $1,58 \%$ & $4,40 \%$ \\
\hline 231456 & $3,56 \%$ & $0,00 \%$ & $0,00 \%$ & $0,00 \%$ & $0,00 \%$ & $1,52 \%$ & $6,40 \%$ & $2,68 \%$ & $0,90 \%$ & $1,08 \%$ & $2,26 \%$ & $4,32 \%$ \\
\hline 231465 & $1,48 \%$ & $0,00 \%$ & $0,00 \%$ & $0,20 \%$ & $0,00 \%$ & $0,48 \%$ & $0,00 \%$ & $1,08 \%$ & $0,70 \%$ & $0,44 \%$ & $0,42 \%$ & $1,36 \%$ \\
\hline 234156 & $27,32 \%$ & $5,60 \%$ & $0,00 \%$ & $12,10 \%$ & $0,00 \%$ & $27,00 \%$ & $19,50 \%$ & $26,12 \%$ & $20,90 \%$ & $21,32 \%$ & $28,52 \%$ & $29,36 \%$ \\
\hline 234165 & $10,92 \%$ & $0,00 \%$ & $31,10 \%$ & $48,10 \%$ & $0,00 \%$ & $6,60 \%$ & $1,80 \%$ & $11,96 \%$ & $9,30 \%$ & $8,48 \%$ & $13,58 \%$ & $8,76 \%$ \\
\hline 241356 & $6,80 \%$ & $0,00 \%$ & $0,00 \%$ & $0,00 \%$ & $0,00 \%$ & $7,88 \%$ & $9,40 \%$ & $8,80 \%$ & $6,80 \%$ & $7,76 \%$ & $6,18 \%$ & $7,96 \%$ \\
\hline 241365 & $2,36 \%$ & $0,00 \%$ & $0,00 \%$ & $0,60 \%$ & $0,00 \%$ & $0,48 \%$ & $3,70 \%$ & $1,48 \%$ & $0,60 \%$ & $0,36 \%$ & $0,44 \%$ & $2,40 \%$ \\
\hline 243156 & $29,20 \%$ & $5,60 \%$ & $0,00 \%$ & $14,10 \%$ & $0,00 \%$ & $28,00 \%$ & $23,30 \%$ & $28,28 \%$ & $22,20 \%$ & $23,12 \%$ & $30,96 \%$ & $29,92 \%$ \\
\hline 243165 & $10,96 \%$ & $0,00 \%$ & $31,10 \%$ & $50,50 \%$ & $0,00 \%$ & $6,64 \%$ & $2,50 \%$ & $12,56 \%$ & $8,90 \%$ & $8,08 \%$ & $14,08 \%$ & $8,72 \%$ \\
\hline 312456 & $0,76 \%$ & $0,00 \%$ & $0,00 \%$ & $0,00 \%$ & $0,00 \%$ & $0,00 \%$ & $1,20 \%$ & $0,00 \%$ & $0,00 \%$ & $0,00 \%$ & $0,00 \%$ & $0,48 \%$ \\
\hline 312465 & $0,20 \%$ & $0,00 \%$ & $0,00 \%$ & $0,00 \%$ & $0,00 \%$ & $0,00 \%$ & $0,20 \%$ & $0,00 \%$ & $0,00 \%$ & $0,04 \%$ & $0,00 \%$ & $0,12 \%$ \\
\hline 314256 & $0,12 \%$ & $0,00 \%$ & $0,00 \%$ & $0,00 \%$ & $0,00 \%$ & $0,00 \%$ & $0,40 \%$ & $0,00 \%$ & $0,00 \%$ & $0,00 \%$ & $0,02 \%$ & $0,28 \%$ \\
\hline 321456 & $0,84 \%$ & $0,00 \%$ & $0,00 \%$ & $0,00 \%$ & $0,00 \%$ & $0,00 \%$ & $3,00 \%$ & $0,00 \%$ & $0,00 \%$ & $0,00 \%$ & $0,04 \%$ & $1,76 \%$ \\
\hline 321465 & $0,48 \%$ & $0,00 \%$ & $0,00 \%$ & $0,00 \%$ & $0,00 \%$ & $0,00 \%$ & $0,40 \%$ & $0,00 \%$ & $0,00 \%$ & $0,16 \%$ & $0,02 \%$ & $0,12 \%$ \\
\hline 324156 & $3,04 \%$ & $5,60 \%$ & $0,00 \%$ & $8,40 \%$ & $0,00 \%$ & $4,16 \%$ & $3,40 \%$ & $1,68 \%$ & $1,30 \%$ & $2,00 \%$ & $3,62 \%$ & $4,08 \%$ \\
\hline 324165 & $1,72 \%$ & $0,00 \%$ & $31,10 \%$ & $44,20 \%$ & $0,00 \%$ & $0,16 \%$ & $0,30 \%$ & $4,32 \%$ & $0,20 \%$ & $0,52 \%$ & $4,68 \%$ & $1,64 \%$ \\
\hline 341256 & $4,92 \%$ & $38,60 \%$ & $10,00 \%$ & $17,80 \%$ & $17,10 \%$ & $14,16 \%$ & $5,60 \%$ & $14,76 \%$ & $21,40 \%$ & $15,08 \%$ & $14,76 \%$ & $5,44 \%$ \\
\hline 341265 & $1,80 \%$ & $0,00 \%$ & $0,00 \%$ & $0,00 \%$ & $0,00 \%$ & $0,36 \%$ & $0,90 \%$ & $0,44 \%$ & $0,10 \%$ & $5,80 \%$ & $0,86 \%$ & $1,68 \%$ \\
\hline 342156 & $21,56 \%$ & \begin{tabular}{|l|}
$58,00 \%$ \\
\end{tabular} & $34,80 \%$ & $39,80 \%$ & $26,20 \%$ & $37,40 \%$ & $22,20 \%$ & $24,68 \%$ & $29,80 \%$ & $20,24 \%$ & $27,10 \%$ & $23,88 \%$ \\
\hline 342165 & $9,44 \%$ & $0,00 \%$ & $34,40 \%$ & $46,10 \%$ & $47,00 \%$ & $3,72 \%$ & $2,30 \%$ & $8,64 \%$ & $6,80 \%$ & $7,00 \%$ & $10,80 \%$ & $9,68 \%$ \\
\hline 412356 & $0,12 \%$ & $0,00 \%$ & $0,00 \%$ & $0,00 \%$ & $0,00 \%$ & $0,00 \%$ & $0,00 \%$ & $0,00 \%$ & $0,10 \%$ & $0,00 \%$ & $0,00 \%$ & $0,24 \%$ \\
\hline 412365 & $0,00 \%$ & $0,00 \%$ & $0,00 \%$ & $0,00 \%$ & $0,00 \%$ & $0,04 \%$ & $0,10 \%$ & $0,00 \%$ & $0,00 \%$ & $0,04 \%$ & $0,00 \%$ & $0,04 \%$ \\
\hline 413256 & $0,28 \%$ & $0,00 \%$ & $0,00 \%$ & $0,00 \%$ & $0,00 \%$ & $0,00 \%$ & $0,20 \%$ & $0,00 \%$ & $0,00 \%$ & $0,00 \%$ & $0,04 \%$ & $0,48 \%$ \\
\hline 421356 & $0,20 \%$ & $0,00 \%$ & $0,00 \%$ & $0,00 \%$ & $0,00 \%$ & $0,00 \%$ & $0,00 \%$ & $0,00 \%$ & $0,00 \%$ & $0,00 \%$ & $0,00 \%$ & $0,44 \%$ \\
\hline 421365 & $0,12 \%$ & $0,00 \%$ & $0,00 \%$ & $0,00 \%$ & $0,00 \%$ & $0,04 \%$ & $0,20 \%$ & $0,04 \%$ & $0,00 \%$ & $0,00 \%$ & $0,00 \%$ & $0,32 \%$ \\
\hline 423156 & $3,76 \%$ & $10,20 \%$ & $0,00 \%$ & $10,50 \%$ & $6,60 \%$ & $7,24 \%$ & $5,90 \%$ & $3,28 \%$ & $2,30 \%$ & $4,40 \%$ & $5,90 \%$ & $4,32 \%$ \\
\hline 423165 & $1,56 \%$ & $0,00 \%$ & $31,10 \%$ & $46,20 \%$ & $6,60 \%$ & $0,16 \%$ & $1,30 \%$ & $5,04 \%$ & $0,20 \%$ & $0,84 \%$ & $5,12 \%$ & $1,72 \%$ \\
\hline 431256 & $5,40 \%$ & \begin{tabular}{|l|}
$59,30 \%$ \\
\end{tabular} & $17,40 \%$ & $22,40 \%$ & $17,10 \%$ & $16,64 \%$ & $6,90 \%$ & $17,60 \%$ & $24,20 \%$ & $19,60 \%$ & $16,36 \%$ & $6,00 \%$ \\
\hline 431265 & $1,92 \%$ & $0,00 \%$ & $0,00 \%$ & $0,00 \%$ & $0,00 \%$ & $0,36 \%$ & $0,90 \%$ & $0,44 \%$ & $0,10 \%$ & $6,68 \%$ & $0,84 \%$ & $1,52 \%$ \\
\hline 432156 & $24,24 \%$ & $66,20 \%$ & $34,80 \%$ & $44,00 \%$ & $32,80 \%$ & $40,44 \%$ & $28,50 \%$ & $25,80 \%$ & $31,20 \%$ & $22,64 \%$ & $28,32 \%$ & $27,16 \%$ \\
\hline 432165 & $9,24 \%$ & $0,00 \%$ & $34,40 \%$ & $46,10 \%$ & $61,20 \%$ & $3,68 \%$ & $2,80 \%$ & $8,80 \%$ & $6,70 \%$ & $7,92 \%$ & $10,76 \%$ & $9,56 \%$ \\
\hline
\end{tabular}

Tabla 5.6: Porcentaje de simulaciones que se han producido en una determinada secuencia de fallo por cada grupo de casos de carga

Un dato que hay que destacar es que de los 720 órdenes posibles de rotura sólo sucede alguna vez 46 entre las decenas de miles de simulaciones con todas las variaciones posibles. 
Del grupo de variaciones de todos los parámetros se han escogido las 5 secuencias de rotura que más se repetían 214356, 234156, 243156, 342156 y 432156 y se han formado 6 grupos de trabajo con esas 5 secuencias y otro grupo incluyendo las demás. Y de ahora en adelante se denominaran los grupos 1, 2, 3, 4, 5 y 6 para las secuencias 214356, 234156, 243156, 342156, 432156 y todas las demás, respectivamente. Hay que tener en cuenta que en este caso no se ha considerado indistintamente de uno u otro grupo cuando dos elastómeros rompían al mismo tiempo, por ejemplo si TOP- y SIDE + rompían al mismo tiempo entre el tercero y el cuarto se considera que es del grupo 234156. Siempre del grupo de menor numeración de entre los 6 posibles. En las figuras 5.18, 5.19, 5.20, 5.21, 5.22, 5.23,5.26, 5.27, 5.28, 5.29, 5.30 у 5.31 se muestran los resultados variando los parámetros SIZE, RPM, ANG, STIFF, STR, DAMP, SIZE\&RPM, SIZE\&ANG, SIZE\&STIFF, SIZE\&STR, SIZE\&DAMP y TODO respectivamente. 


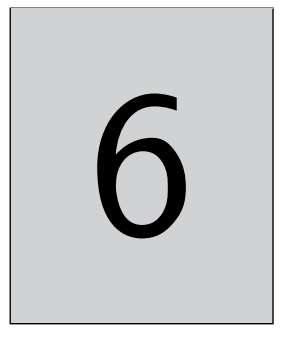

\section{CONCLUSiOnes Y CONSIDERACIONES}

\section{Índice}

6.1. Conclusiones sobre el fenómeno de la pérdida de pala . . . . . . 133

6.1.1. Conclusiones del análisis con variables deterministas . . . . . . . . . . 134

6.1.2. Consideraciones acerca de los resultados deterministas . . . . . . . . . . 135

6.1.3. Conclusiones del análisis con variables aleatorias . . . . . . . . . . . 137

6.1.3.1. Conclusiones del análisis con los primeros resultados preliminares 137

6.1.3.2. Conclusiones de las variaciones de parámetros . . . . . . . . 138

6.1.3.3. Conclusiones de la secuencia de fallo . . . . . . . . . . . . . 139

6.1.4. Consideraciones acerca de los resultados aleatorios . . . . . . . . . . . . 140

6.2. Conclusiones generales . . . . . . . . . . . . . . 142

6.3. Consideraciones generales ................... 143

6.4. Otras consideraciones. Propuestas de futuros trabajos . . . . . 143

En este capítulo se van a mostrar primero en 6.1 las conclusiones derivadas de los resultados obtenidos tanto en el capítulo 4 como en el capítulo 5. A continuación están expuestas en 6.2 las conclusiones generales deducidas a lo largo de todo el trabajo. Finalmente están planteadas en 6.4 una serie de líneas de trabajos posibles a raíz de lo planteado en este estudio.

\subsection{Conclusiones sobre el fenómeno de la pérdida de pala}

En este apartado se muestran las conclusiones derivadas de los capítulos anteriores. Primero se muestran las conclusiones del análisis determinista en 6.1.1 desde el punto de vista del fenómeno en sí, describiendo cómo reacciona el sistema a las cargas. Se muestran también datos obtenidos tanto generales como particulares de entre los grupos de casos de carga analizados. Para acabar con las conclusiones de este método de análisis se valora el propio método como herramienta para este tipo de fenómeno en 6.1.2. En el siguiente sub-apartado 6.1.3 se realiza lo anteriormente mencionado pero para el caso del análisis estadístico con variable aleatoria. Por último se valora el propio método como herramienta para este tipo de fenómeno en 6.1.4. 


\subsubsection{Conclusiones del análisis con variables deterministas}

Inicialmente el modelo empieza a absorber y acumular energía como energía cinética. Las fuerzas entre el motor y la bancada se incrementan hasta que se cumplen los criterios de fallo. La simulación proporciona la secuencia probable de rotura, como se puede ver en la figura 4.16; el apoyo trasero $\mathrm{Y}+$ siempre rompe el primero (antes de $0.2 \mathrm{~s}$ ) seguido del apoyo trasero Y- en un período muy breve de tiempo. Sólo aparece un caso, cuando la rigidez de la unión bancada-ala es extremadamente baja, en el que la secuencia es al revés. Como resultado del fallo, la dinámica del sistema cambia así como los modos propios del sistema. El motor empieza a vibrar de modo distinto, la amplitud del movimiento se incrementa notablemente, y la energía del sistema crece también. Las fuerzas que llegan al ala alcanzan valores considerables y los elementos de la bancada absorben energía interna mediante deformaciones plásticas. Al modelo se le ha proporcionado de una ley de material perfectamente plástica para los elementos 1D y 2D de la bancada. La conclusión en este aspecto es que la plasticidad se debe tener en cuenta debido al número de elementos que alcanzan altos niveles de esfuerzo. Sin embargo, también existen casos de carga en los que no se dan estos niveles tan altos de esfuerzos: cuando el tamaño de pala perdida es menor y la rigidez de la unión bancada-ala es menor.

Se ha encontrado que la frecuencia de la hélice es un parámetro influyente ya que los resultados cambian notablemente con él. Esto es debido al acercamiento de la frecuencia de la hélice con el modo propio más cercano. Se ha encontrado que la energía del modelo es dependiente del caso de carga aplicado, y también sucede con los apoyos delanteros (soft mounts), que fallan cuando los niveles de energía del modelo alcanzan ciertos valores como indicaba la tabla 4.4. Después de los apoyos traseros, el orden típico de fallo es los 'top mounts' (normalmente $\mathrm{Y}+$ en tercer lugar e Y- en cuarto), y después el 'soft mount Y- seguido del Y+. Sin embargo, este orden puede cambiar fácilmente ya que sus admisibles son similares y la fuerza de los apoyos delanteros se incrementa de manera parecida. Aparte de esto, la secuencia de fallo está identificada (ver figura 4.16) para la mayoría de los casos de carga.

El modelo es capaz de detectar zonas con daño en la bancada (ver figura 4.20) y también roturas en los elastómeros (ver figura 4.23). Además, se estudian los parámetros que pueden ser fuentes del fallo potenciales, por ejemplo ciertos ángulos (de la pala en el plano YZ) que se han detectado como ángulos más propensos al fallo; ver la figura 4.21, en la mitad de los casos de carga los apoyos trasero Y+ e Y- fallan cuando el ángulo de la pala de la hélice dañada se encuentra entre $340^{\circ}-345^{\circ}$ y $351^{\circ}-5^{\circ}$ respectivamente. Por lo tanto, el fenómeno de la pérdida de pala se ha estudiado en términos de cuándo, dónde y en qué orden se va a producir el fallo de la unión.

Numerosas conclusiones se han encontrado en los distintos grupos de casos de carga analizados: 
- Las fuerzas en las condiciones de contorno disminuyen cuando el tamaño de pala perdida también lo hace. Lo mismo sucede con el número de elementos plastificados y el nivel de energía del modelo.

- Las fuerzas en las condiciones de contorno disminuyen cuando la rigidez de la unión bancada-ala también lo hace. Lo mismo sucede con el número de elementos plastificados y el nivel de energía del modelo.

- La transmisibilidad depende de la velocidad de rotación de la hélice. El número de elementos plastificados se incrementa cuanto mayor sea el amortiguamiento estructural.

- Los resultados de la simulación se muestran muy influidos por el tamaño de pala que se pierde. También son dependientes del tiempo en el que se introduce la perturbación en el sistema; la evolución de los distintos casos de carga difiere considerablemente. Los resultados de la simulación también dependen de la forma en la que se introduce la perturbación, de la rigidez de la unión bancada-ala, y muy dependiente de la condición de vuelo en la que se produce la perturbación, del tamaño de pala perdida y de la velocidad de rotación de la hélice. En cambio, no hay diferencias significativas cuando se varía el amortiguamiento estructural (en el rango estudiado) o la rigidez del ala.

Por lo tanto, hay parámetros que se han mostrado más influyentes en los que los diseñadores deben fijarse cuando estudian este fenómeno. Algunos de ellos escapan a su control: el tamaño, el ángulo o la frecuencia de la hélice cuando se pierde la pala. Estos resultados son fundamentales para entender el fenómeno, y críticos para diseñar la estructura. Los apoyos se han diseñado para minimizar los riesgos para el resto de la aeronave, fallan en cuanto el motor alcanza altos niveles de energía; los apoyos traseros (rear mounts) se rompen cuando la energía es de 50kJ, los otros apoyos (top and side mounts) cuando la energía es de 400kJ, y esto ocurre en menos de 1 segundo en la mayoría de los casos de carga. Es más, se ha identificado que la rigidez de la unión bancada-ala debe ser muy baja para que disminuya el nivel de energía que llega al resto de la aeronave. La elección de estos dispositivos puede hacer disminuir los riesgos de fallo en el resto de la estructura y puede mejorar las posibilidades de que se pueda llevar a cabo el resto del vuelo.

\subsubsection{Consideraciones acerca de los resultados deterministas}

En lo que respecta a la validez de los resultados obtenidos, el modelo es capaz de simular la secuencia de la pérdida de pala sin inestabilidades y hasta el final del fenómeno para la mayoría de los casos de carga. La secuencia de la simulación se muestra en la figura 6.1, para este caso de carga los apoyos traseros están rotos en el número 4 y los apoyos delanteros lo están también en el número 6. Los chequeos de masa y energía cumplen con los requisitos típicos como se puede ver en 4.4.4.1. 

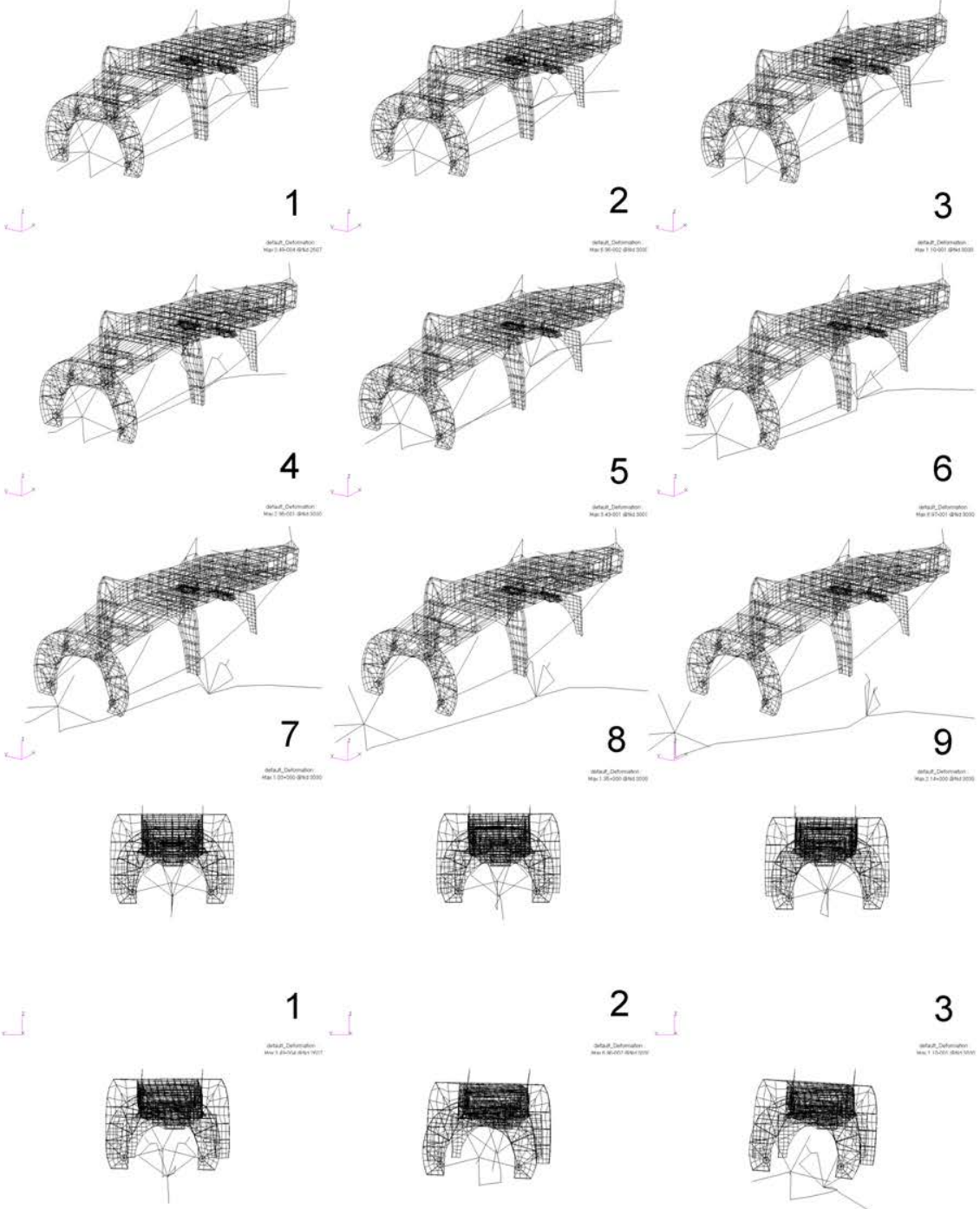

4

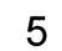

6
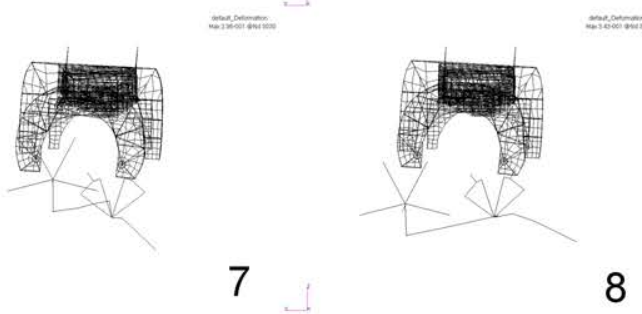

8

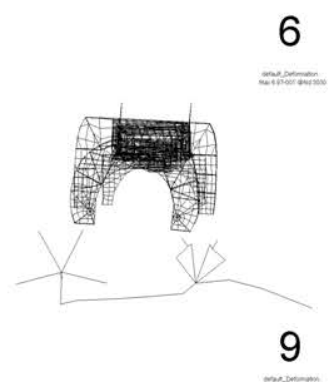

Figura 6.1: Secuencias de la simulación

El modelo se comporta adecuadamente y no aparece un nivel importante de energía de hourglass. La optimización del tamaño del paso del tiempo es crucial para obtener unos buenos resultados con las capacidades computacionales existentes. Las limitaciones debidas al tiempo no han sido una desventaja o inconveniente para analizar la secuencia de rotura. El modelo 
incluye un comportamiento no lineal, y la secuencia de rotura de los "muelles"de los apoyos. El modelo elastomérico es capaz de reproducir la secuencia de fallo.

Cuando alguno de los apoyos falla, los modos propios de la estructura cambian y lo mismo sucede con el comportamiento dinámico del sistema. Por lo tanto, es difícil comparar entre casos de carga debido a las diferencias de tiempos en las que se producen las roturas elastoméricas y, en consecuencia, las distintas secuencias de fallo. Además, la simulación es un análisis explícito dependiente del tiempo y, por lo tanto, pequeños cambios en las primeras etapas de la simulación pueden derivar en grandes diferencias a lo largo de la simulación.

El fenómeno se ha simulado utilizando técnicas explícitas del método de elementos finitos. El código explícito utilizado puede considerarse adecuado para analizar la secuencia de fallo y el comportamiento dinámico directamente relacionado con la pérdida de la pala.

Finalmente, se puede añadir que los resultados que se quieran obtener de simulaciones de larga duración deben estudiarse mediante códigos implícitos (aunque se han mantenido los errores del modelo en niveles muy bajos) y/o realizar un número suficiente de simulaciones con los que se puedan obtener conclusiones cuantitativas. En caso contrario los resultados del análisis explícito en estos casos son cualitativos, cabe destacar que solamente se han podido estudiar los resultados de 45 simulaciones y en cada grupo de casos de carga entre 3 y 10 simulaciones cada uno. Esto implica que hay tendencias que en cuanto no son seguidas por todas las simulaciones de la misma manera no se pueden sacar conclusiones representativas. Ha resultado difícil obtener resultados concluyentes basándose en un número de simulaciones pequeño, sobre todo cuando la tendencia a determinar no es clara.

\subsubsection{Conclusiones del análisis con variables aleatorias}

En este caso el sub-apartado se subdivide primero en los primeros resultados que se obtuvieron con el modelo sólo motor para luego ya comentar las conclusiones tanto de cada parámetro y combinaciones de ellos como de la secuencia del fallo.

\subsubsection{Conclusiones del análisis con los primeros resultados preliminares}

Los resultados preliminares de 5.4.1, que fueron los primeros que se obtuvieron considerando sólo el motor, permiten deducir conclusiones muy relevantes de los resultados obtenidos. Por ejemplo, el tiempo entre la primera rotura de la unión y la última es casi invariable cuando el tamaño de la pala perdida está en los siguientes valores; es $\sim 0.078 \mathrm{~s}$ cuando el ratio de la pala perdida frente a la pala completa es de entre 0 y 0,65 y $\sim 0,066$ s cuando el ratio de la pala perdida frente a la pala completa es de entre 0,65 y 0,75 . En el caso de que los otros parámetros también influyan en la simulación, el tiempo entre la primera rotura de la unión y la última es en más del $80 \%$ de los casos entre 0.055 y 0.085 s. Esto se puede ver en la figura 6.2. 


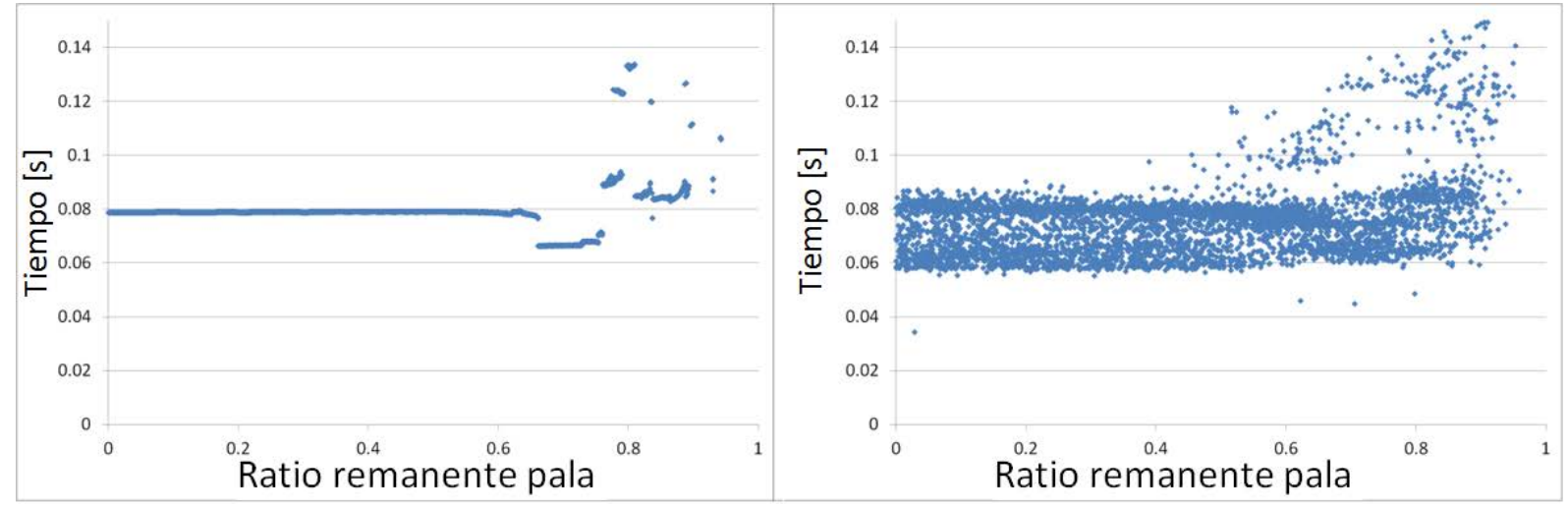

Figura 6.2: Tiempo entre la primera y la última rotura vs. sólo la variación del ratio de pala perdida (izquierda) y con todas las variaciones de entrada (derecha)

También cabe destacar que la decisión tomada en el capítulo 5 acerca del amortiguamiento estructural resulta más acertada que en caso del capítulo 4. En el capítulo 4 se amortiguó el sistema según la primera frecuencia natural del sistema, sin embargo, las fuerzas exteriores no excitan directamente esta frecuencia por lo que los resultados obtenidos no son tan provechosos como en el caso del capítulo 5 en el que se amortiguó el sistema a la frecuencia de la hélice.

\subsubsection{Conclusiones de las variaciones de parámetros}

El análisis muestra que el impacto sobre los resultados cambia dependiendo de qué parámetro sea el que se ha variado; la desviación típica de la tabla 5.4 muestra el orden de magnitud de la influencia. La evolución del análisis cuando se varía el tamaño de pala perdida se dispersa mucho más que cuando se varía la rigidez de los elastómeros o la frecuencia de la hélice cuando se rompe la pala. Los parámetros resistencia de los elastómeros, amortiguamiento estructural y posición angular de la pala cuando se separa el motor se encuentran en nivel medio de influencia en los resultados.

El set de análisis completo incluye más que variaciones individuales de parámetros, como se puede ver en el apartado 5.3.2. Como ejemplo está la figura 5.11 que muestra la variación de SIZE\&ANG. Debido a la gran influencia individual de SIZE los resultados son muy similares a esta variación (ver la figura 5.10) con unos efectos de segundo orden que dispersan ligeramente los resultados. Por ejemplo, en el caso del modelo E+EMS cuando el valor de SIZE es cero PAF vale alrededor de $170^{\circ}$, FFT $t=0,028 \mathrm{~s}$ y FLT alrededor de $t=0,07 \mathrm{~s}$, y para el caso de E PAF vale alrededor de $10^{\circ}$, FFT $t=0,023 \mathrm{~s}$ y FLT alrededor de $t=0,09 \mathrm{~s}$.

Finalmente, se ha llevado a cabo un análisis entre todos los parámetros de entrada, la matriz de correlación se puede ver en la figura 5.12 y se han obtenido conclusiones de los resultados. El tiempo entre la primera y última rotura de elastómero es constante para varios rangos de tamaño de pala (ver SIZE vs. FLT en la figura 6.3). El valor es de $\sim 0,04 s$ cuando 
el ratio de pala perdida está entre $0-0,37$ y $0,67-0,84, \sim 0,06 s$ cuando el ratio de pala perdida está entre 0,37-0,67 y se incrementa cuando el ratio de pala perdida es $>0,84$. Incluso teniendo en consideración todas las variaciones de los parámetros de entrada, el tiempo entre la primera y última rotura de los apoyos (FLT) es en más del $75 \%$ de los casos de carga entre $0,035 s$ y $0,07 s$. En lo que respecta a las variables PAF y PAL no hay ángulos que se hayan mostrado más propensos a que se produzca una rotura, como se puede ver en la figura 5.17 y en la última columna de la tabla 5.4. Los resultados muestran de una manera clara patrones de
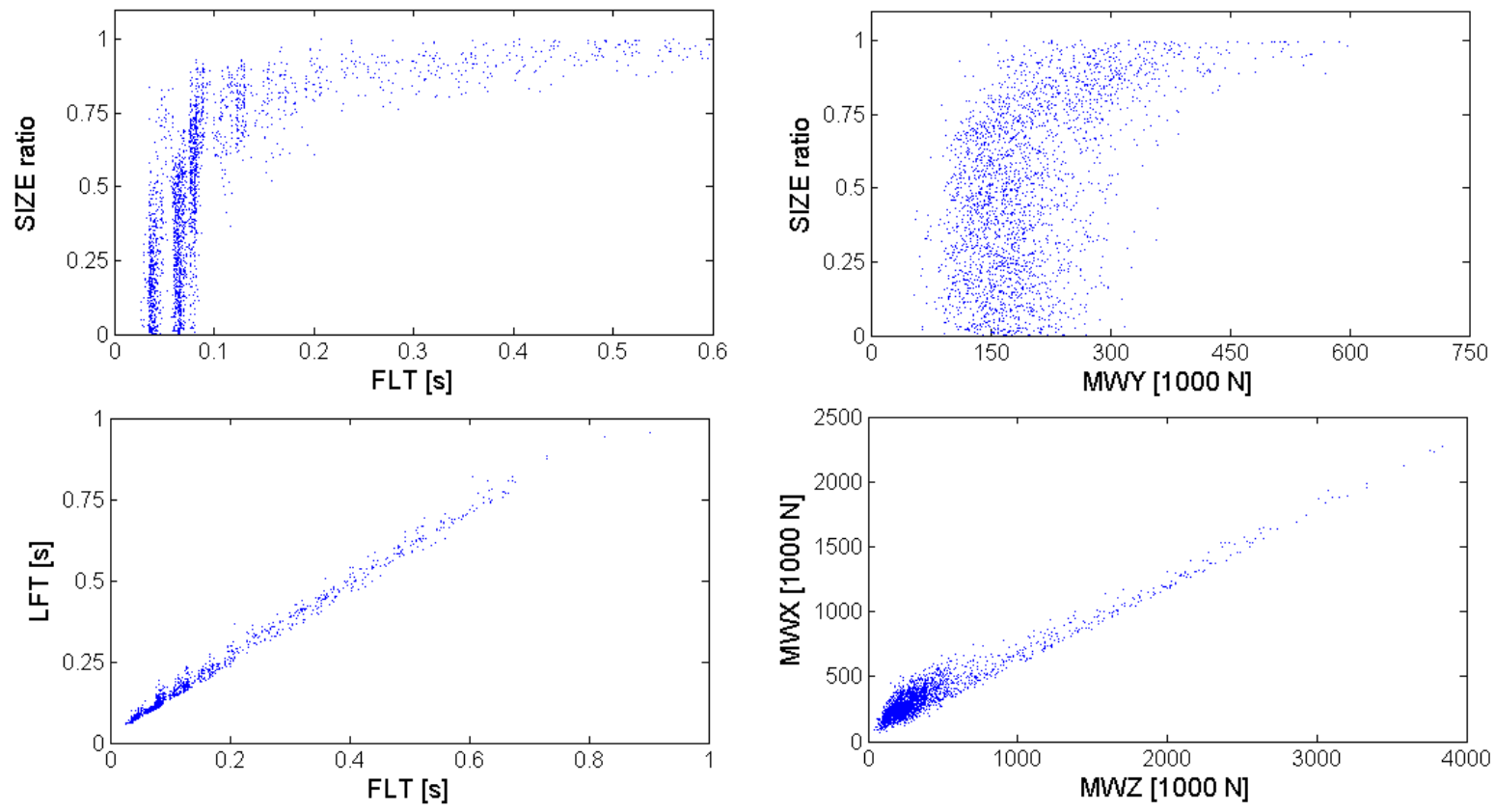

Figura 6.3: Detalle de los resultados con variación de todos los parámetros de entrada comportamiento en algunas variables, principalmente entre la variable SIZE y todas las demás, y entre variables de salida. Por ejemplo (ver figura 6.3) SIZE vs. MWY muestra un resultado curioso, cuando MWY se incrementa SIZE se incrementa en media. El gráfico LFT vs. FLT muestra una gran correlación entre ambas variables, y lo mismo sucede con MWX vs. MWZ.

\subsubsection{Conclusiones de la secuencia de fallo}

De los resultados mostrados en 5.4.5 se puede deducir que en casi todas ellas se observan zonas con la misma secuencia de fallo y se pueden obtener otra serie de conclusiones como son:

- En la variación de SIZE: las secuencias de fallo 4 y 5 son las más probables (ver la tabla 5.6), la secuencia 4 de fallo se produce para ratios de pala perdida extremas $>0.75$ o $<0.3$. Lo mismo sucede con la secuencia de fallo 2 sólo se produce para ratios $>0.5$ (ver la figura $6.4)$. 
- En la variación de RPM: no suceden las secuencias 1, 2 y 3, la secuencia 4 de fallo solamente se produce entre 75-80 [rad/s] y la secuencia 5 justo entre 73-75 [rad/s] (ver la figura 6.4). Aun teniendo frecuencias superiores e inferiores la secuencia 4 es la que produce una mayor fuerza en los apoyos según el eje Y. También en el eje X pero el máximo ya no es exclusivo sólo de esa secuencia.

- En la variación de ANG: no se pueden sacar conclusiones fácilmente. Se observa que la secuencia 2 es una de las secuencias en las que se produce el fallo del último elastómero más tarde (LFT) y también en la que el tiempo entre el primer y último fallo de elastómero es mayor (FLT) (ver la figura 6.4).

- En la variación de STIFF: las secuencias de fallo 4 y 5 son las más probables, en la secuencia 2 y 5 siempre rompe el primer elastómero lo antes posible, al contrario que en la mayoría de las veces de la secuencia 4 (ver la figura 6.4).

- En la variación de STR: de las secuencias estudiadas sólo se produce la secuencia 4 y 5 (ver la tabla 5.6) y para valores de STR entre 1 y 1.1 pero ésta se encuentra en valores medios de los parámetros de salida (ver la figura 6.4).

- En la variación de DAMP: de las secuencias estudiadas las secuencias de fallo 4 y 5 son las más probables (ver la tabla 5.6), es más, sólo se producen la secuencia 2, 4 y 5 . La secuencia 2 y 5 son unas secuencias que se producen el fallo del último elastómero antes (LFT) y también en la que el tiempo entre el primer y último fallo de elastómero es menor (FLT). También la secuencia 2 es la que produce menores fuerzas en los apoyos (MWX, MWY y MWZ).

En el caso de variaciones de dos parámetros se encuentran resultados muy diferentes. En el caso de la variación de SIZE\&ANG no se sacan conclusiones adicionales aparte de las mencionadas en las variaciones individuales. Sin embargo en el caso de la variación de SIZE\&RPM, SIZE\&STIFF, SIZE\&STR y SIZE\&DAMP se ven patrones clarísimos de secuencias de fallo según sean las variables (ver la figura 6.5). Las secuencias se encuentran más o menos por las mismas zonas dentro de la variable SIZE ya que como se ha mencionado antes es el parámetro más influyente sin embargo según sea la variación del otro parámetro los patrones cambian y se modifican.

\subsubsection{Consideraciones acerca de los resultados aleatorios}

En lo que respecta a la validez de los resultados obtenidos, el modelo es adecuado para la simulación de la secuencia de fallo y es capaz de converger sin inestabilidades hasta el final del fenómeno para todos los casos de carga. El modelo se comporta de manera eficiente y la evolución de la energía se ha chequeado. La optimización del paso del tiempo y las simplificaciones llevadas a cabo en el modelo FEM son cruciales para conseguir la solución más adecuada con los recursos computacionales disponibles. 

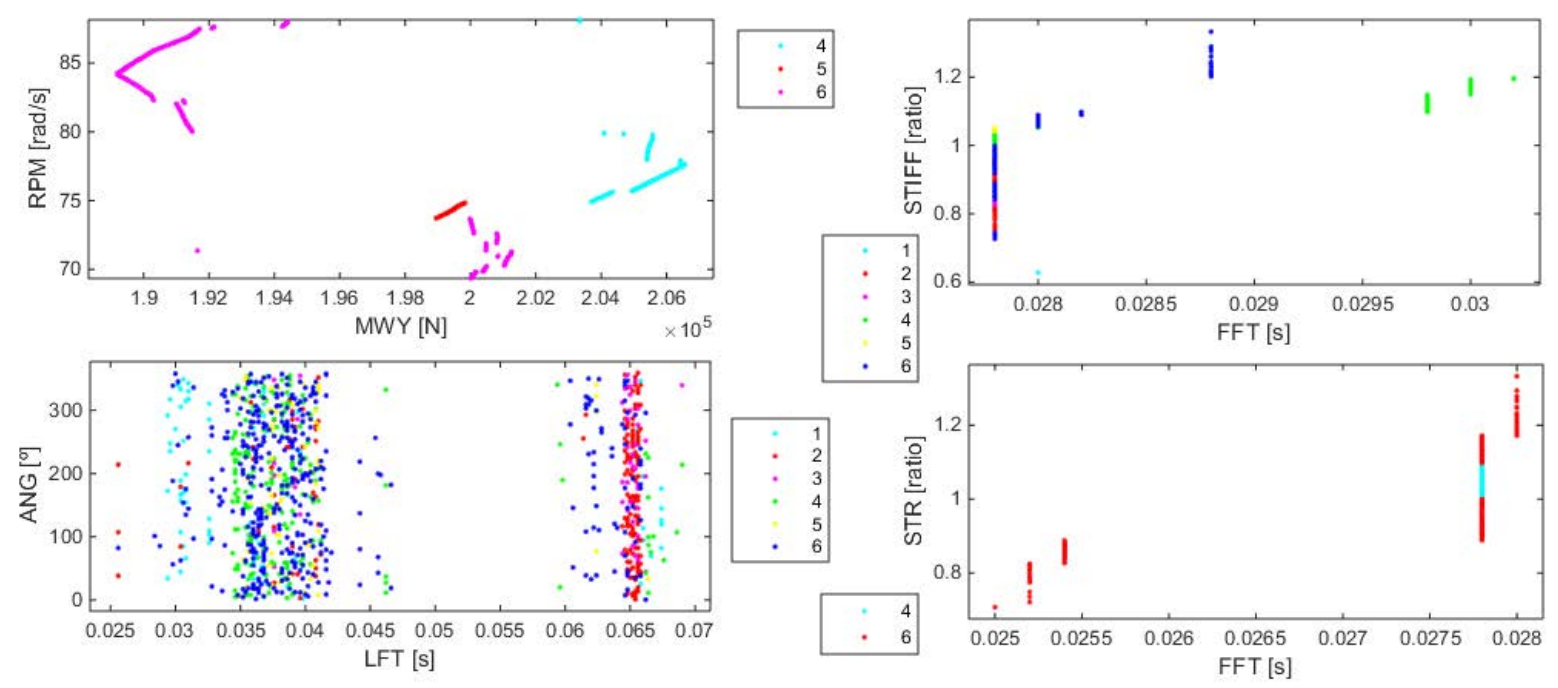

Figura 6.4: Resultados variando un parámetro de entrada en función de uno de salida identificando con colores las secuencias de fallo
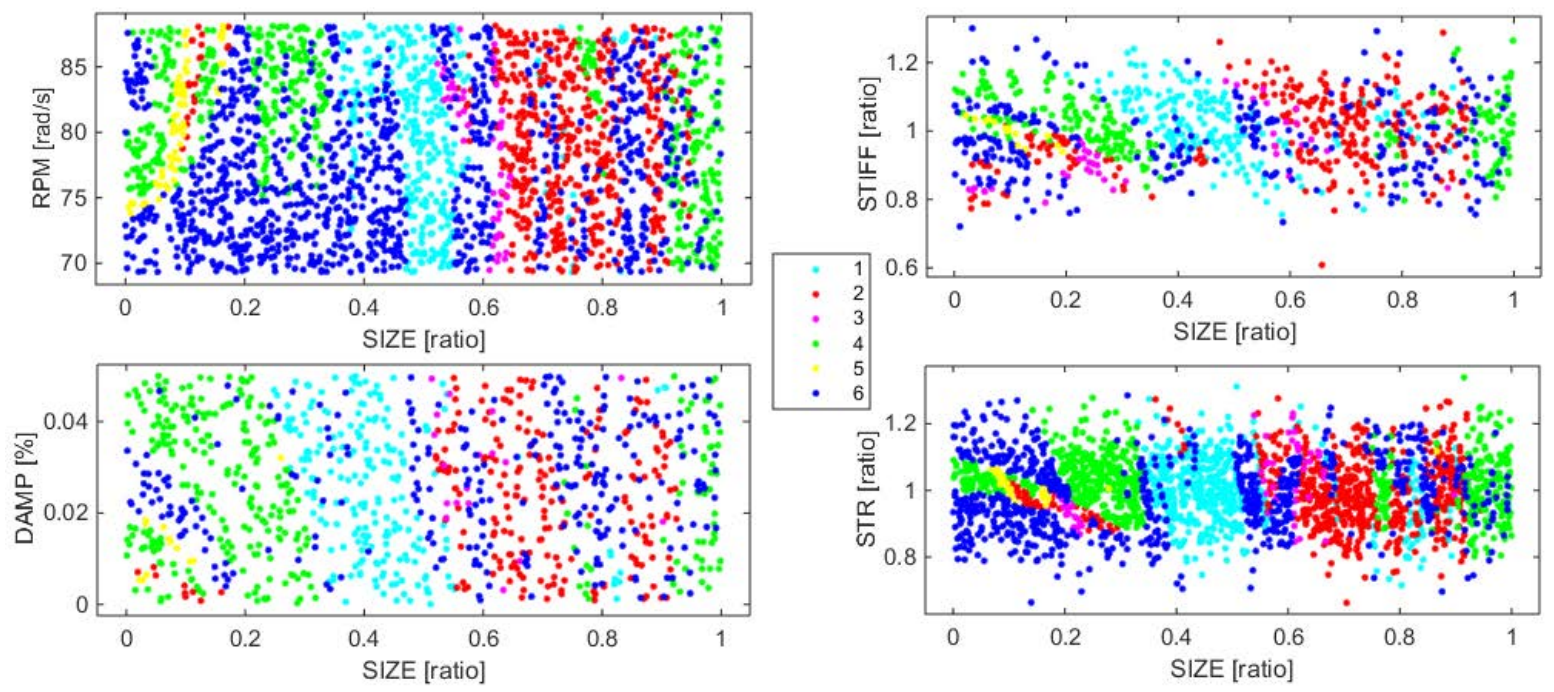

Figura 6.5: Secuencias de fallos (colores) en función de la variación de dos parámetro de entrada

El modelo incluye comportamiento no lineal y rotura mediante criterios de fallo. El modelo elastomérico es capaz de reproducir la secuencia de fallo aun cuando los apoyos se van rompiendo que implica cambios en los modos propios y, en consecuencia, de la dinámica del sistema.

Asimismo, los análisis se han realizado utilizando distintas estrategias, distintos esquemas y métodos estadísticos (técnica de Monte Carlo). Esta técnica es capaz de conseguir resultados interesantes; distintas secuencias de fallo dependiendo de los parámetros iniciales, cuánto tiempo se tarda en que el motor se separe, y cuáles son las consecuencias para el ala durante este tiempo. Asimismo, los apoyos delanteros top y side suelen romper los primeros $(\sim 0,045 s$ de media), después los traseros (el intervalo de tiempo entre la primera y última rotura es de 
$0,035 s-0,07 s)$ y el motor queda libre. Además, el fenómeno de la pérdida probablemente daña el ala en dirección Y para los límites admisibles escogidos para la unión bancada-ala, esto es debido a que no está diseñado para absorber este tipo de carga para las condiciones normales de vuelo. Se ha hecho un estudio para resolver esta incidencia y determinar los admisibles necesarios para que estos apoyos soportaran el fenómeno con una probabilidad de fallo baja.

Por lo tanto, el análisis identifica los parámetros más importantes que pueden hacer cambiar peligrosamente el comportamiento de la estructura. Los resultados dependen notablemente del tamaño de pala perdida, y en un segundo orden de influencia de la resistencia de los elastómeros, el amortiguamiento estructural, la frecuencia de la hélice y la posición angular de la pala perdida, y casi no depende de la rigidez de los elastómeros para el rango de valores escogido. El modelo es capaz de estudiar los rangos de los parámetros en los que la simulación progresa de la misma manera. En consecuencia, se identifican qué parámetros y por qué son los responsables de diferencias significativas en los resultados cuando hay sólo pequeñas diferencias en las variables de entrada.

Además, cabe destacar que al contrario del caso determinista (45 simulaciones) en este caso sí que se han realizado un número suficiente de simulaciones (como mínimo 1000 hasta 10000) como para determinar las conclusiones obtenidas. Se ha señalado la enorme cantidad de información que hay en la matriz de resultados variando todos los parámetros que permite postprocesar la variación de cualquier combinación de parámetros de entrada y/o salida en función de cualquier combinación de parámetros de entrada y/o salida. Gracias a esto, se han podido determinar con fiabilidad las conclusiones obtenidas.

\subsection{Conclusiones generales}

En estos capítulos se han mostrado diferentes estrategias para estudiar el análisis de fallos dinámicos. Los ejemplos de investigación cubren una amplia gama de los fenómenos dinámicos. Se han incluido desde ejemplos muy sencillos para introducir las herramientas de análisis como el ejemplo 2.3.2 resuelto con distintas técnicas, el ejemplo 3.1.1 para introducir la metodología para incluir los contactos y choques y el ejemplo 3.3.2 con una viga con carga armónica aplicada a la que posteriormente se le añadieron variables aleatorias en el ejemplo 5.1.1. El resto de ejemplos de casos estudiados son sucesos muy dinámicos, que contienen comportamientos no lineales, daños y fallos. Uno de estos casos estudiados fue un impacto a baja velocidad en 3.1.2, que como se indicó puede producirse de muchas maneras, tales como una caída de herramienta, granizo y restos en la pista de aterrizaje. Uno de las propiedades más importantes que se estudian es la resistencia residual de la estructura y, por lo tanto, su tolerancia a los daños. Estos ejemplos permitieron el estudio del impacto en sí mismo, siendo capaces de predecir la respuesta y la extensión de la zona delaminada. Además se ha presentado un estudio frangibilidad en 3.2 donde la estructura tiene que romperse fácilmente bajo ciertas condiciones; sin embargo, tiene que soportar otras 
cargas sin fallo alguno. Éste es un concepto con el que los ingenieros no están muy familiarizados y con el que no trabajan habitualmente. En el estudio se aplican los diferentes casos de carga y simula el fenómeno con precisión y permite obtener valiosas conclusiones. Finalmente, el último caso de estudio muestra la simulación de un posible incidente en vuelo, la pérdida de pala de un avión turbohélice cuyas conclusiones se muestran en detalle en el apartado 6.1.3. Los análisis llevados a cabo se realizaron con distintas estrategias, metodologías deterministas y estadísticas (técnica de Monte Carlo). Como se ha mostrado cada estrategia es capaz de lograr resultados útiles en mayor y menor medida.

\subsection{Consideraciones generales}

Se puede considerar que en este estudio se han analizado las diferentes formulaciones existentes cuando se estudian diferentes parámetros en problemas estructurales con cargas y/o propiedades, tanto deterministas como aleatorias, altamente dinámicas y no-lineales. Sobre todo, se ha enfocado a estructuras aeronáuticas, con sus elementos estructurales típicos (vigas y placas) y las propiedades y cargas a las que están sometidas.

Se ha reflexionado sobre la forma de analizar problemas dinámicos muy complejos. Se han evaluado los resultados de los métodos deterministas con el método de elementos finitos que son los más extendidos en el campo del cálculo estructural en busca de sus limitaciones y se han propuesto alternativas. Se han resuelto las ecuaciones de los sucesos tanto con esquemas tanto explícitos como implícitos. Se ha buscado con cada problema las mejores opciones de modelización para la resolución del problema. Se ha conseguido mejorar el conocimiento de la respuesta de los parámetros de interés con las herramientas que se han planteado. También se han desarrollado modelos optimizados sin penalización en los mismos para conseguir la aproximación a problemas estructurales difícilmente abordables en la actualidad.

Finalmente se atacó el problema con variables aleatorias debido a que existen incertidumbres o propiedades que no pueden reproducirse adecuadamente con variables deterministas. En la modelización se ha discretizado el campo utilizando tanto variables gaussianas como nogaussianas ya que las variables o se desconocían, tenían incertidumbres o incluso podían valer cualquier valor dentro de un rango.

\subsection{Otras consideraciones. Propuestas de futuros trabajos}

En el abordaje del estudio de estas líneas de investigación han surgido distintos fenómenos en los que podría realizarse un estudio con mayor profundidad como es la teoría de los valores máximos. A estos fenómenos se les podría aplicar tanto la técnica Monte Carlo como aquí se ha realizado como resolver desde un punto de vista analítico. En este caso tienen que ser sucesos en los que aun siendo dinámicos se pueda representar por ecuaciones analíticas ya que la dis- 
tribución de alcance de valores máximos puede complicarse si se tiene en cuenta una serie de variables aleatorias dependientes entre ellas. Esto es debido al hecho de que se debe calcular la distribución conjunta de la serie de variables aleatorias. Cabe destacar que contrariamente al caso de una única variable independiente en donde existe una única distribución en los problemas las distribuciones muchas veces son dependientes entre ellas. En algunos casos que se han planteado a lo largo del trabajo y dependiendo de la estructura se ha determinado que bajo ciertas condiciones las distribuciones son idénticas. Sin embargo, habría que continuar en esta línea de investigación ya que una pequeña dependencia aparentemente despreciable puede derivar en grandes diferencias en los modelos de valores máximos por lo que hay que tener precaución al realizar estos análisis.

De una manera similar, se puede atacar el problema con el concepto de valor umbral como se ha realizado aquí con las fuerzas de las condiciones de contorno o las veces en las que una función sobrepasa cierto umbral. Igualmente se podría añadir además la condición temporal de que tal circunstancia ocurra antes de cierto tiempo.

Otro de los problemas importantes al que nos hemos enfrentado es la incertidumbre en las variables. Estas incertidumbres pueden tener distintas fuentes; pueden ser incertidumbres inherentes o físicas, incertidumbres del modelo,... La identificación de las fuentes es otro de los trabajos fundamentales ya que la veracidad de los resultados van a depender de la correcta identificación de las fuentes. Y esto puede ocurrir tanto en propiedades estructurales como en las características de la carga. Estas incertidumbres pueden ser debidas al desconocimiento del comportamiento estructural o a las simplificaciones introducidas por los modelos. En el caso de incertidumbres en la estimación de los errores se debe reducir mediante la correcta aplicación del modelo estadístico. Por estos motivos y como se ha comentado es importante reducirlas lo máximo posible conociendo y estudiando el fenómeno físico del problema. Con las herramientas planteadas en la tesis se puede tratar de estudiar otros fenómenos con incertidumbres en los parámetros. Por ejemplo, en el modelo matemático esto puede afectar tanto a los modelos de las leyes de material (linealidad, isotropía, homogeneidad) como a la relación esfuerzo-deformación o los modelos de amortiguación que se han estudiado en este trabajo además de otras variables. 


\section{REFERENCIAS}

P. R. Adduri. Robust Estimation Of Reliability In The Presence Of Multiple Failure Modes. $\mathrm{PhD}$ thesis, Wright State University. B. E., Andhra University, M.E., Lamar University., India, 2006.

S. Adhikar. Joint distribution of eigenvalues of linear stochastic systems. Austin, Texas, 46th AIAA/ASME/ASCE/AHS/ASC Structures, Structural Dynamics and Materials Conference. 18 - 21 April 2005, Austin, Texas, 42005.

S. Adhikar. An unified parametric-nonparametric uncertainty quantification approach for linear dynamical systems. Waikiki, Hawaii, 48th AIAA/ASME/ASCE/AHS/ASC Structures, Structural Dynamics and Materials Conference., 4 2007a.

S. Adhikar. Uncertainty propagation in linear systems. an exact solution using random matrix theory. Honolulu, Hawaii, 48th AIAA/ASME/ASCE/AHS/ASC Structures, Structural Dynamics, and Materials Conference., 4 2007b.

S. Adhikar. Response variability of linear stochastic systems. a general solution using random matrix theory. Schaumburg, IL, 49th AIAA/ASME/ASCE/AHS/ASC Structures, Structural Dynamics and Materials Conference., 42008.

S. Adhikar y M. I. Friswell. Random eigenvalue problems in structural dynamics. Palm Springs, California, 45th AIAA/ASME/ASCE/AHS/ASC Structures, Structural Dynamics and Materials Conference., 42004.

S. Adhikar y Phani A. S. Random eigenvalue problems in structural dynamics: Experimental investigations. AIAA Journal, 48(6), 2010.

Federal Aviation Administration. Turbine engine rotor blade containment-durability. Advisory Circular AC 33.5, Federal Aviation Administration. U.S. Department of Transportation., Washington, DC., 1990. 
Federal Aviation Administration. Guidance material for fatigue limit tests and composite blade fatigue substantiation. Advisory Circular AC 35.37-1A, Federal Aviation Administration. U.S. Department of Transportation, Washington, DC., 2001.

ASN. Asn aviation safety database » airplane - engines. Technical report, Flight Safety Foundation, Última actualización: 12 de abril de 2015, accedido el 12 de abril de 2015. URL http://aviation-safety. net/database/events/event . php?code=AC.

M. Bah, P. B. Nair, A. Bhaskar, y A. J. Keane. Stochastic component mode synthesis. Norfolk, Virginia, 44th AIAA/ASME/ASCE/AHS Structures, Structural Dynamics, and Materials Confere, 42003.

A. T. Beck y E. da Rosa. Structural reliability analysis using deterministic finite element programs. Latin American Journal of Solids and Structures., 3:197-222, 2006.

F. Bertagnolio, F. Rasmussen, N. Sørensen, J. Johansen, y H. Aa. Madsen. A stochastic static stall model applied to a wind turbine blade. Honolulu, Hawaii, 26th AIAA Applied Aerodynamics Conference., 82008.

K. Billah y R. Scanlan. Resonance, tacoma narrows bridge failure, and undergraduate physics textbooks. American Journal of Physics, 59(2):118-124, 1991. doi: 10.1119/1.16590.

Y. Bin. Blade containment evaluation of civil aircraft engines. Chinese Journal of Aeronautics, 26:9-16, 2012.

Y. Bin y Y. Zhou. Icing certification of civil aircraft engines. Procedia Engineering, 17:603-615, 2011.

National Transportation Safety Board. In-flight loss of propeller blade and uncontrolled collision with terrain. 1993. Mitsubishi MU-2B-60, N86SD. April 19,1993.

M. C. Boyce y E. M. Arruda. Constitutive models of rubber elasticity: A review. Rubber Chemistry and Technology, 73:504-523, 2000. Issue 3.

M. A. Cesare y R. H. Sues. Profes probabilisitc finite element system - bringing probabilistic mechanics to the desktop. AIAA, 1607, 1999.

James W. Cooley y John W. Tukey. An aigorithm for the machine calculation of complex fourier series. 19(90):297-301, 1965.

R. R. Craig y M. C. C. Bampton. Coupling of substructures for dynamic analyses. AIAA Journal, 6(7):1313-1319, 1968.

S.H. Crandall y W.D. Mark. Random Vibration in Mechanical Systems. Academic Press, New York and London, 2 edition, 1963. ISBN 63-15992. 
A. Der Kiureghian. Analysis of structural reliability under parameter uncertainties. Probabilistic Engineering Mechanics, 23(4):351-358, 2008.

J. M. Dhainaut, J. Cheng, y C. Mei. Nonlinear response of plates under uniform random loads unsynchronized in time. Honolulu, Hawaii, 48th AIAA/ASME/ASCE/AHS/ASC Structures, Structural Dynamics, and Materials Conference., 42007.

H. Diken y K. Alnefaie. Effect of unbalanced rotor whirl on blade vibrations. Sound and Vibration, 330:3498-3506, 2011.

O. Ditlevsen y H.O. Madsen. Structural reliability methods. John Wiley and Sons, 2.3.7 edition, 2007. ISBN 0471960861.

Z. Dygadlo, Kowaleczko G., y W. Sobieraj. The effect of some kinds of blade failures on a helicopter flight dynamics. Number AIAA 2003-208, Reno, Nevada, January 2003. doi: 10. 2514/6.2003-208.

D.J. Elder, R.S. Thomson, M.Q. Nguyena, y M.L. Scott. Review of delamination predictive methods for low speed impact of composite laminates. Composite Structures, 66:677-683, 2004.

G.E. Eyre y W. Spottiswoode. Tay bridge disaster. Technical report, Houses of Parlament, 1880.

B. G. Falzon. Impact damage and repair of composite structures. Technical Report GARTEUR TP155, GARTEUR AG28, 2006.

B. Faust. Evaluation Of The Residual Load-Bearing Capacity Of Civil Structures Using Fuzzy-Logic and Decision Analysis. PhD thesis, Universität Der Bundeswehr. Fakultät Für Bauingenieur- Und. Vermessungswesen. Institut Für Mechanik Und Statik., 2003.

L. M. Fitzwater y C. A. Cornell. Using environmental contours to predict extreme events on wind turbines. Reno, Nevada, 41st Aerospace Sciences Meeting and Exhibit., 12003.

L. M. Fitzwater y S. R. Winterstein. Predicting design wind turbine loads from limited data comparing random process. AIAA, 46, 2001.

I. Fraga y C. Neves. International Encyclopedia of Statistical Science. Chapter:Extreme Value Distributions. Springer Berlin Heidelberg, 2010.

R. Ghanem y A. Sarkar. Numerical analysis of stochastic dynamical systems in the mediumfrequency range. Manchester, UK, RTO AVT Symposium., 102001.

R. Ghanem, J. Red-Horse, A. Benjamin, A. Doostan, y Y. Zou. Stochastic process model for material properties under incomplete information. Honolulu, Hawaii, 48th AIAA/ASME/ASCE/AHS/ASC Structures, Structural Dynamics, and Materials Conference., 42007. 
D. Ghosh y R. Ghanem. A new algorithm for solving the random eigenvalue problem using polynomial chaos expansion. Austin, Texas, 46th AIAA/ASME/ASCE/AHS/ASC Structures, Structural Dynamics and Materials Conference., 42005.

D. Ghosh, R. Ghanem, y J. Red-Horse. Analysis of eigenvalues and modal interaction of stochastic systems. AIAA Journal, 43(10), 2005.

R. M. Gray. Probability, Random Processes, and Ergodic Properties. PhD thesis, Information Systems Laboratory, Electrical Engineering Department, Stanford University., SpringerVerlag, New York, 2010.

M. Guida, F. Marulo, M. Meo, y S. Russo. Certification by birdstrike analysis on c27j fullscale ribless composite leading edge. International Journal of Impact Engineering, 54:105-113, April 2013.

S. Gupta. Reliability Analysis of Randomly Vibrating Structures with Parameter Uncertainties. $\mathrm{PhD}$ thesis, Department Of Civil Engineering, Indian Institute Of Science., Bangalore - 560 012, India, 2004.

S. Gupta y van Gelder P.H.A.J.M. Extreme value distributions for nonlinear transformations of vector gaussian processes. Probabilistic Engineering Mechanics, 22(2):136-149, 2007.

K. R. Gurley, A. Kareem, y M. A. Tognarelli. Simulation of a class of non-normal random processes. International journal of non-linear mechanics, 31(5):601-617, 1996.

J. E. Hall, Hammerschmidt J. Francis II, R. T., J. J. Goglia, y G. W. Black. In-flight loss of propeller blade and uncontrolled collision with terrain. 1996. Atlantic SouthEast Airlines, inc., flight 529 Embraer EMB-120RT, N256AS. August 21, 1995.

C. M. Harris y A. G. Piersol. Shock and Vibration Handbook. McGraw-Hill, 5th edition, 2002. ISBN 0-07-137081-1.

J.J. Hickey. Minimizing the hazards from propeller blade and hub failures. (AC 25.905-1), 2000.

D. H. Hoitsma, E. L. Anagnostou, S. J. Engel, J. S. Madsen, y J. M. Papazian. Structural integrity prognosis system reasoning. Orlando, Florida, 51st AIAA/ASME/ASCE/AHS/ASC Structures, Structural Dynamics, and Materials Conference., 42010.

J. Huh y A. Haldar. A novel risk assessment for complex structural systems. IEEE TRANSACTIONS ON RELIABILITY, 60(1), 2011.

L. Huyse y M. P. Enright. Efficient statistical analysis of failure risk in engine rotor disks using importance sampling techniques. Norfolk, Virginia, April 2003. 
R.A. Ibrahim y C.S. Manohar. Progress in structural dynamics with stochastic parameter variations 1987-1998. Applied Mech. Reviews., 52(5):177-197, 1999.

ICAO. Aerodrome design manual. part 6: Frangibility. Technical Report Doc 9157-AN/901 P6, ICAO, 2006.

R. Ishida. Stochastic finite element analysis of beam with statistical uncertainties. $A I A A$ Journal, 39(11), 2001.

JCGM. Evaluation of measurement data - supplement 1 to the guide to the expression of uncertainty in measurement. 101, 2008.

S. Kinnersley y A. Roelen. The contribution of design to accidents. Safety Science, 45:31-60, January-February 2007. Issues 1-2.

L. Kärger, J. Baaran, A. Gunnion, y R. Thomson. Evaluation of impact assessment methodologies. part i: Applied methods and part ii: Experimental validation. Composites Part B, 40: 71-76, 2009.

M. C. Kushan, S. F. Diltemiz, y I. Sackesen. Failure analysis of an aircraft propeller. Engineering Failure Analysis, 14:1693-1700, 2007.

D. J. Laino y K. G. Pierce. Evaluating statistical loads extrapolation methods. AIAA, 64, 2000.

C. Lalanne. Mechanical Vibration and Shock Analysis. Random vibration., volume 3. John Wiley and Sons., 2nd edition, 2009. ISBN 978-1-84821-124-7.

Y. K. Lee y D. S. Hwang. A study on the techniques of estimating the probability of failure. Journal of the chungcheong mathematical society, 21(4), 2008.

Z. Lei y C. Qiu. A stochastic varational formulation for nonlinear dynamic analysis of structure. Comput. Methods Appl. Mech. Engrg., 190:597-608, 2000.

B.J. Leira. Extremes of gaussian and non-gaussian vector processes: a geometric approach. 25: 401-422, 2003.

X. Lin y J. Zong, Z.andNiu. Finite element model validation of bridge based on structural health monitoring part ii: Uncertainty propagation and model validation. Journal of traffic and transportatio engineering, 2(4):279-289, 2015.

Y. Lu. Random Vibration Analysis of Higher-Order Nonlinear Beams and Composite Plates with Applications of ARMA Models. PhD thesis, Faculty of the Virginia Polytechnic Institute and State University, Blacksburg, Virginia, 2008. 
S. Mahadevan y C. C. Chamis. Structural system reliability under multiple failure modes. AIAA, 1379-CP, 1993.

S. Mahadevan y A. Dey. Adaptive monte carlo simulation for time-variant reliability analysis of brittle structures. AIAA Meeting Papers on Disc, 1647:1326-1332, 1996.

S. Mahadevan y A. Dey. Adaptive monte carlo simulation for time-variant reliability analysis of brittle structures. AIAA Journal, 35(2), 1997.

C.S. Manohar y S. Gupta. Modeling and evaluation of structural reliability:current status and future directions. Research reviews in structural engineering, Golden Jubilee Publications of Department of Civil Engineering, 2002.

C.S. Manohar y R. N. lyengar. Probability distribution of the eigenvalues of systems governed by the stochastic wave equation. Probabilistic Engineering Mechanics, 8:57-64, 1993.

S. F. Masri, R. Ghanem, F. Arrate, y J. P. Caffrey. Stochastic nonparametric models of uncertain hysteretic oscillators. AIAA Journal, 44(10), 2006.

G. Maymon. Probability of failure of structures without a closed form failure function. 49(2): 301-303, 1993.

G. Maymon. Some Engineering Applications in Random Vibrations and Random Structures., volume 178. AIAA, 1998. ISBN 1-56347-258-9.

G. Maymon. Probability of structural failure of a satellite launching motor. Journal of Spacecraft and Rockets., 36(1):177-197, 1999.

G. Maymon. Virtual crack propagation tests with application to probability of failure estimation. Norfolk, Virginia, 44th AIAA/ASME/ASCE/AHS Structures, Structural Dynamics, and Materials Confere., 42003.

G. Maymon. What about uncertainties in the model? Austin, Texas, 46th AIAA/ASME/ASCE/AHS/ASC Structures, Structural Dynamics and Materials Conference., 42005.

K. Mazur-Sniady, P. Sniady, y W. Zielichowski-Haber. Stochastic vibrations of beams with uncertain parameters subject to any random load. EURODYN 2005, C. Soize and G.I. Schuëller (eds)., millpress. rotterdam edition, 2005. ISBN 9059660331.

O. A. F. Mello. Helicopter flight simulation after rotor blade failure for accident investigation. (AIAA-98-0504), 1998.

MSC. MSC.Marc 2005, MSC.Dytran 2005 and 2008 and MSC.Patran 2005 and 2008. MacNealSchwendler Corporation, 2005. 
S. Nadarajah. Reliability for extreme value distributions. Mathematical and Computer Modelling, 37:915-922, 2003.

E. Nikolaidis, R. Hernández, y G. Maglaras. Evaluation of methods for reliability assessment when random variables are correlated. AIAA, 1312-CP, 1995.

N. Nolde. The Analysis of Extremes in Multivariate Models. PhD thesis, M.Sc. Statistics, Simon Fraser University, ETH ZURICH, 2010.

J. R. Olguin. 2015 accidents and incidents. Technical report, Instituto Boliviano de Historia Aeronáutica, Latin American Aviation Historical Society and Air-Britain (Historians) Ltd., Última actualización: 5 de enero de 2015, accedido el 12 abril 12 de 2015. URL http://www . aviacionboliviana.net/civil/accidentes/index.htm.

T. L. Paez. Random vibrations assessment of the state of the art. Technical Report 0469C, Experimental Structural Dynamics Department. Sandia National Laboratories, Albuquerque, New Mexico, 1999.

N.K. Parthasarathy. An efficient algorithm for blade loss simulations applied to a high-order rotor dynamics problem. PhD thesis, Texas A and M University, 2003.

P. Praks y J. Brozovsky. Simulation approaches for the efficient probabilistic reliability assessment of a concrete structure by the sbra method. Engineering mechanics, 17(5):299-305, 2010.

J. L. Pérez, L. H. Benítez, M. Oliver, y H. Climent. Survey of aircraft structural dynamics nonlinear problems and some recent solutions. The Aeronautical Journal, 115(1173), November 2011.

R. Rackwitz. Reliability analysis past, present and future. In 8th ASCE Speciality Conference on Probabilistic Mechanics and Structural Reliability., 2000.

C. Reményi y S. Staudacher. Systematic simulation based approach for the identification and implementation of as cheduling rule in the aircraft engine maintenance. International Journal of Production Economics, 2012. doi: 10.1016/j.ijpe.2012.10.022. URL http://dx.doi.org/ 10.1016/j.ijpe.2012.10.022.

M.O.W. Richardson y M.J. Wisheart. Case study: Analysis of the response of an aircraft structure caused by a propeller blade loss. Composites Part A, 27A:1123-1131, 1996. ISSN 1359-835X.

D. J. Rixen. A dual craig-bampton method for dynamic substructuring. Journal of Computational and Applied Mathematics, 168:383-391, 2004. 
J. B. Roberts y P.D. Spanos. Random Vibration and Statistical Linearization. John Wiley and Sons., 1999. ISBN 0-486-43240-8.

H. Rootzén y N. Tajvidi. Multivariate generalized pareto distributions. Bernoulli, 12(5):917-930, 2006.

K. Roscoe y T. Diermanse, F.and Vrouwenvelder. System reliability with correlated components: Accuracy of the equivalent planes method. Structural Safety, 57:53-64, 2015.

G.P. Sallee y D. M. Gibbons. Propulsion system malfunction plus inappropriate crew response $(\mathrm{psm}+\mathrm{icr})$. Technical report, AIA / AECMA, 1998.

A. Sarkar y R. Ghanem. Mid-frequency structural dynamics with parameter uncertainty. Seattle, WA, 42nd AIAA/ASME/ASCE/AHS/ASC Structures, Structural Dynamics, and Materials Conference and Exhibit., 42001.

Y. Shi, T. Swait, y C. Soutis. Modelling damage evolution in composite laminates subjected to low velocity impact. Composite Structures, 94:2902-2913, 2012.

M. Shinozuka y Y. K. Wen. Monte carlo solution of nonlinear vibrations. New York, New York, 9th AIAA Aerospace Science Meeting., 11972.

M. Shinozuka y J. N. Yang. Peak structural response to nonstationary random excitations. Anaheim, California, 4 1971. 12th AIAA/ASME/ASCE/AHS/ASC Structures, Structural Dynamics and Materials Conference.

S.K.. Sinha. Rotordynamic analysis of asymmetric turbofan rotor due to fan blade-loss event with contact-impact rub loads. Sound and Vibration, 332:2253-2283, 2013.

K. Sobczyk. Stochastic dynamics and reliability of degrading systems. Bulletin Of The Polish Academy Of Sciences. Technical Sciences., 54(1), 2006.

K. Sobczyk, S. Wedrychowicz, y B.F. Spencer. Dynamics of structural systems with spatial randomness. 33(11):1651-1669, 1996.

M.M. Sondhi. Random processes with specified spectral density and first order probability density. The Bell System Technical Journal, 62(3):679-701, 1983.

J. Song y A. Der Kiureghian. Joint first-passage probability and reliability of systems under stochastic excitation. Journal of Engineering Mechanics-ASCE, 132(1):65-77, 2006.

J.D. Sørensen. Notes in structural reliability theory and risk analysis. Technical report, Institute of Building Technology and Structural Engineering. Aalborg University, 2004. 
FSF Editorial Staff. In-flight separation of propeller blade results in uncontrolled descent and fatal accident to twin-turboprop commuter aircraft. Accident prevention, 54(2), 1997.

M.J. Stallone, V. Gallardo, A.F. Storace, L.J. Bach, G. Black, y E.F. Gaffney. Blade loss transient dynamic analysis of turbomachinery. Number AIAA-82-1052, Cleveland, Ohio, June 1982. AIAA/SAE/ASME. doi: 10.2514/6.1982-1057.

B. Sudret. Comparison of finite element reliability methods. Probabilistic Engineering Mechanics, 17:337-348, 2002.

R. Trandafir y S. Demetriu. Numerical simulation of non-gaussian random fields. Tehnical University of Civil Engineering of Bucharest, The 7th Balkan Conference on Operational Research, 2004.

B. Troclet, B. Hiverniau, M.N. Ichchou, L. Jezequel, K. Kayvantash, T. Bekkour, J.B. Mouillet, y A. Gallet. Fem/sea hybrid method for predicting mid and high frequency structure-borne transmission. The Open Acoustics Journal, 2:45-60, 2009.

M.J. Turner, R.W. Clough, H.C. Martin, y L.J. Topp. Stiffness and deflection analysis of complex structures. Journal of the aeronautical science, 23(9), 1956.

H.D. Unrau. Historic structure report: the south fork dam historical data. Technical report, Historic preservation Mid-Atlantic/North Atlantic team. National Park Service., Denver center branch, Colorado, 1979.

R. Vaicaitis, C. M. Jan, y M. Shinozuka. Nonlinear panel response and noise transmission from a turbulent boundary layer by a monte carlo approach. San Diego, California, 10th AIAA Aerospace Science Meeting., 11972.

P. S. Veers y S. Butterfield. Extreme load estimation for wind turbines - issues and opportunities for improved. AIAA Journal, 44, 2001.

F. Wang, Y. Sun, y H. Zeng. Study of uncontained turbine engine rotor failure airworthiness compliance verification method. Procedia Engineering, 17:531 - 541, 2011.

S. E. Webster y A. C. Bannister. Methods, applications and software for structural reliability assessment. Technical Report SL/WEM/R/M8663/5/01/C, Corus UK Limited. Swinden Technology Centre., 2001.

J.M.F. Wiggenraad y D.G. Zimcik. Frangibility of approach lighting structures at airport. Technical Report NLR-TP-2001-064, National Aerospace Laboratory, 2001.

J.M.F. Wiggenraad, A. de Boer, y R.H.W.M. Frijns. Impact simulation of a frangible approach light structure by an aircraft wing section. Technical Report NLR-TP-2000-618, National Aerospace Laboratory, 2000. 
J. Wijker. Mechanical Vibrations in Spacecraft Design. Springer-Verlag Berlin Heidelberg New York., 2004. ISBN 3-540-40530-5.

J. Wijker. Random Vibrations in Spacecraft Structures Design. Springer-Verlag Berlin Heidelberg New York., 2009. ISBN 978-90-481-2728-3.

P. H. Wirsching, T. L. Paez, y K. Ortiz. Random Vibrations. Theory and practice. John Wiley and Sons., dover edition, 2006. ISBN 0-486-45015-5.

P.A. Withey. Fatigue failure of the de havilland comet i. Engineering Failure Analysis, 4(2): 147-154, 1997. doi: 10.1016/S1350-6307(97)00005-8.

Y. T. Wu y Y. Shin. Probabilistic function evaluation system (profes) for maintenance optimization. Austin, Texas, 46th AIAA/ASME/ASCE/AHS/ASC Structures, Structural Dynamics and Materials Conference., 42005.

S. Xu y P.H. Chen. Prediction of low velocity impact damage in carbon/epoxy laminates. Procedia Engineering, 67:489-496, 2013.

H. Zhang y X. Li. Certification considerations for minimizing hazards caused by uncontained turbine engine and auxiliary power unit rotor failure. Procedia Engineering, 17:521 - 528, 2011.

Ma. Zheng-Dong y Qi. Chang. Multidisciplinary design optimization of elastomeric mounting systems in automotive vehicles. Number MI 48109-2121, Department of Mechanical Engineering, University of Michigan 1002 W.E. Lay Automotive Laboratory, 1231 Beal Avenue, Ann Arbor, August-September 2004.

H. S. Zibdeh y R. A. Heller. The use of the first passage method in service life prediction for rocket motors. HuntsviIle, Alabama, AI AAl ASM EISAEl ASEE 22nd Joint Propulsion Conference., 61986. 


\section{CONTRIBUCIONES ACADÉMICAS}

\section{A.1. Publicaciones propias de la tesis}

A continuación se muestran las publicaciones que han surgido directamente del trabajo aquí expuesto. La publicación [1] corresponde más o menos con los contenidos desarrollados en el capítulo 3. La publicación [2] corresponde con los contenidos desarrollados en el capítulo 4. Y finalmente la publicación [3] corresponde con los contenidos desarrollados en el capítulo 5.

1. I. Armendáriz, J. San Millán, J.M. Encinas, and J. Olarrea. Handbook of Materials Failure Analysis With Case Studies from the Aerospace and Automotive Industries. Chapter: Strategies for dynamic failure analysis on aerospace structures. Elsevier, 2015. ISBN: 9780-12-800950-5.

2. I. Armendáriz, J. López, J. Olarrea, M. Oliver, and H. Climent. Case study: Analysis of the response of an aircraft structure caused by a propeller blade loss. Engineering Failure Analysis, 37:12-28, 2014. ISSN 1350-6307. DOI: 10.1016/j.engfailanal.2013.11.0.

3. I. Armendáriz, J. Olarrea, and J. García-Martínez. Parametric analysis of a highly dynamical phenomena caused by a propeller blade loss. Engineering Failure Analysis, 57:528-543, 2015. ISSN 1350-6307. DOI: 10.106/j.engfailanal.2015.08.011.

\section{A.2. Publicaciones relacionadas con la tesis}

En estas publicaciones se plantean los análisis de distintos fenómenos: una estrategia de análisis estáticos de fenómenos que incluyen fallos [1] que es un complemento a la publicación [1] del apartado anterior, distintos análisis de crecimientos de grietas, delaminaciones y despegues en estructuras de material compuesto [2] y [3] en donde se utilizan herramientas avanzadas para su análisis y, finalmente, se realiza un diseño y análisis preliminar de las estructuras soporte de un telescopio para observar exoplanetas que tiene que soportar cargas tan fuertes como son las del lanzamiento y a la vez mantener una gran precisión para realizar las observaciones [4] y [5].

1. I. Armendáriz, J. San Millán, J. García-Martínez, and R. González. Handbook of Materials Failure Analysis With Case Studies from the Aerospace and Automotive Industries. 
Chapter: Strategies for static failure analysis on aerospace structures. Elsevier, 2015. ISBN: 978-0-12-800950-5.

2. J. San Millán and I. Armendáriz. Damage Growth in Aerospace Composites. Chapter: Delamination and debonding growth in Composite structures. Springer, 2015. ISBN 9783-319-04003-5.

3. I. Armendáriz, R. González, J. San Millán, and J. García-Martínez. Delamination growth in damage tolerance composite structures. Sorrento, Italy, 9th International Conference on Composite Science and Technology, 2013. ISBN 978-1-60595-113-3.

4. G. Ramos-Zapata, T. Belenguer, A. Balado, J. Barandiarán, I. Armendáriz, M. Reina, C. Arza, S. Rodríguez, M.A. Alcacera, J.A. Fernández, E. Muñoz, G. Tinetti, P. Eccleston, B. Swinyard, M. Ferlet, I. Bryson, and M.R. Zapatero. Echo SWIR: Exoplanet atmospheres characterization observatory sort-wave infrared channel of the echo payload. 2012. ISSN 0277-786X. DOI: 10.1117/12.925957.

5. G. Ramos-Zapata, T. Belenguer, A. Balado, J.M. Encinas, I. Armendáriz, A. Carretero, C. Arza, M.A. Alcacera, J.A. Fernández, and E. Muñoz. Exoplanet atmospheres characterization obervatory payload short-wave infrared channel: Echo swir. 2014. ISSN 0277-786X. DOI: $10.1117 / 12.2054876$. 


\section{MEDIOS Y PROGRAMAS DE SOFTWARE}

\section{B.1. Medios para la realización de la tesis}

Los medios utilizados para la realización del trabajo han sido monografías, artículos disponibles tanto en los departamentos como en las bibliotecas de INTA y de la UPM. En cuanto al software de simulación utilizado ha sido MATLAB (licencias propiedad del INTA 2010-2015 y desde agosto-2015 de la UPM), Latex (licencias para WinEdt 6.0 propiedad del Departamento de Matemática Aplicada y Estadística). Y en lo que respecta al software de cálculo estructural comercial se ha utilizado MSC.Patran, MSC.Marc, MSC.Dytran y MSC.Nastran, y en general el software de Mac Neal-Schwendler Corporation (licencias propiedad del INTA). El equipo e infraestructuras que se han dispuesto han sido las disponibles en el Departamento de Materiales y Estructuras y del Departamento de Matemática Aplicada y Estadística.

\section{B.2. Flujo de integración en el programa Matlab}

En esta sección se describe el esquema que se ha seguido para la resolución del problema planteado. El flujo del programa es el mostrado en la figura B.1. Para simular el fenómeno se lanza el programa principal que, según sea las opciones elegidas, simula el grupo de caso de carga seleccionado. Primero se plantean una serie de datos y parámetros iniciales para pasar al bucle principal de integración. Una vez se haya simulado y reproducido el fenómeno se sale del bucle y se almacenan los datos de salida.

\section{B.2.1. Datos y parámetros iniciales o constantes}

Inicialmente hay una serie de indicadores secundarios para la elección de las opciones de resultados (pintar gráficas, escribir en hoja de cálculo los resultados,... ). Luego se seleccionan parámetros importantes como, por ejemplo, lanzar el modelo completo o sólo el motor, el tipo de método de integración ( $\beta$-Newmark, Runge-Kutta de orden 2, Runge-Kutta de orden 3 ó Runge-Kutta de orden 4),... A continuación se eligen los datos de inicio de la integración: el tiempo inicial, el tiempo final, el número de pasos, el número de simulaciones a realizar. Se elige 


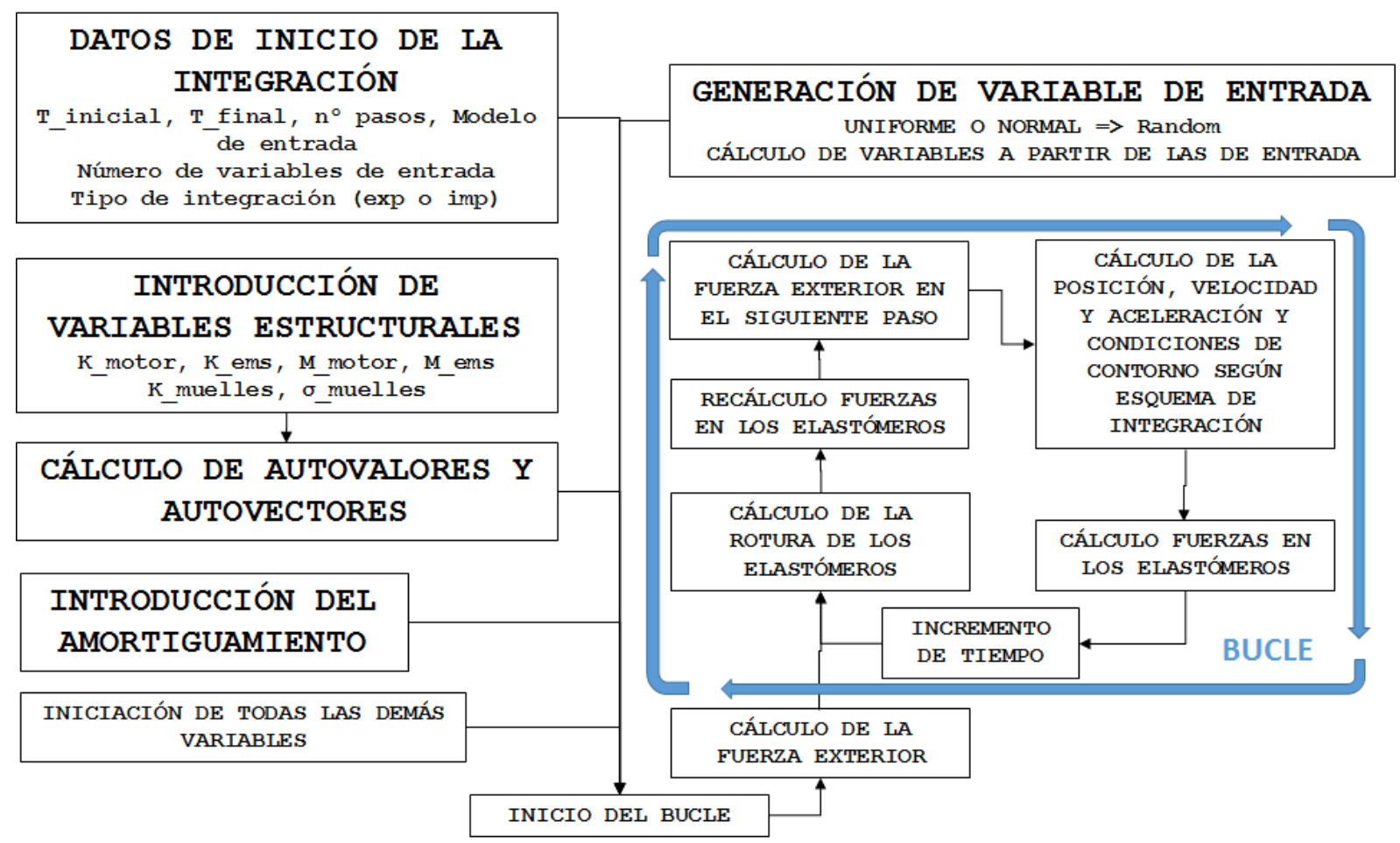

Figura B.1: Flujo seguido por el programa

que grupo de casos de carga se quiere lanzar de entre las cinco variables de entrada posible, se podría lanzar cualquier combinación de ellas pero se han pre-configurado las indicadas en el apartado 5.3. Seguidamente se obtienen la relación de valores de las variables de entrada según sean el número de simulaciones y el grupo de casos de carga a simular. Después, se realiza la inicialización de la mayoría de las variables a utilizar. Según sea el método de integración se inicializarán unas u otras variables. Se introducen los puntos de comportamiento de la fuerza de los elastómeros en función del desplazamiento y también de las matrices de masa y de rigidez del motor, y de la bancada si está elegido el modelo completo. Todos ellos se introducen llamando a subrutinas que contienen los valores.

A modo informativo y para chequear la idoneidad del paso de tiempo empleado se calculan los modos propios del sistema mediante otra subrutina, y se compara con el paso de tiempo para ver si es mayor que el paso de tiempo crítico. Si no lo es se indica mediante un mensaje.

Finalmente se introduce el amortiguamiento estructural según lo indicado en el apartado 5.2.3.3 y se preparan las matrices para el bucle principal.

\section{B.2.2. Bucle principal}

Se realizan las consideraciones oportunas, según sea el método de integración, para el caso del primer paso en el bucle. Después, se pasa al bucle más general y se llama a la subrutina del cálculo de las fuerzas en los elastómeros. A continuación se llama a la subrutina del cálculo de si 
hay rotura de alguno de los elastómeros. Luego se llama a la subrutina del cálculo de las fuerzas exteriores (las fuerzas debidas a la pérdida de la pala). Y ya una vez calculadas estas fuerzas y según se hayan roto o no los muelles se estiman las cargas definitivas en los muelles.

Entonces, se llega la integración del paso que depende totalmente del método de integración elegido. Se calculan además muchas otras variables como, por ejemplo, las relacionadas con la energía.

Finalmente se guardan las variables que se necesiten en el siguiente paso de integración y se incrementa el tiempo.

\section{B.2.3. Datos de salida}

Esta parte del código se ha restringido a las partes más representativas ya que durante la investigación han existido innumerables formas de tratar los datos de salida. A modo de resumen se puede decir que tras la integración se calculan datos máximos y mínimos de ciertas variables, se ordenan las variables de salida para ser representadas y se identifican las secuencias de fallo.

Finalmente se escriben, si ha sido requerido, los resultados según se hayan declarado las variables en la parte B.2.1 y se guardan, si ha sido requerido, las variables en un fichero. 